\title{
Dissertation
}

\section{Thermal Transport in Strongly Correlated Rare-Earth Intermetallic Compounds}

zur Erlangung des akademischen Grades

Doctor rerum naturalium (Dr. rer. nat.)

vorgelegt der Fakultät Mathematik und

Naturwissenschaften der Technischen Universität Dresden

von Diplom-Physikerin

\section{Heike Pfau}

geboren am 24. Juni 1984 in Frankfurt (Oder).

Max-Planck-Institut für Chemische Physik fester Stoffe

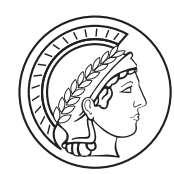




\section{Gutachter}

Prof. Dr. Frank Steglich

Prof. Dr. Joachim Wosnitza

Prof. Dr. Cornelius Krellner

Beginn der Promotion am 01.10.2010

Eingereicht am 16.12.2014

Verteidigt am 30.03.2015 


\section{Contents}

\begin{tabular}{lll}
\hline List of Symbols & iii
\end{tabular}

List of Abbreviations $\quad$ v

$\begin{array}{llr}1 \text { Introduction } & 1\end{array}$

2 Theoretical Models

2.1 Fermi Liquid . . . . . . . . . . . . . . . . . . . . . 5

2.2 The Kondo Effect in $4 f$ Electron Systems $\ldots \ldots \ldots$

$2.2 .1 \quad$ Single-Ion Kondo Effect $\ldots \ldots \ldots$. . . . . . . . . . . . . . . . . 7

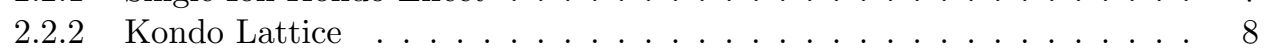

$2.2 .3 \quad$ RKKY Interaction and Doniach Diagram . . . . . . . . . . . . 9

2.3 Classical Phase Transitions $\ldots \ldots \ldots$. . . . . . . . . . . . . . . . . . . 10

2.4 Quantum Phase Transitions . . . . . . . . . . . . . . . . . . . . . . . . . . 11

2.5 Topological Transitions $\ldots \ldots \ldots \ldots \ldots \ldots$

2.6 Superconductivity $\ldots \ldots \ldots \ldots \ldots \ldots \ldots$

$2.6 .1 \quad$ Properties of Conventional Superconductors . . . . . . . . . . . . . . 17

2.6 .2 Properties of Unconventional Superconductors . . . . . . . . . . . . 19

$2.6 .3 \quad$ Multiband Superconductivity $\ldots \ldots \ldots$

2.7 Transport in Solids . . . . . . . . . . . . . . . . . . . . . . . . . . 19

$2.7 .1 \quad$ Boltzmann Equation . . . . . . . . . . . . . . . . . . . . . . . 20

2.7 .2 Thermal Conductivity . . . . . . . . . . . . . . . . . . . . . . . 21

2.7 .3 Wiedemann-Franz Law $\ldots \ldots \ldots \ldots \ldots \ldots$

2.7 .4 Thermopower . . . . . . . . . . . . . . . . . . . . . . . . . . 24

\begin{tabular}{|lll}
3 & Experimental Techniques for Transport Measurements & 27
\end{tabular}

3.1 General Principle of Thermal Transport in a Dilution Refrigerator . . . . . . 27

3.2 The Three Set-ups Used $\ldots \ldots \ldots \ldots . \ldots \ldots$

3.3 Measurement Procedure - Steady State . . . . . . . . . . . . . . . . . . . . . 30

3.4 Measurement Procedure-Pseudo AC . . . . . . . . . . . . . . . . . . . . . 31

3.5 Origin and Estimation of Measurement Uncertainties . . . . . . . . . . . . 33

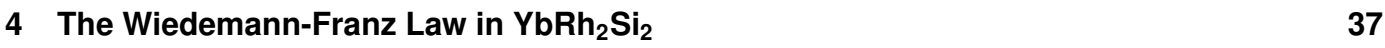

4.1 Quantum Criticality in $\mathrm{YbRh}_{2} \mathrm{Si}_{2}$-Phenomenology . . . . . . . . . . . 37

4.2 Quantum Criticality in $\mathrm{YbRh}_{2} \mathrm{Si}_{2}$-Theoretical Concepts . . . . . . . . . . . 39

4.2 .1 The Kondo-Breakdown Scenario . . . . . . . . . . . . . . . . . . 40

4.2 .2 The Lifshitz Scenario . . . . . . . . . . . . . . . . . . . . . . . . . . . 41

$4.2 .3 \quad$ Critical Quasiparticle Theory . . . . . . . . . . . . . . . . . 41

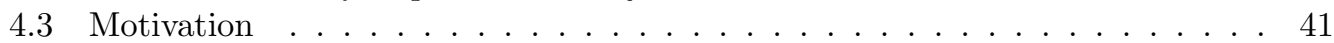

4.4 Experimental Methods $\ldots \ldots \ldots \ldots$. . . . . . . . . . . . . . . . . . . 42 
4.5 Thermal and Electrical Transport . . . . . . . . . . . . . . . . . . . . 43

4.5 .1 Phonon Contribution . . . . . . . . . . . . . . . . . . . 43

4.5 .2 Low-Temperature Data and the Wiedemann-Franz Law . . . . . . . . 44

4.5.3 Comparison with Results from Other Groups . . . . . . . . . . . . . . 49

4.6 Conclusion $\ldots \ldots \ldots \ldots \ldots \ldots \ldots \ldots$

5 Kondo Lattices in Magnetic Field

5.1 Introduction and Motivation . . . . . . . . . . . . . . . . . . . 53

5.2 Experimental Methods . . . . . . . . . . . . . . . . . . . . . 54

$5.3 \mathrm{CeRu}_{2} \mathrm{Si}_{2} \ldots \ldots \ldots \ldots \ldots \ldots \ldots \ldots$

5.3 .1 The Metamagnetic Transition . . . . . . . . . . . . . . . . . . . 55

5.3 .2 Transport Data . . . . . . . . . . . . . . . . . . . . 56

$5.4 \mathrm{YbRh}_{2} \mathrm{Si}_{2} \ldots \ldots \ldots \ldots \ldots \ldots$

5.4 .1 The High-Field Transition . . . . . . . . . . . . . . . . . . . . . . 58

5.4 .2 Transport Data . . . . . . . . . . . . . . . . . . 59

5.4 .3 Renormalized Band Structure Calculations for $\mathrm{YbRh}_{2} \mathrm{Si}_{2}$. . . . . . . 59

5.5 Rigid-band-shift Models for the Field-induced Transitions in $\mathrm{CeRu}_{2} \mathrm{Si}_{2}$ and

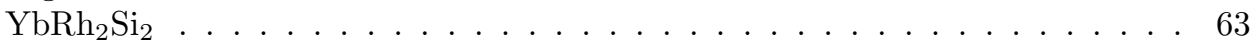

5.5 .1 Transport Signatures . . . . . . . . . . . . . . . . . . 64

5.5 .2 Thermodynamic Signatures . . . . . . . . . . . . . . . . . 66

5.6 The Mott Formula in $\mathrm{CeRu}_{2} \mathrm{Si}_{2}$ and $\mathrm{YbRh}_{2} \mathrm{Si}_{2} \ldots \ldots \ldots$. . . . . . . . . . 66

5.6 .1 General Approach . . . . . . . . . . . . . . . . . . . 67

5.6 .2 Phonon Drag . . . . . . . . . . . . . . . . . . . 68

$5.6 .3 \quad$ Validity in $\mathrm{YbRh}_{2} \mathrm{Si}_{2}$ but not in $\mathrm{CeRu}_{2} \mathrm{Si}_{2} \ldots \ldots \ldots$. . . . . . . . . 68

5.6.4 Comparison of Thermopower and Density of States in $\mathrm{YbRh}_{2} \mathrm{Si}_{2}$. . . 71

5.6 .5 The $q$ Ratio . . . . . . . . . . . . . . . . . . . . . . . . . . . . . . 72

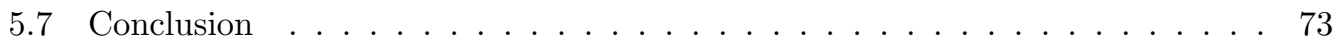

6 The Superconducting Order Parameter of $\mathrm{LaPt}_{4} \mathrm{Ge}_{12}$

6.1 Introduction and Motivation . . . . . . . . . . . . . . . . 75

6.2 Experimental Methods . . . . . . . . . . . . . . . . . . . . . . 77

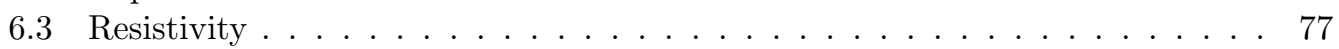

6.4 Specific Heat . . . . . . . . . . . . . . . . . . . . . . . . . . . . . . . . . . . . . . . . . . . .

6.5 Thermal Conductivity . . . . . . . . . . . . . . . . . . . 79

6.5.1 T Dependence of the Thermal Conductivity . . . . . . . . . . . . . 79

6.5.2 H Dependence of the Thermal Conductivity . . . . . . . . . . . . . . . 81

6.5.3 Field-angle Dependence of the Thermal Conductivity . . . . . . . . . 86

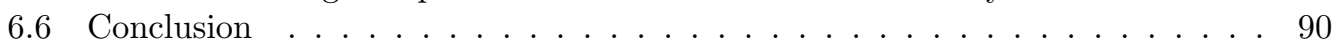

$\begin{array}{lll}7 & \text { Summary and Outlook } & 91\end{array}$

\begin{tabular}{ll}
\hline Bibliography & 95
\end{tabular} 


\section{List of Symbols}

The following list specifies symbols used throughout the text. Very specific ones used only in isolated passages have been omitted. In cases, where it is necessary, we use bold symbols to indicate vectors.

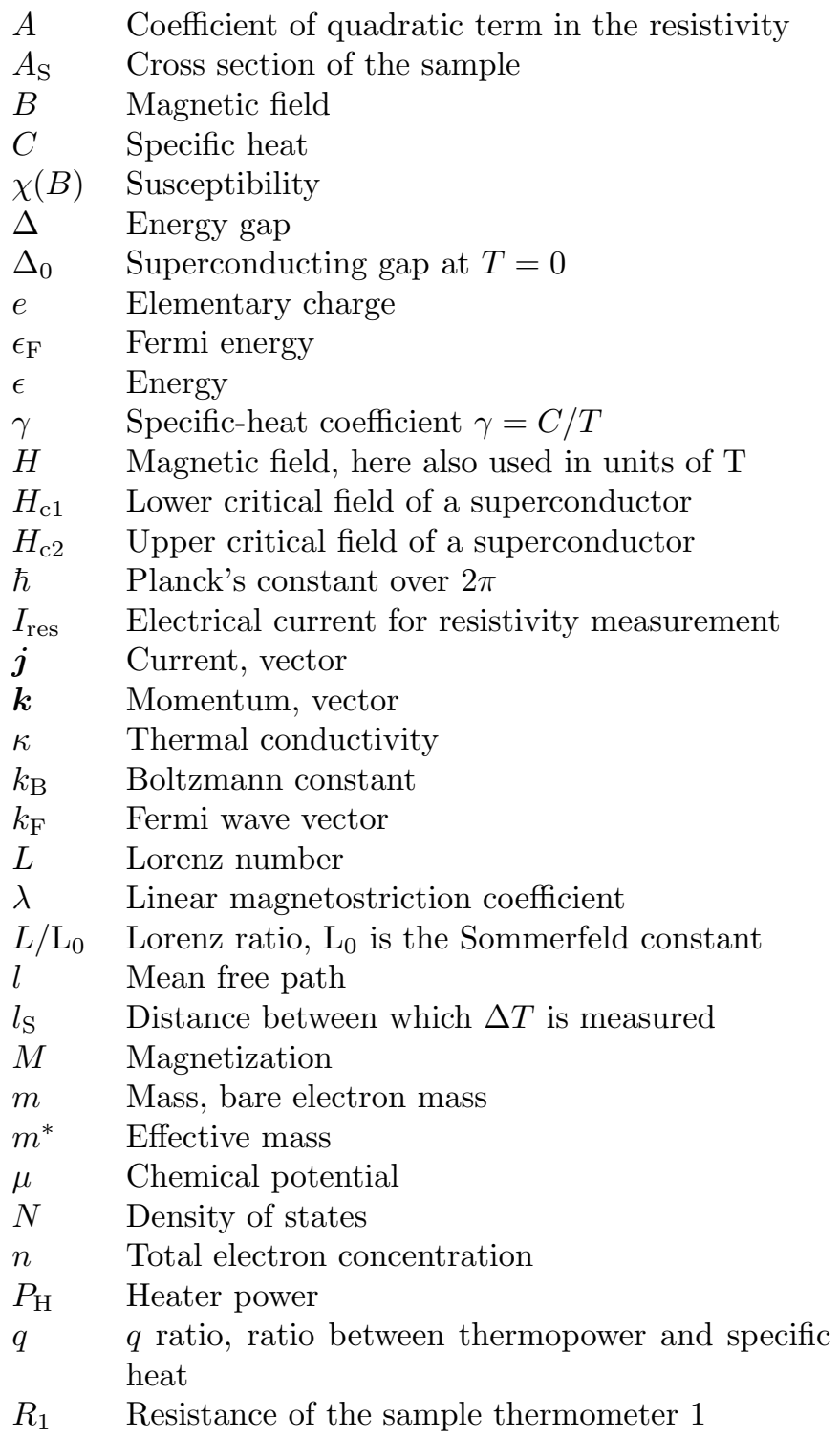


$R_{2} \quad$ Resistance of the sample thermometer 2

$\rho \quad$ Electrical resistivity

$\rho_{0} \quad$ Residual resistivity

$S$ Thermopower

$\sigma \quad$ Electrical conductivity

$T$ Temperature

$\tau \quad$ Scattering time

$\Delta T \quad$ Temperature gradient

$\Theta \quad$ Debye temperature

$T_{\mathrm{K}} \quad$ Kondo temperature

$T_{\mathrm{MC}} \quad$ Temperature of the mixing chamber or cold finger

$U_{\text {res }} \quad$ Voltage used to determine resistivity

$U_{\mathrm{T}} \quad$ Thermovoltage

$v \quad$ Velocity

$v_{\mathrm{F}} \quad$ Fermi velocity 


\title{
List of Abbreviations
}

\author{
AF antiferromagnetic \\ BCS Bardeen-Cooper-Schrieffer \\ BRT Bardeen, Rickayzen, and Tewordt \\ dHvA de Haas-van Alphen \\ DOS density of states \\ F "Franz" \\ FFT fast Fourier transformation \\ FM ferromagnetic \\ MMT metamagnetic transition \\ NMR nuclear magnetic resonance \\ PPMS Physical Property Measurement System \\ QCP quantum critical point \\ R "Rotator" \\ RB renormalized band \\ RKKY Ruderman-Kittel-Kasuya-Yosida \\ RRR residual resistivity ratio \\ S "SHE" \\ SDW spin-density wave \\ $\mu \mathrm{SR} \quad$ muon spin rotation \\ WF Wiedemann-Franz
}





\section{Introduction}

Richard Feynman aptly summarized the key principle of natural sciences in his famous lectures Fey63: "The test of all knowledge is experiment." It has been a guidance for all physicists, who try to extract the fundamental laws of our world from more and more complicated experimental arrangements. This is also true for condensed-matter physicists, who investigate macroscopic objects, which are composed of a large number of elementary particles. These objects cannot be understood only in terms of a simple extrapolation of the properties of the single constituents. Instead, the complex arrangement of elementary particles causes new phenomena to emerge. Any small crystal in our salt shaker consists of a myriad of particles, every of them obeys the fundamental laws of quantum electrodynamics. The behaviour of the crystal as a whole with its beautiful colourless rectangular shape, however, is governed by additional mechanisms. To understand the fundamental concepts of such emergence is the subject of condensed-matter physics. This field, compared to other branches of physics, has the advantage, that we can actually see and touch our objects of study in most cases. We can carry them into our laboratories and directly measure their properties. Therefore, experiment and theory progress in parallel, which leads to a mutually inspiring scientific environment in this research field.

In this dissertation, we will investigate metallic materials. Modern condensed-matter physics of metals rests on two cornerstones, which form the theoretical basis of our research. The first one is the concept of the Fermi liquid. It was introduced by Lev Landau in 1956. In this effective single-particle model, we can treat the electrons in a metal independently as if each of them moves in a background, which is generated by all other electrons. The electrons confined to a solid - called electronic quasiparticles - share many properties with free electrons: they have the same charge, spin, and momentum. But other properties such as the mass of these quasiparticles are renormalized. In some materials the difference can be as large as a factor of one thousand.

Metals can also exist in the form of different phases: They may be in a magnetic state (e.g. ferromagnetic or paramagnetic); they may form a crystallographic structure (e.g. cubic or hexagonal); or they may have different aggregation states (e.g. fluid or solid). We can understand these phases and transitions between them with the help of the second cornerstone - Landau's concept of phase transitions. It was developed by Lev Landau in the 1930s and further advanced by Vitaly Ginzburg and Kenneth Wilson in the 1960s and 1970s. According to this model, every phase is connected to a set of certain symmetries. A transition from one phase to another signals a breaking of one of these symmetries. For instance when water freezes into an ice crystal, the translational symmetry breaks: The beautiful symmetric phase of the crystal is - counter-intuitively-less symmetric than the perfect homogeneity of a fluid.

These two theories - the Fermi-liquid theory and the concept of phase transitions - form 
the starting point of our research. A major aim of current condensed-matter physics is to find and investigate systems, which deviate from these established models. From the discovery of novel phenomena and the failure of established pictures, new physical concepts can emerge, which broadens our horizon of knowledge and comprehension. Strongly correlated materials are a wonderful playground for this search, because the strong interactions between the electrons often require a description beyond single-particle pictures. The electrons in these compounds do not behave independently but like a fish in a shoal.

The work presented in this thesis is also driven by the search for cases where fundamental physical laws are violated. We will study three different questions where previous investigations already indicated unconventional behaviour. To understand this behaviour more deeply, we will investigate the validity or violation of specific fundamental physical laws using transport, in particular low-temperature thermal-transport measurements.

Transport is an established measurement technique, which gives access to many important quantities describing the particles in a solid, for instance about the number, the velocity, and the charge of particles, and their interaction with other particles. But transport is also one of the most difficult properties to describe theoretically, because it occurs only when the system is out of equilibrium. From a simple classical viewpoint transport shares many properties with a pinball machine: In such a machine, the pinballs move along the gradient of potential energy from the top to the bottom. On their way, they collide with little posts, the walls and other pinballs in the machine. One can classify machines by their conductivity, which simply compares the inclination with the average time it takes a pinball to move from the top to the bottom of a machine. Inexperienced players will of course favour a machine with a low conductivity.

Transferred to a solid, the pinballs represent particles such as electronic quasiparticles or phonons. The latter are the quantum-mechanical particles describing vibrations of the crystal lattice. These particles can collide with defects, the sample boundary or with other particles. Their motion is driven by thermal or electrical gradients and we will classify materials by their electrical conductivity, their thermal conductivity, or their thermopower in this work.

It is often challenging to extract the information about the particles provided by transport measurements out of the experimental data, because several species of particles can contribute to the transport and they can scatter on even more types of particles and defects. In addition, the classical approach of the pinball machine to transport remains insufficient. The particles and their interactions are highly quantum mechanical in nature. These complex interactions in turn are responsible for the unconventional phenomena we want to study in strongly correlated materials. Due to these challenges, transport and in particular thermal-transport measurements, are used to answer specific physical questions in systems, which have been characterized extensively before. However, the lack of theoretical background for many aspects of transport properties also indicates that there is great potential to develop such descriptions on the basis of new, high-precision measurements.

In this respect, transport measurements at low temperatures have several advantages. The number of some sorts of particles, particularly phonons, is suppressed at low temperatures and we can study solely electronic quasiparticles or other low-energy excitations. Due to the small number of phonons, we can also neglect them as scattering centres. In Chapter 3 we will describe the technique used to measure thermal transport at low temperatures. Chapter 2 will introduce the theoretical background of transport together with the most important theoretical concepts used throughout this dissertation. These chapters are followed by the 
three transport investigations performed in the framework of this thesis.

In our first study (Chapter 4), we will investigate a magnetic phase transition. We can change its transition temperature by an external parameter, just as we can decrease the melting temperature of ice when we apply pressure by our ice skaters. When the transition temperature is suppressed to zero, we call this a quantum phase transition. The properties of materials around such a transition differ drastically from the Fermi-liquid model. We will investigate the material $\mathrm{YbRh}_{2} \mathrm{Si}_{2}$, the magnetic order of which can be suppressed by a small external magnetic field. The properties of the quantum phase transition in $\mathrm{YbRh}_{2} \mathrm{Si}_{2}$ are very unconventional and cannot be described within Landau's theory of phase transitions.

To investigate the unconventional properties of $\mathrm{YbRh}_{2} \mathrm{Si}_{2}$, we study the Wiedemann-Franz law, which is named after Gustav Heinrich Wiedemann and Rudolph Franz. They discovered in 1853 that the ratio of the thermal and the electrical conductivity takes the same value in almost all metals. Ludwig Lorenz realized in 1872 that this ratio is linearly dependent on temperature. The constant of proportionality takes a universal value. Later on, those empirical results were theoretically verified for a large number of physical systems. To date, the Wiedemann-Franz law is one of the most fundamental laws in condensed-matter physics, which is obeyed in virtually all metals. We want to study if the special properties connected to the non-Fermi liquid in $\mathrm{YbRh}_{2} \mathrm{Si}_{2}$ also influence the validity of the Wiedemann-Franz law. A violation has dramatic physical consequences, as the established picture of electronic quasiparticles would no longer be applicable.

In the second study (Chapter 5), we investigate topological transitions. If two objects have the same topology, the shape of one can be deformed continuously into the shape of the other. The most prominent example is the shapes of a coffee mug and a doughnut, which have the same topology. Topological transitions are beyond the concepts of Landaus theory of phase transitions. Topological changes of the Fermi surface of a metal are called Lifshitz transitions. We will study Lifshitz transitions in the two materials $\mathrm{YbRh}_{2} \mathrm{Si}_{2}$ and $\mathrm{CeRu}_{2} \mathrm{Si}_{2}$. To uncover the systematics of these transitions and explain the magnetic-field evolution of the two compounds, we will investigate another fundamental physical law- the Mott formula. This law is named after Sir Nevill Francis Mott. It relates the electrical conductivity and the thermopower and is, therefore, a transport phenomenon such as the Wiedemann-Franz law. The ratio is proportional to temperature and the prefactor is a universal number. We will use the validity and the violation of the Mott formula to analyse the behaviour of $\mathrm{CeRu}_{2} \mathrm{Si}_{2}$ and $\mathrm{YbRh}_{2} \mathrm{Si}_{2}$ in magnetic fields.

In the third project (Chapter 6), we will investigate a superconducting material. Superconductors have the ability to conduct electricity without loss and to expell magnetic fields. They are a wonderful example for the beauty and surprise of emergence in condensed-matter physics. The transition into the superconducting state is in general a phase transition in the sense of Landau. Bardeen, Cooper, and Schrieffer developed a microscopic theory for superconductivity in 1957 called BCS theory, which could successfully describe many superconductors. However, there are other materials whose superconducting state cannot be understood within the conventional BCS picture. The first one, $\mathrm{CeCu}_{2} \mathrm{Si}_{2}$, was discovered by Frank Steglich in 1978 and it was followed by many others such as the high-temperature cuprate superconductors in the 1980s. In our study, we will use thermal-conductivity measurements to investigate if the BCS theory can be applied to the superconductor $\mathrm{LaPt}_{4} \mathrm{Ge}_{12}$ or if this material also belongs to the class of unconventional superconductors.

Although our three projects are thematically quite different, they all illustrate that new physical knowledge stems from the failure of established theories during experimental tests. 
In this respect, thermal transport experiments are a challenge and a great opportunity at the same time due to the important information they provide and the complexity of their interpretation. 


\section{$2^{\text {conem }}$ 2 Theoretical Models}

The following chapter will introduce the important concepts and formulas needed throughout the thesis and is based on standard literature. It starts from the fundamental Fermi-liquid theory, which is presented following Refs. Ash76, Enn05. It then introduces Kondo physics Hew93. Col02, classical and quantum phase transitions Ash76, Voj03, topological transitions Bla94, Var89, Kos04 and superconductivity Min99 Ann10, Buc04. The last part is devoted to transport in general and thermal transport in particular Zim60.

\subsection{Fermi Liquid}

The challenge of condensed-matter physics is the treatment of the enormous number of $10^{23}$ interacting particles inside a solid. Since it is impossible to calculate the wave function of all those particles, physicists handle them e.g. by statistical methods. Drude proposed such a theory, which describes the electrons in a metal as a gas of non-interacting classical particles. Sommerfeld formulated the quantum-mechanical version of the Drude theory and treated the electrons as fermions. This model became known as the Fermi gas. Despite its simplicity, it describes very successfully many properties of metals such as a well-defined Fermi surface, a linear-in- $T$ specific heat, a temperature-independent paramagnetic susceptibility and the Wiedemann-Franz law.

Deviations from the Fermi-gas model were, however, detected in systems with strong interactions, first in liquid $\mathrm{He}^{3}$ and later also in metallic solids. These discrepancies were treated by Landau in an extension to the Fermi gas model - the Fermi liquid. The ground state of the Fermi liquid can be adiabatically connected to the ground state of the Fermi gas while gradually turning on the interactions between the electrons. Landau introduced the notion of quasiparticles, which are the elementary excitations of the Fermi liquid and which have a one-to-one correspondence to the excitations of the Fermi gas, although now with a finite lifetime. Thus, quasiparticles represent a renormalized version of their non-interacting counter parts. The renormalization is accounted for by Landau parameters $F_{l}^{s}, F_{l}^{a}$ and becomes most manifest in the mass enhancement,

$$
m^{*}=m\left(1+\frac{F_{1}^{s}}{3}\right),
$$

were $m^{*}$ is called the effective mass of the qusiparticles and can be as large as 1000 times the bare electron mass $m$, for example in heavy-fermion systems. The mass renormalization is accompanied by a large density of states (DOS) at the Fermi energy $\epsilon_{\mathrm{F}}$,

$$
N\left(\epsilon_{\mathrm{F}}\right)=\frac{m^{*} k_{\mathrm{F}}}{\pi^{2} \hbar^{2}},
$$




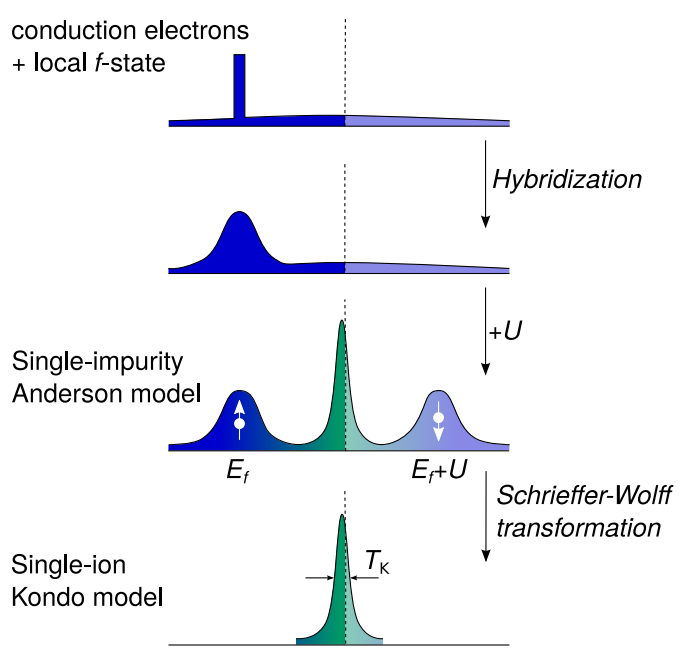

Figure 2.1: Ingredients for the Kondo model. (top to bottom) A magnetic impurity added into a sea of conduction electrons forms a resonance due to hybridization. Gradually turning on the Coulomb interaction $U$ splits this resonance, which gives rise to two peaks in the spectral function at $E_{f}$ and $E_{f}+U$ with opposite spins. They lead to a formation of local moments. Fluctuations between the spin-up and spin-down state create a third peak in between, which is called "Kondo resonance". The hybridization and the Coulomb interaction are the key ingredients for the single-impurity Anderson model. The Schrieffer-Wolff transformation integrates out the higher-energy physics of this model. The "Kondo resonance" remains and can be described within the single-ion Kondo model.

using the Fermi vector $k_{\mathrm{F}}$ and Planck's constant $\hbar$. The temperature dependences of the specific heat and the susceptibility stay the same compared to the Fermi gas, however, they are also renormalized. The specific heat $C, e . g$., can now be written in terms of the enhanced DOS as

$$
\frac{C}{T}=\frac{\pi^{2}}{3} k_{\mathrm{B}}^{2} N\left(\epsilon_{\mathrm{F}}\right)
$$

where $k_{\mathrm{B}}$ is the Boltzmann constant. A very characteristic property of the Fermi liquid is the resistivity $\rho$. It contains now a $T^{2}$ term due to the electron-electron scattering,

$$
\rho=\rho_{0}+A T^{2}
$$

$\rho_{0}$ represents the residual resistivity from defect scattering. Another remarkable result of the Fermi liquid theory can be extracted from the ratio of the $A$ coefficient and of the linear specific-heat term $\gamma=C / T$. The so-called "Kadowaki-Woods ratio",

$$
R_{\mathrm{KW}}=\frac{A}{\gamma^{2}}
$$

is found to be constant in certain groups of materials. In heavy-fermion systems the value of $R_{\mathrm{KW}}$ is approximately $10 \mu \Omega \mathrm{cm}(\mathrm{molK} / \mathrm{J})^{2}$, whereas it is 25 times smaller in $d$-electron systems.

\subsection{The Kondo Effect in $4 f$ Electron Systems}

In a simple metal, the electrical conductivity is usually determined by three scattering mechanisms: Scattering of electrons on defects leads to a constant resistivity at low temperaturesthe residual resistivity. Scattering on phonons and Fermi-liquid-like electron-electron scattering leads to a monotonic increase of the resistivity with increasing temperature. In contrast to this picture, de Haas et al. found a minimum in the resistivity of gold in 1934 


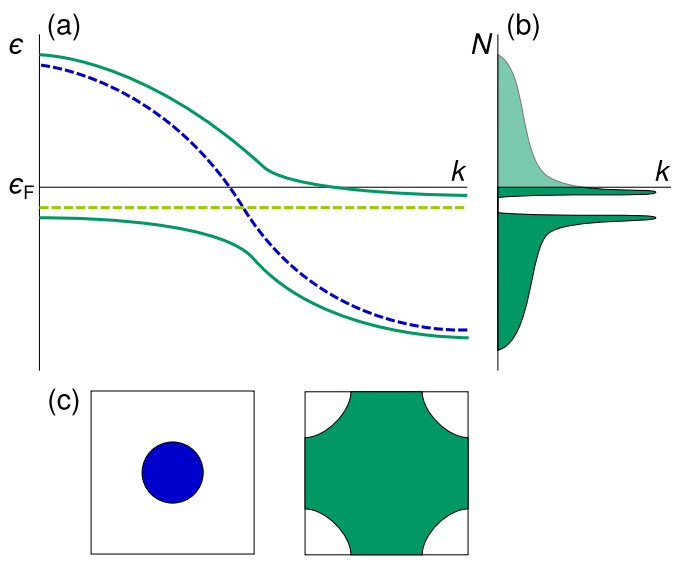

Figure 2.2: Bands in the Kondo-lattice model. (a) The injection of a composite quasiparticle state (light green) at each site and its hybridization with the conduction electrons (blue) leads to the formation of heavy bands (dark green) with a hybridization gap in between. (b) This gap separates two sharp peaks in the DOS for these heavy bands. (c) Due to the hybridization, the Fermi surface changes from a small one, which only counts the conduction electrons (left) towards a large one, which also contains the composite quasiparticles (right). After Col02.

[Haa34] indicating that there must be an additional scattering mechanism, which increases its strength as the temperature is lowered. Later on, the improvement of the crystal-growth techniques permitted the control of the impurity concentration in metals and a connection between magnetic impurities and the resistance minimum could be established [Ber64]. This discovery paved the way to resolve the long-standing puzzle of the resistance minimum. The mechanism is related to a scattering process of conduction electrons off magnetic impurities involving a spin flip and is called the Kondo effect.

\subsubsection{Single-Ion Kondo Effect}

To understand the Kondo effect we start with the impurity Anderson model. It describes localized electrons, e.g. $4 f$ electrons, in a sea of conduction electrons (Figure 2.1). The key element is a competition between two types of interactions: First, the hybridization between $f$ and conduction electrons and second, the repulsive Coulomb interaction between the electrons of a doubly occupied $f$ state. While the first leads to a formation of a resonance, the second tends to split this resonance into a spin-up and spin-down component and consequently local moments form. Importantly, a finite probability for the spin to tunnel between the up and down configuration leads to spin fluctuations of the local moment and to a resonance between the two spin states. This resonance is a manifestation of the Kondo effect and is called "Kondo resonance".

The interaction $U$ which drives the local-moment formation is of the order of $10 \mathrm{eV}$. On the other hand, the energy range of the Kondo physics is of the order of $1 \mathrm{meV}$. Therefore, it is possible to extract the physics of the Kondo effect by a renormalization approach, i.e., the high-energy physics is integrated out step by step. In our case, the so-called Schrieffer-Wolff transformation reduces the Anderson impurity model to the single-impurity Kondo model, in which only the impurity spin $\boldsymbol{S}$ is left and couples to the conduction electrons via an exchange interaction $J$ :

$$
\mathcal{H}=\sum_{\boldsymbol{k}, \sigma} \epsilon_{\boldsymbol{k}} c_{\boldsymbol{k} \sigma}^{\dagger} c_{\boldsymbol{k} \sigma}+J \boldsymbol{S} \cdot \boldsymbol{s}
$$

where $s$ is the spin density of the conduction electrons at the impurity site. The first attempt to solve this problem was made by Kondo within a perturbation approach [Kon64]. He found for an antiferromagnetic coupling $J$ a $\log T$ term in the resistivity, which could 


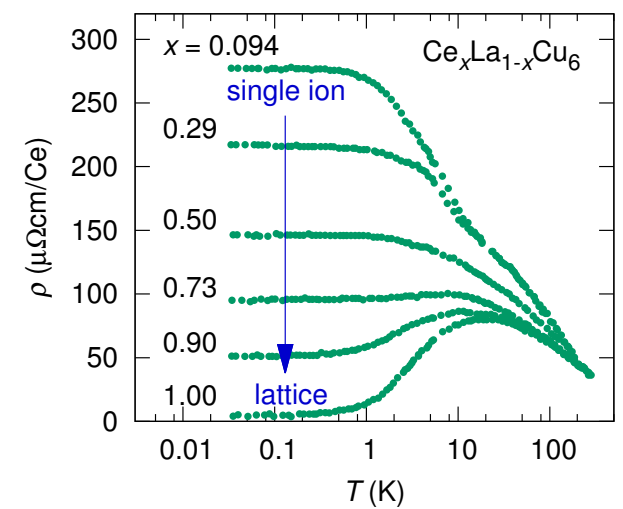

Figure 2.3: Resistivity of a Kondo system. The temperature dependence of the magnetic resistivity of $\mathrm{Ce}_{x} \mathrm{~L}_{1-x} \mathrm{Cu}_{6}$, calculated as $\rho=$ $\rho\left(\mathrm{Ce}_{x} \mathrm{La}_{1-x} \mathrm{Cu}_{6}\right)-\rho\left(\mathrm{LaCu}_{6}\right)$ per mole Cerium, is shown for different concentrations $x$. Small amounts of magnetic Ce impurities lead to a logarithmic increase towards lower $T$ until a constant value is reached below $T_{\mathrm{K}}$. This behaviour is due to the single-ion Kondo effect. As the impurity concentration is increased, a continuous development towards a coherent Kondo lattice can be observed. The resistivity exhibits a maximum before it drops towards lower $T$. Data taken from Sum86.

successfully explain the resistance minimum but becomes unphysical as $T \rightarrow 0$. The problem to resolve this divergence became known as the "Kondo problem" and renormalization group approaches [And70; Wil75] posed the first solution for it. They introduce the characteristic energy scale

$$
T_{\mathrm{K}} \propto D \mathrm{e}^{-1 / J N\left(\epsilon_{\mathrm{F}}\right)},
$$

which is called the Kondo temperature. $N\left(\epsilon_{\mathrm{F}}\right)$ is the density of states at the Fermi energy and $D$ the conduction-band width. The physics above this temperature scale in the weakcoupling regime is described by free local moments. For example, the magnetic susceptibility follows a Curie law in this temperature range. Below $T_{\mathrm{K}}$ in the strong-coupling regime, the system behaves like a non-magnetic Fermi liquid but with new composite quasiparticles. The composite quasiparticles are formed through the binding of high-energy conduction electrons to the local moment. These composite objects are then injected into the conduction band near the Fermi level, they hybridize with the conduction electrons, and form the Kondo resonance. The high effective mass $m^{*}$ of these quasiparticles leads to a strong renormalization of the Fermi-liquid properties, e.g. an enhanced specific heat and magnetic susceptibility. The resistivity does not diverge as $T \rightarrow 0$ but saturates at low temperature.

\subsubsection{Kondo Lattice}

The single-impurity Kondo model can be extended onto a regular lattice of impurities, which leads to the Kondo-lattice model. In the lattice version, the same composite fermions are formed as in the single impurity case and they also hybridize with the conduction electrons. Since there is a composite state at each site, this hybridization, however, leads now to a heavy-electron band with a large effective mass and a hybridization gap (Figure 2.2). The renormalized large Fermi surface incorporates both the conduction electrons and the degrees of freedom from the local moment. It is possible to tune systems continuously from the impurity model to the lattice model by increasing the amount of magnetic impurities, e.g. Ce in the non-magnetic host $\mathrm{LaCu}_{6}$ (Figure 2.3). While the thermodynamic properties stay essentially the same, the resistivity drops below a certain coherence temperature $T_{\text {coh }}$ when the concentration of impurities is high enough. The drop demonstrates that the scattering of the conduction electrons on the local moments becomes coherent in the Kondo lattice. 


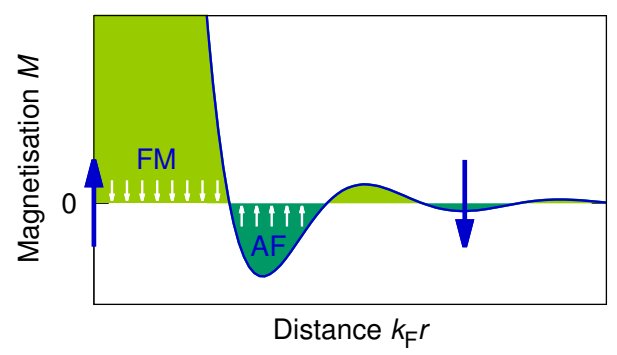

Figure 2.4: The RKKY interaction. A local moment (large arrow) induces an oscillating magnetization $M$ of the conduction electrons (small arrows) as a function of distance $r$ and Fermi wave vector $k_{\mathrm{F}}$ (cf. Equation 2.8). With the help of the polarized conduction electrons, a neighbouring local moment can couple antiferromagnetically $(\mathrm{AF})$ or ferromagnetically $(\mathrm{FM})$ to the first one.

Rare-earth electron systems are especially suited for the observation of Kondo physics for two reasons. First, the 4 -f electrons have particularly localized wave functions. Second, they show a very large spin-orbit coupling compared to the crystal electric field, which prevents a decoupling of the angular and the spin momentum $l$ and $s$. This leads to a large total angular momentum $j$ and a large degeneracy $N=2 j+1$. For example, in $\mathrm{Yb} 4 f^{13}$ systems $j=7 / 2$ and the degeneracy is $N=8$. This considerably enhances the Kondo temperature compared to the case of a simple impurity spin $S=1 / 2$. In real systems, the degenerate multiplet is split by the crystal electric field. This leads to two regimes: At higher $T$, the full multiplet takes part in the Kondo effect and gives rise to the energy scale $T_{\mathrm{K}}^{\text {high }}$, while at lower temperatures, only the ground state, typically a Kramers doublet, is involved and we find a smaller but still enhanced $T_{\mathrm{K}}^{\text {low }}$. In the following, $T_{\mathrm{K}}$ will refer to $T_{\mathrm{K}}^{\text {low }}$.

\subsubsection{RKKY Interaction and Doniach Diagram}

While the Kondo interaction favours a non-magnetic ground state, there is another phenomenon called Ruderman-Kittel-Kasuya-Yosida (RKKY) interaction, which promotes a magnetic one. This interaction must be indirect, since the wave functions of the $f$ moments are usually too localized and their overlap is too small for a direct exchange to be strong enough for any magnetic ordering. Instead, the coupling is transmitted by the conduction electrons: A magnetic moment inserted into a sea of conduction electrons induces Friedel oscillations of the spin density around its position. The magnetization $M$ of the conduction electrons oscillates with

$$
M \propto \frac{-x \cos x+\sin x}{x^{4}},
$$

where $x=2 k_{\mathrm{F}} r, k_{\mathrm{F}}$ is the Fermi wave vector, and $r$ the distance from the local moment (Figure 2.4 Blu09. A neighbouring magnetic moment can couple to this oscillating spin density, which gives rise to a long range magnetic interaction between the localized magnetic moments. The sign of the coupling depends on the distance. Its characteristic energy scale is

$$
T_{\mathrm{RKKY}} \propto J^{2} N_{\epsilon_{\mathrm{F}}},
$$

where $J$ is again the same coupling constant as the one for the Kondo effect.

Doniach pointed out that the competition between the Kondo effect and the RKKY interaction gives rise to a phase diagram as shown in Figure 2.5 Don77]. It shows the energy scales from Equation 2.7 and 2.9. For low $J N\left(\epsilon_{\mathrm{F}}\right)$, the RKKY interaction dominates, and a magnetic ground state develops, whereas the increasing Kondo energy scale leads to a 


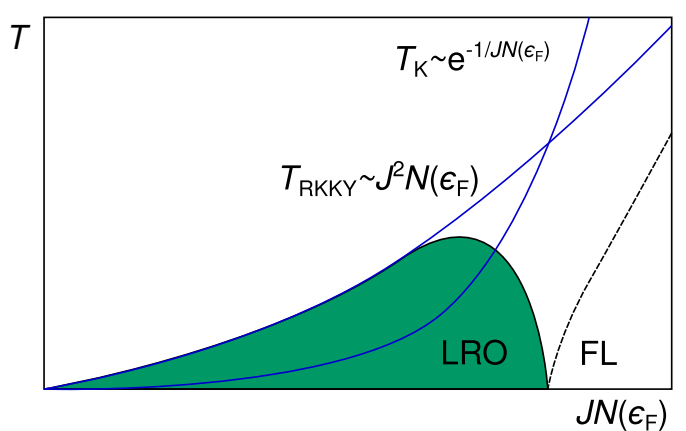

Figure 2.5: The Doniach phase diagram. The competition between the Kondo coupling $T_{\mathrm{K}}$ and the RKKY interaction $T_{\mathrm{RKKY}}$ leads to the Doniach phase diagram. At low $J N\left(\epsilon_{\mathrm{F}}\right)$, the stronger RKKY interaction induces long-range magnetic order (LRO), whereas the Kondo interaction dominates at higher values of $J N\left(\epsilon_{\mathrm{F}}\right)$ and leads to a non-magnetic Fermi-liquid (FL) ground state.

non-magnetic Fermi-liquid ground state above a critical value of $J N\left(\epsilon_{\mathrm{F}}\right)$. The transition between both ground states at $T=0$ marks a quantum critical point (QCP).

\subsection{Classical Phase Transitions}

At a classical phase transition, a system changes into an ordered state below a critical temperature $T_{\mathrm{c}}$. One usually divides phase transitions into first-order and continuous (i.e. higher-order) phase transitions. While the ordered and disordered phase coexist at $T=T_{\mathrm{c}}$ for a first-order phase transition, the order develops continuously in the latter case and is associated with an underlying broken symmetry.

The focus in this chapter is on continuous transitions. They are described by an order parameter, which is zero in the disordered phase, and finite in the ordered phase. For example, the total magnetization serves as an order parameter in a ferromagnet and it is the symmetry with respect to time reversal that is broken. However, the order parameter is fluctuating around its thermodynamic average value. Towards $T_{\mathrm{c}}$, these fluctuations grow and the correlation length $\xi$ diverges as

$$
\xi \propto|t|^{-\nu}
$$

where $\nu$ is the critical exponent of the correlation length and $t$ is a dimensionless distance from $T_{\mathrm{c}}$ (e.g. $t=\left|T-T_{\mathrm{c}}\right| / T_{\mathrm{c}}$ ). The divergence of the correlation length implies, that spacial fluctuations extend over the whole system when we approach $T_{\mathrm{c}}$. Similar fluctuations of the order parameter in the spatial dimension also exist in the time domain. Hence, we expect the correlation time $\tau_{\mathrm{c}}$ to diverge as

$$
\tau_{\mathrm{c}} \propto \xi^{z} \propto|t|^{-\nu z}
$$

where $z$ is the dynamic critical exponent. Therefore, fluctuations diverge over all length and time scales as we approach $T_{\mathrm{c}}$ - the system becomes scale invariant. Close to $T_{\mathrm{c}}, \xi$ and $\tau_{\mathrm{c}}$ are the only relevant length and time scale. As a result, the critical behaviour is described by power-law dependences of all observables on external parameters. The exponents completely characterize the system near the phase transition and they are the same for entire classes of continuous phase transitions, a phenomenon called universality. These universality classes are determined by the symmetry of the order parameter and the spatial dimensionality, but microscopic details are irrelevant. 


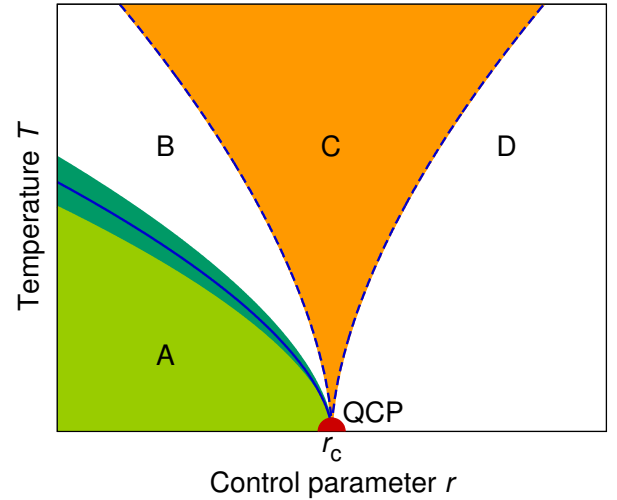

Figure 2.6: A schematic phase diagram around a QCP. The ordering temperature $T_{\mathrm{c}}$ (blue line) separates an ordered phase (A) from a disordered one. Thermal fluctuations drive the transition at finite $T$, but the regime where they dominate becomes smaller for smaller $T_{\mathrm{c}}$ (dark green). The disordered phase is separated into three regions: (B) thermally disordered, (C) quantum critical, and (D) quantum disordered, which are separated by crossovers (dashed lines).

The concept of the order parameter for classical phase transitions was first introduced by Landau. It was expanded by the leading-order term in the fluctuations of the order parameter in the Ginzburg-Landau theory. Later on, Wilson treated this concepts in a renormalization group approach, i.e., all degrees of freedom other than the order parameter fluctuations are integrated out (Landau-Ginzburg-Wilson theory).

\subsection{Quantum Phase Transitions}

Thermal fluctuations with an energy scale $k_{\mathrm{B}} T_{\mathrm{c}}$ are responsible for classical phase transitions. But in addition there are also quantum fluctuations. They exist due to Heisenberg's uncertainty principle and have the typical energy scale of $\hbar \omega_{\mathrm{c}}=\hbar / \tau_{\mathrm{c}}$. Since $\tau_{\mathrm{c}}$ diverges at a classical phase transition, this energy scale goes to zero as

$$
\hbar \omega_{\mathrm{c}} \propto|t|^{\nu z} .
$$

Thus, at any finite-temperature phase transition, quantum fluctuations will become unimportant close enough to $T_{\mathrm{c}}$ and the phase transition can be described classically.

However, one can tune a classical phase transition to $T_{\mathrm{c}}=0$ with the help of an external parameter $r, e . g$. pressure or magnetic field. In this case, the phase transition connects two ground states and its behaviour is dominated by quantum fluctuations. Such transitions are called quantum phase transition. The divergent correlation time $\tau_{\mathrm{c}}$, therefore, plays an important role in quantum phase transitions while it is less important for classical phase transitions.

These considerations lead to a phase diagram as shown in Figure 2.6. The ordered phase is separated from the disordered phase by a line of finite-temperature phase transitions tuned by the external parameter $r$. It ends at the QCP for $r=r_{\mathrm{c}}$. Classical fluctuations dominate around this line, but they extend to a narrower region as $T_{\mathrm{c}}$ becomes smaller. The disordered phase is separated into regions, which are characterized by the relevance of quantum or thermal fluctuations of the order parameter. Their boundaries are crossovers. In the thermally disordered region, long-range order is destroyed by thermal fluctuations, whereas quantum fluctuations are responsible in the quantum-disordered regime. The physics in the quantum-critical region is determined by thermal excitations of the quantum-critical 
ground state, thus it is critical in terms of $r$ but driven away from criticality in terms of $T$. The interplay of thermal and quantum fluctuations in this regime is responsible for unusual physical properties, e.g. unconventional power laws and non-Fermi-liquid behaviour.

It is possible to map a quantum phase transition onto a classical phase transition in $d_{\text {eff }}=$ $d+z$ dimensions. This allows to use the Landau-Ginzburg-Wilson formalism also for a QCP. However, there are fundamental differences between classical and quantum phase transitions especially for dynamic properties. Additional ingredients, such as coupling to fermionic modes, can also lead to qualitative differences. The latter may require the application of theories beyond Landau-Ginzburg-Wilson.

In metals, the coupling of the order-parameter fluctuations to low-energy fermionic fluctuations increases the complexity of the theoretical description. One can distinguish two cases: conventional and unconventional order parameter/quantum criticality.

In the case of conventional quantum criticality, the order-parameter fluctuations can carry a defined momentum $Q$, which couples to the fermions. For $Q=0$ (e.g. in a ferromagnet), the fluctuations can couple to fermions and create particle-hole pairs on the whole Fermi surface. The whole Fermi surface is "hot". If $Q$ is finite (e.g. in an antiferromagnet), only parts of the Fermi surface, which can be connected by $Q$, become "hot". For metals, which have a magnetically ordered phase, the case of conventional quantum criticality is often called the spin-density wave (SDW) scenario. It has theoretically been captured within an extension to the Landau-Ginzburg-Wilson formalism by Hertz Her76], Millis [Mil93, Continentino Con93, Moriya and Takimoto Mor95.

In the case of unconventional quantum criticality, the order-parameter fluctuations do not carry a specific momentum $Q$, but become critical for all momenta, hence, are local in real space. This leads to scenarios of "local criticality".

Heavy-fermion systems, which are also the focus in this work, have proven to be ideal candidates to study quantum criticality. Due to the presence of very low energy scales in these compounds, they can easily be tuned to a QCP. Examples for a SDW-type quantum critical point are $\mathrm{CeCu}_{2} \mathrm{Si}_{2}$ Sto04, Arn11; $\mathrm{Sto12b}$ and $\mathrm{CeNi}_{2} \mathrm{Ge}_{2}$ Kno88, Küc03, Kad03. Deviations from the SDW scenario and indications for local quantum criticality could be observed e.g. in $\mathrm{CeCu}_{6-x} \mathrm{Au}_{x}$ Löh07, Sto11 and $\mathrm{YbRh}_{2} \mathrm{Si}_{2}$. The criticality of the latter was studied within this work and a detailed description of previous measurement results and proposed scenarios for its magnetic-field-driven QCP are presented in Chapter 4.

\subsection{Topological Transitions}

A topology is usually classified by the number of holes. E.g. a coffee mug has the same topology as a doughnut. The relevant parameter for this classification is the Euler characteristic $\chi$. For two-dimensional polygons embedded in three dimensions with $F$ faces, $E$ edges, and $V$ vertices, this number is $\chi=F-E+V$. A cube and a pyramid, hence, have $\chi=2$. This principle can be generalized to non-polygonal surfaces.

For this purpose, we introduce the local curvature of a surface. A surface can have different curvatures depending on the direction in which it is evaluated. But at each point of the surface, there will always be a maximum curvature and a minimum curvature, which are called principal curvatures $\kappa_{\max }$ and $\kappa_{\min }$. They define how the surface looks locally: If $\kappa_{\max }$ and $\kappa_{\min }$ have the same sign, the surface looks like a dome. If one is zero, we have locally a cylinder. And if both have opposite sign, we have a saddle. Therefore we define 


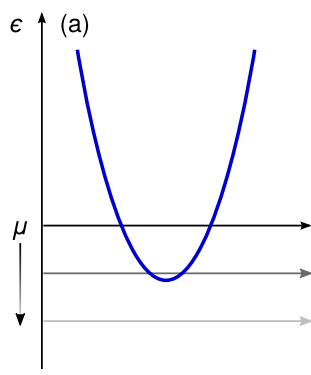

(b)

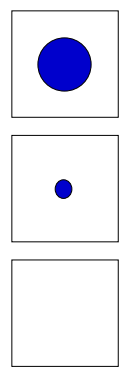

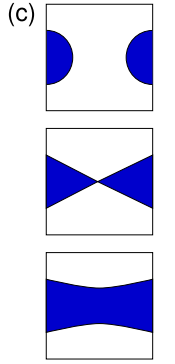

Figure 2.7: Lifshitz transition. (a) Moving the chemical potential $\mu$ through a band edge induces a Lifshitz transition of the void type. (b) In this case a Fermi-surface pocket vanishes. (c) The second most important type is a neck transition, where two Fermi surface sheets merge at the transition.

the product $K=\kappa_{\max } \kappa_{\min }$, which is the Gaussian curvature. It has been shown that

$$
\chi=\frac{1}{2 \pi} \oint_{\text {surface }} K \mathrm{~d} S
$$

which is called the Gauss-Bonnet theorem. It says that the total sum of local Gaussian curvatures at every point of the surface adds up to an invariant number, namely the Euler characteristic $\chi$, that only depends on the topology of the surface.

These general considerations, of course, also apply to Fermi surfaces in a metal. A change of the Fermi-surface topology is called Lifshitz transition. At this transition, the Euler characteristic of the Fermi surface changes discontinuously. Therefore, one can call $\chi$ the "order indicator" of a topological transition. A Lifshitz transition is a pure quantum phase transition in the sense that there is no line of finite-temperature classical critical points which terminates at the QCP. There are also no elementary excitations in terms of fluctuations of $\chi$. But there exists a non-analyticity in the ground-state energy $\Omega$ as a function of the distance to the transition $z$ Lif60]:

$$
\Omega \propto\left\{\begin{array}{ll}
0 & z<0 \\
|z|^{5 / 2} & z>0
\end{array},\right.
$$

formulated here in the case of three dimensions. In the Ehrenfest terminology, this would correspond to a phase transition of 2.5 th order.

As Figure 2.7 illustrates, a Fermi-surface change can be induced by a varying chemical potential $\mu$. The distance $z$ can thus simply be defined by $z=\mu-\epsilon_{c}$, where $\epsilon_{c}$ is the position of the transition. The two most discussed types of topological Fermi-surface changes are the void and the neck type, which are illustrated in Figure 2.7(b),(c). For the void type, a sheet of the Fermi surface vanishes (appears). In the neck type, a Fermi-surface sheet splits up into two (two sheets join).

There have been theoretical predictions how different physical quantities behave close to a Lifshitz transition and we will summarize here the results from the review by Blanter et al. Bla94 and Varlamov et al. Var89. Following those ideas, all physical quantities can be separated into a background contribution, which is a smooth function across the Lifshitz transition, and a singular part, which shows anomalies around $\epsilon_{c}$.

For the singular part of the density of states $N$, one finds in three dimensions

$$
N_{\text {singular }} \propto\left\{\begin{array}{ll}
0 & \text { Region I } \\
|z|^{1 / 2} & \text { Region II }
\end{array} .\right.
$$



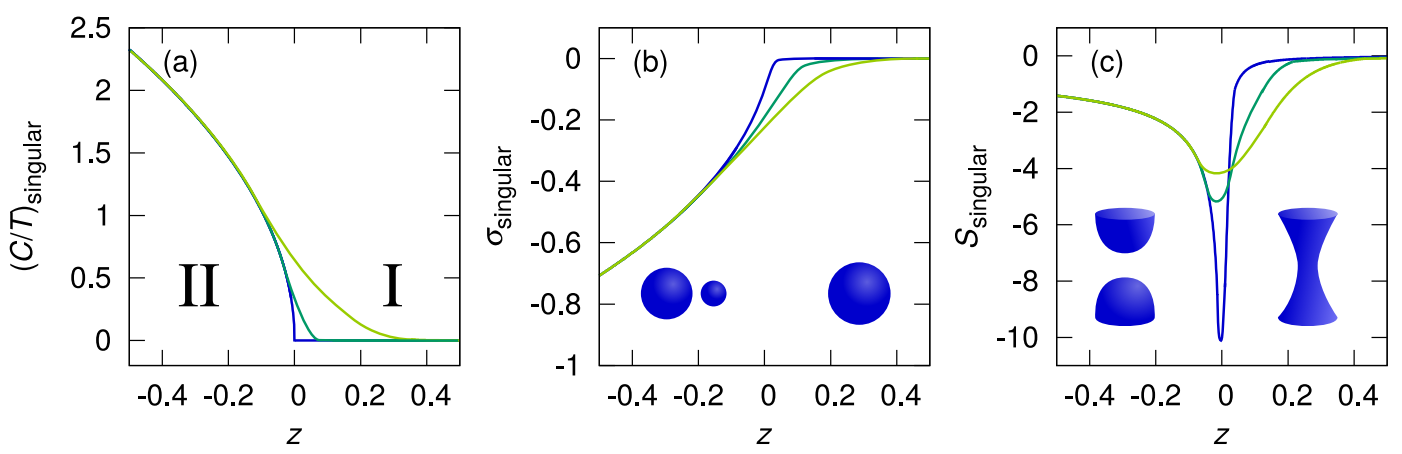

Figure 2.8: Signatures of a Lifshitz transition. The void (illustrated in (b)) and the neck transition (illustrated in (c)) show the same signatures in all quantities. At low $T$ (blue), the specific heat $C / T$ (Equation 2.16) and the conductivity $\sigma$ (Equation 2.17) show pronounced signatures in region II $(z<0)$ but are constant in region I. The thermopower $S$ (Equation 2.18), however, shows pronounced anomalies in both regions and diverges in region I. Note that the minimum in $S$ occurs right below $z=0$. All signatures are smeared for intermediate (dark green) and higher temperatures (light green). The following parameters with the same arbitrary unit are used: $\tau=10000 \hbar, \mu=1$, $T=0.01,0.04,0.07$. We considered hole carriers. The discontinuities at the limits of the different $z$ intervals in Equations 2.16 2.17 and 2.18, which are due to approximations in their derivation, are replaced by smoothly connected curves for this figure.

There are two regions left and right of the transition, which are defined by the response of the DOS: in region I, the singular part is zero in the clean limit and in region II, it is non-zero. For the void type, region I corresponds to the side of the transition where the additional Fermi surface pocket is absent, and region II to the side where it appears. For the neck type, region I corresponds to the side where the Fermi surface consists of a one-sheet hyperboloid, and regions II to the side with a two-sheet hyperboloid (illustrated in the insets of Figure 2.8.

In the free-electron picture, the singular contribution to the DOS only depends on the properties of the quasiparticles with an energy close to $\epsilon_{c}$. If we include electron-electron interactions and consider a Fermi liquid, the transition is not smeared, but now also quasiparticles away from the critical point contribute to $N_{\text {singular }}$. This changes the pre-factor of $N_{\text {singular. }}$ In contrast, elastic impurity scattering smears the transition due to a finite lifetime of the electronic states and the singular term becomes also non-zero in region I.

For the calculations of thermodynamic and transport properties, we need to distinguish four cases: $T=0$ or finite temperature; clean $(T \tau / \hbar \gg 1)$ or dirty $(T \tau / \hbar \ll 1)$ limit. $\tau$ is the scattering time. At $T=0$ and in the clean limit, one obtains for the specific heat

$$
(C / T)_{\text {singular }} \propto\left\{\begin{array}{ll}
0 & \text { Region I } \\
|z|^{1 / 2} & \text { Region II }
\end{array} .\right.
$$

For finite temperature, the singular part becomes non-zero also in region I. The behaviour for $T=0$ and the development with increasing $T$ is sketched in Figure 2.8(a). For the dirty limit, one obtains similar behaviour, however the governing energy scale is not $T$ but $\hbar \tau^{-1}$.

At finite temperature, thermal fluctuations additionally contribute to the specific heat 
and other thermodynamic quantities. They grow in the vicinity of the Lifshitz transition. However, their amplitude stays always considerably smaller than the singular parts.

Transport properties also respond to a Lifshitz transition and it is the change of the scattering time across the transition that has the largest effect on transport. For example, the most important contribution to the singular part of the conductivity in a void formation is the scattering channel between existing Fermi-surface sheets and the new void.

In contrast to thermodynamic quantities, the signatures in transport are now also smeared for $T=0$ due to impurity scattering, which needs to be taken into account. In the clean limit one finds for the singular part of the conductivity

$$
\sigma_{\text {singular }} \propto \begin{cases}(|z| / \mu)^{1 / 2} & |z| \geq T, z<0 \text { (Region II) } \\ (T / \mu)^{1 / 2} & |z| \leq T \\ (T / \mu)^{1 / 2} e^{-z / T} & T \leq z \leq T \ln (T \tau / \hbar) \text { (Region I) } \\ (\tau / \hbar)^{-1}(\mu z)^{-1 / 2} & T \ln (T \tau / \hbar) \leq z\end{cases}
$$

and of the thermopower

$$
S_{\text {singular }} \propto \begin{cases}(\mu /|z|)^{1 / 2} & |z| \geq T, z<0 \text { (Region II) } \\ (\mu / T)^{1 / 2} & |z| \leq T \\ (\mu / T)^{1 / 2}(z / T) e^{-z / T} & T \leq z \leq T \ln (T \tau / \hbar) \text { (Region I) } \\ (\mu \tau / \hbar)^{1 / 2}(z \tau)^{-3 / 2} & T \ln (T \tau / \hbar) \leq z\end{cases}
$$

The second case in each equation implies, that $S$ and $\sigma$ do not diverge at finite $T$. Again in the dirty case, the equations look similar, except that the relevant energy scale is now $\hbar \tau^{-1}$ and not $T$. The behaviour of $S_{\text {singular }}$ and $\sigma_{\text {singular }}$ as a function of $z$ and their temperature dependences are plotted in Figure 2.8(b) and (c). Especially the thermopower shows strong signatures at the transition with a rise proportional to $|z|^{-1 / 2}$ and an exponential drop afterwards. This renders the thermopower an ideal tool to detect Lifshitz transitions.

The above formulas for $\sigma_{\text {singular }}$ and $S_{\text {singular }}$ are valid for both the neck-type transition and the void type. The only difference comes in the sign of $\sigma_{\text {singular }}$, which is negative for the void type and positive for the neck type. The formulas are derived for the three-dimensional case under the assumption that the scattering angles are equally distributed. If small-angle scattering is dominant (e.g. very clean materials with electron-phonon scattering), larger differences between both types of Lifshitz transitions occur. One also expects differences between the two types in lower dimensions.

\subsection{Superconductivity}

The superconducting transition is a second-order phase transition (in zero magnetic field) and the state below the transition temperature $T_{\mathrm{c}}$ is characterized by the Meißner effect and zero electrical resistance. According to the Bardeen-Cooper-Schrieffer (BCS) theory, the electrons of a Fermi gas become unstable against an attractive potential and form Cooper pairs below $T_{\mathrm{c}}$. These pairs are bosons and condense into a state with a macroscopic wave function. This wave function is the complex order parameter of the superconducting state. The symmetry of the order parameter is intimately related to the pairing interaction. In 

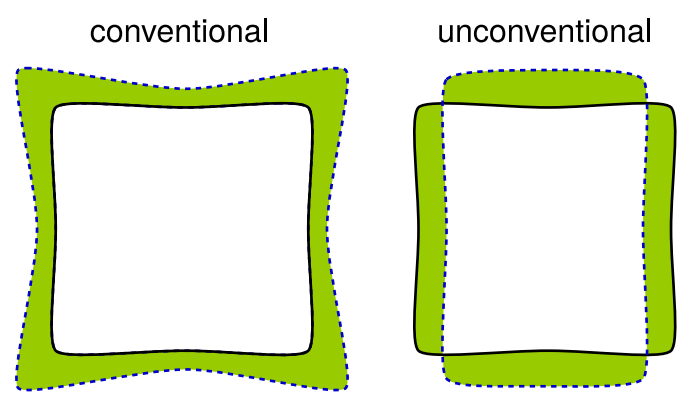

Figure 2.9: Difference between a conventional and an unconventional superconductor. The gap (dashed line) for a conventional superconductor (left) is finite everywhere around the Fermi surface (solid line), whereas it has nodes in the case of unconventional superconductors (right), which break an additional symmetry. After Min99.

order to break a Cooper pair, an energy of $2 \Delta$ is required, hence, an energy gap for a singleparticle excitation opens in the superconducting state. The gap $2 \Delta$ is proportional to the absolute value of the order parameter, and, therefore, has the same symmetry.

The wave function of a superconductor consists of a spin and an orbital part. The first determines the parity of a superconductor. It can be even $(S=0)$ or odd $(S=1)$. Additionally, superconductors are labelled $s, p, d, .$. depending on the orbital part $(l=0,1,2, \ldots)$ of the wave function. Due to the antisymmetry of the wave function with respect to particle interchange, an even (odd) parity SC can only have an $s, d, . .(p, f, .$.$) symmetry. Multicom-$ ponent order parameters are also possible and often labelled in terms like $s+d$.

Superconductors can be divided into type-I and type-II materials. In contrast to type-I superconductors, which expel a magnetic field and have only one critical field, type-II superconductors are penetrated by vortices above a lower critical field $H_{\mathrm{c} 1}$ and superconductivity breaks down only at an upper critical field $H_{\mathrm{c} 2}$. The coherence length $\xi$ and the penetration depth $\lambda$, the two parameters of the Ginzburg-Landau theory of superconductivity, are particularly useful in this context. $\xi$ determines the spatial variations of the order parameter, while $\lambda$ sets the scale to which a magnetic field penetrates the superconductor. Their ratio $\kappa_{\mathrm{GL}}$, the Ginzburg-Landau parameter, divides type-I and type-II superconductors by $\kappa_{\mathrm{GL}}<1 / \sqrt{2}$ and $\kappa_{\mathrm{GL}}>1 / \sqrt{2}$, respectively.

A second division of superconductors is by means of the symmetries which are broken below $T_{\mathrm{c}}$. In general, the symmetry group $\mathcal{H}$ of the crystal contains the gauge symmetry $U(1)$, the crystal point group $G$, the spin rotation group $S U(2)$ and the time-reversal symmetry group $\mathcal{T}$

$$
\mathcal{H}=U(1) \otimes G \otimes S U(2) \otimes \mathcal{T} .
$$

While conventional superconducting order only breaks the gauge symmetry $U(1)$, unconventional superconductors break additional symmetries besides $U(1)$. This manifests in the presence of nodes in the gap at certain wave vectors and a sign change of the order parameters at these points ( $c f$. Figure 2.9). The attractive pairing interaction in conventional superconductors is often mediated by phonons leading to a quasi-isotropic pairing potential and thus to a quasi-isotropic gap. Sometimes, "accidental nodes" are observed in conventional superconductors. As the name implies, their presence does, however, not indicate unconventional superconductivity with an additional broken symmetry besides $U(1)$. More exotic pairing mechanisms, e.g. through paramagnons, often lead to an anisotropic potential and unconventional SC. Since the gap contains information about the pairing potential, it is one of the major goals in the study of superconductors to determine its symmetry. 


\subsubsection{Properties of Conventional Superconductors}

Conventional superconductors are characterized by a finite gap in the excitation spectrum everywhere on the Fermi surface, which determines the physical properties at low temperatures. In the following, only isotropic $s$-wave gaps are considered. The absence of nodes leads to exponential behaviour in the temperature dependence of thermodynamic quantities such as the specific heat or in the electronic thermal conductivity and the penetration depth.

\section{The Gap}

The temperature dependence of the gap itself can be described by the gap equation Ann10

$$
1=\lambda_{\mathrm{ep}} \int_{0}^{\hbar \omega_{\mathrm{D}}} \mathrm{d} \epsilon \frac{1}{\sqrt{\epsilon^{2}+|\Delta|^{2}}} \tanh \left(\frac{\sqrt{\epsilon^{2}+|\Delta|^{2}}}{2 k_{\mathrm{B}} T}\right),
$$

which is the central equation of the BCS theory. $\lambda_{\mathrm{ep}}$ is the (electron-phonon) coupling parameter. The gap equation is a numerically solvable integral, whose results are tabulated e.g. in Müh59. For high $t=T / T_{c}>0.7$ one can approximate the integral by Fer64

$$
\left(\frac{\Delta(t)}{\Delta_{0}}\right)^{2}=3.016(1-t)-2.470(1-t)^{2}+0.131(1-t)^{3}+\ldots
$$

and for low $t<0.3$ by Fer64

$$
\frac{\Delta(t)}{\Delta_{0}}=1-\left[(3.562 t)^{0.5}\left(1-\frac{1.781}{8 \pi} t\right) \exp \left(-\frac{\pi}{1.781 t}\right)\right] .
$$

For an $s$-wave pairing the BCS theory predicts a gap $\Delta_{0}=\Delta(0)$ in the weak-coupling regime, which is related to $T_{\mathrm{c}}$ by

$$
\Delta_{0}=1.76 k_{\mathrm{B}} T_{\mathrm{c}} .
$$

Higher values are usually taken as a sign of strong coupling.

\section{The Specific Heat}

The specific heat $C$ can be calculated from the entropy $S$ [Tin96; Bou01],

$$
\frac{C}{\gamma_{\mathrm{n}} T_{\mathrm{c}}}=t \frac{\mathrm{d}\left(S / \gamma_{\mathrm{n}} T_{\mathrm{c}}\right)}{\mathrm{d} t}
$$

using the relation

$$
\frac{S}{\gamma_{\mathrm{n}} T_{\mathrm{c}}}=-\frac{6 \Delta_{0}}{\pi^{2} k_{\mathrm{B}} T_{\mathrm{c}}} \int_{0}^{\infty}(f \ln f+(1-f) \ln (1-f)) \mathrm{d} y,
$$

where $f=(\exp (\beta E)+1)^{-1}$ with $\beta^{-1}=k_{B} T$ determines the quasiparticle occupation number, $E=\left(\epsilon^{2}+\Delta^{2}(t)\right)^{0.5}$ are the fermionic excitation energies, $y=\epsilon / \Delta_{0}$, and $\gamma_{\mathrm{n}}$ the normal-state specific-heat coefficient. The specific heat has an exponential temperature dependence at low $T \ll \Delta_{0}$ due to the finite gap. 


\section{The Thermal Conductivity}

Bardeen, Rickayzen, and Tewordt (BRT) developed a theoretical description of the thermal conductivity for an $s$-wave superconductor within the BCS theory Bar59]. It is regarded as the standard theory for conventional superconductors.

In general, the thermal conductivity both in the normal state $\kappa^{\mathrm{n}}$ and in the superconducting state $\kappa^{\mathrm{s}}$ consists of a phonon and an electron contribution

$$
\begin{aligned}
\kappa^{\mathrm{n}} & =\kappa_{\mathrm{e}}^{\mathrm{n}}+\kappa_{\mathrm{p}}^{\mathrm{n}}, \\
\kappa^{\mathrm{s}} & =\kappa_{\mathrm{e}}^{\mathrm{s}}+\kappa_{\mathrm{p}}^{\mathrm{s}} .
\end{aligned}
$$

The electronic thermal conductivity in the superconducting state $\kappa_{\mathrm{e}}^{\mathrm{s}}$ can be calculated by

$$
\frac{\kappa_{\mathrm{e}}^{\mathrm{s}}}{\kappa_{\mathrm{e}}^{\mathrm{n}}}=\frac{f(-y)+y \ln \left(1+\mathrm{e}^{-y}\right)+y^{2} / 2\left(1+\mathrm{e}^{y}\right)}{f(0)},
$$

with

$$
y=\frac{\Delta(T)}{k_{\mathrm{B}} T}, \quad f(-y)=\int_{0}^{\infty} \frac{z \mathrm{~d} z}{1+\mathrm{e}^{z+y}},
$$

assuming dominant impurity scattering. $\Delta(T)$ is the temperature dependence of the superconducting gap as described in equation 2.21 and 2.22. This description leads to an exponential increase of $\kappa_{\mathrm{e}}^{\mathrm{s}}$ at low $T$.

Within the Debye approximation, $\kappa_{\mathrm{p}}^{\mathrm{s}}$ can be described by ( $c f$. Equation 2.47)

$$
\kappa_{\mathrm{p}}^{\mathrm{s}}=\frac{k_{\mathrm{B}}^{4} T^{3}}{2 \pi \hbar^{3} v_{\mathrm{p}}} \int_{0}^{\infty} \mathrm{d} x \frac{x^{4} \mathrm{e}^{x} \tau}{\left(\mathrm{e}^{x}-1\right)^{2}} .
$$

The scattering time $\tau$ includes a sum of different scattering mechanisms. The most common ones are

$$
\tau^{-1}=B+P x^{4} T^{4}+g(x, y) E x T,
$$

where $B, P$, and $E$ refer to scattering by crystal boundaries, point defects, and electrons. It is of course possible to include also other scattering mechanisms such as phonon-phonon scattering or scattering on other types of defects.

$g(x, y)$ is the ratio of the scattering times $\tau^{\mathrm{s}} / \tau^{\mathrm{n}}$ for electron-electron scattering in the superconducting and in the normal state. It was calculated within the BCS theory Bar59

$$
g(x, y)=\left(1-\mathrm{e}^{-x}\right) x^{-1}\left[2 J_{1}(x, y)+J_{2}(x, y)\right]
$$

with

$$
\begin{gathered}
J_{1}=\int_{\Delta(T)}^{\infty} \mathrm{d} E \frac{\epsilon^{2}+E x}{\epsilon \epsilon^{\prime}} f(E) f\left(-E^{\prime}\right), \\
J_{2}=\int_{-x+\Delta(T)}^{-\Delta(T)} \mathrm{d} E\left|\frac{E E^{\prime}}{\epsilon \epsilon^{\prime}}\right|\left(1-\frac{\Delta^{2}(T)}{E E^{\prime}}\right) f(E) f\left(-E^{\prime}\right),
\end{gathered}
$$

where $E^{2}=\epsilon^{2}+\Delta^{2}(T), E^{\prime 2}=\epsilon^{\prime 2}+\Delta^{2}(T), E^{\prime}=E+x, y=\Delta(T) / k_{B} T$, the phonon energy $x=\hbar \omega / k_{B} T$, and the Fermi function $f(E)=\left(\mathrm{e}^{E}+1\right)^{-1}$. An illustration of $g(x)$ for different $y$ is given in Tew89. The main feature is a step occurring at the phonon energy $x>2 \Delta(T)$, which is high enough to break Cooper pairs. The term $g(x, y)$ in Equation 2.31 leads to the typical increase of $\kappa_{\mathrm{p}}^{\mathrm{s}}$ below $T_{\mathrm{c}}$ due to a decrease of electronic scattering centres. 


\subsubsection{Properties of Unconventional Superconductors}

In contrast to conventional superconductors, unconventional ones may have nodes in the superconducting gap. At low $T \ll \Delta_{0}$, this leads to power-law dependences of the thermal conductivity, the penetration depth, and thermodynamic quantities like the specific heat. The power is determined by the specific gap symmetry, or more precisely by the shape of the gap around the nodes. However, it is difficult to connect an experimentally determined power with a certain gap structure, since small amounts of impurities smear the $\boldsymbol{k}$-dependence of the gap near the nodes and thus change the power in the $T$ dependence.

The specific heat, e.g., follows a $T^{2}$ behaviour for a clean $d$-wave superconductor with line nodes. These line nodes change into stripes of finite width for small amounts of impurities and the specific heat becomes linear in $T$ Min99.

\subsubsection{Multiband Superconductivity}

Many materials have a complex band structure with several bands crossing the Fermi level. Consequently, more than one band can become superconducting. This is called multiband superconductivity. The textbook example is $\mathrm{MgB}_{2}$. Simple theoretical descriptions to model experimental data are only available for the case of isotropic $s$-wave gaps with a BCS-like temperature dependence. In the following, we will discuss the $\alpha$ model for the case of two bands Bou01.

The phenomenological $\alpha$ model assumes two isotropic $s$-wave gaps $\Delta_{1}$ and $\Delta_{2}$ both closing at $T_{c}$. The contribution of each single gap to the specific heat is calculated within the BCS theory assuming $\Delta_{0}$ in Equation 2.25 not to be fixed to the BCS value of 1.76. In practice, $\Delta_{1}$ and $\Delta_{2}$ instead serve as fitting parameters along with a weighting factor $x_{1}$, which determines the total specific heat as

$$
\frac{C_{\mathrm{s}}}{\gamma_{\mathrm{n}} T_{c}}=x_{1} \frac{C_{1}}{\gamma_{\mathrm{n}} T_{c}}+\left(1-x_{1}\right) \frac{C_{2}}{\gamma_{\mathrm{n}} T_{c}} .
$$

$\gamma_{\mathrm{n}}$ refers to the normal-state specific-heat coefficient, $C_{\mathrm{s}}$ to the total specific heat in the superconducting state.

Due to its simplicity, the $\alpha$ model is the most widely used model to describe experimental data on multiband superconductors.

\subsection{Transport in Solids}

In a semi-classical picture, transport in a solid is described by the motion of quasiparticles, e.g. electrons, phonons or magnons, which is interrupted by scattering off defects or off each other. The generalized transport equations

$$
\begin{aligned}
& \boldsymbol{j}_{\boldsymbol{e}}=\hat{L}_{11} \boldsymbol{E}+\hat{L}_{12} \nabla T, \\
& \boldsymbol{j}_{\boldsymbol{q}}=\hat{L}_{21} \boldsymbol{E}+\hat{L}_{22} \nabla T,
\end{aligned}
$$

describe transport as the linear response of the thermal and the electrical current $\boldsymbol{j}_{\boldsymbol{q}}$ and $\boldsymbol{j}_{\boldsymbol{e}}$ to an electrical and thermal gradient $\boldsymbol{E}$ and $\nabla T$. The tensors $\hat{L}_{i j}$ transform into the known 
transport tensors

$$
\hat{\sigma}=\hat{L}_{11}, \quad \hat{\alpha}=-\hat{L}_{12}, \quad \hat{\kappa}=-\hat{L}_{22}+\frac{\hat{L}_{21} \hat{L}_{12}}{\hat{L}_{11}},
$$

where $\hat{\sigma}$ is the electrical-conductivity tensor and $\hat{\kappa}$ the thermal-conductivity tensor. From $\hat{\alpha}$ and $\hat{\sigma}$, we derive the thermoelectric tensor

$$
\hat{S}=-\hat{\sigma}^{-1} \hat{\alpha}
$$

Although all these are $3 \times 3$ tensors, the number of their elements reduces considerably for certain symmetries. In the case of a magnetic field $\boldsymbol{H}=\left(0,0, H_{z}\right)$ applied perpendicular to the currents and with the additional help of Onsagers relations we arrive at

$$
\hat{\sigma}=\left(\begin{array}{cc}
\sigma_{x x} & \sigma_{x y} \\
-\sigma_{x y} & \sigma_{y y}
\end{array}\right), \quad \hat{\kappa}=\left(\begin{array}{cc}
\kappa_{x x} & \kappa_{x y} \\
-\kappa_{x y} & \kappa_{y y}
\end{array}\right), \quad \hat{\alpha}=\left(\begin{array}{cc}
\alpha_{x x} & \alpha_{x y} \\
-\alpha_{x y} & \alpha_{y y}
\end{array}\right)
$$

The single elements correspond to the usually measured physical properties: $\sigma_{i i}$ is the electrical conductivity, $\sigma_{i j}$ is the Hall conductivity, $\kappa_{i i}$ the thermal conductivity and $\kappa_{i j}$ the thermal Hall conductivity. Care has to be taken to correctly calculate the resistivities from the above matrices. The thermopower becomes

$$
S_{x x}=\frac{\sigma_{y y} \alpha_{x x}-\sigma_{x y} \alpha_{x y}}{\sigma_{x x} \sigma_{y y}+\sigma_{x y}^{2}}
$$

for the $x$ direction. The off-diagonal elements of $\hat{S}$ refer to the Nernst coefficient. For a field parallel to the currents, all off-diagonal elements in Equation 2.39 become zero, i.e., we do not observe e.g. a Hall effect.

The currents in the transport equations 2.36 can be expressed as an integral of the velocity $\boldsymbol{v}_{\boldsymbol{k}}$ and the distribution function $f_{\boldsymbol{k}}$ paired with the charge $e$ or the energy $\epsilon_{\boldsymbol{k}}$

$$
\begin{aligned}
& \boldsymbol{j}_{\boldsymbol{e}}=\frac{2}{(2 \pi)^{3}} \int e \boldsymbol{v}_{\boldsymbol{k}} f_{\boldsymbol{k}} \mathrm{d}^{3} \boldsymbol{k}, \\
& \boldsymbol{j}_{\boldsymbol{q}}=\frac{2}{(2 \pi)^{3}} \int \epsilon_{\boldsymbol{k}} \boldsymbol{v}_{\boldsymbol{k}} f_{\boldsymbol{k}} \mathrm{d}^{3} \boldsymbol{k} .
\end{aligned}
$$

To determine the transport coefficients, the distribution function $f_{\boldsymbol{k}}$ remains to be calculated, which is often done within the Boltzmann formalism.

\subsubsection{Boltzmann Equation}

The simplest approach to transport phenomena is the semi-classical Boltzmann theory. It can be used to describe transport in solids due to particles with well-defined position and momentum. The Boltzmann equation

$$
\left(\frac{\partial}{\partial t}+\frac{\boldsymbol{p}}{m} \frac{\partial}{\partial \boldsymbol{r}}+\boldsymbol{F} \frac{\partial}{\partial \boldsymbol{p}}\right) f(\boldsymbol{r}, \boldsymbol{p}, t)=S[f]
$$

consists of a part representing a continuity equation for the phase-space distribution $f$ on the left and the scattering functional $S[f]$ on the right. In the stationary case the first term 
$\partial f / \partial t$ vanishes. The second term is called the drift term and describes changes of $f$ due to gradients, e.g. for a thermal gradient

$$
\frac{\boldsymbol{p}}{m} \frac{\partial f}{\partial \boldsymbol{r}}=\frac{\boldsymbol{p}}{m} \frac{\partial}{\partial \boldsymbol{r}} T \frac{\partial f}{\partial T}
$$

The third term describes external forces, e.g. electromagnetic fields. The most complicated term is the scattering functional $S[f]$, which is mostly treated in the relaxation-time approximation

$$
S[f]=\frac{f(\boldsymbol{r}, \boldsymbol{p}, t)-f^{0}(\boldsymbol{r}, \boldsymbol{p}, t)}{\tau} .
$$

This approximation forces $f$ to converge towards an equilibrium distribution $f_{0}$ within a time scale $\tau$. $f_{0}$ is the usual Fermi or Dirac distribution, $\tau$ the scattering time. For $N$ different scattering mechanisms, the single $\tau_{i}$ may be summed by Matthiessens rule

$$
\tau^{-1}=\sum_{i=1}^{N} \tau_{i}^{-1} .
$$

With the relaxation-time approximation, the Boltzmann equation turns into a linear partial differential equation. To calculate transport in solids, the phase-space distribution $f(\boldsymbol{r}, \boldsymbol{p}, t)$, which describes classical particles, can be replaced by the quantum-mechanical distribution function $f_{\boldsymbol{k}}$ for quantum-mechanical particles. This procedure is justified by the WignerWeyl transformation.

\subsubsection{Thermal Conductivity}

Within the Boltzmann formalism one can derive the following formula for the thermal conductivity

$$
\kappa=\frac{1}{d} \frac{1}{(2 \pi)^{d}} \int c_{\boldsymbol{k}} v_{\boldsymbol{k}} l_{\boldsymbol{k}} \mathrm{d} \boldsymbol{k},
$$

where $d$ is the dimension of the corresponding quasiparticle system. The related simple formula $\kappa=C v l / 3$ is often used to roughly estimate the thermal conductivity. $C$, $v$, and $l$ are the specific heat, the velocity and the mean free path of the corresponding quasiparticle, which transports the energy current.

Different quasiparticles can contribute to the thermal transport. Along with defects, all of them also serve as scattering centres. Therefore, the separation of the single contributions to $\kappa$ turns out to be difficult in practice. Additionally, the theoretical description of thermal transport is generally more complex than for electrical transport with only one contribution from electrons. Simple temperature dependences can only be derived under certain assumptions. More complex scattering mechanisms or excitation spectra considerably alter these dependences or even render a theoretical description impossible.

\section{Phonon contribution}

In a simple approximation, lattice vibrations are described with a harmonic potential. Together with the Debye model and the relaxation-time approximation, one can derive the 

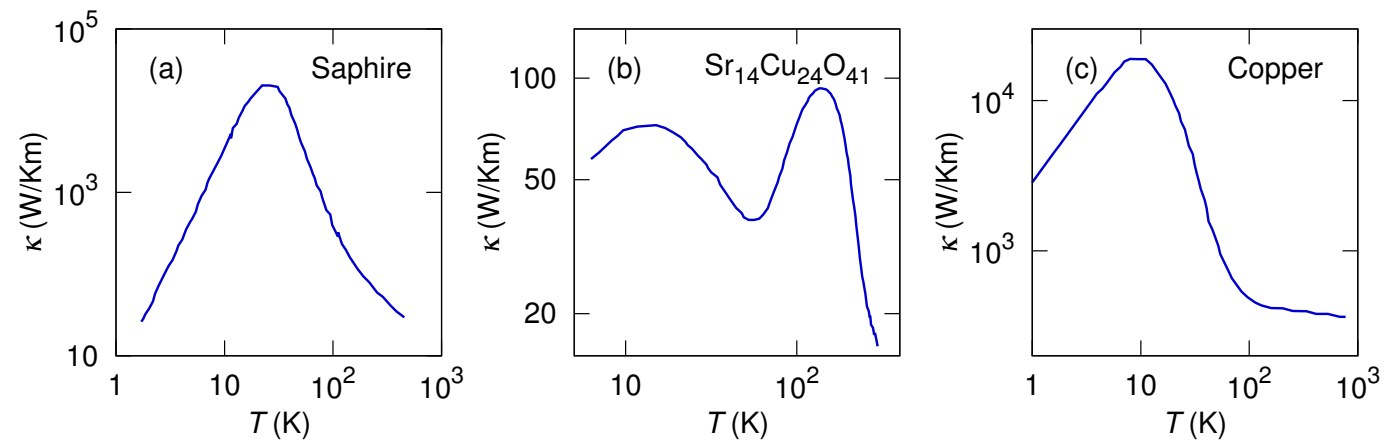

Figure 2.10: Textbook examples for the thermal conductivity by phonons, magnons, and electrons. The thermal conductivity $\kappa$ as a function of temperature $T$ is plotted in a double-logarithmic way for (a) sapphire after Tou70, (b) $\mathrm{Sr}_{14} \mathrm{Cu}_{24} \mathrm{O}_{41}$ after Hes01, and (c) copper after Tou70. Sapphire is a typical phonon conductor, while $\mathrm{Sr}_{14} \mathrm{Cu}_{24} \mathrm{O}_{41}$ shows a phonon and a magnon contribution as maxima at lower and higher $T$, respectively. The thermal conductivity in copper is dominated by electronic transport.

following formula for the phonon thermal conductivity:

$$
\kappa_{\mathrm{ph}}=\frac{k_{\mathrm{B}}}{2 \pi^{2} v}\left(\frac{k_{\mathrm{B}}}{\hbar}\right)^{3} T^{3} \int_{0}^{\Theta / T} \tau(x) \frac{x^{4} \mathrm{e}^{x}}{\left(\mathrm{e}^{x}-1\right)^{2}} \mathrm{~d} x .
$$

To sketch the temperature dependence of the phonon thermal conductivity, we will use the more simple relation $\kappa_{\mathrm{ph}}=\mathrm{Cvl} / 3$, derived from Equation 2.46 assuming a $k$-independent mean free path and velocity. Following this equation, we can distinguish two regimes: At low temperatures compared to the Debye temperature $\Theta$, umklapp scattering is frozen out and the mean free path becomes constant, limited only by scattering on crystallite and sample boundaries. $\kappa_{\mathrm{ph}}$ thus follows the specific heat with a $T^{3}$ dependence. At high temperatures, umklapp scattering leads to a mean free path $l \propto T^{-1}$. The specific heat becomes constant and $\kappa_{\mathrm{ph}}$ follows $T^{-1}$. This behaviour can be observed e.g. in sapphire, which is sketched in Figure 2.10 (a). Scattering due to electrons or other quasiparticles is not included here.

\section{Contribution due to magnetic excitations}

Magnetic excitations, like phonons or electrons, carry entropy and thus can transport heat. This was theoretically predicted quite early [Frö36] and observed experimentally on YttriumIron-Garnet in the nineteen sixties Dou63. The observation of large magnetic contributions to the heat conduction in the low-dimensional spin systems of the cuprates Sol00: Hes01 and the prediction of ballistic transport in integrable one-dimensional models Zot97: Zot99 inspired the research on magnetic heat transport.

Due to the large number of magnetic structures, a comparable large number of models exists to describe their ground states and excitations. The main differences lie between fermionic spin excitations called spinons and bosonic excitations called magnons. In a simple approximation, magnons without a gap in their excitation spectrum can be treated in 
an adapted Debye model leading to the same temperature dependences as for phonons at low temperatures. But in contrast to phonons, magnons usually show a magnetic-field dependence. Spinons, since they are fermions, do not freeze out at zero temperature unlike magnons. Therefore, they can contribute to the thermal transport $\kappa / T$ at $T=0$ similarly to electrons.

An example for magnon transport, where its contribution can be nicely separated from the phonon contribution, is found in the spin-ladder compound $\mathrm{Sr}_{14} \mathrm{Cu}_{24} \mathrm{O}_{41}$, which is sketched in Figure 2.10(b).

\section{Electron contribution}

The thermal conductivity in metals is dominated by the contribution of electrons. In the free-electron picture the thermal conductivity again becomes $\kappa_{\mathrm{el}}=C v_{\mathrm{F}} l / 3$, with the Fermi velocity $v_{\mathrm{F}}$. As seen in Equation 2.3 the specific heat is proportional to $T$. Considering electron-phonon scattering, the scattering rate $\tau^{-1}=v_{\mathrm{F}} / l$ and the thermal conductivity shows the following dependence

$$
\tau^{-1} \sim\left\{\begin{array}{ll}
\text { const. } & T \ll \Theta \\
T^{3}, & T<\Theta \\
T, & T>\Theta
\end{array}, \quad \kappa_{\mathrm{el}} \sim\left\{\begin{array}{ll}
T, & T \ll \Theta \\
T^{-2}, & T<\Theta . \\
\text { const. } & T>\Theta
\end{array} .\right.\right.
$$

This can be observed, e.g., in copper as shown in Figure 2.10(c).

\subsubsection{Wiedemann-Franz Law}

Let us consider transport due to electrons and compare the ratio of the thermal and electrical conductivity

$$
L=\frac{\kappa}{T \sigma} .
$$

$L$ is called the Lorenz number. An important prediction of the Fermi-liquid theory is that $L=L_{0}$ at $T=0$, where $L_{0}=2.44 \cdot 10^{-8} \mathrm{~W} \Omega \mathrm{K}^{-2}$ is Sommerfeld's constant. This is called the Wiedemann-Franz (WF) law. This fundamental physical law is, however, not only valid in a Fermi liquid, but generally in systems, which can be described in the framework of quasiparticles. Some non-Fermi liquids also belong to this class such as systems with a conventional quantum critical point.

The following considerations together with Figure 2.11 offer an instructive access to the WF law: Applying a gradient, the distribution of the electrons is changed in a different way for a thermal and an electrical gradient as illustrated in Figure 2.11. Scattering processes tend to relax the distribution back to equilibrium. One distinguishes different types of scattering processes: elastic and inelastic as well as horizontal (large-angle) and vertical (small-angle) processes.

An elastic process scatters an electron to another position on the same constant-energy surface. In the framework of the linearised Boltzmann equation, the scattering times for the electrical and the thermal conductivity are the same in this case. Hence, $L=L_{0}$ for elastic scattering. From Figure 2.11 it becomes clear that large-angle elastic scattering has the same effect on both, the thermal and the electrical current. Small-angle elastic scattering has only little impact on the distributions. An example of elastic processes is scattering 


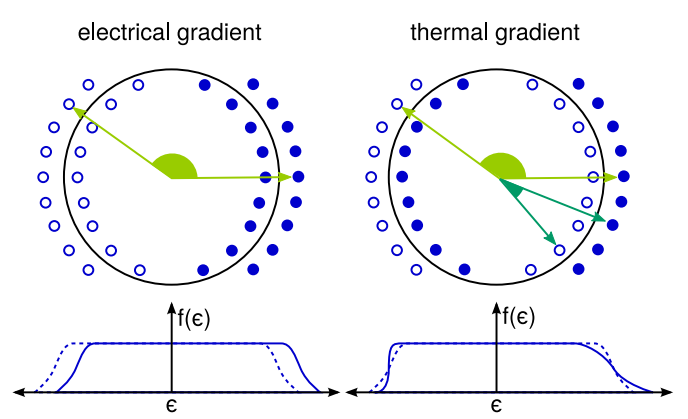

Figure 2.11: Effects of a thermal (right) and an electrical (left) gradient on the excitations around the Fermi surface (top) and the distribution function $f(\epsilon)$ (bottom). Closed (open) dots represent occupied (unoccupied) states when a gradient is applied. Large-angle scattering (bright green) is able to relax excitations in both cases, whereas small-angle scattering (dark green) is only efficient for thermal gradients. $f(\epsilon)$ with (without) an applied gradient is represented by a solid (dotted) line. After Zim60].

off defects, which is the only mechanism acting at very low temperatures $T \ll \Theta$. "Quasielastic" large-angle processes occur at large temperatures $T>\Theta$ due to phonons, which have a comparably small energy but large wave vectors in this regime.

In contrast, small-angle inelastic scattering is very efficient to reduce the thermal but not the electrical current. In this case, the WF law no longer holds. An example is electronphonon scattering at low temperatures $T<\Theta$.

\subsubsection{Thermopower}

A very sensitive transport property in a metal is its thermopower $S$. From the Boltzmann equation it can be expressed by the energy derivative of the conductivity at the Fermi energy

$$
S=\frac{\pi^{2} k_{\mathrm{B}}^{2} T}{3 e}\left(\frac{\partial \ln \sigma(\epsilon)}{\partial \epsilon}\right)_{\epsilon_{\mathrm{F}}},
$$

which is called Mott formula. $e$ denotes the elementary charge and $\epsilon$ the energy. In a Fermi liquid, the thermopower usually becomes linear in $T$ at low temperatures. The derivative in the above equation can be translated using $\sigma=e^{2} l \mathcal{A} / 12 \pi^{3} \hbar$, where $\mathcal{A}$ is the Fermi-surface area, into Zim60

$$
\frac{\partial \ln \sigma(\epsilon)}{\partial \epsilon}=\frac{\partial \ln l(\epsilon)}{\partial \epsilon}+\frac{\partial \ln \mathcal{A}(\epsilon)}{\partial \epsilon},
$$

which demonstrates the sensitivity of the thermopower to Fermi-surface properties.

The Mott formula 2.50 can also be expanded in a different way using the simple freeelectron picture $\sigma=n e^{2} \tau / m^{*}$, together with $m^{*} \propto N^{2 / 3}$,

$$
\frac{\partial \ln \sigma(\epsilon)}{\partial \epsilon}=\frac{\partial \ln \tau}{\partial \epsilon}-\frac{2}{3} \frac{\partial \ln N}{\partial \epsilon},
$$

where $n$ is the total electron concentration, $N$ the density of states and $\tau$ the scattering time. This version demonstrates the large sensitivity of the thermopower to the energy dependence of the DOS. It also provides a simple picture to understand the large values of the thermopower in heavy-fermion compounds. They usually have a Kondo resonance in the DOS slightly below (for holes) or above (for electrons) the Fermi energy. At low enough temperatures, the steep slope of $N$ at $\epsilon_{\mathrm{F}}$ leads to large values of $S$ with the opposite sign as expected from the charge of the carriers. 
In the case of a multi-band system, the contributions to the thermopower from the single bands $S_{i}$ sum up as

$$
\sigma S=\sum_{i} S_{i} \sigma_{i}
$$

Different scattering contributions are accounted for by the Gorter-Nordheim rule

$$
\rho S=\sum_{i} S_{i} \rho_{i}
$$

which is derived under the assumption, that the Wiedemann-Franz law holds.

The thermopower in form of the Mott formula describes only one contribution to $S$, namely the diffusion term. It was derived under the assumption that the phonon system stays in equilibrium. However a thermal gradient always gives rise to heat transport by phonons and an electric current transfers momentum to the phonon system. This leads to the so-called phonon drag effect and an additional contribution to the thermopower. In the compounds and temperature ranges studied throughout this thesis, this effect and also similar effects due to magnons are insignificant. 



\section{Experimental Techniques for
Transport Measurements}

The focus of this thesis in terms of experimental techniques lies on thermopower and thermal-conductivity measurements in a dilution refrigerator at temperatures below $1 \mathrm{~K}$. This chapter provides a general description of this method as well as the specifics of the three different set-ups in which the experiments where performed. In this context, I will also refer to the possibility to measure in-situ the electrical resistivity in two of these set-ups. Additional measurements of thermal transport at higher temperatures and of other quantities were performed with a commercially available Physical Property Measurement System (PPMS). A detailed description of its capabilities and the methods can be found at Qua99a Qua99b Qua99c.

\subsection{General Principle of Thermal Transport in a Dilution Refrigerator}

The standard two-thermometers-one-heater set-up to measure thermal transport is illustrated in Figure 3.1. To determine the thermal conductivity, a heater with the power $P_{\mathrm{H}}$ generates a thermal gradient $\Delta T=T_{2}-T_{1}$, which is measured by two thermometers $(1,2)$. The thermal conductivity $\kappa$ is then

$$
\kappa=\frac{l_{\mathrm{S}}}{A_{\mathrm{S}}} \frac{P_{\mathrm{H}}}{\Delta T},
$$

where $l_{\mathrm{S}}$ is the distance between which $\Delta T$ is measured and $A_{\mathrm{S}}$ the cross section of the sample. To determine the thermopower

$$
S=\frac{\Delta U_{\mathrm{T}}}{\Delta T}
$$

two additional thermovoltage leads are attached to the same contacts as the thermometers. They measure the thermovoltage $\Delta U_{\mathrm{T}}$ across the sample. The thermopower $S$ and the thermal conductivity $\kappa$ can be measured simultaneously. The configuration in Figure 3.1 offers the opportunity to measure the electrical resistivity $\rho$ as well. While it has to be determined in a separate run, advantageously it is not necessary to warm up the whole cryostat. For the electrical resistivity

$$
\rho=\frac{A_{\mathrm{S}}}{l_{\mathrm{S}}} \frac{\Delta U_{\mathrm{res}}}{I_{\mathrm{res}}},
$$


the contacts to the heater and to the sample holder are used to apply an electrical current $I_{\text {res }}$, the voltage drop $\Delta U_{\text {res }}$ is measured in the same way as for the thermopower. This configuration ensures the same geometry factor $l_{\mathrm{S}} / A_{\mathrm{S}}$ for $\kappa$ and $\rho$, which is important for an accurate determination of the Lorenz ratio for the Wiedemann-Franz law.

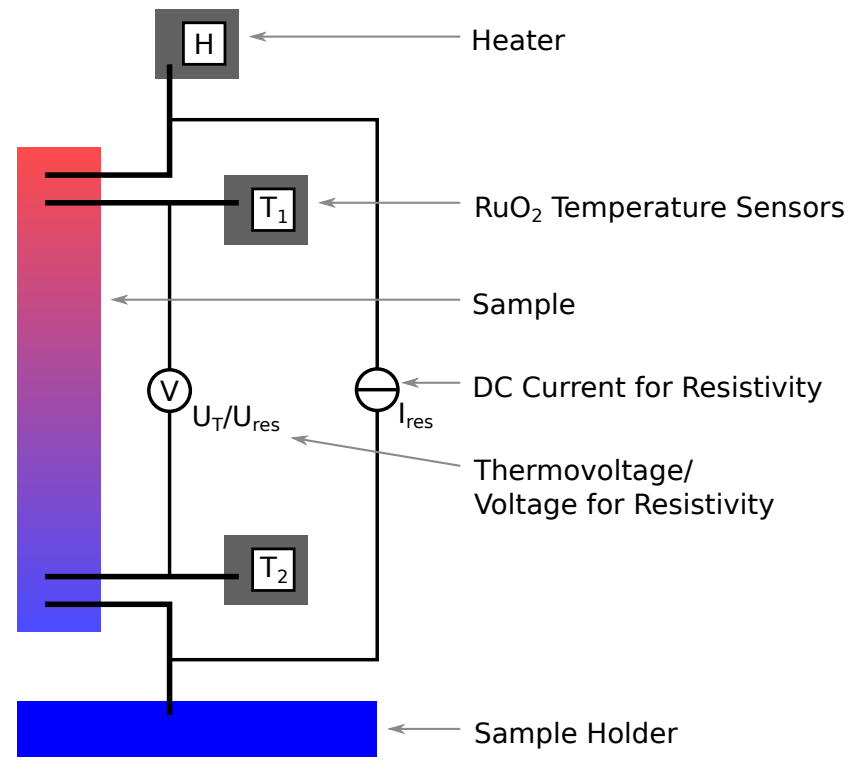

Figure 3.1: Scheme of the two-thermometers-one-heater measurement set-up.

\subsection{The Three Set-ups Used}

The general principle explained above applies for all set-ups, however, the specific layout and the materials differ a little. Three different set-ups were used on three different refrigerators, which we will call "Franz" (F), "Rotator" (R), and "SHE" (S). Table 3.1 summarizes the important specifications of the three set-ups. "Franz" is used for measurements where it is important to reach temperatures as low as possible. The "SHE" is used for the highest fields. The "Rotator" offers two magnets - a horizontal and a vertical one. Since the cryostat including the magnet coils can rotate around the fixed insert of the dilution refrigerator, this set-up is used for measurements with variable field directions.

The Figure 3.2 shows photographs of the sample holders for the "SHE" and the "Franz" set-up. The one for the "Rotator" looks similar to the latter. A very detailed description of the sample holders for the "Rotator" and for "Franz" can be found in Vie11 and Har10a, respectively. At the "Rotator", only thermal conductivity can be measured. At "Franz", a measurement of the thermal conductivity, the thermopower and the resistivity is possible. The "SHE" additionally provides the possibility to measure Nernst and thermal Hall effect.

The devices used for the measurement are almost identical for all three set-ups and are summarized in Table 3.2 . 


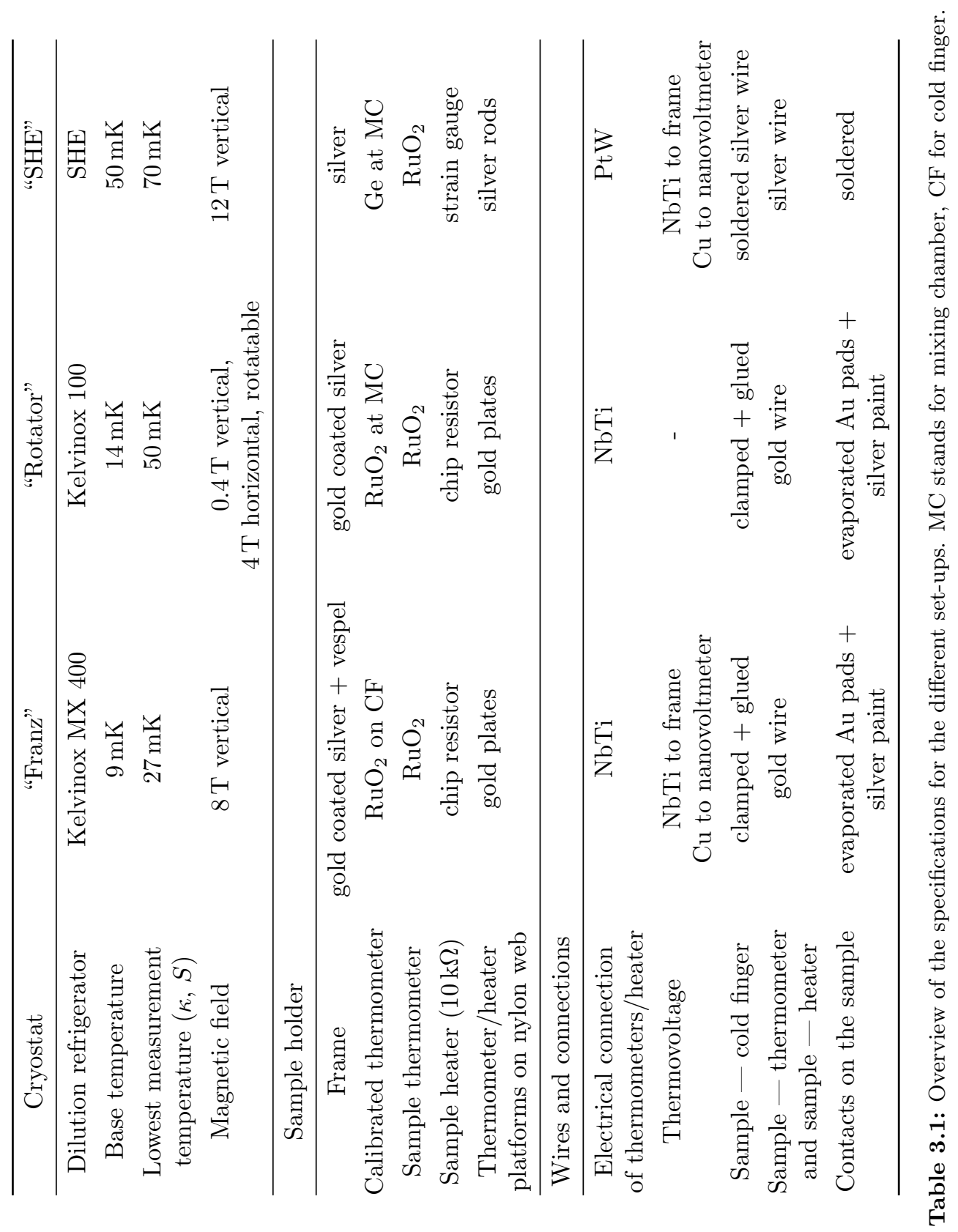



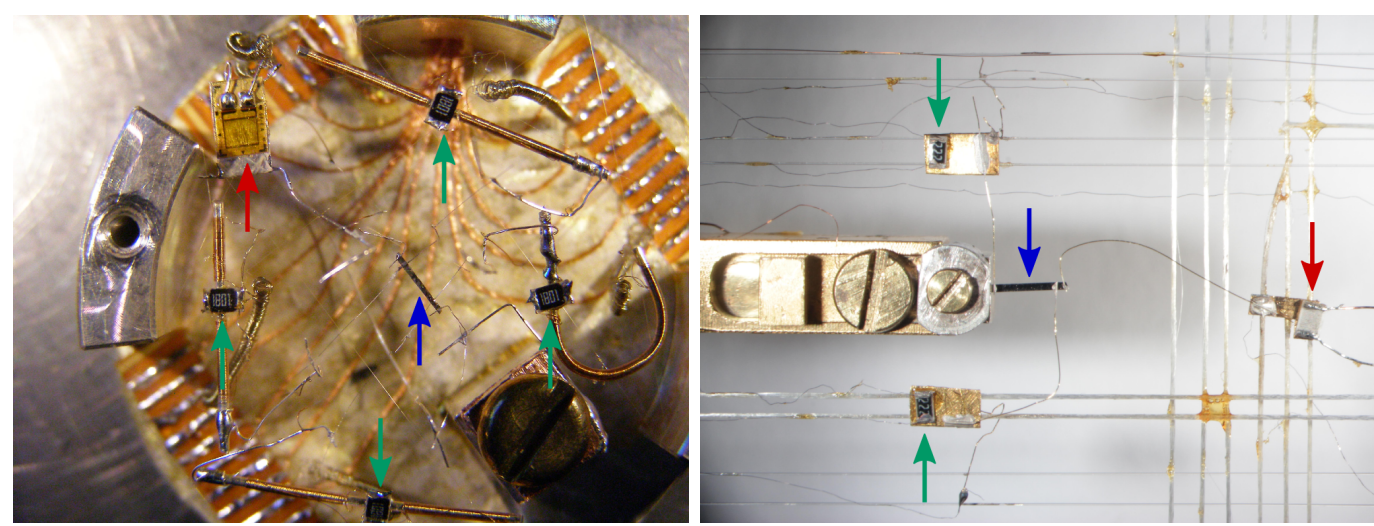

Figure 3.2: Sample holders of "SHE" (left) and "Franz" (right). The sample holder of the "Rotator" set-up looks similar to the "Franz", however it does not contain thermovoltage leads. The arrows mark the sample (blue), the sample thermometers (green) and the sample heater (red).

\begin{tabular}{cc}
\hline Quantity & Device \\
\hline Thermometer resistances & LakeShore Resistance Bridge LS370 \\
and temperature control & + LakeShore Scanner Model 3716 \\
Sample heater current & Keithley $6220(\mathrm{~S})$, Keithley 224 (R), Keithley 263 (F) \\
Sample heater voltage & Nanovoltmeter Keithley 2182 \\
Thermovoltage & EM D.C. Nanovolt Amplifier Model A14 \\
& + Keithley Multimeter 2001 (S), \\
& EM D.C. Picovoltmeter Model P13 \\
& + Keithley Nanovoltmeter 2182 (F) \\
\hline
\end{tabular}

Table 3.2: Overview of the devices used at the different set-ups. F, S, and R refer to "Franz", "SHE", and "Rotator".

\subsection{Measurement Procedure-Steady State}

For the standard steady-state method illustrated in Figure 3.3, the temperature of the sample holder $T_{\mathrm{MC}}$ is stabilized using the calibrated thermometer which sits either on the cold finger or the mixing chamber. The values of the sample thermometer resistances $R_{1,2}$ and the voltage between the thermovoltage leads $U_{\mathrm{T}}$ are then detected in both states, when the sample heater is switched off and on. The first values of the sample-thermometer resistances $R_{1,2}\left(P_{\mathrm{H}}=0\right)$ serve as calibration points for the thermometers, the latter are used to determine the thermal gradient $\Delta T$. The thermovoltage $\Delta U_{\mathrm{T}}$ is the difference between the background measurement, i.e., in the heater off state, and the points when the heater is switched on $\Delta U_{\mathrm{T}}=U_{\mathrm{T}}\left(P_{\mathrm{H}} \neq 0\right)-U_{\mathrm{T}}\left(P_{\mathrm{H}}=0\right)$. This procedure is normally repeated first with several different values of the sample heater power $P_{\mathrm{H}}=U_{\mathrm{H}} I_{\mathrm{H}}$ to check for linear response. Afterwards, the above procedure is repeated with different $T_{\mathrm{MC}}$. The mean value of the temperatures $T_{1}$ and $T_{2}$ during the heater on stage is taken as the average sample 


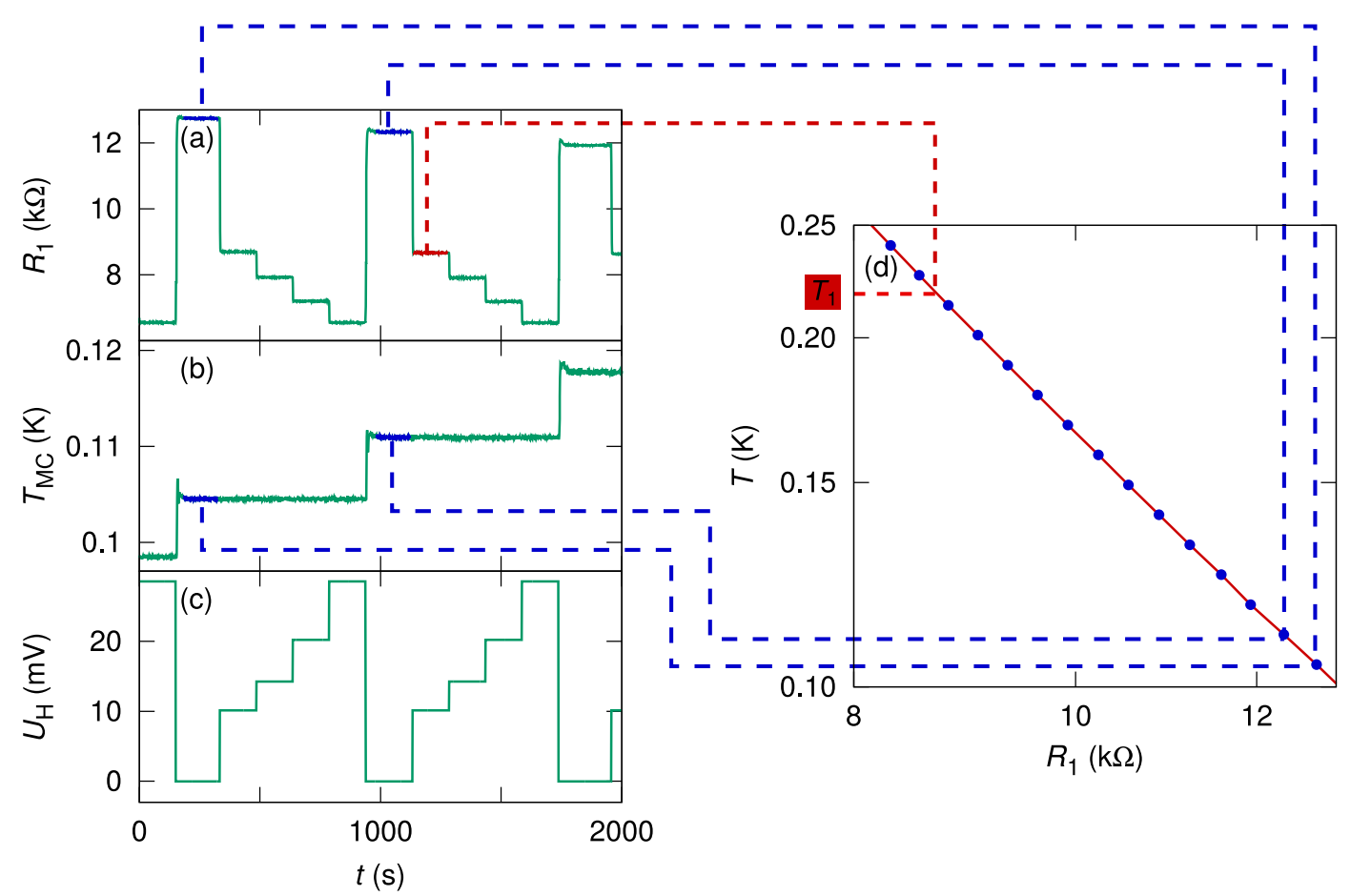

Figure 3.3: Measurement procedure for the steady-state method. When the heater is off $\left(U_{H}=0\right.$ in panel (c)), the points from the sample thermometer resistance $R_{1}$ (a) and the mixing chamber/cold finger temperature $T_{\mathrm{MC}}$ (b) are used to create the calibration curve $T\left(R_{1}\right)$ of the sample thermometer 1 shown as blue dots in panel $(\mathrm{d})$. The calibration function is a linear interpolation between neighbouring points (red line in (d)). For the heater-on states, the sample temperature $T_{1}$ can be calculated from $R_{1}$ using this calibration function. The same procedure applies for $T_{2}$.

temperature $T$ and is used for all plots shown throughout this thesis. It is useful to apply several different gradients for one $T_{\mathrm{MC}}$, because it is often faster to change the sample temperature by a different applied gradient than by a different $T_{\mathrm{MC}}$. Therefore, it reduces the time per measured $\kappa(T)$ value.

The in-situ calibration of the sample thermometers is necessary because their characteristics change in magnetic field and due to mechanical stress e.g. after changing the sample.

The same principle is used for the steady-state resistivity measurements. The thermal current produced by the sample heater is replaced by an electrical current from a current source.

\subsection{Measurement Procedure-Pseudo AC}

The pseudo AC method, illustrated in Figure 3.4, is used for isothermal measurements, which depend on an external parameter $x$, e.g. magnetic field or magnetic-field angle. For the simplest measurement of this type, a fixed sample holder temperature $T_{\mathrm{MC}}$ is stabilized 


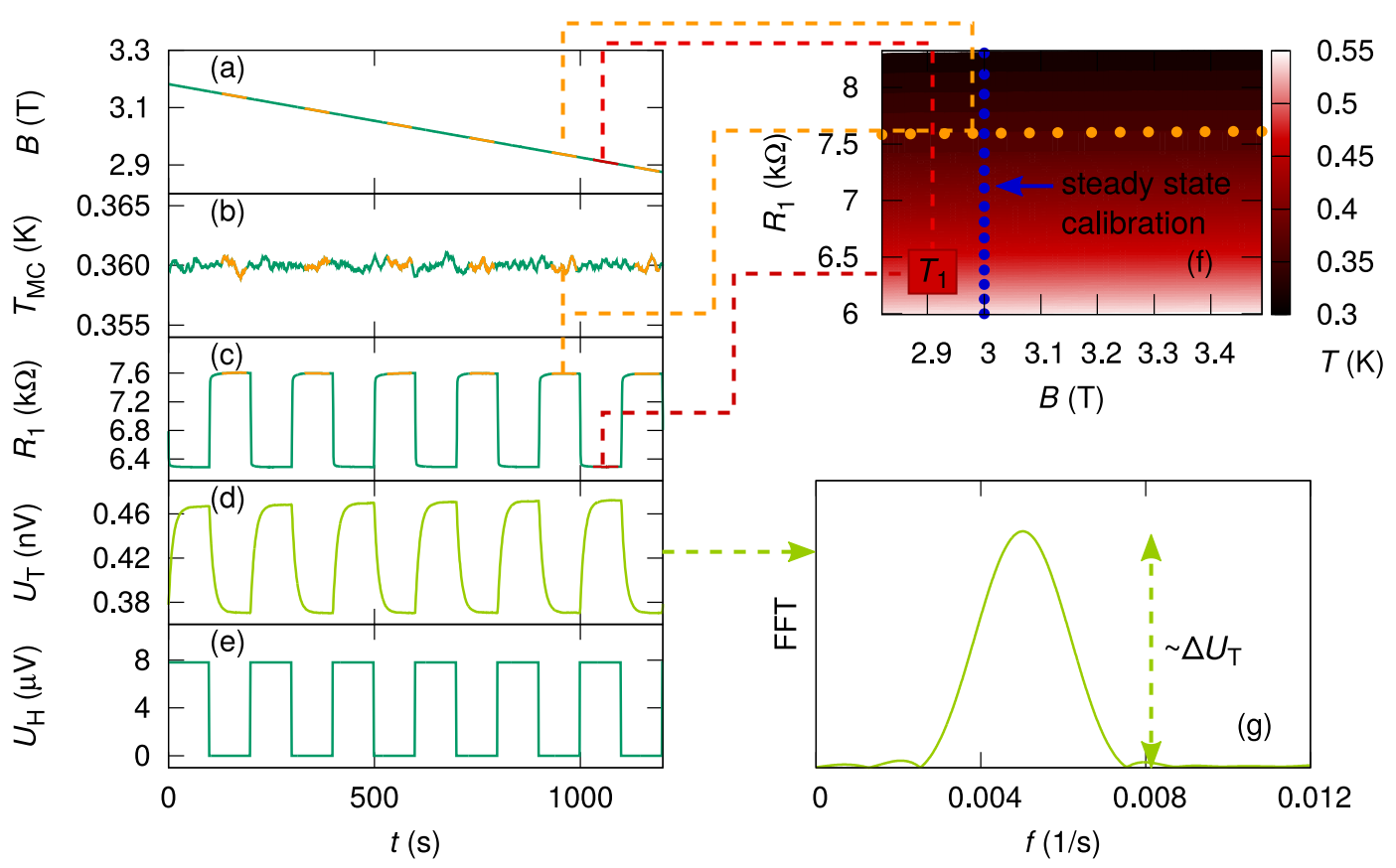

Figure 3.4: Measurement procedure for the pseudo-ac method. While the external parameter, in this case $B$, is swept (a), the heater is switched on and off (e). Four periods of the induced square wave in the thermovoltage $U_{\mathrm{T}}(\mathrm{d})$ produce a sharp peak in the fast Fourier transformation (FFT) spectrum (g). Its height is proportional to the thermovoltage drop $\Delta U_{\mathrm{T}}$ across the sample. The calibration map for the sample thermometer (f) is constructed from a standard steady-state calibration at constant field (blue dots) and from the heater-off points of $R_{1}(\mathrm{c}), T_{\mathrm{MC}}$ (b), and $B$ (a) (yellow dots). The points are linearly extrapolated across the $B-R_{1}$ plane.

by the calibrated thermometer. The external parameter is swept continuously, while the sample heater is alternately switched on and off. This creates an almost rectangular wave (depending on the coupling of the sample to the cold finger) of the sample thermometer resistances $R_{1,2}$, the voltage between the thermovoltage leads $U_{\mathrm{T}}$, the heater current $I_{H}$ and the heater voltage $U_{H}$. A Fourier analysis of $U_{\mathrm{T}}$ leads to the thermovoltage $\Delta U_{\mathrm{T}}$, the ones from heater current and heater voltage give $P_{\mathrm{H}}$. To deduce the sample temperatures from the sample thermometer resistances, one also needs a calibration $T\left(R_{1,2}, x\right)$. It is constructed from a) the resistance values of the $x$ sweep, when the sample heater is switched off, and from b) a separate $T\left(R_{1,2}\right)$ calibration for a fixed value of $x$. Since the changes of $R_{1}$ and $R_{2}$ stay small during the measurement, temperature variations of $R(x)$ in this interval are neglected and the points from a) are linearly extrapolated along the points from b). $\Delta T=T_{2}-T_{1}$ can then be extracted from the resistance values $R_{1,2}$ of the $x$ sweep, when the heater is switched on. A cross-check with an extensive calibration map for selected temperature windows verified a sufficient accuracy of this approximation.

If the thermal conductivity of the sample changes within the measured interval of $x$, the mean temperature of the sample will also change and the measurement is not truly 
isothermal. In cases where the deviations are too strong, the mixing-chamber temperature is, therefore, not left fixed, but is adjusted during the measurement using a feedback loop in the measurement program to ensure a constant average sample temperature in the heater-on state.

The resistivity can be determined in a similar fashion as described above. In this case, the electrical current $I_{\text {res }}$ is alternately switched on and off and the FFT of the $U_{\text {res }}$-signal determines the voltage drop $\Delta U_{\text {res }}$.

\subsection{Origin and Estimation of Measurement Uncertainties}

The Table 3.3 summarizes the estimated uncertainties for the sample temperature, the thermal conductivity, the thermopower and the resistivity. They result from the uncertainties of the different measured quantities, which are presented below. The values listed in the Table 3.3 are only a guide and do not represent true upper limits of the uncertainties.

\begin{tabular}{ccc}
\hline & systematic error & random error \\
\hline Sample temperature $T$ & $5 \%$ & $2 \%$ \\
Thermal conductivity & $13 \%$ & $3 \%$ \\
Thermopower & $5 \%$ & $2 \% \cdot S+0.01 \mu \mathrm{V} / \mathrm{K}$ \\
Resistivity & $8 \%$ & $1 \%$ \\
\hline
\end{tabular}

Table 3.3: Estimated uncertainties. They represent a guide for the reliability of the data presented in this thesis.

\section{Mixing-chamber temperature}

Using the PID control of the resistance bridge, the error of the mixing-chamber temperature is below $0.5 \%$ and, therefore, negligible.

\section{Sample temperature}

The measurement of the temperatures on the two contact points on the sample, $T_{1}$ and $T_{2}$, are most susceptible to errors. They determine the thermal gradient $\Delta T$ and the average sample temperature $T$. The uncertainty does not only depend on the error of the resistance measurement, which is below $2 \%$, but also on other factors.

The second error source for $T_{1}$ and $T_{2}$ comes from the calibration of the thermometers. In the case of the steady-state method, the errors of $T_{\mathrm{MC}}, R_{1}, R_{2}$, and of the linear interpolation sum up to a value of below $1 \%$. For the pseudo-ac method, the error for the linear interpolation across the whole $x-R_{1,2}$ calibration map is higher, but below $3 \%$.

The third error source is connected to small leakage currents of the current source for the sample heater. They lead to a systematic error of the calibration, which is performed at zero heater current. This error can be severe in the case of a very good thermal conductor where only a small gradient, hence small heater currents, can be applied for low-temperature 

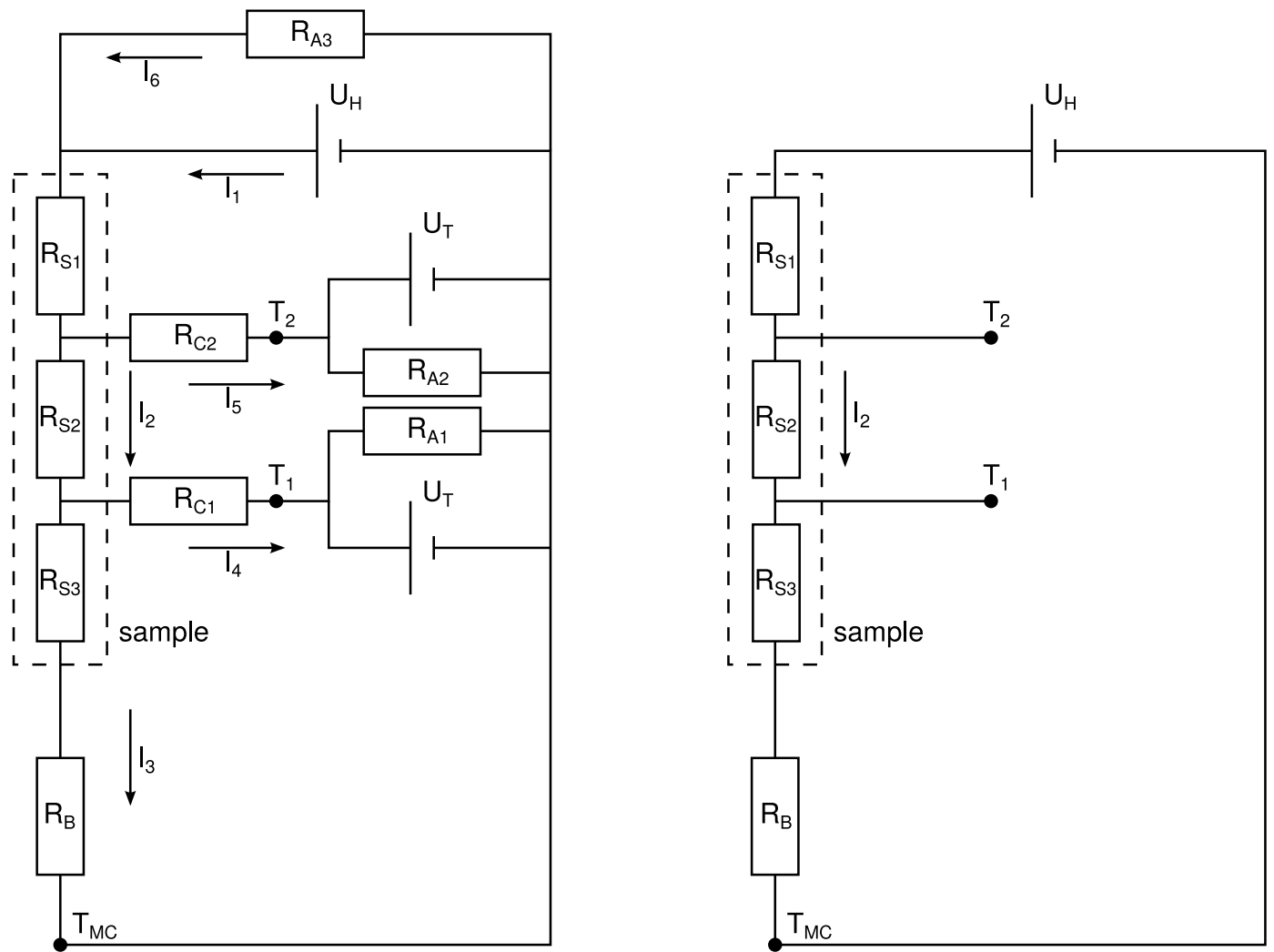

Figure 3.5: The real and the idealized electrical circuit representing the measurement of the thermal gradient.

measurements. Therefore, special care has to be taken for the electrical wiring of the current source.

The fourth error source derives from the relative thermal resistance between the thermometers and the sample, and between the thermometers and the environment. This must be sufficient to ensure that the thermal current is not bypassed through the electrical connections of the thermometers, the heater and the thermovoltage leads. For an estimation of the error of the thermal gradient $\Delta T$ due to a small heat leak, one can translate the thermal circuit of the set-up into the equivalent electrical circuit as shown in Figure 3.5. For simplicity, contributions from thermovoltage leads are neglected in the following.

The real gradient across the sample can be determined from the idealized circuit on the right side of Figure 3.5 to

$$
\Delta T_{\mathrm{r}}=I_{2} R_{\mathrm{S} 2}
$$

On the other hand, the measured gradient determined from the real circuit on the left side of Figure 3.5 is

$$
\Delta T_{\mathrm{m}}=I_{2} R_{\mathrm{S} 2}+I_{4} R_{\mathrm{C} 1}-I_{5} R_{\mathrm{C} 2}=\Delta T_{\mathrm{r}}+I_{4} R_{\mathrm{C} 1}-I_{5} R_{\mathrm{C} 2} .
$$


Using Kirchhoff's current laws, this expands to

$$
\frac{\Delta T_{\mathrm{m}}-\Delta T_{\mathrm{r}}}{I_{2}}=\frac{R_{\mathrm{C} 1}\left(R_{\mathrm{S} 3}+R_{\mathrm{B}}\right)\left(R_{\mathrm{A} 2}-R_{\mathrm{A} 1}\right)-R_{\mathrm{S} 2} R_{\mathrm{C} 2}\left(R_{\mathrm{C} 1}+R_{\mathrm{A} 1}+R_{\mathrm{S} 3}+R_{\mathrm{B}}\right)}{\left(R_{\mathrm{C} 2}+R_{\mathrm{A} 2}\right)\left(R_{\mathrm{C} 1}+R_{A 1}+R_{\mathrm{S} 3}+R_{\mathrm{B}}\right)} .
$$

Assuming a good link between the sample and the thermometers and a bad one between the thermometers and the environment, one can further simplify this equation using $R_{\mathrm{A} 1,2} \gg$ $R_{\mathrm{B}}, R_{\mathrm{C} 1,2} \gg R_{S 2,3}$ and Equation 3.4 .

$$
\frac{\Delta T_{\mathrm{m}}}{\Delta T_{\mathrm{r}}}=1+\frac{R_{\mathrm{B}} R_{\mathrm{C} 1}}{R_{\mathrm{S} 2}}\left(\frac{1}{R_{\mathrm{A} 1}}-\frac{1}{R_{\mathrm{A} 2}}\right)-\frac{R_{\mathrm{C} 2}}{R_{\mathrm{A} 2}}
$$

Some typical numbers for the resistances taken from Sey06a] are $R_{\mathrm{A} 1,2}=(30 \pm 1) \Omega, R_{\mathrm{C} 1,2}=$ $0.5 \Omega, R_{\mathrm{S} 2}=0.002 \Omega\left(\kappa=0.5 \mathrm{~W} \mathrm{~K}^{-2} \mathrm{~m}^{-1}, l_{\mathrm{S}} / A_{\mathrm{S}}=50000 \mathrm{~m}^{-1}\right), R_{\mathrm{B}}=0.1 \Omega$. They sum up to an error of

$$
\frac{\Delta T_{\mathrm{m}}}{\Delta T_{\mathrm{r}}}=1 \pm 0.02-0.02
$$

This quite large systematic error makes it necessary to take special care in the choice of the materials used for the wiring on the sample holder and the kind of contacts on the sample. One needs to ensure that $R_{\mathrm{A} 1,2} \gg R_{\mathrm{B}}, R_{\mathrm{C} 1,2} \gg R_{S 2,3}$.

In spite of a careful choice of the materials, one can observe a decoupling of the sample thermometers from the sample at low-enough temperatures. This is best visible in a bending over of the normally almost linear calibration curve $\log T\left(\log R_{1,2}\right)(c f$. Figure $3.3(\mathrm{~d}))$. This is usually caused by a heating of the thermometer due to an external heat entry, e.g. mechanical vibrations, and simultaneously a comparably weak link between sample and thermometer. Thus both, a better contact and a lower external heat entry can increase the measurable temperature window to lower $T$. Considerable work to improve the contacts is presented in Sey06a, Har10a. Those ideas were implemented in this work. Additionally, the mechanical damping of the whole set-up was improved as well as the filtering of the small signals and their shielding from electrical noise.

\section{Thermovoltage}

To avoid spurious thermovoltages between the thermovoltage leads and to minimize the background, the number of joints was minimized along the thermovoltage cables up to the voltmeter. The joints were placed inside the dilution refrigerator and thermally well anchored. Clamped joints were preferred over solder joints. The thermovoltage $\Delta U_{\mathrm{T}}$ is corrected for remaining spurious voltages between the thermovoltage cables by a background measurement in the heater off state. Systematic errors from wiring are, therefore, assumed to be negligible.

For the steady state, the random error of $\Delta U_{\mathrm{T}}$ calculated from the standard deviation amounts to $0.3 \mathrm{nV}$. Typical measured absolute values of $\Delta U_{\mathrm{T}}$ are $20 \mathrm{nV}$. They always stay below $0.5 \mu \mathrm{V}$ in our measurements. Therefore, the smallest relative error is below $1 \%$, but it becomes very large if the signal is small. The uncertainties of $\Delta U_{\mathrm{T}}$ determined by the pseudo-ac method can be attributed to the square-wave approximation and the accuracy of the fit on the FFT data. The resulting error amounts to roughly $0.05 \mathrm{nV}$. 


\section{Heater power}

The error of the heater power $P_{\mathrm{H}}$ stays below $1 \%$ and can be neglected.

\section{Resistivity}

The current source has an accuracy of far below 1\%. The accuracy of the voltage measurement also stays below $1 \%$.

\section{Sample dimensions}

The variation of the cross section $A_{\mathrm{S}}$ along the sample length and its measurement sum up to an accuracy of $3 \%$. The finite width of the contacts is responsible of an error of $5 \%$ for the length $l_{\mathrm{S}}$. Thus, the geometry factor $l_{\mathrm{S}} / A_{\mathrm{S}}$ enters with a systematic error of $8 \%$. Please note, that the systematic error due to the sample geometry in the thermal conductivity and the resistivity cancels out for the determination of the Lorenz ration $L / L_{0}=\rho \kappa / T$ if one measures $\rho$ and $\kappa$ on the same sample with the same contacts. 


\title{
4 \\ The Wiedemann-Franz Law in $\mathrm{YbRh}_{2} \mathrm{Si}_{2}$
}

\begin{abstract}
Based on Landau's description of metals within the Fermi-liquid theory and their phase transitions in terms of an order parameter, Hertz, Millis, and Moriya developed a successful theory of quantum phase transitions. Its predictions could nicely be confirmed on various Kondo-lattice systems such as $\mathrm{CeNi}_{2} \mathrm{Ge}_{2}$. However, a number of other heavy-fermion compounds were found, which do not fit into this pattern and require new theoretical models. In this chapter, we study the example of $\mathrm{YbRh}_{2} \mathrm{Si}_{2}$, which has a field-tuned unconventional quantum critical point (QCP). To investigate whether a theory for this QCP can be formulated in the framework of quasiparticles, we experimentally study the Wiedemann-Franz (WF) law as an established test for their existence. This law relates the electrical and thermal conductivity and is valid in the limit of zero temperature if quasiparticles exist. While we can confirm the WF law in the Fermi-liquid regime on either side of the QCP, we deduce a violation at the QCP from an extrapolation of our data. The extrapolation and the concluded violation of the WF law, however, is disputed due to an extra contribution in the thermal conductivity, which we ascribe to magnons and which masks the low-temperature behaviour. Our result puts strong constraints on any theory which tries to describe the QCP in $\mathrm{YbRh}_{2} \mathrm{Si}_{2}$. Most of the following work is published in $\mathrm{Pfa} 12 \mathrm{~b}$.
\end{abstract}

\subsection{Quantum Criticality in $\mathrm{YbRh}_{2} \mathrm{Si}_{2}$-Phenomenology}

$\mathrm{YbRh}_{2} \mathrm{Si}_{2}$ crystallizes in the tetragonal $\mathrm{ThCr}_{2} \mathrm{Si}_{2}$ structure with the space group $I 4 / \mathrm{mmm}$ (see Figure 4.1). The high-temperature effective moment of $\mu_{\mathrm{eff}}=4.4 \mu_{\mathrm{B}}$ is close to the one for an $\mathrm{Yb}^{3+}$ configuration Tro00]. The resulting $7 / 2$ multiplet is split into four Kramers doublets due to the crystal electric field [Sto06]. The ground-state doublet is well separated from the first excited level by $200 \mathrm{~K}$. $\mathrm{YbRh}_{2} \mathrm{Si}_{2}$ is a Kondo-lattice system. The energy scale corresponding to Kondo scattering on the whole $7 / 2$ multiplet is $80 \mathrm{~K}$ Köh08. At low temperatures, the scattering on the ground state dominates with an energy scale of $25 \mathrm{~K}$ Köh08, Geg06], which is also the scale where Kondo-lattice coherence develops Ern11. The Kondo effect leads to a screening of the magnetic moments towards $\mu_{\mathrm{eff}}=1.4 \mu_{\mathrm{B}}$ at low temperatures Geg02.

$\mathrm{YbRh}_{2} \mathrm{Si}_{2}$ develops an antiferromagnetic (AF) state below $T_{\mathrm{N}}=70 \mathrm{mK}$ Tro00 with a tiny ordered moment of the order of $10^{-3} \mu_{\mathrm{B}}$ Ish03. The order can be continuously suppressed towards a QCP by a very small magnetic field of $B_{\mathrm{c}}=0.06 \mathrm{~T}$ for $B \perp c$ and $B_{\mathrm{c}}=0.66 \mathrm{~T}$ for $B \| c$ Geg02, or by substitution of Si by Ge Cus03. In every case, the transition stays second order down to the lowest accessible temperatures [Kre09]. The studies presented in this chapter are performed for fields $B \perp c$, but are also compared to results for $B \| c$. 

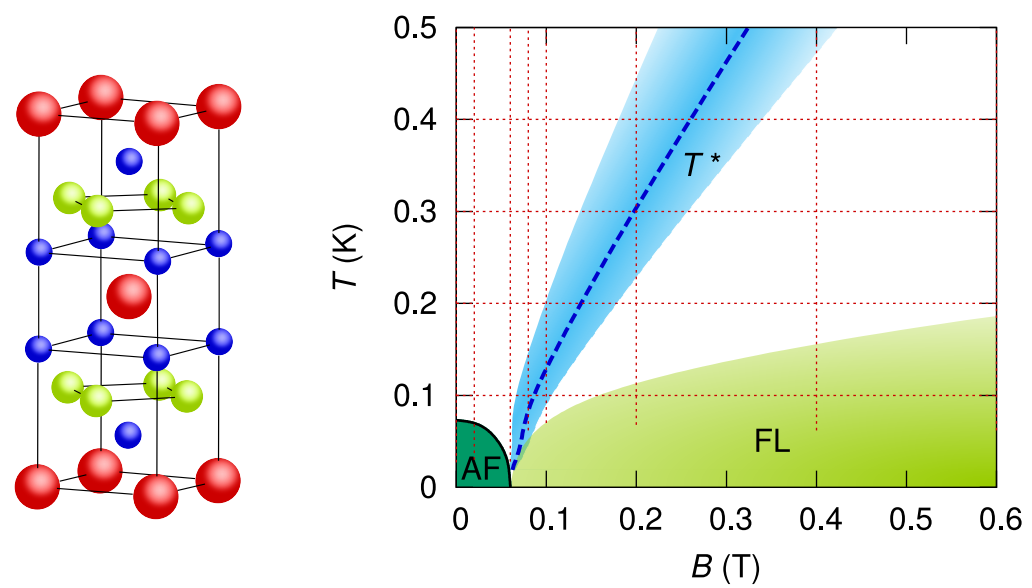

Figure 4.1: Left: Tetragonal crystal structure of $\mathrm{YbRh}_{2} \mathrm{Si}_{2}$ with $\mathrm{Yb}$ (red), $\mathrm{Rh}$ (green), and $\mathrm{Si}$ (blue). Right: $B-T$ phase diagram of $\mathrm{YbRh}_{2} \mathrm{Si}_{2}$ for $B \perp c$. The Néel temperature of the antiferromagnetic phase (AF) is suppressed towards a quantum critical point (QCP) by a tiny magnetic field of $60 \mathrm{mT}$. On the other side of the QCP, a paramagnetic Fermi liquid (FL) can be observed. The crossover scale $T^{*}$ terminates at the QCP and its width (FWHM) is proportional to $B$. The dashed vertical lines indicate the temperature scans of thermal and electrical transport measurements presented in this chapter. Horizontal lines are the field dependences derived from those $T$ scans.

Most of the signatures of quantum criticality are the same for both field directions and they are not distinguished in the following. However, there are certain qualitative differences, e.g. in the magnetoresistance Cus04. The QCP in Ge-substituted $\mathrm{YbRh}_{2} \mathrm{Si}_{2}$ shows similar properties to the one tuned by field, but it is not discussed in detail here.

The phase diagram around the magnetic-field-driven QCP is shown in Figure 4.1. A second phase transition, observed at $2.2 \mathrm{mK}$ in ultra-low-temperature measurements $\mathrm{Sch} 09 \mathrm{~b}$, is not included because its nature is still unclear. In the $\mathrm{AF}$ ordered phase for $B<B_{\mathrm{c}}$ and in the Fermi-liquid regime at $B>B_{\mathrm{c}}, \mathrm{YbRh}_{2} \mathrm{Si}_{2}$ shows typical signatures of a heavy Fermi liquid: the resistivity follows a $T^{2}$ dependence [Geg02]. The specific heat and the susceptibility are temperature independent Tro00, Geg02]. The large effective mass is confirmed by the large $A$ coefficient and a large $\gamma$ coefficient $\left(1.7 \mathrm{~J} / \mathrm{K}^{2}\right.$ for $\left.B=0\right)$ Geg02.

So far, the detailed structure of the AF phase could not be determined due to the tiny ordered moment and the low ordering temperature. However, despite the AF order at low $T$, a competition of both AF and ferromagnetic (FM) fluctuations was detected Ish02, Geg05, Sto12a.

In the quantum critical region and at the critical field, the physical properties show pronounced non-Fermi-liquid behaviour. Some of these properties are listed in Table 4.1 and are compared to the predictions from the SDW scenario. The dimensionality of the fluctuations is not known yet, but the linear resistivity and the logarithmic divergence of the specific heat $C / T$ Tro00 hints towards a 2D SDW picture at first glance. However, there are a number of deviations:

- The Grüneisen ratio $\Gamma(T)$ diverges with a power law Tok09 Küc03. The power 


\begin{tabular}{lllll}
\hline & & $\rho$ & $C / T$ & $\Gamma$ \\
\hline $\mathrm{YbRh}_{2} \mathrm{Si}_{2}$ & $(T>0.3 \mathrm{~K})$ & $T\left(\right.$ or $\left.T^{0.75}\right)$ & $\log (1 / T)$ & $T^{-2}$ \\
& $(T<0.3 \mathrm{~K})$ & $T\left(\right.$ or $\left.T^{0.75}\right)$ & $T^{-0.43}$ & $T^{-0.7}$ \\
$\mathrm{SDW}$ & $2 \mathrm{D} \mathrm{AF}$ & $T$ & $\log (1 / T)$ & $\frac{\log \log (1 / T)}{T \log (1 / T)}$ \\
& 3D AF & $T^{3 / 2}$ & $-T^{1 / 2}$ & $-T^{-1}$ \\
& 2D FM & $T^{4 / 3}$ & $T^{-1 / 3}$ & $T^{-2 / 3} \log (1 / T)$ \\
& 3D FM & $T^{5 / 3}$ & $\log (1 / T)$ & $\left(T^{2 / 3} \log (1 / T)\right)^{-1}$ \\
\hline
\end{tabular}

Table 4.1: Comparison of the spin-density wave (SDW) scenario (Hertz-Millis-Moriya theory) with the properties observed in $\mathrm{YbRh}_{2} \mathrm{Si}_{2}$ at $B_{\mathrm{c}}$. The predictions for the SDW-type QCP for the resistivity $\rho$ and for the specific heat $C / T$ are taken from [Ste01], for the Grüneisen ratio $\Gamma$ from Zhu03. See text for the results on $\mathrm{YbRh}_{2} \mathrm{Si}_{2}$. The resistivity dependence is indicated for samples with a low (high) residual resistivity ratio (RRR).

changes at a crossover scale of $0.3 \mathrm{~K}$.

- The specific heat turns from a logarithmic $T$ dependence towards a power-law divergence below the same scale of $0.3 \mathrm{~K}$ Oes08, Tro00, Küc03.

- The effective mass diverges at the QCP as $m^{*} \propto\left(B-B_{\mathrm{c}}\right)^{-0.33}$, which was observed in $\mathrm{YbRh}_{2}\left(\mathrm{Si}_{0.95} \mathrm{Ge}_{0.05}\right)_{2}$ Cus03.

- For samples with a low RRR, the resistivity follows a linear temperature dependence up to $1 \mathrm{~K}$ in $\mathrm{YbRh}_{2} \mathrm{Si}_{2}$ [Tro00] and up to $10 \mathrm{~K}$ in $\mathrm{YbRh}_{2}(\mathrm{Si}, \mathrm{Ge})_{2}$ [Cus03.

- The linear-in- $T$ regime of the resistivity decreases with increasing sample quality. Highquality single crystals with $\rho_{0} \approx 0.5 \mu \Omega \mathrm{cm}$ and a RRR of 150 can be described by a linear resistivity only below $150 \mathrm{mK}$. New analyses show, that in a wide $T$ range they follow $\rho=\rho_{0}^{\prime}+A^{\prime} T^{0.75}$ Geg08, Ste14, Wöl11.

- The SDW scenario for a $2 \mathrm{D}$ AF would lead to $T_{\mathrm{N}} \propto\left|B-B_{\mathrm{c}}\right|$, which is not the case for $\mathrm{YbRh}_{2} \mathrm{Si}_{2}$.

Several thermodynamic and transport measurements revealed an additional energy scale $T^{*}$ in $\mathrm{YbRh}_{2} \mathrm{Si}_{2}$ Pas04, Geg07, which is also shown in the phase diagram in Figure 4.1. It terminates at the QCP [Fri10], but can be detached from it by chemically applied pressure Fri09. The experimentally observed signatures are crossovers. The width of them, however, was shown to extrapolate linearly towards zero for $T \rightarrow 0$ in case of the resistivity $\rho(B)$ and the Hall coefficient $R_{\mathrm{H}}(B)$ Fri10. The abrupt jump in $R_{\mathrm{H}}(B)$ at $T=0$ was interpreted as a reconstruction of the Fermi surface Pas04, which takes place at $B_{\mathrm{c}}$ in the pure $\mathrm{YbRh}_{2} \mathrm{Si}_{2}$.

The unusual non-Fermi-liquid behaviour in the quantum critical regime and the properties connected to the energy scale $T^{*}$ are beyond the predictions of the SDW scenario and new theoretical concepts are required.

\subsection{Quantum Criticality in $\mathrm{YbRh}_{2} \mathrm{Si}_{2}$-Theoretical Concepts}

The experimental results on $\mathrm{YbRh}_{2} \mathrm{Si}_{2}$ at the QCP, especially the diverging effective mass and the unusual power laws, are incompatible with the SDW scenario. Earlier on, another 

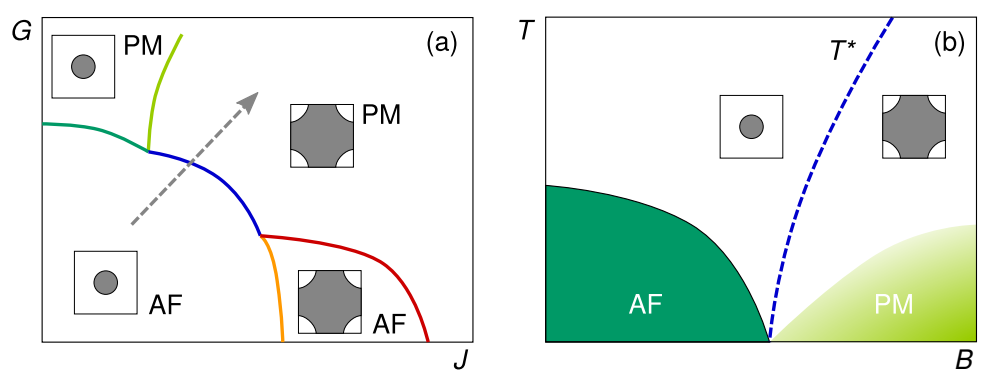

Figure 4.2: The global phase diagram. (a) The global phase diagram spanned by the fluctuations of the local moment $G$ and the Kondo coupling $J$ offers several paths to change from an antiferromagnetically ordered state (AF) to a paramagnetic one (PM). The paths differ in the position of the Fermi surface change. The large (small) Fermi-surface indicates that the $f$ electrons are (not) incorporated. $\mathrm{YbRh}_{2} \mathrm{Si}_{2}$ seems to follow the path denoted by the arrow in (a) and depicted in detail in (b). Here, the Fermi-surface reconstruction coincides with the onset of magnetic order. After Si11.

unconventional QCP was found in $\mathrm{CeCu}_{6-x} \mathrm{Au}_{x}$, which also shows unconventional behaviour and signs of local criticality Löh07; Sto11. These discoveries stimulated the development of new theoretical approaches beyond Hertz-Millis-Moriya in the last few years. The nature of the QCP in $\mathrm{YbRh}_{2} \mathrm{Si}_{2}$, however, is still highly debated.

\subsubsection{The Kondo-Breakdown Scenario}

Si et al. Si01 and Coleman et al. Col01 proposed an alternative scenario for $\mathrm{CeCu}_{6-x} \mathrm{Au}_{x}$, which was later also applied to $\mathrm{YbRh}_{2} \mathrm{Si}_{2}$. They suggested that the heavy composite quasiparticles in a Kondo system may break up and disintegrate into local $f$ electrons and conduction electrons above an energy scale $E^{*}$. This scale corresponds to the observed $T^{*}$ and results in a QCP as $T \rightarrow 0$. Consequently, the whole Fermi surface reconstructs at $E^{*}$ from one, which incorporates the $f$ electrons, to another, which does not. As an experimental signature, the Hall coefficient jumps at the QCP. The critical fluctuations may be thought of as fluctuations between these two Fermi surfaces. Hence, they are fermionic in nature and critical on the whole Fermi surface in contrast to the bosonic fluctuations with a certain wave vector $Q$ for an AF SDW QCP. As a consequence, the picture of Landau quasiparticles is no longer valid at the Kondo-breakdown QCP.

In the case of pure $\mathrm{YbRh}_{2} \mathrm{Si}_{2}$, the order instantaneously sets in at the QCP of the Fermisurface reconstruction. This seems accidental in the light of the "global phase diagram" developed later and also in view of the experiments on Co and Ir substitution series Fri09, which fit into this phase diagram (Figure 4.2 ). The localized $f$ moments above $E^{*}$ are responsible for the $\mathrm{AF}$ ordering in the pure $\mathrm{YbRh}_{2} \mathrm{Si}_{2}$. The high effective mass in this ordered phase, which was observed in $\mathrm{YbRh}_{2} \mathrm{Si}_{2}$, is due to the dynamical Kondo effect (see Si13 and references therein). 


\subsubsection{The Lifshitz Scenario}

Hackl and Vojta Hac11] introduced the idea that a Zeeman-driven Lifshitz transition is responsible for the $T^{*}$ line. The band structure of their model contains a Fermi-surface pocket at the right distance from the Fermi energy $\epsilon_{\mathrm{F}}$. It is split by a magnetic field and moves through $\epsilon_{\mathrm{F}}$ at the field $B^{*}=B\left(T^{*}\right)$. The Lifshitz scenario predicts a scale $T^{*}$, at which thermodynamic and transport properties, especially the Hall effect, show distinct crossovers as a function of field. They become sharp but continuous for $T=0$. Apparent non-Fermiliquid behaviour occurs in the temperature dependences of thermodynamic quantities around this transition, but the system stays a Fermi liquid at all fields in the low-temperature limit. However, the model does not capture any magnetic ordering nor does it account for strong correlations Fri13 Hac13. Hence, experimental observations such as the divergent effective mass at $B_{\mathrm{c}}$ cannot be reproduced.

\subsubsection{Critical Quasiparticle Theory}

The phenomenological critical quasiparticle theory introduced in Wöl11; Abr12, Abr14 describes the AF QCP in $\mathrm{YbRh}_{2} \mathrm{Si}_{2}$ within two stages, which are separated by the crossover scale of $0.3 \mathrm{~K}$. In the first regime above this temperature, the critical fluctuations are Gaussian, i.e.non-interacting, and either 2D AF fluctuations or 3D FM fluctuations. The heavy composite quasiparticles interact with these Gaussian fluctuations leading to a logarithmic mass enhancement. Below $0.3 \mathrm{~K}$, the critical fluctuations change to weakly interacting 3D AF fluctuations. This also influences the coupling of the fluctuations to the quasiparticles. If the mass enhancement in the first regime is strong enough, the system is driven to a strong-coupling scenario which can lead to a power-law divergence of $m^{*}$. For the other case - the weak-coupling scenario - the system would behave like for a typical SDW QCP described by Hertz, Millis, and Moriya.

The induced order is of an itinerant-heavy-quasiparticle SDW type with a small ordered moment. The critical AF fluctuations with the wave vector $Q$ would lead to limited regions of the Fermi surface, which become "hot". A diverging mass on the whole Fermi surface is either induced by impurity scattering, which smears these hot regions [Wöl11; Abr12], or by a combined exchange of two AF fluctuations [Abr14.

The theory is able to predict the exponents for the power-law behaviour of many observables in accordance with experimental results. An important drawback with respect to the phase diagram of $\mathrm{YbRh}_{2} \mathrm{Si}_{2}$ is the additional energy scale $T^{*}$, which is not captured by the critical quasiparticle theory. Hence, there is no destruction of the Kondo effect at the QCP in this model. In contrast to the Kondo-breakdown scenario, the heavy quasiparticles in the critical quasiparticle theory are preserved in the whole phase diagram. This implies that the properties in the non-Fermi-liquid region can be described by Landau quasiparticles.

\subsection{Motivation}

Many experiments were already performed on $\mathrm{YbRh}_{2} \mathrm{Si}_{2}$ to study the properties of the QCP and to establish constraints on any theoretical model which tries to explain its unconventional nature. The aim of our study is to determine whether a theory can be formulated in a Landau-quasiparticle picture, or whether this picture breaks down at the QCP. In two of 
the three theories presented above, namely the Lifshitz scenario and the critical quasiparticle scenario, quasiparticles stay intact at the QCP in contrast to the Kondo-breakdown scenario.

To test the fate of Landau quasiparticles, the Wiedemann-Franz (WF) law is an ideal tool. It relates the electrical and thermal conductivity. If quasiparticles are well defined, their ratio $L=\kappa / T \sigma$ is a fundamental physical constant $\mathrm{L}_{0}=\left(\pi k_{\mathrm{B}}\right)^{2} / 3 e^{2}$ at $T=0$ (see also Section 2.7.3. Deviations from $L / \mathrm{L}_{0}=1$ can be categorized into three cases:

- $L / \mathrm{L}_{0}=0$ : superconducting state

- $L / \mathrm{L}_{0}>1$ : other heat carriers in addition to electronic quasiparticles

- $L / \mathrm{L}_{0}<1$ : sign of inelastic scattering

If $L / \mathrm{L}_{0}<1$ persists down to $T=0$, the WF law is violated and the quasiparticle picture breaks down.

In a theoretical treatment, Mahajan et al. Mah13 pointed out that this simple view, which describes the violation of the WF law as a perturbation of the Fermi-liquid ground state introducing additional inelastic scattering, is insufficient when quasiparticles do not exist at all. From their perspective, the Lorenz number is not a reference point any more. Nevertheless, an experimental result of $L / \mathrm{L}_{0}<1$ requires a theory beyond Landau quasiparticles.

A reliable extrapolation to zero temperature is essential to test the WF law. Therefore, we put special effort into very-low-temperature measurements and into a clear concept for the extrapolation of these results to $T=0$. A fixed point in this concept is the high-field phase, which is a Fermi liquid without any doubt. To confirm the WF law in this regime is an important starting point. In the next step, the WF law is tested in the AF phase, which is also a Fermi liquid. This is challenging due to the low ordering temperature. In the third step, we compare those results with the behaviour at the critical field.

\subsection{Experimental Methods}

Both samples used for the study of the WF law were cut from the same crystal of batch \#63129. Thermal and electrical transport coefficients were obtained from the same rectangular-shaped $\left(4.2 \times 0.5 \times 0.1 \mathrm{~mm}^{3}\right)$ single crystal (sample 1) with identical contact geometry. This allows a reliable determination of the Lorenz ratio $L(T) / \mathrm{L}_{0}=\rho(T) / w(T)$, since the geometry factor $l_{\mathrm{S}} / A_{\mathrm{S}}$ cancels out. $l_{\mathrm{S}}$ and $A_{\mathrm{S}}$ are the contact distance and the crosssection of the sample, respectively. Additional measurements of the electrical resistivity were performed on a second single crystal (sample 2) with a different geometry factor $(1.7 \times$ $\left.0.41 \times 0.06 \mathrm{~mm}^{3}\right)$. Because of the inhomogeneous thickness of the samples, the geometry factor could not be determined very precisely. However, the measured resistivities of sample 2 could perfectly be rescaled to those of sample 1 by a factor $1.25 \pm 0.03$ and a correction for the residual resistivity of $0.22 \mu \Omega \mathrm{cm}$.

Heat and charge currents as well as the magnetic field were applied within the basal tetragonal plane. However, we did not consider the distinction between the [100] and the [110] directions. The parallel orientation of the magnetic field to the heat and charge flow allows us to neglect the contributions of transverse effects (Nernst and electrical/thermal Hall effects) in all measurements. 


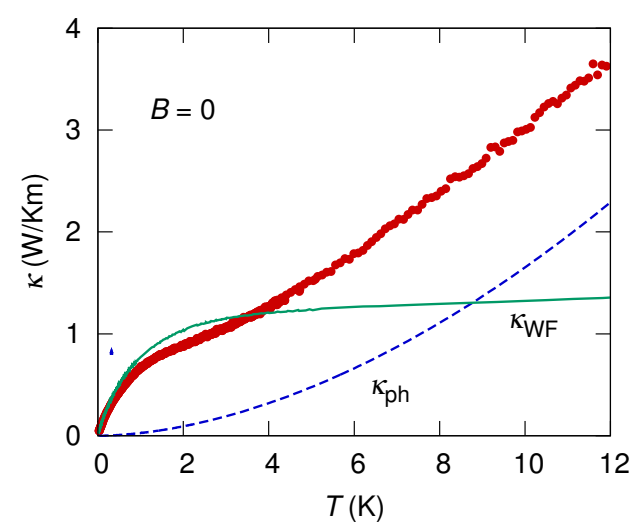

Figure 4.3: Estimation of the phonon contribution to the thermal conductivity. The total thermal conductivity $\kappa(T)$ (red dots) at zero field is shown up to $12 \mathrm{~K}$. For comparison, we calculated $\kappa_{\mathrm{WF}}(T)=\mathrm{L}_{0} T / \rho(T)$ as an indication of the electronic contribution. To determine the phonon contribution, we subtract $\kappa_{\mathrm{WF}}(T)$ from $\kappa(T)$ above $6 \mathrm{~K}$. A power-law fit of the result is extrapolated to lower temperatures and serves as an estimate for $\kappa_{\mathrm{ph}}(T)$.

The thermal conductivity was measured using the "Franz" set-up with the standard twothermometers-one-heater steady-state method. The raw data of the thermal conductivity $\kappa$ were averaged and the error bars in the following plots represent the standard deviation. Systematic errors would only shift the curves. Due to the very low Néel temperature $T_{\mathrm{N}}=$ $70 \mathrm{mK}$, considerable effort has been made to extend the thermal-conductivity measurements down to $25 \mathrm{mK}$ at $B=0$ and $0.02 \mathrm{~T}$ compared to previous studies, which reached only $40 \mathrm{mK}$ (Ref. [Tom11]) and $60 \mathrm{mK}$ (Ref. Tan07]), respectively. To reduce the statistical error especially at such low temperatures, substantially more temperature scans have been performed at $B=0$. At the critical field, $30 \mathrm{mK}$ has been reached as the lowest temperature; $60 \mathrm{mK}$ for all other fields.

The electrical resistivity $\rho(T)$ was determined by a four-point $\mathrm{AC}$ technique in a ${ }^{3} \mathrm{He}-{ }^{4} \mathrm{He}$ dilution refrigerator down to $T \approx 20 \mathrm{mK}$ in the AF phase, $40 \mathrm{mK}$ at $B_{\mathrm{c}}$ due to heating effects, and $30 \mathrm{mK}$ above $B_{\mathrm{c}}$.

\subsection{Thermal and Electrical Transport}

\subsubsection{Phonon Contribution}

The thermal conductivity $\kappa(T)$ between $25 \mathrm{mK}$ and $12 \mathrm{~K}$ is shown in Figure 4.3 for $B=0$. For comparison, $\kappa_{\mathrm{WF}}(T)=\mathrm{L}_{0} T / \rho(T)$ was calculated from the measured electrical resistivity $\rho(T)$. Above $4 \mathrm{~K}, \kappa(T)$ exceeds $\kappa_{\mathrm{WF}}(T)$ due to the contribution of phonons $\kappa_{\mathrm{ph}}(T)$ to the heat transport. Below $4 \mathrm{~K}, \kappa_{\mathrm{ph}}(T)$ is suppressed, and $\kappa(T)$ becomes smaller than $\kappa_{\mathrm{WF}}(T)$. The crossing indicates the presence of inelastic scattering processes. The contribution of phonons can be estimated by $\kappa_{\mathrm{ph}}(T)=\kappa(T)-\kappa_{\mathrm{WF}}(T)$ above $6 \mathrm{~K}$. The result is found to follow a $T^{\epsilon}$ dependence with $\epsilon=2 \pm 0.2$. An extrapolation of the power law to lower temperatures serves as an estimation of the phonon contribution and indicates a negligible $\kappa_{\mathrm{ph}}(T)$ below $1 \mathrm{~K}$, i.e., within the temperature range of interest in the present work. Later we will see, that $\kappa(T)$ becomes again somewhat larger than $\kappa_{\mathrm{WF}}(T)$ below $35 \mathrm{mK}$ due to a magnon contribution (Figure 4.4(a)). 


\subsubsection{Low-Temperature Data and the Wiedemann-Franz Law}

Figure 4.4 depicts the low-temperature behaviour of both the electrical resistivity $\rho(T)$ and the thermal resistivity $w(T)=\mathrm{L}_{0} T / \kappa(T)$ in the AF phase $(B=0,0.02 \mathrm{~T})$, at the critical field $\left(B=0.06 \mathrm{~T} \approx B_{c}\right)$, and in the paramagnetic Fermi-liquid region $\left(B>B_{c}\right)$. For a direct comparison, $w(T)$ is shown in the same units as $\rho(T)$. Figure 4.5 displays the difference $\delta(T)=w(T)-\rho(T)$ and the Lorenz ratio $L / \mathrm{L}_{0}$ for the data shown in Figure 4.4. These direct comparisons show that $w(T)$ exceeds $\rho(T)$ over a wide range of temperature and field indicating strong inelastic scattering.

\section{Paramagnetic Fermi-Liquid Regime}

Below $T=0.15 \mathrm{~K}$ and for $B \geq 0.6 \mathrm{~T}, w(T)=\rho(T)$ within the experimental resolution. This is illustrated in Figure 4.5(f), which shows that $\delta(T)$ approaches zero, and in Figure 4.5(c), which demonstrates that $L(T) / \mathrm{L}_{0}=1$. In this high-field range, both $\Delta \rho(T)=\left[\rho(T)-\rho_{0}\right] \propto$ $T^{2}$ and $\left[w(T)-w_{0}\right] \propto T^{2}$ below the Fermi-liquid crossover temperature. Here, $\rho_{0}$ and $w_{0}$ are the residual electrical and thermal resistivities. These results establish the validity of the WF law in the Fermi-liquid phase for $B \geq 0.6 \mathrm{~T}$. For $0.2 \mathrm{~T}$ and $0.4 \mathrm{~T}$, the results shown in Figure 4.5 suggest similar Fermi-liquid behaviour at lower temperatures.

\section{Antiferromagnetic Phase and Magnon Contribution}

Upon cooling in zero field and for $0.02 \mathrm{~T}, \rho(T)$ drops at the Néel transition into the AF phase, reflecting the freezing out of spin-disorder scattering. Below $T_{\mathrm{N}}, \rho(T)$ exhibits a Fermi-liquid $T^{2}$ dependence ( $c f$. Ref. Geg02]). $w(T)$ drops as well, but already at higher temperature $(T \approx 0.1 \mathrm{~K}$ at $B=0)$. Furthermore, $\delta(T)$ becomes negative below $T=33 \mathrm{mK}$ (Figure 4.5(d)), and $L / \mathrm{L}_{0}$ exceeds one (Figure 4.5(a)). An upturn of $L(T) / \mathrm{L}_{0}$ just below $T_{\mathrm{N}}$ was also observed in Ref. Tom11] down to $50 \mathrm{mK}$, where $L(T) / \mathrm{L}_{0}$ is still below one but appears to extrapolate to one as $T \rightarrow 0$. Recent studies also show the same upturn Mac13; Rei14; Pou14 and $L(T) / \mathrm{L}_{0}>1$ is observed in the cases where low-enough temperatures were reached [Mac13; Pou14] ( $c f$. Figure 4.9).

$w(T)$ may drop because inelastic scattering of electronic quasiparticles off spin fluctuations freezes out. However, the observation of $L(T) / \mathrm{L}_{0}>1$ below $T=0.033 \mathrm{~K}$ clearly shows that the thermal transport is not entirely due to electronic quasiparticle transport, as concluded in Ref. [Tom11]. Instead, it is masked by that of an additional heat channel $\kappa_{\mathrm{m}}(T)$. Its contribution falls in the range $2-5 \cdot 10^{-3} \mathrm{~W} / \mathrm{Km}$ between 25 and $30 \mathrm{mK}$. Because this value of $\kappa_{\mathrm{m}}(T)$ is close to the experimental uncertainty, its detailed temperature dependence cannot be determined. Nevertheless, its existence has been confirmed by repeated measurements and by recent studies Mac13, Pou14. Lattice vibrations are unsuitable to account for this extra thermal conductivity: The largest $\kappa_{\mathrm{ph}}$ is estimated to be less than $1 \cdot 10^{-5} \mathrm{~W} / \mathrm{Km}$ in this range using the lattice specific heat Cus03, and the sample dimension $(\approx 100 \mu \mathrm{m})$ limiting the mean free path.

Another potential thermal-transport channel is that of AF magnons. Signatures of spinwave excitations were observed in the zero-field specific heat examined down to $T=18 \mathrm{mK}$ for an $\mathrm{YbRh}_{2} \mathrm{Si}_{2}$ single crystal being of similar quality as samples 1 and 2 Cus03. One can roughly estimate the magnitude of the magnon thermal conductivity with the classical kinetic relation, $\kappa_{\mathrm{m}}=(1 / 3) C_{\mathrm{m}} v_{\mathrm{m}} l_{\mathrm{m}}$, where $l_{\mathrm{m}}$ is the magnon mean free path, $C_{\mathrm{m}}$ and $v_{\mathrm{m}}$ are the magnon specific heat and velocity. To obtain $C_{\mathrm{m}}$ and $v_{\mathrm{m}}$, one can analyse the specific 


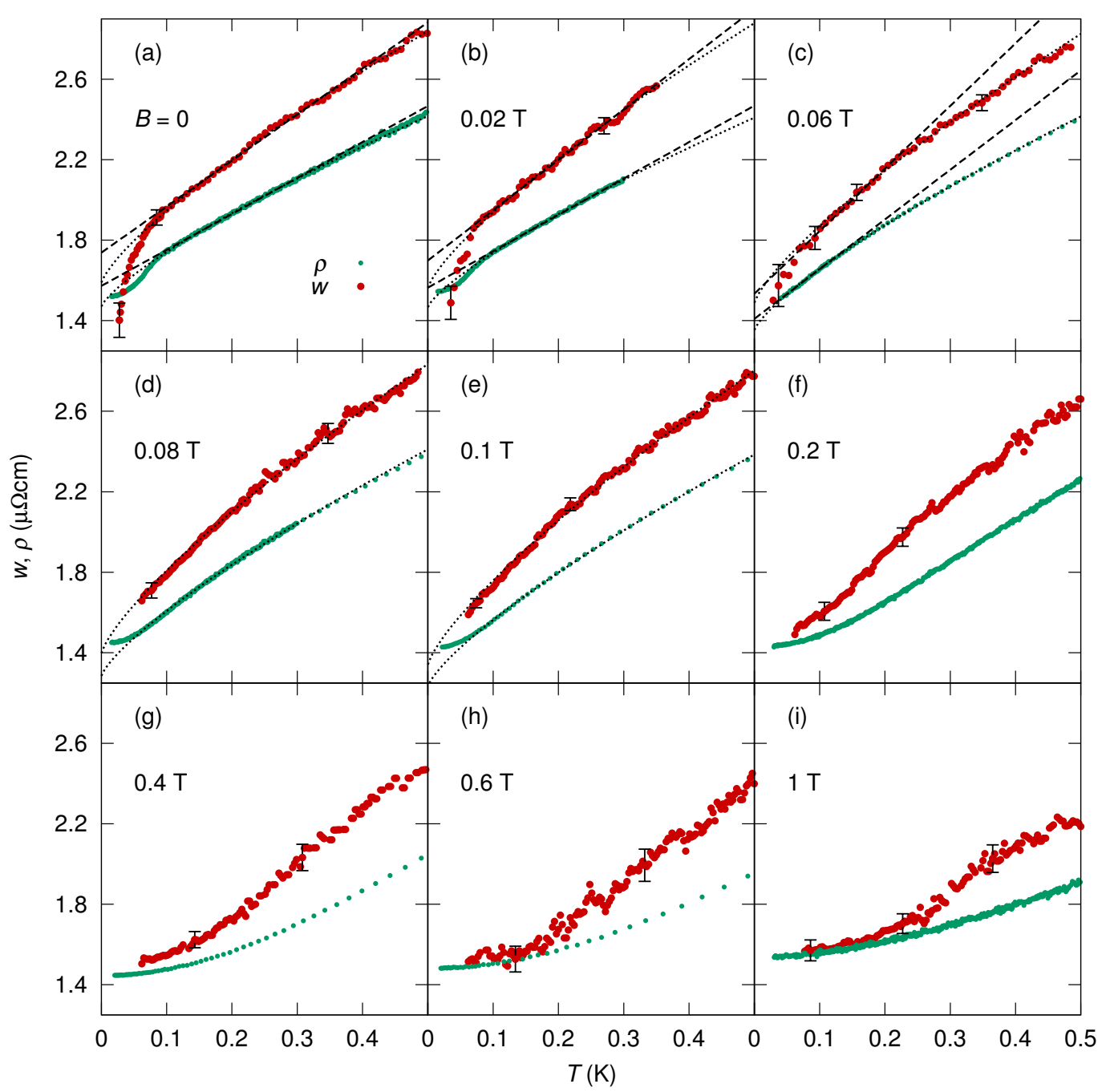

Figure 4.4: Thermal and electrical resistivity. The thermal resistivity $w(T)=\mathrm{L}_{0} T / \kappa(T)$ (red) and the electrical resistivity $\rho(T)$ (green) are shown below $T=0.5 \mathrm{~K}$ and at different fields between $B=0$ and $1 \mathrm{~T}$. The error bars show representative standard deviations. The lines correspond to extrapolations of the data in the quantum critical regime, which is indicated in Figure 4.7 Long dashed lines are linear fits, short dashed lines are fits with a power law $\rho_{0}^{\prime}+A^{\prime} T^{0.78}$. The power 0.78 is deduced from a prior fit to $\rho(T, B=0.06 \mathrm{~T})$. The fit range for all curves starts above $T_{\mathrm{N}}$ and $T_{\mathrm{FL}}$ for $\rho(T)$ and above the onset of the magnon contribution for $w(T)$ (see main text). The fit interval for the linear fits at $B=0,0.02 \mathrm{~T}$, and $0.06 \mathrm{~T}$ ends at $0.35 \mathrm{~K}, 0.30 \mathrm{~K}$, and $0.12 \mathrm{~K}$ for $\rho$, respectively, and at $0.40 \mathrm{~K}, 0.35 \mathrm{~K}$, and $0.2 \mathrm{~K}$ for $w$. For the power law fit, it ends at $0.5 \mathrm{~K}$ for all curves. 

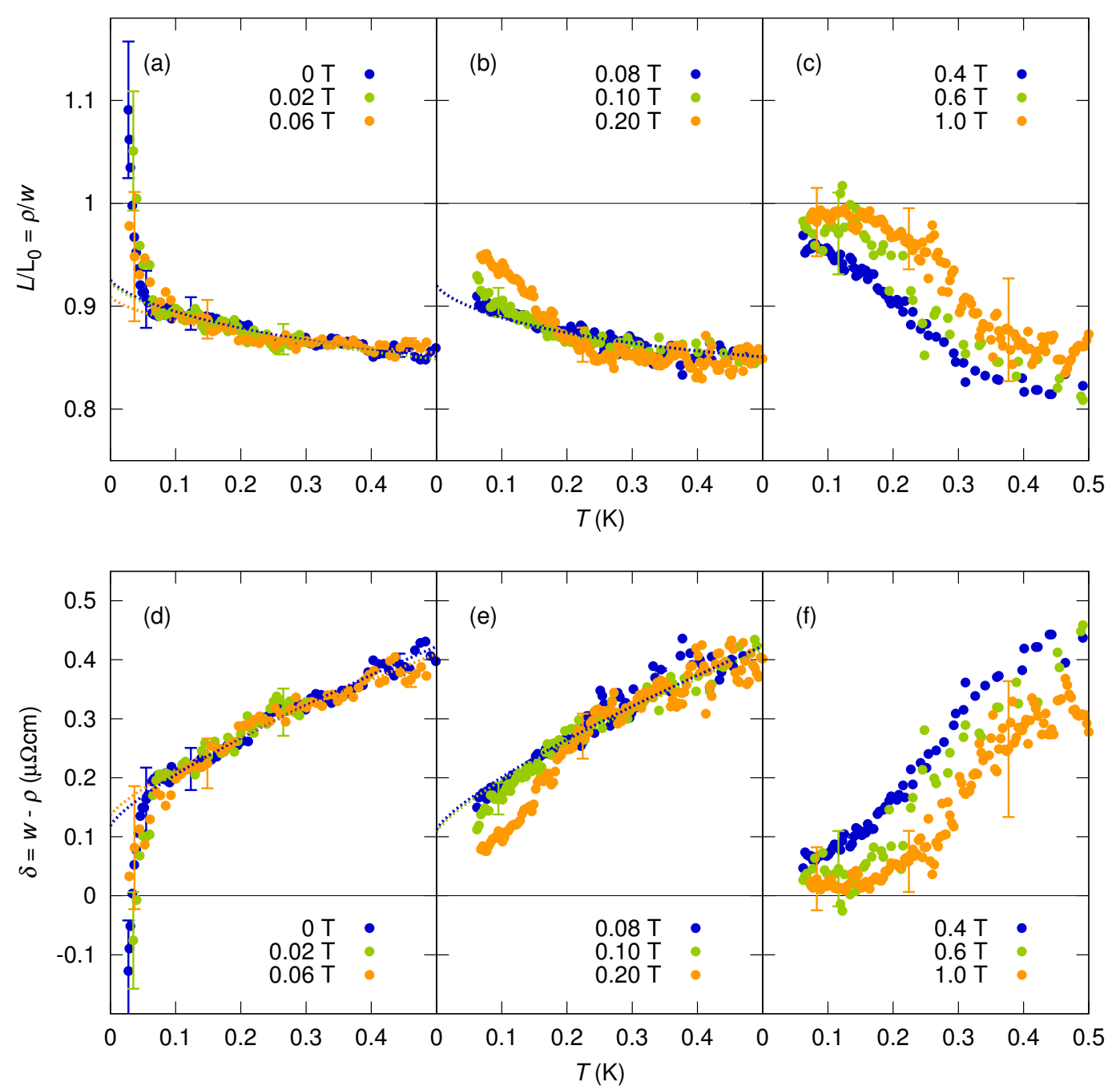

Figure 4.5: Validity and violation of the WF law. The temperature dependence of the Lorenz ratio $L / \mathrm{L}_{0}=\rho / w((\mathrm{a})-(\mathrm{c}))$ and of the difference $\delta=\rho-w((\mathrm{~d})-(\mathrm{f}))$ is shown for the same fields as in Figure 4.4 The error bars are derived from the standard deviation in the data of Figure 4.4. The WF law is found to be valid below $T=0.15 \mathrm{~K}$ for $B=1 \mathrm{~T}$ and $0.6 \mathrm{~T}((\mathrm{c}),(\mathrm{f}))$. It is anticipated to hold also at lower $T$ and $B \geq 0.2 \mathrm{~T}$ ((b),(c),(e),(f)). The dashed lines in (a),(b),(d),(e) correspond to power law fits $a+b T^{0.78}$ to $w(T)$ and $\rho(T)$ in the quantum critical regime as shown in Figure 4.4 Their $T=0$ extrapolation demonstrates a violation of the WF law by approximately $10 \%$ in a putative paramagnetic non-Fermi-liquid ground state. (a),(d) The strong deviations from the fits at low $T$ for $B \leq 0.06 \mathrm{~T}$ and a Lorenz ratio $L / \mathrm{L}_{0}>1$ illustrates the onset of a magnon contribution to the thermal transport, which is supposed to vanish for $T \rightarrow 0$. 


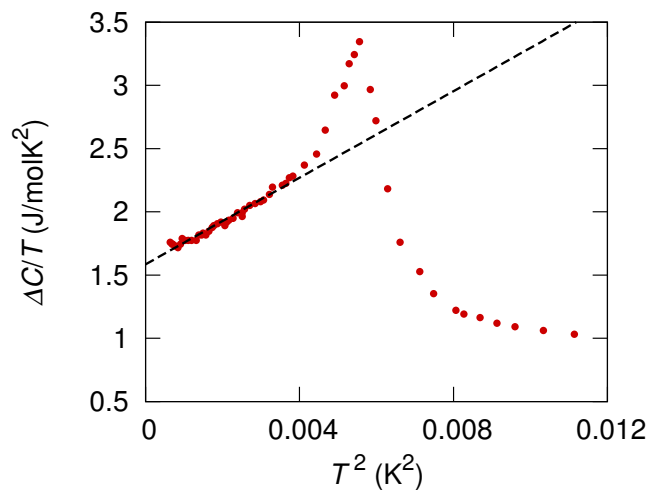

Figure 4.6: Specific heat of $\mathrm{YbRh}_{2} \mathrm{Si}_{2}$ at $B=$ 0 as $\Delta C / T$ versus $T^{2}$. The line represents a fit of the low-temperature part with a Debyelike function $\Delta C / T=\gamma+\beta T^{2}$, which is used to estimate the AF magnon specific heat and velocity. Data taken from Cus03.

heat assuming a simple Debye model for its magnon contribution. Figure 4.6 shows a fit to the specific-heat data below $T=50 \mathrm{mK}$ with $\Delta C=C-C_{\mathrm{ph}}-C_{\mathrm{Q}}=\gamma T+\beta T^{3}$, with $C_{\mathrm{ph}}$ and $C_{\mathrm{Q}}$ being, respectively, the phonon and the nuclear quadrupole contributions. The huge electronic contribution $C_{\mathrm{el}}=\gamma T$ with $\gamma=1.64 \mathrm{~J} / \mathrm{K}^{2}$ mol denotes a heavy Fermi-liquid phase Cus03. The Debye-like term $C_{\mathrm{m}} \sim T^{3}$ describes long-wavelength AF acoustic magnons. From $\beta=132.2 \mathrm{~J} / \mathrm{K}^{4} \mathrm{~mol}$ one can extract the "magnetic" Debye temperature $\theta_{\mathrm{m}}$ and the group velocity $v_{\mathrm{m}}$ using the Debye model. In order to obtain a $\kappa_{\mathrm{m}}$ of $2-5 \cdot 10^{-3} \mathrm{~W} / \mathrm{Km}$ in the range $25-30 \mathrm{mK}$, as observed in Figure 4.4 , the corresponding magnon mean free path $l_{\mathrm{m}}$ has to be of the order of $10 \mu \mathrm{m}$. The low-temperature limit $l_{\mathrm{m}}$ is expected to be equal in size to that of the AF domains. Compared to other AF compounds (Cr: $16 \mu \mathrm{m}$ [Wer67], $\mathrm{UPt}_{3}$ : $1 \mu \mathrm{m}$ Vis98]), a few $\mu \mathrm{m}$ below about $0.03 \mathrm{~K}$, indeed, seems to be a reasonable order of magnitude. $\mathrm{YbRh}_{2} \mathrm{Si}_{2}$ is thus one of the rare cases where magnons can be observed not only as scattering centres but as heat carriers in a metal (e.g. Ref. Yel72; Mor84]). Usually their contribution is strongly suppressed by magnon-electron scattering.

Since magnons are bosons, their contribution to the specific heat and the thermal conductivity will vanish in the $T=0$ limit. But our lowest temperature of $25 \mathrm{mK}$ and even the $8 \mathrm{mK}$ reached in recent experiments Pou14 were not low enough to observe any decrease of this extra contribution. Instead, it grows substantially down to $8 \mathrm{mK}$, concealing the low-temperature behaviour of the electronic contribution $\kappa_{\mathrm{el}}$. However, the AF phase is a Fermi liquid. Hence, we expect the WF law to hold in the $T=0$ limit.

\section{Critical Field and Quantum Critical Regime}

While $\rho(T)$ is linear from $70 \mathrm{mK}$ up to $0.4 \mathrm{~K}$ for $B=0$, it bends for higher fields and the linear regime shrinks to below $0.12 \mathrm{~K}$ at the critical field. This non-linearity was also observed in other samples Rei14, Geg08; Wet08. Therefore, not only a linear fit but in addition a fit with a different power $\rho(T)=\rho_{0}^{\prime}+A^{\prime} T^{\alpha}$ was used to describe the data. The power was determined from $\rho(T)$ at the critical field $B_{\mathrm{c}}$ to $\alpha=0.78$. Both types of extrapolations are shown in Figure 4.4 . In particular, the $T^{0.78}$ fit can describe $\rho(T)$ well not only at $B_{\mathrm{c}}$ but in the whole field range $0 \leq B \leq 0.1 \mathrm{~T}$ above $T_{\mathrm{N}}$ and $T_{\mathrm{FL}}$. This regime is shown as the "quantum critical regime" in Figure 4.7. In the quantum critical regime, $\mathrm{YbRh}_{2} \mathrm{Si}_{2}$ shows the same properties as exactly at the critical field. Therefore, the $T=0$ 


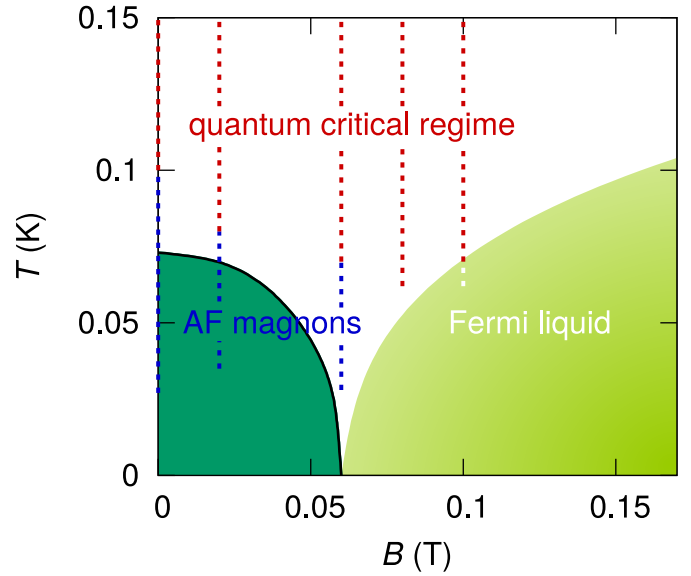

Figure 4.7: Regime for $T=0$ extrapolations around the QCP. The phase diagram of $\mathrm{YbRh}_{2} \mathrm{Si}_{2}$ is shown as a zoom of Figure 4.1 around the QCP. The dashed lines mark the temperature scans for the transport measurements shown in Figure 4.4. The data for $w$ and $\rho$ in Figure 4.4 indicate, that a magnon contribution in $w$ (blue) does not only exist in the $\mathrm{AF}$ phase, but also in the paramagnetic regime close to the phase boundary, especially at $B=B_{c}$. To obtain the $T=0$ limit of the electronic transport, the data above the onset of magnons are extrapolated (red). This extrapolation in the quantum critical regime can also be performed at fields slightly below and above $B_{c}$, since they capture the behaviour of the QCP as well. The quantum critical regime also extends to temperatures higher than $0.15 \mathrm{~K}$.

limit, which is extrapolated from this regime, captures the properties of the QCP.

Since $w(T)$ shows a similar dependence as $\rho(T)$, the same relations are used for its $T \rightarrow 0$ extrapolation in the quantum critical regime. For $B=0$ and $B=0.02 \mathrm{~T}$, this works well down to $0.1 \mathrm{~K}$ at which point the magnon contribution leads to a downturn of $w(T)$ as described above. Surprisingly, a similar downturn can be observed also at $B_{\mathrm{c}}$. The downturn cannot be captured by a $T^{0.78}$ fit and it extrapolates to $w(T)<\rho(T)$. Recent experiments confirm this crossing Pou14]. Hence, an extra contribution to the thermal conductivity similar to the AF phase appears also at $B_{\mathrm{c}}$. This can be interpreted as the contribution of overdamped magnons in the paramagnetic regime close to the QCP. Inelastic neutronscattering experiments showed that overdamped magnons exist for antiferromagnetically ordered materials substantially above the Néel temperature Wil83a; Wil83b; Mer14], and for antiferromagnetic quantum critical systems beyond the critical value of the tuning parameter [Arn11]. In addition, heat transport by short-lived magnon excitations has been reported, e.g., for $\mathrm{La}_{2} \mathrm{CuO}_{4}$, an 2D $S=1 / 2$ antiferromagnet with a Néel temperature $T_{\mathrm{N}} \approx 310 \mathrm{~K}$ Hes03. In particular, $S=1$ chain systems, such as $\mathrm{Y}_{2} \mathrm{BaNiO}_{5}$ (Ref. Kor06) and $\mathrm{Ni}\left(\mathrm{C}_{2} \mathrm{H}_{8} \mathrm{~N}_{2}\right)_{2} \mathrm{NO}_{2}\left(\mathrm{ClO}_{4}\right)$ (NENP) (Ref. [Sol08]) have served as model systems in this context.

As for $B=0$, the magnon contribution to $w(T)$ is expected to vanish in the $T \rightarrow 0$ limit also at $B_{\mathrm{c}}$. Unfortunately, it masks part of the temperature dependence of the electronic thermal conductivity at very low $T$, which is important for the investigation of the WF law. But it is reasonable to assume that $w(T)$ above the drop is entirely due to an electronic thermal conductivity and can be extrapolated towards $T=0$ in the same way as $\rho(T)$. As Figures 4.4 and 4.5 demonstrate, this procedure leads to $w_{0}-\rho_{0}>0$ and $L / \mathrm{L}_{0}<1$ in the zero-temperature limit not only at $B_{\mathrm{c}}$, but for extrapolations from the whole quantum critical regime which is defined above and sketched in Figure 4.7. Hence, we find a violation of the WF law at the $\mathrm{QCP}$ of $\mathrm{YbRh}_{2} \mathrm{Si}_{2}$.

A violation of the WF law has drastic physical consequences. We have seen in the previous sections that the WF law is valid in the Fermi-liquid regime and, hence, that quasiparticles 


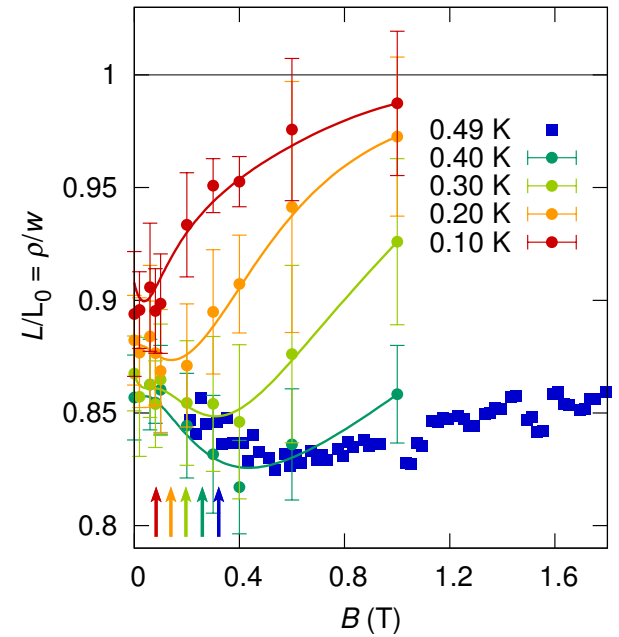

Figure 4.8: Magnetic field dependence of the Lorenz ratio. Dots and error bars are extracted from the temperature scans shown in Figure 4.4 Lines are guides to the eye. Squares represent an isothermal field scan for a second sample 63116_1\#1 used for the high-field study in Chapter 5 A clear minimum is visible, which shifts to higher $B$ for higher $T$ and, thereby, approximately follows the $T^{*}$ scale (arrows). No attempt was made to analyse lower temperature data due to the magnon contribution below $0.1 \mathrm{~K}$.

exist on either side of the QCP. A violation of the WF law at $B_{c}$ implies that these quasiparticles break up exactly at the QCP of $\mathrm{YbRh}_{2} \mathrm{Si}_{2}$. This puts strong constraints on every theory which aims to describe the QCP in $\mathrm{YbRh}_{2} \mathrm{Si}_{2}$. From the three theoretical concepts introduced in Section 4.2 only the Kondo-breakdown scenario implies a description beyond quasiparticles.

\section{Isothermal Field Dependence of the Lorenz Ratio}

The magnetic-field dependence of the Lorenz ratio between $0.1 \mathrm{~K}$ and $0.4 \mathrm{~K}$ was extracted from the data in Figure 4.5 and is plotted in Figure 4.8. $L(B) / \mathrm{L}_{0}$ shows a shallow minimum. Its position shifts to higher fields with higher temperature and lies within the FWHM of the $T^{*}$ line. The minimum narrows as the temperature is reduced, but no attempt was made to quantify this dependence due to the large error. An isothermal field-dependent measurement of the Lorenz ratio on another sample of better quality (RRR of 150) is included. Measured at slightly higher temperature, it nicely confirms the evolution of the minimum in $L(B) / \mathrm{L}_{0}$.

\subsubsection{Comparison with Results from Other Groups}

Since there is controversy as to whether the WF law is violated or not at the QCP in $\mathrm{YbRh}_{2} \mathrm{Si}_{2}$, we will compare the different experimental results of all groups investigating this topic. Figure 4.9 summarizes the results for $w(T), \rho(T)$ and $L(T) / \mathrm{L}_{0}$ at $B=0$ and $B_{\mathrm{c}}$ of our investigation and from the publications Machida et al. Mac13, Reid et al. Rei14], Pourret et al. Pou14, and Tomokuni et al. Tom11. The different field configurations, sample qualities, and the relations used to scale $w(T)$ and $\rho(T)$ are listed in Table 4.2 .

Figure 4.9(a) demonstrates that $w(T)$ and $\rho(T)$ from all measurements can be scaled almost perfectly on top of each other using a constant factor plus a correction for the residual resistivity. The same scaling is used for both $w(T)$ and $\rho(T)$. There are only minor differences in the AF phase, which do not change the general picture.

The curves for $B_{\mathrm{c}}$ in Figure 4.9(b) are scaled with the same relations as for $B=0$. 

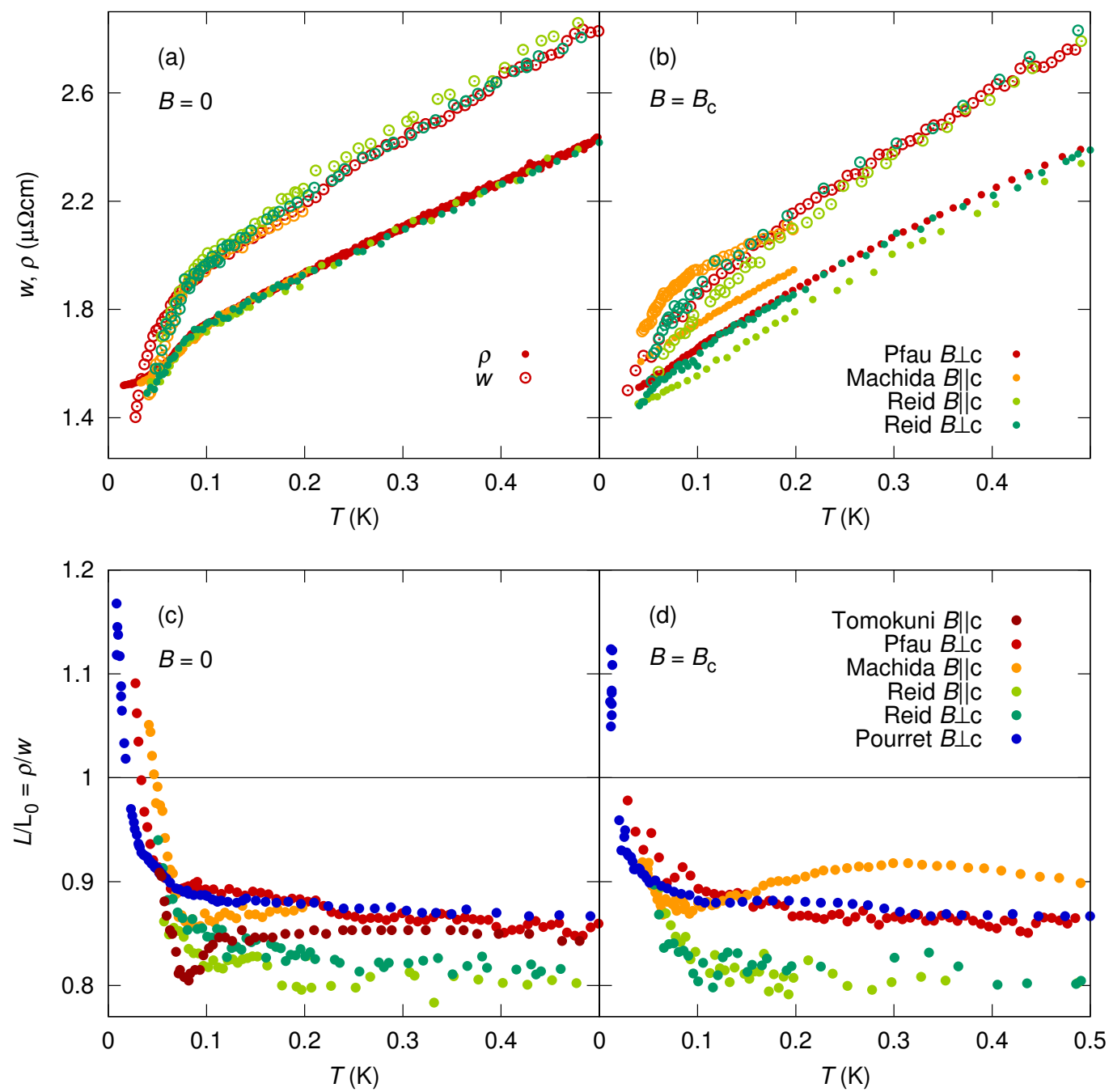

Figure 4.9: Comparison of our results with data published by other groups. The top panels show the thermal and electrical resistivities at $B=0$ (a) and approximately at the critical field (b), which is $0.07 \mathrm{~T}$ for $B \perp c$ and $0.66 \mathrm{~T}$ for $B \| c$. At $B=0$, the data from the other groups can nicely be scaled on top of ours using the relations in Table 4.2 The same scaling is used in (b). (c) and (d) show the Lorenz ratio $L / \mathrm{L}_{0}$ for the same but unscaled data sets adding Pourret et al. and Tomonuki et al.. All of them show the same tendency in $L / \mathrm{L}_{0}$ with a flat part above $0.1 \mathrm{~K}$ and a steep increase below $0.1 \mathrm{~K}$ when the magnon contribution sets in. All data are extracted from the following references with the following fields used for the plots (b),(d): Pfau et al. $B=0.06 \mathrm{~T}$, Machida et al. $B=0.66 \mathrm{~T}$ Mac13, Reid et al. $B=0.6 \mathrm{~T}$ and $0.06 \mathrm{~T}$ Rei14], Pourret et al. $B=0.07 \mathrm{~T}$ Pou14], Tomokuni et al. Tom11]. $L / \mathrm{L}_{0}$ from Reid et al. was calculated using the extracted values for $w$ and $\rho$. From Tomokuni et al., only $L / \mathrm{L}_{0}$ at $B=0$ was included due to the large noise level in the other data. 


\begin{tabular}{lllllll}
\hline & group & sample & field & $\begin{array}{l}\text { residual } \\
\text { resistivity }\end{array}$ & RRR & $\begin{array}{l}\text { scaling of } \rho, w \text { to } \\
\text { match Pfau } \text { et al. }\end{array}$ \\
\hline & Pfau et al. & 1 & $B \perp c$ & $1.5 \mu \Omega \mathrm{cm}$ & 50 & 1 \\
& & 2 & $B \perp c$ & $1.1 \mu \Omega \mathrm{cm}$ & 70 & $1.25 \rho+0.22 \mu \Omega \mathrm{cm}$ \\
Mac13 & Machida et al. & & $B \| c$ & $0.9 \mu \Omega \mathrm{cm}$ & 90 & $1.3(\rho, w)+0.31 \mu \Omega \mathrm{cm}$ \\
\hline Rei14 & Reid et al. & $\mathrm{A}$ & $B \perp c$ & $0.75 \mu \Omega \mathrm{cm}$ & 105 & $1.5(\rho, w)+0.5 \mu \Omega \mathrm{cm}$ \\
& & $\mathrm{B}$ & $B \| c$ & $0.66 \mu \Omega \mathrm{cm}$ & 120 & $1.25(\rho, w)+0.5 \mu \Omega \mathrm{cm}$ \\
Pou14 & Pourret et al. & & $B \perp c$ & $1.7 \mu \Omega \mathrm{cm}$ & 65 & \\
\hline Tom11 & Tomokuni et al. & & $B \| c$ & $0.85 \mu \Omega \mathrm{cm}$ & 90 & \\
\hline
\end{tabular}

Table 4.2: List of all published measurements, which were performed to test the WF law. They differ in field orientation and sample quality. The latter is expressed in terms of residual resistivity and RRR. For the sample used in Pou14, these values are taken from Tau13. Figure 4.9 compares the data of these measurements.

Although they deviate a little more, the overall behaviour is the same for all measurements: $\rho(T)$ follows a power law slightly less than linear down to lowest $T$, while $w(T)$ deviates from this behaviour at around $70 \mathrm{mK}$, drops and starts to cross $\rho(T)$.

Figures 4.9 (c) and (d) compare the Lorenz ratio. The results for $B=0$ and $B_{\mathrm{c}}$ look almost identical. Deviations between the groups are minor and are restricted to slight variations of the absolute value and slight differences in the curvature. The overall picture, however, is the same for all measurements: an almost constant Lorenz ratio of $L / \mathrm{L}_{0}=0.85 \pm 0.05$ between $0.1 \mathrm{~K}$ and $0.5 \mathrm{~K}$ and a sharp increase towards $L / \mathrm{L}_{0}>1$ below $0.1 \mathrm{~K}$. The crossing of $L / \mathrm{L}_{0}=1$ is observed for measurements which reached low-enough temperatures.

To answer the question whether the WF law is valid or violated, an extrapolation of $w(T)$ and $\rho(T)$ towards $T=0$ is necessary. The nice agreement between all measurements shows that the groups only differ in the way they interpret and extrapolate their data.

We ascribe the upturn of $L / \mathrm{L}_{0}$ below $0.1 \mathrm{~K}$ to a magnon contribution for both fields $B=0$ and $B=B_{\mathrm{c}}$. However, we observe a clear $L / \mathrm{L}_{0}>1$ only for $B=0$. Due to the extra magnon contribution, we extrapolate only the data at temperatures above the onset of this contribution. This analysis indicates a violation of the WF law. In the studies by Reid et al. and Machida et al., which followed our publication, the upturn in $L / \mathrm{L}_{0}$ was also observed, but similar to our study $L / \mathrm{L}_{0}$ stayed smaller than 1 in the measured temperature range at the critical field $B_{\mathrm{c}}$. In contrast to our interpretation, these authors associate the upturn in $L / \mathrm{L}_{0}$ at $B_{\mathrm{c}}$ not with a sign of additional heat carriers and, therefore, use the data below $0.1 \mathrm{~K}$ as a basis for an extrapolation to $T=0$. These extrapolations result in $L / \mathrm{L}_{0}=1$ at $T=0$. However, a crossing of $w(T)$ and $\rho(T)$ was indeed observed also at the critical field $B_{\mathrm{c}}$ in the most recent investigation by Pourret et al., which includes data at temperatures as low as $8 \mathrm{mK}$. This confirms our interpretation of an additional heat channel. In the light of these new findings, the extrapolation procedure in the reports of Reid et al. and Machida et al. appears questionable. Although Pourret et al. also express their doubts concerning a violation of the WF law, no reliable alternative extrapolation procedure is available to date. This is due to the difficulties to analyse the additional contribution to the heat transport. Only a deeper understanding of this extra contribution can settle the debate about whether the WF law is valid or violated in $\mathrm{YbRh}_{2} \mathrm{Si}_{2}$. 


\subsection{Conclusion}

We performed measurements of the electrical and the thermal conductivity to test the WF law across the field-tuned $\mathrm{QCP}$ in $\mathrm{YbRh}_{2} \mathrm{Si}_{2}$. Using an elaborate set-up, we were able to reach temperatures as low as $25 \mathrm{mK}$. These turned out essential to develop a conclusive concept for the zero-temperature extrapolation. Several other groups performed similar experiments, a very recent one reaching even $8 \mathrm{mK}$ [Pou14]. All of them confirm our data in the respective parameter range.

We could clearly confirm the Wiedemann Franz law in the Fermi-liquid phase at magnetic fields above the QCP. In the AF phase, we detected an extra contribution to the thermal transport, which we identified as due to (para-)magnon heat transport. It sets in at approximately $100 \mathrm{mK}$ slightly above the Néel temperature and grows down to $8 \mathrm{mK}$. Up to now, a decrease of this magnon thermal conductivity could not be observed. However, we expect it to vanish at $T=0$ due to the bosonic character of the magnons. Unfortunately, it masks the thermal transport of the electronic quasiparticles in the AF phase, which prohibits an extrapolation to $T=0$. But we believe that the WF law holds here as well due to the Fermi-liquid character of the AF phase.

A similar extra contribution also appears at the critical field. We assign it to overdamped paramagnons, which implies that it vanishes at $T=0$. Again, this contribution masks the low-temperature behaviour of the electronic quasiparticles. We argue that an extrapolation of the quantum critical regime right above the onset of this extra contribution captures the behaviour at the QCP. This extrapolation indicates a violation of the WF law with a Lorenz ratio $L / \mathrm{L}_{0}$ of approximately 0.9 at the QCP.

The way how the experimental data should be extrapolated to zero temperature - and consequently the validity of our result - is highly debated among the different groups. Meanwhile, the presence of an extra contribution to the thermal transport in the AF phase and at the critical field is clearly confirmed. It vanishes in the paramagnetic regime for fields $B \geq 0.2 \mathrm{~T}$ within our accessible temperature window. However, only a deeper understanding of the extra contribution can ultimately resolve this issue.

A violation of the WF law as suggested by our analysis has dramatic physical consequences. This law is a fundamental result of the presence of quasiparticles. Hence, its validity permits the description of a system - whether it is a Fermi liquid or a non-Fermi liquid - in terms of quasiparticles. Conversely, a violation of the WF law indicates a breakdown of this quasiparticle concept and puts very strong constraints on a theoretical description. From the three concepts for the quantum criticality in $\mathrm{YbRh}_{2} \mathrm{Si}_{2}$, which are mentioned above, only the Kondo-breakdown scenario implies a description beyond Landau quasiparticles. 


\section{Kondo Lattices in Magnetic Field}

This chapter is devoted to Kondo-lattice systems in magnetic fields for which the Zeeman energy reaches the order of the Kondo temperature. We studied $\mathrm{CeRu}_{2} \mathrm{Si}_{2}$ and $\mathrm{YbRh}_{2} \mathrm{Si}_{2}$. In both compounds, transitions were reported at $8 \mathrm{~T}$ and $10 \mathrm{~T}$, respectively, the origins of which are debated. We investigate the development of the quasiparticle bands of the these two Kondo lattices as a function of magnetic fields in general and study the reported transitions in particular. For this purpose, detailed field-dependent thermopower, resistivity, and thermal-conductivity measurements were performed. For $\mathrm{CeRu}_{2} \mathrm{Si}_{2}$, we demonstrate that the thermopower is consistent with a Lifshitz transition predicted by previous electrical-transport studies. However, rigid-band models seem insufficient to account for thermodynamic observations and a satisfying description is still missing. For $\mathrm{YbRh}_{2} \mathrm{Si}_{2}$, our measurements reveal in total three transitions at high fields instead of a single one at $10 \mathrm{~T}$. We find an unexpected validity of the Mott formula above $2 \mathrm{~T}$ and an extraordinarily good agreement of field-dependent renormalized band (RB) structure calculations with experimental results. They allow to identify the observed transitions as Lifshitz transitions, which are superposed on a smooth suppression of the Kondo effect in magnetic field. Field-dependent transport measurements turned out as an excellent method for studying the validity of Mott's formula in heavy-fermion systems and for identifying Lifshitz transitions. Most of the following work was published in Refs. Pfa12a Pfa13.

\subsection{Introduction and Motivation}

Over the past years, so-called metamagnetic transitions were reported in many heavyfermion systems $[$ Löh93, Sug90, Sec86, Fra84, Oda94]. The term "metamagnetism" is commonly used for magnetic-field-induced phase transitions, which lead to a strong increase of the magnetization. However, in Kondo systems - as in the following - "metamagnetism" and "metamagnetic transition (MMT)" mostly refer to a crossover-like, superlinear rise in $M(B)$ around a magnetic field of the order of $10 \mathrm{~T}$. The origin of these transitions, however, can be very diverse and is unclear in many cases. Sometimes it is related to a line of first-order transitions, which terminates at a critical end point at a certain temperature, above which metamagnetic behaviour occurs Uhl04: Tau10.

$\mathrm{CeRu}_{2} \mathrm{Si}_{2}$ is the prototype example of metamagnetism in Kondo-lattice systems. Its transition around $8 \mathrm{~T}$ was discovered 30 years ago, but its origin is still under debate. The Kondo-lattice compound $\mathrm{YbRh}_{2} \mathrm{Si}_{2}$ also exhibits a field-induced transition at $10 \mathrm{~T}$, however its signatures are quite different with a kink in the magnetization instead of a strong increase. Despite their different phenomenology, very similar models have been proposed for both cases. The first one is an abrupt destruction of the Kondo effect and a breakup of the heavy composite quasiparticles. In this model, the Fermi surface would include the $f$ degrees 
of freedom only on the low-field side of the transition. The second model is a topological change of the Fermi surface called Lifshitz transition (for an introduction see Section 2.5). The study of Lifshitz transitions is of particular importance as they might be connected to the onset of superconductivity Liu10, Yel11, LeB11. Moreover, they are discussed in metamagnetic ferromagnets Kot11; Yam07] and observed within the hidden-order phase of $\mathrm{URu}_{2} \mathrm{Si}_{2}$ Mal11, Alt11].

While these two interpretations - the destruction of the Kondo effect and Lifshitz transitions - seem quite different at first sight, they essentially rely on the Kondo-lattice model: Only in the presence of the very flat bands of the composite fermions, the Zeeman splitting can induce such large effects on the Fermi surface at moderate fields as observed in $\mathrm{CeRu}_{2} \mathrm{Si}_{2}$ and $\mathrm{YbRh}_{2} \mathrm{Si}_{2}$. A splitting simultaneously leads to a continuous decrease of the quasiparticle mass. Since the bandwidth of the flat bands as well as the strength of the Kondo effect is connected to the energy scale $T_{\mathrm{K}}$, also the field scale for both processes is related to $T_{\mathrm{K}}$ Tok05, Ber12. Therefore, a detailed knowledge of the field evolution of the composite quasiparticles in the Kondo lattice and their quasiparticle bands is a prerequisite to get a deeper understanding of such transitions.

The evolution of the quasiparticle bands in magnetic field is important in more general terms, e.g. in the interpretation of de Haas-van Alphen (dHvA) experiments, which have contributed a large amount to the current knowledge on heavy-fermion systems. They need to be performed at very high fields, however band structure calculations necessary to interpret these data almost exclusively exist at zero field.

To study the transitions in $\mathrm{CeRu}_{2} \mathrm{Si}_{2}$ and $\mathrm{YbRh}_{2} \mathrm{Si}_{2}$ in particular and the field evolution of the composite quasiparticles in general, we performed field-dependent thermopower, resistivity and thermal-conductivity measurements. Transport coefficients are very sensitive to quasiparticle properties and to changes in the Fermi surface. We put particular focus on magnetic-field-dependent isothermal thermopower and resistivity and analyse these data in detail using different approaches: We compare them to renormalized band (RB) structure calculations and rigid-band models and we test the validity of the Mott formula.

The chapter is organized as follows: We will first describe the experimental method (Section 5.2, before we introduce the specifics of the two compounds and show the related experimental results from our transport measurements. We will start with $\mathrm{CeRu}_{2} \mathrm{Si}_{2}(\mathrm{Sec}-$ tion 5.3 and continue with $\mathrm{YbRh}_{2} \mathrm{Si}_{2}$ (Section 5.4). For $\mathrm{YbRh}_{2} \mathrm{Si}_{2}$, we will also compare the experimental results with elaborate RB structure calculations. Since these calculations are quite demanding and not available e.g. for $\mathrm{CeRu}_{2} \mathrm{Si}_{2}$ at the moment, we will also apply more simple rigid-band models in Section 5.5. We want to illuminate their applicability in particular for the analysis of our data on both compounds and in general for the detection of Lifshitz transitions. Further on, we will use the advantage of field-dependent measurements to study the validity of Mott's formula in these two systems and across their high-field transitions in Section 5.6 .

\subsection{Experimental Methods}

Thermal- and electrical-transport properties were measured on the "SHE" set-up using the standard one-heater-two-thermometers technique as described in Chapter 3. Low-resistance contacts to the samples were made by soldering. In order to avoid the high thermal resistance of superconducting contacts used in the experimental stage, the minimum magnetic field we 
applied was $0.2 \mathrm{~T}$. We applied a maximum field of $12 \mathrm{~T}$ and studied the temperature range between $100 \mathrm{mK}$ and $700 \mathrm{mK}$. The current and magnetic field were reversed to check for thermoelectric contributions to the DC resistivity, but these were found to be negligible for the currents employed $(10 \mu \mathrm{A}<I<150 \mu \mathrm{A})$. This was also a useful check to confirm that transverse transport properties (i.e. Hall, thermal Hall and Nernst effects) made no detectable contribution to the measurements. With this set-up, we studied two compounds: $\mathrm{CeRu}_{2} \mathrm{Si}_{2}$ and $\mathrm{YbRh}_{2} \mathrm{Si}_{2}$.

The sample of $\mathrm{CeRu}_{2} \mathrm{Si}_{2}$ was grown by F.S. Tautz in Cambridge. The residual resistivity, $\rho_{0}$, was $1.1 \mu \Omega \mathrm{cm}$, compared to $0.4 \mu \Omega \mathrm{cm}$ for a sample from the same batch measured in Ref. Dao06a. Resistivity, thermopower, and thermal conductivity were measured on a single crystal of dimensions $3.5 \times 0.28 \times 0.08 \mathrm{~mm}^{3}(a \times b \times c)$. The magnetic field was applied along the crystallographic $c$ axis, while thermal and electrical currents were applied along the $a$ axis.

In the case of $\mathrm{YbRh}_{2} \mathrm{Si}_{2}$, two high-quality samples from the same batch were investigated, with residual resistivities of approximately $0.5 \mu \Omega \mathrm{cm}$. On sample \#1 (63116_1) simultaneous DC-resistivity, thermal-conductivity, and thermopower measurements were performed. Sample \#2 (63116_2) was used to extend the resistivity data down to $0.03 \mathrm{~K}$ on another setup applying an AC technique by T. Westerkamp. The magnetic field $B$ was always applied perpendicular to the $c$ axis. The currents for resistivity and thermal transport were parallel to $B$.

\section{3 $\mathrm{CeRu}_{2} \mathrm{Si}_{2}$}

$\mathrm{CeRu}_{2} \mathrm{Si}_{2}$, as well as $\mathrm{YbRh}_{2} \mathrm{Si}_{2}$, crystallizes in the tetragonal $\mathrm{ThCr}_{2} \mathrm{Si}_{2}$ structure (see Figure 4.1 in Chapter 4p. It is a Kondo-lattice system, which has an uncomplicated phase diagram without any order such as magnetism or superconductivity. For a good review of the many thermodynamic, transport, and spectroscopic experiments on $\mathrm{CeRu}_{2} \mathrm{Si}_{2}$, see Ref. Flo02.

\subsubsection{The Metamagnetic Transition}

$\mathrm{CeRu}_{2} \mathrm{Si}_{2}$ attracted much interest due to a large anomaly in all thermodynamic properties when a magnetic field of $B_{\mathrm{MMT}} \approx 8 \mathrm{~T}$ is applied along the crystallographic $c$ axis. The most obvious effects are a rapid non-linear rise in the magnetization and a pronounced peak in the electronic specific heat coefficient $\gamma$. The thermodynamics of this MMT is consistent with the proximity to a quantum critical end point Wei10; Mil02. In the low-temperature limit, however, the material exhibits Fermi-liquid-like properties at all magnetic fields and the MMT remains always a crossover. Despite of more than 30 years of research on this compound, the origin of the MMT is still under debate.

First dHvA measurements across the transition detected an abrupt change in the oscillation frequency at $B_{\mathrm{MMT}}$. Together with a decrease in the $\gamma$ coefficient, this was interpreted in terms of a destruction of the composite fermions above $B_{\mathrm{MMT}}$ Aok93. Later on, the maximum in the specific heat coefficient could be modelled using a simple rigid-band approach, where a peak in the density of states (DOS) moves through the Fermi energy Aok98. Meanwhile, low-temperature Hall-effect measurements showed a very small but distinct negative dip that appears to be sharper than the thermodynamic signatures of the MMT Dao06a]. This was interpreted as a sign of a topological transition of the Fermi surface, driven by 
enhanced Zeeman splitting of the heavy-fermion bands. Indeed, spin-splitting and a considerable spin dependence of the quasiparticle masses was directly detectable via dHvA measurements in some of the lighter bands Dao06b, Tak96.

To shed more light on the origin of the MMT and to study the magnetic-field development of the composite quasiparticles in $\mathrm{CeRu}_{2} \mathrm{Si}_{2}$, we performed detailed magnetic-field-dependent electrical- and thermal-transport measurements. We repeated the magnetoresistance measurements from Dao06a to pin down the transition field $B_{\mathrm{MMT}}$ and investigated in detail the thermopower $S(B)$ around $B_{\mathrm{MMT}}$.

\subsubsection{Transport Data}

Figure 5.1 gives an overview of our measurement results, which we will describe in detail in the following. It also includes a comparison to results obtained by other groups.

Figure 5.1(b) presents the magnetoresistivity $\rho(B)$, which increases with increasing magnetic field $B$. The small maximum at higher temperatures around the MMT disappears for lower $T$, where we observe a monotonic behaviour with a step-like increase in $\rho(B)$ around the MMT. These observations are in accordance with those reported in Dao06a. The lowest-temperature data are close to the residual resistivity $\rho_{0}$. Apart from the ordinary magnetoresistance arising from cyclotron motion of the electrons, a variation of $\rho_{0}$ with magnetic field can arise either from changes in the shape of the Fermi surface (orbital contributions) or from modification of the impurity potential, perhaps due to enhanced zero-temperature fluctuations Miy02. Calculations based on this second mechanism in the vicinity of a ferromagnetic quantum critical point show that the fluctuations generate a peak in $\rho_{0}$ at the critical field. We know that the ferromagnetic fluctuations in $\mathrm{CeRu}_{2} \mathrm{Si}_{2}$ are also peaked around the MMT Sat04]. $\rho_{0}$ shows a monotonic increase, however, suggesting that the orbital mechanism is more important.

We will use a comparison of $\rho(B)$ to previous data from Ref. Dao06a to pin down the transition field $B_{\mathrm{MMT}}$. Examining the data from Ref. Dao06a more carefully, we see that $d \rho / d B$ peaks at precisely the same field as that where the small negative dip in the Hall resistivity occurs. In the current data, the peak in $d \rho / d B$ (not shown, see Ref. Pfa12a]) is at $B_{\mathrm{MMT}}=7.80 \pm 0.05 \mathrm{~T}$.

The temperature dependence of the resistivity $\rho(T)$ is Fermi-liquid-like at low temperatures as in previous studies, i.e., $\rho=\rho_{0}+A T^{2}$ at all fields. Figure 5.1(a) shows $A$ extracted from temperature sweeps. $A$ is expected to be proportional to the square of the electronic specific-heat coefficient $\gamma$ according to the Kadowaki-Woods relationship Kad86 and is, hence, a thermodynamic property. This relationship has been seen to hold well in other samples Kam96, Aok11]. $B_{\text {MMT }}$ determined from $\rho(B)$ coincides with the peak in $A$ within the resolution of the measurement. Hence, the transition appears at the same field in transport and in thermodynamics.

Figure 5.1(d) shows the Lorenz ratio $L(B) / \mathrm{L}_{0}$. It relates the electrical and thermal conductivity via the Wiedemann-Franz (WF) law $L / \mathrm{L}_{0}=\kappa / \mathrm{L}_{0} \sigma T$ where $L_{0}=\pi^{2} k_{B}^{2} / 3 e^{2}$ is Sommerfeld's constant. This ratio holds to within $5 \%$ for all fields at $100 \mathrm{mK}$, as we might expect in the Fermi-liquid regime. Previous studies down to $0.64 \mathrm{~K}$ were not able to test the validity of the $\mathrm{WF}$ in the low-temperature limit Ama89, Ser97.

Figure 5.1(c) shows the isothermal thermopower of $\mathrm{CeRu}_{2} \mathrm{Si}_{2}$ as $S(B) / T$, which has a complex field dependence with multiple sign changes reflecting the multi-band electronic structure Ama89]. The sign of the thermopower is often used as an indicator of the sign 


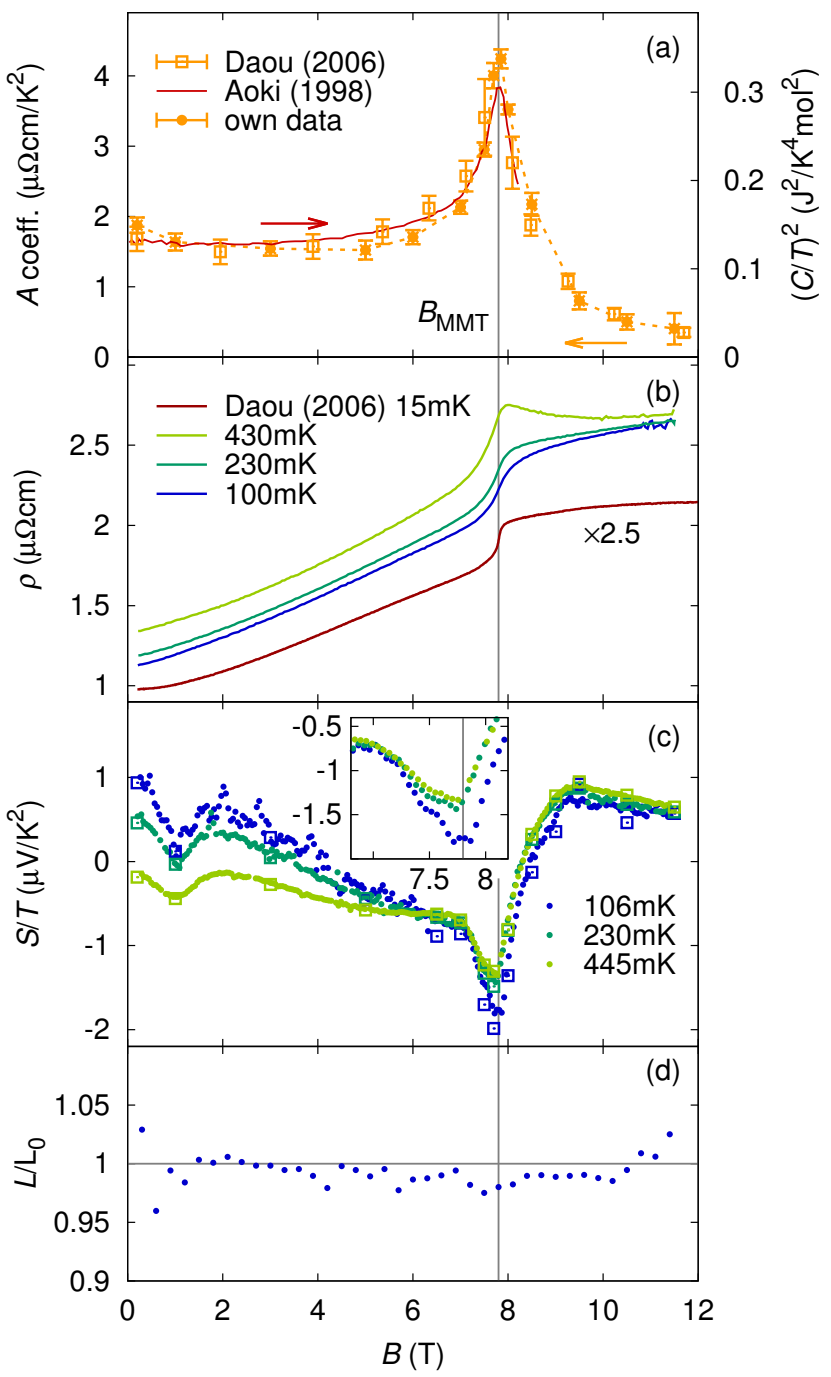

Figure 5.1: Properties of $\mathrm{CeRu}_{2} \mathrm{Si}_{2}$ as a function of magnetic field $B$. (a) The $A$ coefficient of the resistivity, $\rho$, peaks at $B_{\mathrm{MMT}}=(7.80 \pm 0.05) \mathrm{T}$. For comparison, similar data is included for the sample of Ref. Dao06a and also published specific-heat data on another sample Aok98. (b) A peak in $\rho(B)$ appears at higher temperatures. The resistivity measured at $100 \mathrm{mK}$ is close to the residual resistivity $\rho_{0}$, however, and shows no such peak at the MMT. We compare our results with those from Ref. Dao06a, the data of which have been slightly scaled: $\rho$ values by a factor of 2.5 and $B$ values so that $B_{\mathrm{MMT}}$ matches, accounting for a misalignment of the sample. The MMT appears much broader in $\rho$ of the present sample. (c) The thermopower divided by temperature for field sweeps (filled circles) and temperature sweeps (open squares) is in excellent agreement. $S / T$ shows multiple sign changes and a strong negative peak near the MMT. There is a sharp kink in the thermopower precisely at $B_{\mathrm{MMT}}$ which is more clearly seen in the inset. An additional peak at $1 \mathrm{~T}$ becomes sharper as the temperature is reduced. (d) The Lorenz ratio at $100 \mathrm{mK} . L / \mathrm{L}_{0}=1$ within the noise level of our measurement. 
of the dominant carriers. An electron band would make a negative contribution to the thermopower, and a hole band a positive contribution. In a multiband system, however, the sum of the contributions to the thermopower is weighted by the band conductivity, and $S$ can acquire either sign depending on the details of the band structure. In addition, sign changes in the thermopower are often used to detect points of transitions in a system. Buhmann et al. recently showed, however, that also in a very simple one-band tight-binding model, the thermopower shows sign changes on non-specific points as a function of band filling Buh13. Hence, we expect sign changes in the thermopower in $\mathrm{CeRu}_{2} \mathrm{Si}_{2}$, but they do not necessarily determine points of transitions.

Close to $B_{\mathrm{MMT}}=7.8 \mathrm{~T}$, which we determined by our magnetoresistance and specific-heat measurements, the thermopower shows a broad negative peak, apparently at a field slightly below $B_{\mathrm{MMT}}$. Precisely at $B_{\mathrm{MMT}}$, there is a sharp kink in the thermopower. $S(B) / T$ changes slope suddenly, within the resolution of our measurement of about $0.05 \mathrm{~T}$. This feature is more clearly seen in the inset of Figure 5.1(c). The kink is resolved up to the highest temperature that we measured $(0.57 \mathrm{~K})$. This temperature dependence is similar to that of the narrow feature in the Hall effect at $B_{\mathrm{MMT}}$, which is clear and essentially unchanged below $0.5 \mathrm{~K}$ Dao06a].

There is an additional peak at $1 \mathrm{~T}$, that becomes sharper at low temperatures. It is not associated to any known feature in other transport or thermodynamic properties. This peak is not resolved in measurements at $1.5 \mathrm{~K}$ Bel04, but could recently be confirmed by thermopower measurements in Ref. Bou14.

We will discuss the features at $B_{\mathrm{MMT}}$ in all quantities later on in detail, but keep the discussion short for the other transition, since thermopower is so far the only quantity which detected it.

\section{$5.4 \mathrm{YbRh}_{2} \mathrm{Si}_{2}$}

An introduction to some general properties of $\mathrm{YbRh}_{2} \mathrm{Si}_{2}$ is given in Section 4.1 in the previous chapter, where we discuss the validity and violation of the Wiedemann-Franz law at the magnetic-field-induced quantum critical point (QCP).

\subsubsection{The High-Field Transition}

$\mathrm{YbRh}_{2} \mathrm{Si}_{2}$ also exhibits a high-field transition, although not a prototypical MMT with an S-shape in $M$, but with a kink structure at $B_{0}=10 \mathrm{~T}$ Geg06]. Specific-heat, susceptibility and magnetostriction measurements indicate a rapid decrease of the effective mass across $B_{0}$ Tok04 Tok05 Geg06]. Together with a scaling of $B_{0}$ with the Kondo temperature $T_{\mathrm{K}}$ under pressure, this was initially interpreted as a breakdown of the Kondo screening with decomposed quasiparticles on the high-field side. Later, dHvA experiments were interpreted in terms of a Lifshitz transition at $B_{0}$, where a spin-split band disappears Rou08, Lif60; Ber12. The transition field of a Lifshitz transition in a Kondo lattice would also scale with $T_{\mathrm{K}}[$ Ber12].

To elucidate the origin of the transition at $10 \mathrm{~T}$ in $\mathrm{YbRh}_{2} \mathrm{Si}_{2}$, we performed detailed measurements of the field-dependent resistivity, thermopower and thermal conductivity. Additional Hall-effect measurements, which were performed at our institute on a sample from the same batch, are published in Ref. [Nar13] but will not be discussed in this thesis. 
The experimental results are compared with predictions from RB structure calculations by G. Zwicknagl. The analysis and discussion focusses on the field and temperature region within the paramagnetic Fermi-liquid regime above $2 \mathrm{~T}$ away from the QCP, which was discussed in Chapter 4.

\subsubsection{Transport Data}

Figure 5.2(a) shows the magnetic-field-dependent specific heat as $C(B) / T$ and the susceptibility $\chi(B)$ of $\mathrm{YbRh}_{2} \mathrm{Si}_{2}$ taken from Ref. Tok05. $\chi(B)$ was measured at $40 \mathrm{mK}, C(B) / T$ was determined from zero-temperature extrapolations of the electronic specific heat $C(T)$ at different fields. They fall on top of each other and their field dependence mimics that of the effective mass. Clearly visible is the monotonic decrease of both with increasing field and their drop across $B_{0}=10 \mathrm{~T}$.

Figures 5.2(b) and (c) present our measurements of the low-temperature magnetoresistivity $\rho(B)$ and the isothermal, field-dependent thermopower $S(B) / T$, respectively. The Lorenz ratio $L(B) / \mathrm{L}_{0}$ obtained from magnetoresistance and thermal conductivity $\kappa$ is shown in Figure 5.2 (d) only for $490 \mathrm{mK}$, because of an enhanced noise level at lower temperatures. Considering the $B-T$ phase diagram of $\mathrm{YbRh}_{2} \mathrm{Si}_{2}$ Geg06], it is natural to relate the low-field $(B<2 \mathrm{~T})$ behaviour of all these quantities to the signatures of the QCP and the surrounding non-Fermi-liquid regime. These signatures are in agreement with previously reported results: the step in magnetoresistance Geg07], the pronounced minimum in $S(B) / T$ Har10b] and the minimum of the isothermal Lorenz ratio Pfa12b (see also Chapter 4) are clearly visible.

Next, we focus on the high-field properties beyond $2 \mathrm{~T}$, where $\mathrm{YbRh}_{2} \mathrm{Si}_{2}$ is a Fermi liquid below $200 \mathrm{mK}$. The key features are three transitions. As in $\mathrm{CeRu}_{2} \mathrm{Si}_{2}$, we determine their exact position again with the help of the magnetoresistivity $\rho(B)$, which shows three tiny kinks at $B_{1}=(3.4 \pm 0.1) \mathrm{T}, B_{2}=(9.3 \pm 0.1) \mathrm{T}$, and $B_{3}=(11.0 \pm 0.2) \mathrm{T}$. The transitions are more obvious in the thermopower: $S(B) / T$ shows three pronounced steps at these fields, which become sharper at lower $T$. The position of $B_{1}, B_{2}$, and $B_{3}$ is sample and temperature independent: The resistivity of three measured samples including the one for Hall-effect studies exhibits kinks at the same position. Recently, Pourret et al. Pou13 also performed thermopower and resistivity measurements on a sample from a different batch. Their results confirm our measurements. Additionally, they claim to observe more than three transitions. Indeed, there is some additional fine structure also visible in our data of $\rho(B)$, however, we will concentrate in the following on the far strongest features at $B_{1}, B_{2}$, and $B_{3}$.

In summary, our transport studies reveal that, in contrast to previous reports on thermodynamic properties Tok04, Tok05, Geg06], the transition at $B_{0}=10 \mathrm{~T}$ is actually composed of two well-separated features at $9.3 \mathrm{~T}$ and $11.0 \mathrm{~T}$, which do not merge in the extrapolation $T \rightarrow 0$. Additionally, we resolve another transition at $3.4 \mathrm{~T}$, which was also not observed previously.

\subsubsection{Renormalized Band Structure Calculations for $\mathrm{YbRh}_{2} \mathrm{Si}_{2}$}

Our experimental data on $\mathrm{YbRh}_{2} \mathrm{Si}_{2}$ together with previous results on this compound already indicate the complex nature of the band-structure effects responsible for $B_{1}, B_{2}$, and $B_{3}$. For example, the transition around $B_{0}$ is actually composed of two transition fields and the Sommerfeld coefficient $\gamma(B)$ decreases only moderately between them from $250 \mathrm{~mJ} / \mathrm{molK}^{2}$ 


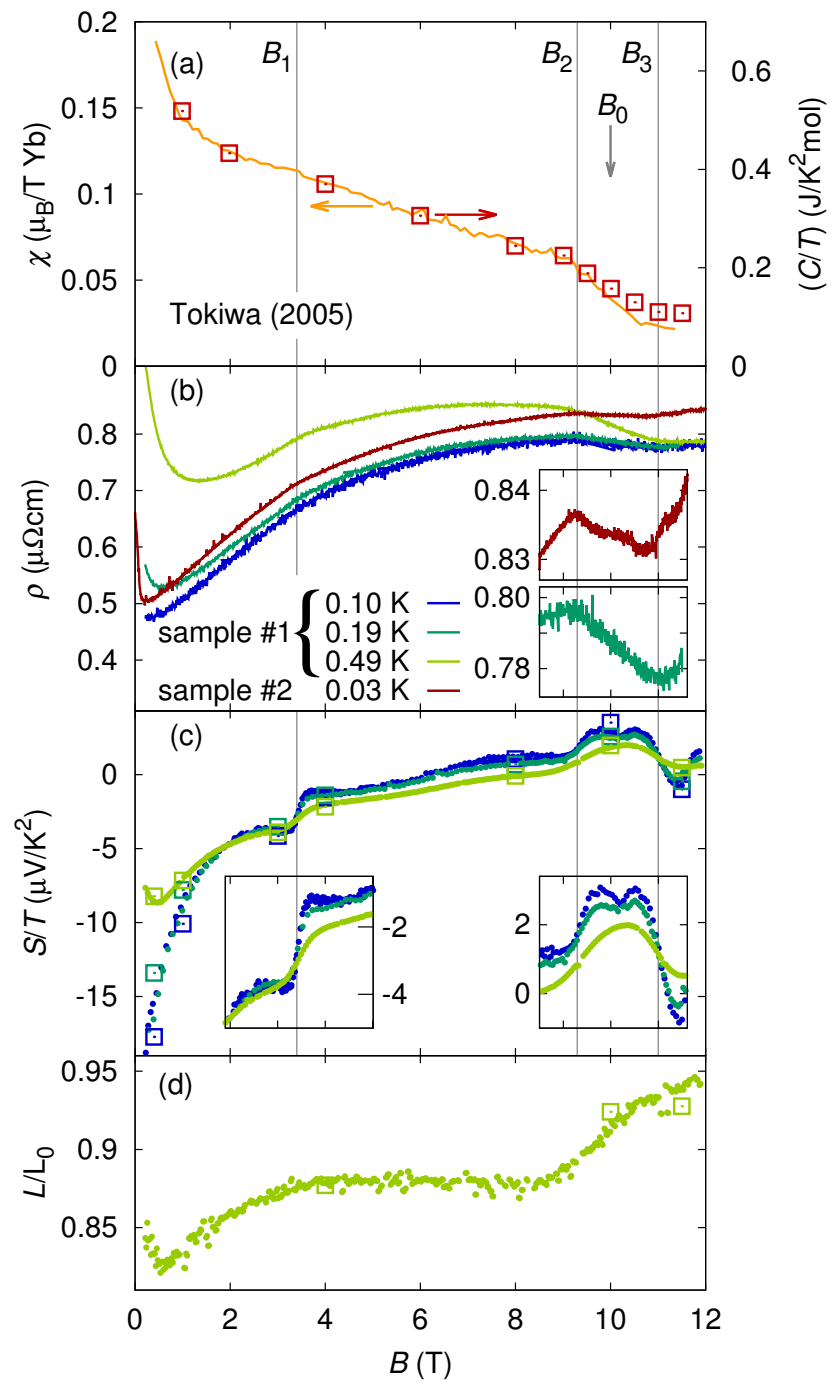

Figure 5.2: Properties of $\mathrm{YbRh}_{2} \mathrm{Si}_{2}$ as a function of magnetic field $B$. Lines and dots denote field sweeps, open squares are extracted from temperature sweeps. (a) The specific heat $C(B) / T$ and the susceptibility $\chi(B)$ taken from Ref. Tok05 show a monotonic decrease with a large slope across $B_{0}$ with kinks at $B_{2}$ and $B_{3}$. (b) $\rho(B)$ shows three kinks at $B_{1}=3.4 \mathrm{~T}, B_{2}=9.3 \mathrm{~T}$, and $B_{3}=11.0 \mathrm{~T}$. Insets: enlarged view for $0.03 \mathrm{~K}$ and $0.19 \mathrm{~K}$. (c) $S(B) / T$ of sample $\# 1$ at the same temperatures as in (b). The complex behaviour with several zero crossings reflects the multi-band character of $\mathrm{YbRh}_{2} \mathrm{Si}_{2}$. All three transitions are characterized by pronounced steps (insets: enlarged view). (d) The Lorenz ratio $L(B) / \mathrm{L}_{0}$ at $0.49 \mathrm{~K}$ is below unity with a minimum at about $0.5 \mathrm{~T}$. 


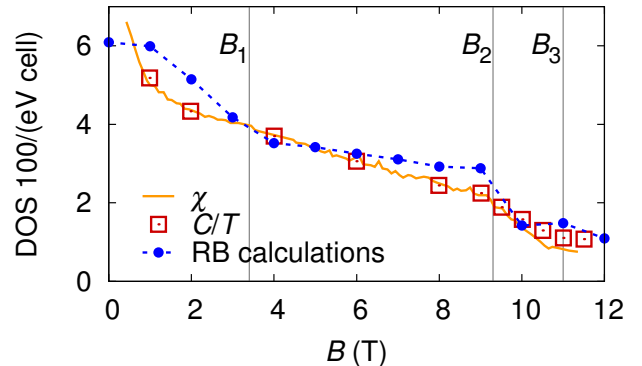

Figure 5.3: Comparison of the field-dependent DOS from experiment Tok05 and from RB calculations Zwi11. The DOS extracted from the specific heat $C / T$ (squares) Zwil1 fits nicely to the predictions of the RB calculations (dots). The dashed line is a guide to the eye. Since the susceptibility $\chi$ (solid line) follows the specific heat, we also included these data here and scaled them accordingly.

to $100 \mathrm{~mJ} / \mathrm{molK}^{2}$ Tok04. It is therefore unlikely that either a single Lifshitz transition Rou08 or a sudden localization of the $f$ electrons Tok04, Tok05, Geg06] is - on its own-a sufficient model to describe this behaviour. Hence, a theory of the field evolution should include not only the Kondo effect to describe de-renormalization processes, but also the specific correlated band structure to reveal topological transitions.

The renormalized-band method is a theoretical approach, which combines material-specific $a b$ initio calculations with phenomenological parameters to account for the strong correlations at $T=0$. The zero-field results are obtained by including, e.g., the experimentally determined crystal electric field scheme and the effective mass from the specific heat. The properties of the quasiparticles in finite magnetic field are then determined using the singleimpurity Anderson model calculated by means of the numerical renormalization group. Details can be found in Ref. Zwi11, Zwi92.

The previously calculated field dependence of the DOS at the Fermi energy by G. Zwicknagl using the RB method was able to quantitatively describe the field evolution of the specific heat (Figure 5.3] [Zwi11]. Therefore, G. Zwicknagl extended these calculations focused on the detailed development of the Fermi surfaces and the energy-dependent DOS in finite magnetic field. She used a tight $k$ mesh in zero field of 8125 points in the irreducible wedge to resolve changes in the isoenergy surfaces. In finite fields she used $405 k$ points.

Figure 5.4(a) shows the calculated zero-field quasiparticle DOS $N(\epsilon)$. Below the Fermi energy, a van-Hove-type singularity is clearly visible. This structure is caused by the anisotropic hybridization of the $4 f$ states with the conduction bands, due to the highly anisotropic crystal electric field ground state. Figure 5.4(b) displays the corresponding isoenergy surfaces of the most important Fermi-surface sheets with $f$ character - the so-called "doughnut"(top) and the "jungle gym" (bottom), respectively. Scanning through $N(\epsilon)$ within the displayed energy range, the calculations predict the four colour-coded regimes characterized by different topologies of the isoenergy surfaces, separated by Lifshitz transitions.

In a magnetic field, the $4 f$ states are split which, in turn, leads to a Zeeman splitting of the quasiparticle bands. The relative shifts of the quasiparticle bands are enhanced by a field-dependent Sommerfeld-Wilson ratio, which reflects the local many-body effects. As a consequence of the band splitting, also the DOS spin-splits into a minority and a majority branch in magnetic field: one spin part moves through the Fermi energy, the other moves quickly away from it (see inset Figure 5.4 (a)). They also do not shift rigidly in field but the amplitude of the coherence peak, i.e., the van-Hove singularity, decreases continuously, mainly because of the weakening of the Kondo effect (Figure 5.4(c)). Nevertheless, the characteristics of the band structure are not changed. Therefore, we expect the energy 


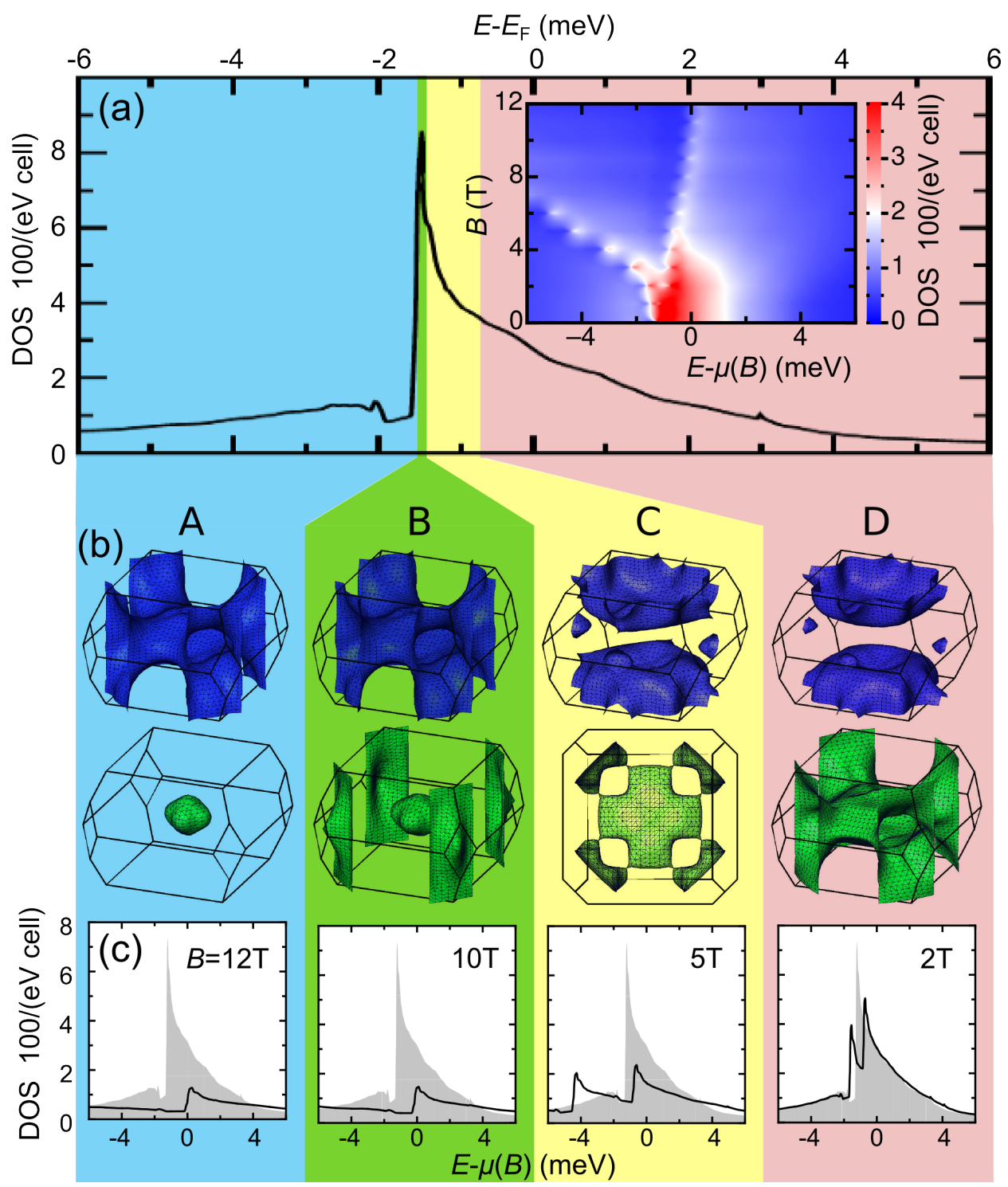

Figure 5.4: Isoenergy surfaces for $B=0$ and the quasiparticle DOS development in finite field calculated using the RB method. The zero-field DOS in (a) is divided into four regions (blue (A), green (B), yellow (C), red (D)) distinguished by different topologies of the isoenergy surfaces shown in (b). In the yellow region (C), we show in plan view the "jungle gym" Fermi-surface sheet exactly at the topological transition between B and C. (c) illustrates the magnetic-field evolution of the DOS for selected fields, with the zero-field DOS in grey for comparison. Inset in (a): Energy-field map of the DOS interpolated in $1 \mathrm{~T}$ steps. One can assign the four energy regions and their isoenergy surfaces in (a) and (b) to the four field ranges and their Fermi surfaces separated by $\widetilde{B}_{1}, \widetilde{B}_{2}$, and $\widetilde{B}_{3}$. 
evolution of the isoenergy surfaces in Figure 5.4(b) to be the same as the field evolution of the Fermi surface. This implies that the Fermi surface of the majority band stays in the red (D) regime, while the Fermi-surface topology of the minority band changes from the red (D) type through yellow (C) and green (B) to the blue one (A).

One can take advantage of the similar shape of the zero- and finite-field DOS and assign the energy of a topology change (in zero field) to a magnetic field where the corresponding feature in the DOS crosses $\epsilon_{\mathrm{F}}$ : The transition from the blue (A) to the green (B) regime corresponds to a kink in the DOS which reaches $\epsilon_{\mathrm{F}}$ for $\widetilde{B}_{3}=(11 \pm 1) \mathrm{T}$. Similarly, one obtains $\widetilde{B}_{2}=(9 \pm 1) \mathrm{T}$ for the second transition (green $(\mathrm{B})-$ yellow $(\mathrm{C})$ ). The difference $\widetilde{B}_{3}-\widetilde{B}_{2}$ fits to the linear shift of $0.1 \mathrm{meV} / \mathrm{T}$ (inset Figure $5.4(\mathrm{a})$ ), which in turn matches the ESR $g$-factor of 3.5 Sch09a (applying $\widetilde{\epsilon}=g \mu_{B} B / 2$ ). We will return to this linear shift in Section 5.6 when we analyse the transport data using the Mott formula. One can use the shift to estimate the field corresponding to the weak third transition from yellow (C) to red $(\mathrm{D})$ to $\widetilde{B}_{1}=(2.5 \pm 1) \mathrm{T}$. These fields, extracted from the calculations, are in excellent agreement with the transitions $B_{1}, B_{2}$, and $B_{3}$ found experimentally. This proves that $B_{1}$, $B_{2}$, and $B_{3}$ correspond to three Lifshitz transitions of the types illustrated in Figure 5.4(b).

At the topological transitions $B_{1}, B_{2}$, and $B_{3}$, we also observe abrupt changes of the density of states $N\left(\epsilon_{\mathrm{F}}\right)$ and the effective mass $m^{*}$ in our calculations. Especially for $B_{2}$ and $B_{3}$ these changes are also visible experimentally in the specific heat and susceptibility (Figure 5.3 . We were also able to detect a thermodynamic response at $B_{1}$ in the second derivative of the magnetostriction (see Figure 5.8 in Section 5.6, where we discuss the Mott formula). These abrupt changes contradict results of the single-impurity Kondo model: Numerical renormalization group calculations predict a continuous decrease of the effective mass $m^{*}$ as we suppress the on-site Kondo effect by a magnetic field [Hew04, Hew06, Bau07; Pet06]. Experiments on diluted $4 f$ alloys, which are the experimental realization of single-ion Kondo systems, show exactly this behaviour Sam76]. Therefore, the single-impurity model alone cannot account for the observations reported above. The features at $B_{1}, B_{2}$, and $B_{3}$ have to be attributed to coherence effects arising from the periodic arrangement of the Kondo ions in a lattice. These coherence effects are captured by our RB calculations. Hence, our results demonstrate that the magnetic-field evolution of $\mathrm{YbRh}_{2} \mathrm{Si}_{2}$ is a combination of Lifshitz transitions due to coherence effects in the Kondo lattice superimposed on a smooth suppression of the Kondo effect.

\subsection{Rigid-band-shift Models for the Field-induced Transitions in $\mathrm{CeRu}_{2} \mathrm{Si}_{2}$ and $\mathrm{YbRh}_{2} \mathrm{Si}_{2}$}

In the previous two sections, we presented our experimental results on the two compounds $\mathrm{CeRu}_{2} \mathrm{Si}_{2}$ and $\mathrm{YbRh}_{2} \mathrm{Si}_{2}$ together with an introduction into the most common and debated interpretations of their high-field transitions from literature. For $\mathrm{YbRh}_{2} \mathrm{Si}_{2}$, we could compare our and previous experimental results with predictions from RB calculations. They account for all observations in thermodynamics and transport and can successfully explain the behaviour of $\mathrm{YbRh}_{2} \mathrm{Si}_{2}$ as a combination of Lifshitz transitions and a smooth suppression of the Kondo effect. Such calculations are demanding and do not exist for $\mathrm{CeRu}_{2} \mathrm{Si}_{2}$ at the moment. Therefore, we want to compare our experiments with more simple rigid-band models. In the case of $\mathrm{YbRh}_{2} \mathrm{Si}_{2}$, we profit from the results of the $\mathrm{RB}$ calculations and 
can illustrate to which extend these models can be applied to the Lifshitz transitions. For the case of $\mathrm{CeRu}_{2} \mathrm{Si}_{2}$, we can demonstrate that the thermopower, as well as other transport properties Dao06a], are compatible with a Lifshitz transition, but that none of our rigid-band models alone can account for both transport and thermodynamic features at the MMT.

The rigid-band models that we will consider in the following are related to suggestions from previous studies. The first one is a "Lifshitz model", which was proposed for $\mathrm{CeRu}_{2} \mathrm{Si}_{2}$ in Ref. Dao06a and for $\mathrm{YbRh}_{2} \mathrm{Si}_{2}$ in Ref. Rou08. The second model is the "peak model", where $\epsilon_{\mathrm{F}}$ is driven through a sharp peak in the DOS, which was suggested for $\mathrm{CeRu}_{2} \mathrm{Si}_{2}$ in Ref. Aok98.

\subsubsection{Transport Signatures}

We will first compare the transport signatures of the models with the observed changes in our materials at the different transitions in Figure 5.5. For the Lifshitz model, the signatures of the thermopower and the conductivity are calculated e.g. in Bla94; Var89 and sketched in Figure 5.5(a),(f) (see also Section 2.5 for an introduction). Both quantities are shown for a transition from region II to region I in the notation of [Bla94]. The thermopower is sketched for hole carriers and either for the case of a neck formation or a void disappearance. For the conductivity, the type of the charge carriers does not matter, but the type of transition is important here and we chose a neck formation. The peak model shows a slight monotonic decrease in the conductivity and a symmetric peak in the thermopower (not shown) Pfa12a. Since the signatures of the peak model are not able to explain our observations in transport for any of the four transitions, we restrict the following discussion of Figure 5.5 to the Lifshitz model. For the electrical transport data we chose $\sigma=1 / \rho$ for simplicity. Note that for $B_{1}$, a smooth background has been subtracted for $S$ and $\sigma$, since both vary strongly in this field range due to the quantum critical fluctuations of the QCP at $60 \mathrm{mT}$.

The MMT in $\mathrm{CeRu}_{2} \mathrm{Si}_{2}$ and the transition at $B_{1}$ in $\mathrm{YbRh}_{2} \mathrm{Si}_{2}$ show clear similarities to the Lifshitz model. In both cases, the asymmetric minimum in the thermopower, slightly left of the transition, points towards a transition from region II to region I for a Fermi surface, which has hole character. The sign of the singular contribution to the conductivity indicates a neck formation. A change in the dHvA frequencies was indeed observed for the hole-like Fermi surface across the MMT in $\mathrm{CeRu}_{2} \mathrm{Si}_{2}$ Aok93. The low field of $B_{1}=3.4 \mathrm{~T}$ of $\mathrm{YbRh}_{2} \mathrm{Si}_{2}$ is, unfortunately, not accessible by quantum oscillations. However, the frequencies of the "doughnut" hole-like Fermi surface were observed to change across $B_{2}$ and $B_{3}$ Rou08]. As we saw in the last Section 5.4 .3 from the RB structure calculations, both hole-like Fermi surfaces of $\mathrm{YbRh}_{2} \mathrm{Si}_{2}$, the "doughnut" and the "jungle gym", undergo topological transitions at $B_{1}, B_{2}$, and $B_{3}$ (see Figure 5.4). At $B_{1}$, the RB calculations predict that a hole appears in the "jungle gym". This corresponds to a ring of Fermi surface points, which becomes critical. This is a very special case of a neck transition and not covered by the calculations shown in Figure 5.5 and 5.6 Bla94.

The transitions at $B_{2}$ and $B_{3}$ in $\mathrm{YbRh}_{2} \mathrm{Si}_{2}$ look different compared to the Lifshitz model. Unfortunately, the two fields lie too close together in order to clearly separate the transitions especially in thermopower. Additionally, the field range of our measurement is with $12 \mathrm{~T}$ too small to estimate the background contribution. However, one can identify kinks in $\sigma$ at the two transition fields and the thermopower shows an asymmetric signature at $B_{2}$, while it seems point symmetric at $B_{3}$. At $B_{2}$, the $\mathrm{RB}$ calculations predict a neck formation in 


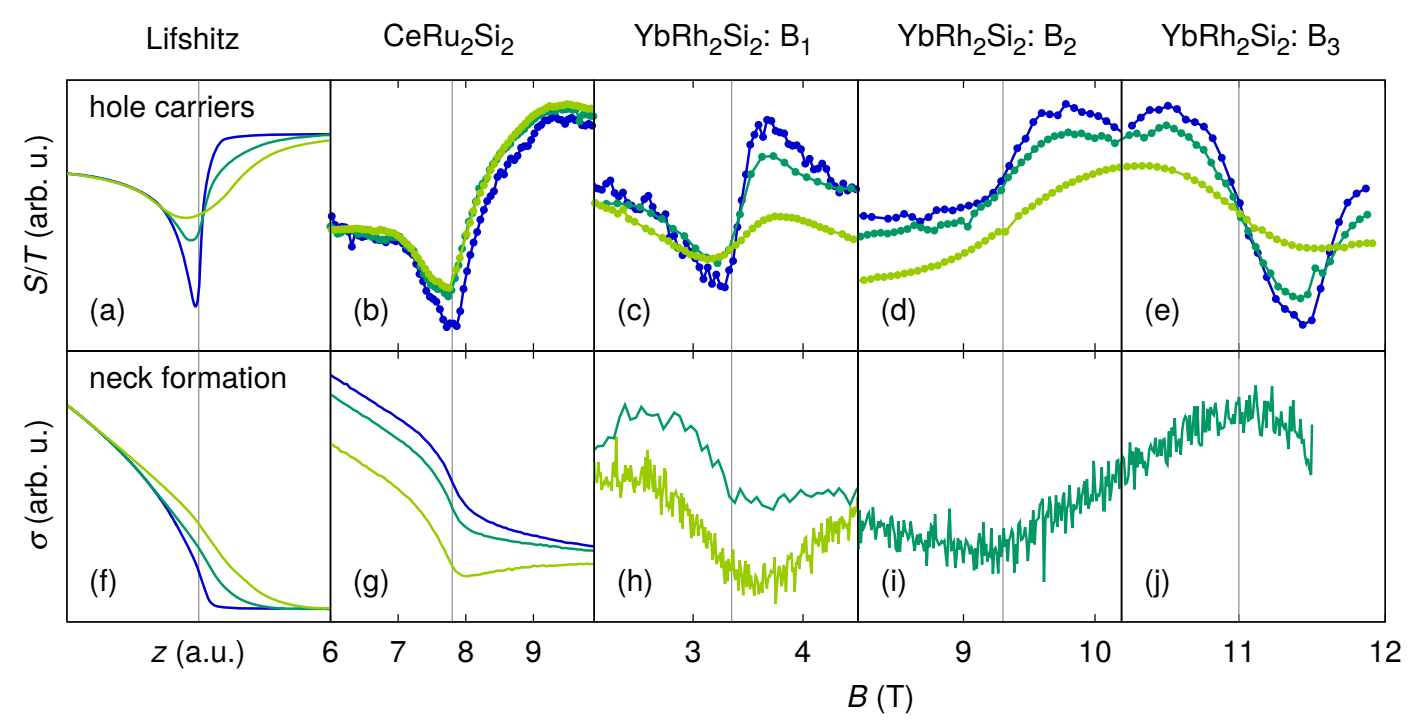

Figure 5.5: Transport signatures of a Lifshitz model versus data. (a) and (f) show the calculated response of the thermopower and the conductivity for a Lifshitz model for three different temperatures (after [Bla94]). We use here the same parameters as in Figure 2.8 of Section 2.5. They are compared with the experimental signatures across the MMT in $\mathrm{CeRu}_{2} \mathrm{Si}_{2}$ (b,g) and the three transitions in $\mathrm{YbRh}_{2} \mathrm{Si}_{2}$ (c-e,h-j). We use the same data already presented in the Figures 5.1 and 5.2 The calculated thermopower is shown for hole-like carriers for a transition from region II to region I. The conductivity is independent of carrier type but is shown here for a neck formation. Note that for (c) and (h) a background was subtracted, which was estimated by a power-law fit including data points between $0.5 \mathrm{~T}<B<2.5 \mathrm{~T}$ and $4.5 \mathrm{~T}<B<8.0 \mathrm{~T}$. We omit the resistivity data for the lowest temperatures in (h-j) due to an enhanced noise level. The data in (h) at $190 \mathrm{mK}$ (dark green) are averaged. The highest temperature data in $(\mathrm{i}, \mathrm{j})$ are omitted due to strongly smeared transitions. Gray lines indicate the transition fields.

both considered Fermi-surface sheets. This is at least consistent with the kink in $\sigma$ and the asymmetry of $S$. At $B_{3}$, a flat sheet on the "jungle gym" vanishes at the zone boundary according to the RB calculations. Unfortunately, it is not clear from the calculations in which way the band disappears. Two ways are conceivable: a simple void transition (2D case) or a combined neck and void transition (3D case). Both cases will create a response in the thermopower which is difficult to predict: For $2 \mathrm{D}$ in contrast to $3 \mathrm{D}$, it was shown that scattering by a random potential caused by all sorts of impurities and dislocations is equally important as scattering between the two Fermi-surface sheets. This renders general statements about $\sigma$ and $S$ almost impossible for 2D Abl91, Bla92. For the second case, the signatures of a neck transition followed closely by a void transition would simply lie too close together to be separated. All in all, only very elaborate calculations of the transport coefficients using the real band structure from the RB calculations could improve our analysis for $B_{2}$ and $B_{3}$. 


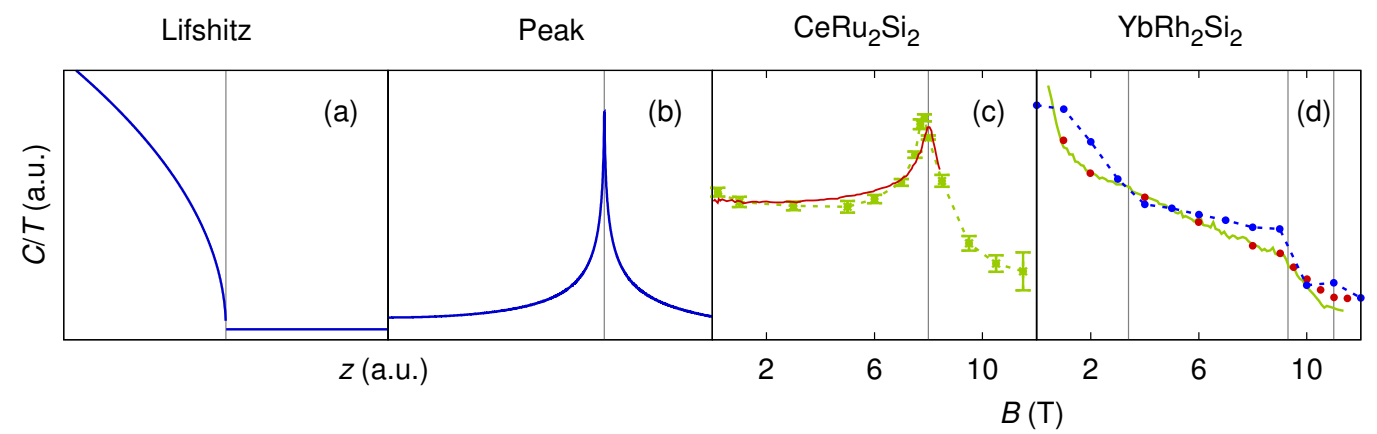

Figure 5.6: Thermodynamic signatures of the Lifshitz and the peak model versus data. The calculated response of the specific heat $C / T$ for $T \rightarrow 0$ in the Lifshitz model (a) and in the peak model (b) are compared with experimental results on $\mathrm{CeRu}_{2} \mathrm{Si}_{2}$ (c) and $\mathrm{YbRh}_{2} \mathrm{Si}_{2}$ (d). For $\mathrm{CeRu}_{2} \mathrm{Si}_{2}$, we show the specific heat from Aok98 (red line) and our data for the $A$ coefficient (green dots). For $\mathrm{YbRh}_{2} \mathrm{Si}_{2}$, we show the specific heat (red dots) and the susceptibility (green line) from Tok05 together with the results from RB structure calculations (blue dotted line) from Zwi11. Gray lines indicate the transition fields.

\subsubsection{Thermodynamic Signatures}

We now turn to the thermodynamic signatures of the transitions in $\mathrm{YbRh}_{2} \mathrm{Si}_{2}$ and $\mathrm{CeRu}_{2} \mathrm{Si}_{2}$. In order to compare them with the predictions from the Lifshitz and the peak model, we plot the specific heat coefficient $C / T$ as well as the susceptibility for $\mathrm{YbRh}_{2} \mathrm{Si}_{2}$ Tok05, and $C / T$ Aok98] as well as the $A$ coefficient for $\mathrm{CeRu}_{2} \mathrm{Si}_{2}$ in Figure 5.6 together with the model calculations. The predictions from the Lifshitz model are again taken from Bla94, Var89 and plotted for the case of a transition from region II to region I as for the transport signatures in Figure 5.5. $C / T$ shows a kink at the transition with a diverging slope on one side. Here, it does not matter which kind of carrier and which type of Lifshitz transition is considered. For the peak model, the specific heat shows a peak at the transition.

While the transport signatures in $\mathrm{CeRu}_{2} \mathrm{Si}_{2}$ can be well described with the Lifshitz model, it fails to account for the specific heat. On the other hand, the peak model can reproduce the peak seen in $C / T$. Hence, either model alone is insufficient to explain the features seen in both transport and thermodynamics. For $\mathrm{YbRh}_{2} \mathrm{Si}_{2}$, the RB calculations proved that $B_{1}$, $B_{2}$, and $B_{3}$ are Lifshitz transitions. Concerning the transport signatures, only $B_{1}$ clearly followed the predictions from the Lifshitz model, however. The specific heat in turn does not show any divergence typical for a Lifshitz transition. Also a rigid shift of the DOS peak calculated with the RB method ( $c f$. Figure 5.4(a)) cannot account for the data [Zwi11. Instead, only the full field-dependent RB calculations shown in Figure 5.6(d) are able to describe the whole field range, in particular the signatures at the transitions.

\subsection{The Mott Formula in $\mathrm{CeRu}_{2} \mathrm{Si}_{2}$ and $\mathrm{YbRh}_{2} \mathrm{Si}_{2}$}

In the following section, we want to investigate the validity of the Mott formula in $\mathrm{CeRu}_{2} \mathrm{Si}_{2}$ and $\mathrm{YbRh}_{2} \mathrm{Si}_{2}$. This formula relates the thermopower with the electrical conductivity and 
is thus a similar transport ratio as the WF law, which we studied in Chapter 4 . Due to the more complicated structure of the Mott formula, it was, however, not studied as extensively as the WF law both experimentally and theoretically. The analysis we present in the following proves a violation of the Mott formula in $\mathrm{CeRu}_{2} \mathrm{Si}_{2}$, but its validity in a wide field region for $\mathrm{YbRh}_{2} \mathrm{Si}_{2}$. This enables us to investigate in detail differences in the scattering mechanisms present in these two compounds in the different magnetic-field regions. The very good agreement of the thermopower and thermodynamic quantities in comparison with an extended version of the Mott formula in $\mathrm{YbRh}_{2} \mathrm{Si}_{2}$ especially around the three transitions corroborates our analysis with the RB structure calculations. At the end of this section, we comment on the $q$ ratio to illuminate the limits of this widely used quantity to interpret thermopower investigations.

\subsubsection{General Approach}

We analyse our data with the help of the Mott formula Mot71 for the diffusion thermopower (cf. Section 2.7.4). It relates the thermopower to the energy derivative of the electrical conductivity $\sigma$

$$
\frac{S}{T}=\left.\frac{\pi^{2} k_{\mathrm{B}}^{2}}{3 e} \frac{\partial \ln \sigma}{\partial \epsilon}\right|_{\epsilon_{\mathrm{F}}} .
$$

The above relation is derived using the Sommerfeld expansion to leading order and the relaxation-time approximation Ash76]. It is also valid for a multi-band case where single contributions to the thermopower are summed by $\sigma S=\sum S_{i} \sigma_{i}$ (cf. Section 2.7.4). Since we cannot access the energy derivative experimentally, we further expand Equation 5.1 and exchange the energy with the field derivative using $\partial B / \partial \epsilon$. Further on, we assume a linear relationship between $\epsilon$ and $B$, which can be written as an effective Zeeman energy $\epsilon=g_{\mathrm{eff}} \mu_{B} B / 2$ :

$$
\frac{S}{T}=\left.\frac{\pi^{2} k_{\mathrm{B}}^{2}}{3 e} \frac{\partial B}{\partial \epsilon} \frac{\partial \ln \sigma}{\partial B}\right|_{\epsilon_{\mathrm{F}}}=\left.\frac{\pi^{2} k_{\mathrm{B}}^{2}}{3 e} \frac{2}{g_{\mathrm{eff}} \mu_{B}} \frac{\partial \ln \sigma}{\partial B}\right|_{\epsilon_{\mathrm{F}}} .
$$

We will use this formula to compare the thermopower with the electrical conductivity for $\mathrm{CeRu}_{2} \mathrm{Si}_{2}$ and $\mathrm{YbRh}_{2} \mathrm{Si}_{2}$ in Section 5.6.3

In a second step, we modify Equation 5.2 using the free-electron picture and insert $\sigma=$ $n e^{2} \tau / m^{*}$ together with $m^{*} \propto N^{2 / 3}$ (in $3 \mathrm{D}$ ) to obtain

$$
\frac{S}{T} \propto \frac{2}{g_{\mathrm{eff}} \mu_{B}}\left(\frac{\partial \ln \tau}{\partial B}-\frac{2}{3} \frac{\partial \ln N}{\partial B}\right)_{\epsilon_{\mathrm{F}}} .
$$

Here, $n$ is the total electron concentration, $N$ the DOS, $m^{*}$ the effective mass, and $\tau$ the scattering time. This splits the thermopower into a scattering part $(\tau)$ and a part representing the band structure $(N)$. We will use Equation 5.3 in Section 5.6.4 to compare the thermopower with the magnetostriction coefficient for $\mathrm{YbRh}_{2} \mathrm{Si}_{2}$.

A very simple form of the Mott formula is the widely used $q$ ratio. This ratio quantifies an empirical relationship between $S / T$ and $\gamma$ in heavy-fermion systems as $T \rightarrow 0$ Beh04:

$$
q=\frac{S}{T} \frac{N_{\mathrm{Av}} e}{\gamma} \approx \pm 1
$$

It is derived from the Mott formula in Equation 5.1 under the assumption of a free electron gas and a scattering time following $\tau=\tau_{0} \epsilon^{\zeta}$. For $\zeta=0$, namely an energy-independent 


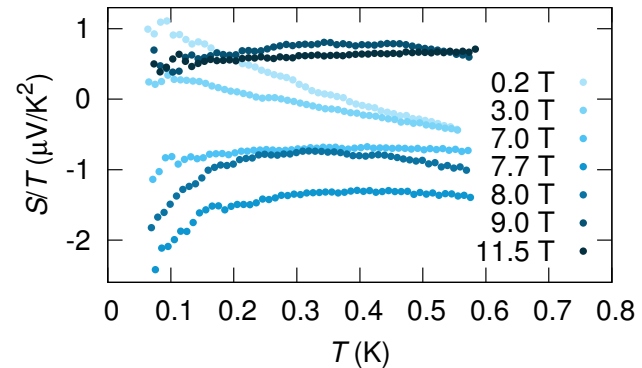

Figure 5.7: Temperature dependence of the thermopower in $\mathrm{CeRu}_{2} \mathrm{Si}_{2}$. Below $0.6 \mathrm{~K}$, the thermopower $S / T$ varies linear with $T$ for small magnetic fields and changes to a constant temperature dependence at higher fields. One expects $S / T=$ const. for a negligible phonon-drag contribution to the thermopower.

scattering time, we obtain $q= \pm 1$, where +1 accounts for electrons, -1 for holes. Here, $N_{\mathrm{Av}}$ is Avogadro's number. We will comment on the $q$ ratio for both compounds in Section 5.6 .5 .

\subsubsection{Phonon Drag}

The Mott formula is only applicable when the phonon-drag contribution to the thermopower is negligible. One expects the diffusion thermopower to be linear in $T$, while the phonon drag contribution usually has a different $T$ dependence, which however varies strongly between different materials Bla76. A negligible phonon-drag contribution within our investigated temperature window has been proven for $\mathrm{YbRh}_{2} \mathrm{Si}_{2}$ Har10a from a linear-in- $T$ behaviour of $S$ in this compound above $0.4 \mathrm{~T}$ and in the reference $\mathrm{LuRh}_{2} \mathrm{Si}_{2}$ for zero field. In $\mathrm{CeRu}_{2} \mathrm{Si}_{2}$, the situation is less clear. While $S / T$ is linear in $T$ for zero field below $500 \mathrm{mK}$, it turns over to $S / T=$ const. only above $8 \mathrm{~T}$ (see Figure 5.7 and Ref. Bou14]). At the same field also the range of the $T^{2}$ behaviour of the resistivity dramatically increases. One expects, however, the largest phonon-drag contribution with a peak around $0.1 \Theta_{\mathrm{D}}$ Bla76, which is above $10 \mathrm{~K}$ Bes85. In this temperature range, the thermopower is completely dominated by a peak due to Kondo scattering [Hae87; Ama89]. The origin of an additional extremum around $1 \mathrm{~K}$ Ama89, Bou14 is unclear.

\subsubsection{Validity in $\mathrm{YbRh}_{2} \mathrm{Si}_{2}$ but not in $\mathrm{CeRu}_{2} \mathrm{Si}_{2}$}

We will start our analysis with the case of $\mathrm{YbRh}_{2} \mathrm{Si}_{2}$ and compare $S / T$ with $\partial \ln \sigma / \partial B$ in Figure 5.8(a). Since the field was applied parallel to the current, we will use $\sigma=1 / \rho$. In the low-field region below $2 \mathrm{~T}$ both curves show a disparate behaviour. However, above $3 \mathrm{~T}$, they can be scaled almost perfectly on top of each other using a $g_{\text {eff }}$ of $16 \pm 1$. The only adjustable parameter leading to this remarkable accuracy of the Mott formula is $g_{\mathrm{eff}}$. An offset is not required. Moreover, the thermopower as well as $\partial \ln \sigma / \partial B$ are independent of sample geometry, thus systematic errors are almost negligible.

The linear energy-field dependence found in our analysis confirms the linear sweep of the van-Hove singularity predicted by the RB calculations presented in Section 5.4.3. However, $g_{\text {eff }}=16$ (corresponding to a $\left.\partial \epsilon / \partial B=0.5 \mathrm{meV} / \mathrm{T}\right)$ differs from $g=3.5(0.1 \mathrm{meV} / \mathrm{T})$ of our calculations. But this is unsurprising, since a rigid-band shift is insufficient to account for the experimental data as we discuss in Section 5.4.3 in detail, where we present our $\mathrm{RB}$ calculations. Unexpectedly, these field-induced changes of the band structure also enter linearly into $g_{\text {eff }}$. 


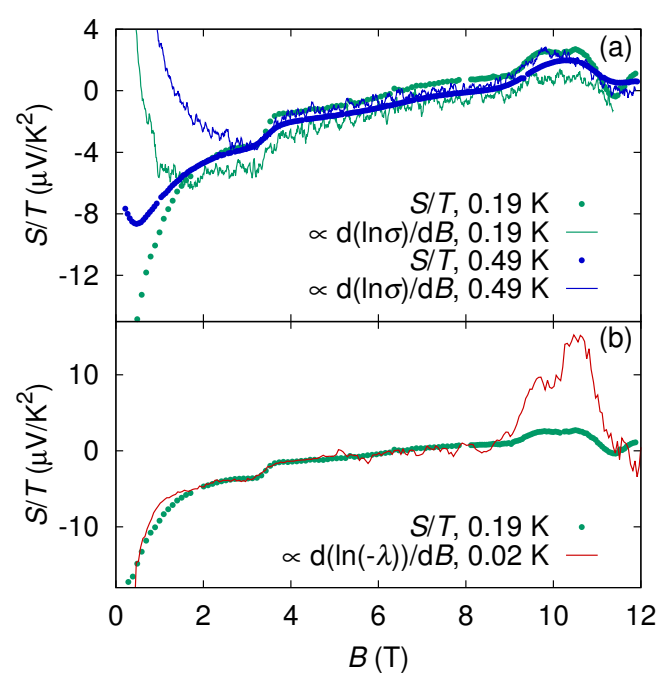

Figure 5.8: Analysis of the thermopower of $\mathrm{YbRh}_{2} \mathrm{Si}_{2}$ with the Mott formula. The measured $S / T$ (dots) is compared with that calculated (lines) from (a) the electrical conductivity using Equation 5.2 and from (b) the magnetostriction coefficient using Equation 5.3 with $\partial \ln N / \partial B \propto \partial \ln \lambda / \partial B$ and $\partial \ln \tau / \partial B=0$. Within the Fermi-liquid regime $(B>2-3 \mathrm{~T})$ all curves show the same overall behaviour. In the representation of the magnetostriction as shown in (b), we are able to prove the presence of the transition $B_{1}$ also in a thermodynamic probe.

We will now turn to $\mathrm{CeRu}_{2} \mathrm{Si}_{2}$. The comparison of our electrical-resistivity and thermopower data according to Equation 5.2 is shown in Figure 5.9. The electrical conductivity is calculated using the data from Dao06a by $\sigma=\rho_{x x} /\left(\rho_{x x}^{2}-\rho_{x y}^{2}\right)$. We use here the notation of Ref. [Dao06a] where $\rho_{x x}$ denotes the longitudinal and $\rho_{x y}$ the transverse magnetoresistivity. Similar results are obtained using our magnetoresistance alone. We choose $g_{\text {eff }}=250$, to reach a reasonable agreement of the average values. However, it is obvious that the modified Mott formula of Equation 5.2 is not valid over the whole field range. There are some similarities, such as the minimum at $B_{\mathrm{MMT}}$ and a shoulder to the left of it. To emphasize this, we show an enlarged view around the MMT in the inset of Figure 5.9 and shifted the curve of $\partial \ln \sigma / \partial B$ by $-0.5 \mu \mathrm{V} / \mathrm{K}^{2}$. However, the relative magnitude of the effects remains different compared to the thermopower. Interestingly, the signature at $1 \mathrm{~T}$ is absent also in the derivative of $\sigma$.

To explain the differences for both materials, we go back to the assumptions made for Equation 5.2 . These are

a) $\epsilon \propto B$

b) $T \ll T_{\mathrm{F}}$

c) $\sigma$, i.e. also $v$ and $\tau$, are analytic functions of $\epsilon$

d) isotropic scattering, $\tau$ only depends on $\epsilon$.

We introduced a) in order to expand the original Mott formula 5.1 towards Equation 5.2 which is suitable for our data analysis. b) and c) originate from the Sommerfeld expansion. While b) is valid for the measurements on both materials, c) can cause problems at Lifshitz transitions. The single relaxation-time approximation is applicable if one assumes d). At very low temperatures, electron-defect scattering and electron-electron umklapp scattering are relevant contributions to momentum relaxation. Scattering on point defects is isotropic, whereas scattering on line defects like dislocations or on surface defects is anisotropic. Umklapp scattering, which is responsible for the Fermi-liquid $T^{2}$ behaviour, is anisotropic due 


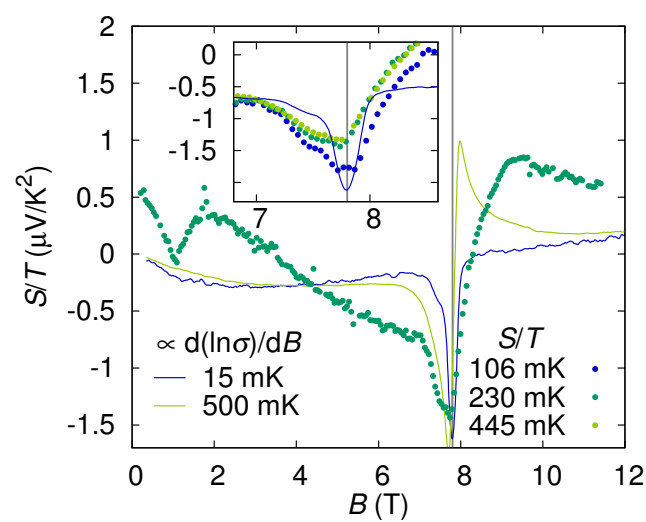

Figure 5.9: Analysis of the thermopower of $\mathrm{CeRu}_{2} \mathrm{Si}_{2}$ with the Mott formula. Following Equation 5.2 we calculated the field derivative of the electrical conductivity $\sigma$ using the data from Dao06a. We used $g_{\text {eff }}=250$. The curves for $\sigma$ and $S$ share some similarities but do not overlap in the whole field range. Inset: Enlargement around $B_{\mathrm{MMT}}$. The curve derived from $\sigma$ is shifted by $-0.5 \mu \mathrm{V} / \mathrm{K}^{2}$ for the inset to illustrate the different development of $S / T$ and $\partial \ln \sigma / \partial B$ across $B_{\mathrm{MMT}}$.

to the strong momentum-space constraints. In this spirit, the validity of Mott's formula is discussed in detail from the theoretical point of view by Buhmann et al. Buh13. On the basis of a simple band structure, they observe severe discrepancies between the thermopower calculated using the Mott formula and the one derived from the Boltzmann equation including the full collision integral taking angular and radial degrees of freedom into account. These discrepancies occur when the umklapp scattering is highly anisotropic, but vanishes at low-enough temperatures, when only isotropic electron-impurity scattering and electronboundary scattering is relevant. However, they survive at a Lifshitz transition even to low temperatures due to a vanishing quasiparticle velocity.

The validity of the Mott formula in $\mathrm{YbRh}_{2} \mathrm{Si}_{2}$ above $2 \mathrm{~T}$ consequently indicates that all scattering processes and in particular electron-electron scattering, which is still present in the investigated temperature range $\left(\rho \propto T^{2}, L / \mathrm{L}_{0}<1\right)$, must be isotropic. Ref. Cus04 shows that $\rho$ in zero field is anisotropic above $20 \mathrm{~K}$ with respect to the current directions $j \perp c$ and $j \| c$. However it becomes isotropic at low $T$. The validity of the Mott formula also at $B_{1}, B_{2}$, and $B_{3}$ is very surprising, since they mark Lifshitz transitions, as we proved in Section 5.4 .3 .

In the low-field region below $2 \mathrm{~T}, S / T$ and $\partial \ln \sigma / \partial B$ show a disparate behaviour. Therefore, either a), c), or d) are violated. First of all, the Fermi-liquid scale $T_{\mathrm{FL}}$ is strongly reduced in this field range: While $T_{\mathrm{FL}} \approx 0.4 \mathrm{~K}$ at $4 \mathrm{~T}$, it is reduced to $0.2 \mathrm{~K}$ at $1 \mathrm{~T}$. These values nicely agree with the increasing range of validity of the Mott formula as we reduce the temperature. In the non-Fermi-liquid regime, which is connected to the quantum critical fluctuations of the field-induced QCP, additional scattering mechanisms can lead to a violation of $\mathrm{d}$ ). Close to a $\mathrm{QCP}$, also the assumption a) with $\epsilon \propto B$ may be wrong.

In the case of $\mathrm{CeRu}_{2} \mathrm{Si}_{2}$, where the Mott formula 5.2 is not valid, again either a), c), or d) may be inadequate assumptions. Away from the MMT, a) and c) are reasonable assumptions. This suggests that the umklapp scattering is anisotropic. Indeed, an anisotropy with respect to the current direction was observed in the magnetoresistance down to $30 \mathrm{mK}$ and also in the field and temperature-dependent thermopower down to $200 \mathrm{mK}$ Bou14. In particular, the violation of the Mott formula above the MMT, where both the resistivity and the thermopower show clear Fermi-liquid-like behaviour in the whole investigated temperature range, demonstrate the sensitivity of the Mott formula to subtle details of scattering mechanisms. 


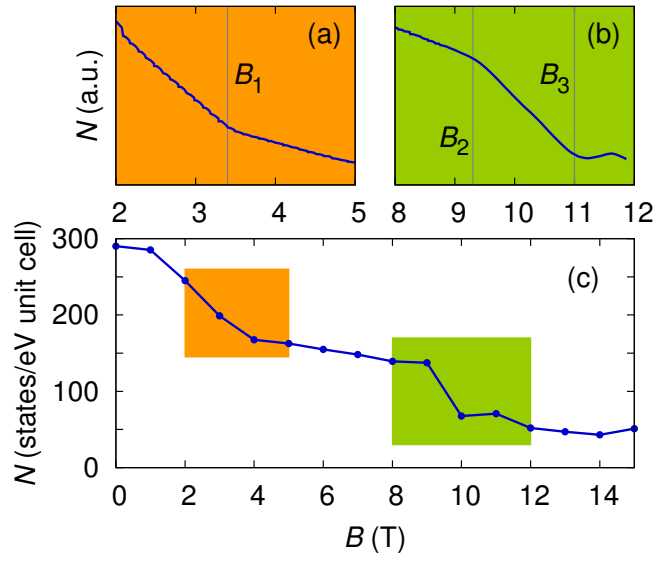

Figure 5.10: Field dependence of the density of states $N$ at the Fermi level $\epsilon_{\mathrm{F}}$. (a) and (b) show the calculated DOS using thermopower data at $0.1 \mathrm{~K}$. For (a) $+S$ and for (b) $-S$ was integrated to match the band-structure calculations from Ref. Zwi11 in (c). All three transition fields $B_{1}, B_{2}$, and $B_{3}$ show up as a kink in the DOS.

Close to $B_{\mathrm{MMT}}$, all three assumptions are certainly problematic: a) The magnetization shows a strong increase around $B_{\mathrm{MMT}}$. A more advanced version of Equation 5.2 with $\epsilon \propto M$ is also not able to achieve a better agreement Pfa12a. c) Since the signatures around $B_{\mathrm{MMT}}$ are compatible with a Lifshitz transition, this assumption may also be violated. d) The Fermi-liquid scale although finite is strongly reduced at $B_{\mathrm{MMT}}$. This gives rise to additional scattering channels, which may be anisotropic.

The above analysis illustrates the ability of the Mott formula to indicate anisotropies in scattering processes. Electron-electron umklapp scattering can become very anisotropic in cases of low-dimensional Fermi surfaces or for small Fermi surfaces, which have a size at the limit where umklapp scattering becomes possible.

\subsubsection{Comparison of Thermopower and Density of States in $\mathrm{YbRh}_{2} \mathrm{Si}_{2}$}

Since the Mott formula works very well in the case of $\mathrm{YbRh}_{2} \mathrm{Si}_{2}$, we will now compare the thermopower with thermodynamic probes using Equation 5.3. Several thermodynamic probes depend upon the DOS, while being independent of scattering effects. We use the linear magnetostriction coefficient $\lambda$, since the data available have a higher resolution than, e.g., specific heat or magnetization. Applying a constant scaling factor, $\partial \ln \lambda / \partial B$ matches $S / T$ in Figure 5.8(b), which implies a power law $\lambda \propto N^{\alpha}$. Only the double hump around $10 \mathrm{~T}$ is more pronounced. Considering the nice agreement at low fields, it is likely that the discrepancy of $S$ and $\sigma$ in this regime is due to anisotropic scattering rather than a failure of $\epsilon \sim B$.

Encouraged by the good qualitative agreement between $S / T$ and $\lambda$ in Figure 5.8 (b), we calculate the field-dependent DOS at $\epsilon_{\mathrm{F}}$ straightforwardly from the thermopower measurements by integrating $S$ over $B$ (ignoring $(\partial \ln \tau / \partial B)$ in Equation 5.3). Importantly, the so obtained DOS (Figure $5.10(\mathrm{a}, \mathrm{b})$ ) matches the features at the transition fields as calculated by the RB method (Figure $5.10(\mathrm{c})$ ): a kink at $B_{1}$ and a step-like decrease between $B_{2}$ and $B_{3}$.

The surprisingly good qualitative agreement in the comparisons of $S$ with $\lambda$ and with the DOS, especially at $B_{1}, B_{2}$, and $B_{3}$, proves again that the origin of all three transitions lies within the correlated band structure of $\mathrm{YbRh}_{2} \mathrm{Si}_{2}$. 


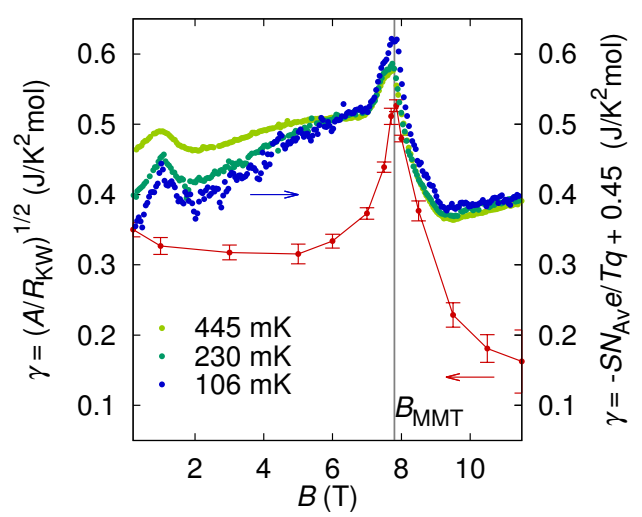

Figure 5.11: The $q$ ratio in $\mathrm{CeRu}_{2} \mathrm{Si}_{2}$. We calculate the electronic specific-heat coefficient, $\gamma$, in two ways. On the left scale we plot $\gamma$ obtained from the Kadowaki-Woods ratio, using the $A$ coefficient of the electrical resistivity in the formula $A / \gamma^{2}=R_{\mathrm{KW}}$. On the right scale we use the $q$ ratio (see text) to scale the thermopower data into units of $\gamma$. We also invert the sign and offset it by $0.45 \mathrm{~J} \mathrm{~mol}^{-1} \mathrm{~K}^{-2}$ to match the left scale at zero field. An offset is justified by the presence of other bands, while the sign inversion is appropriate for heavy-fermion materials. Clearly, the structure in the thermopower is more complex than the signatures in the conductivity and specific heat.

\subsubsection{The $q$ Ratio}

Now, we compare the thermopower with the specific heat using the $q$ ratio as defined in Equation 5.4. We will do that explicitly for $\mathrm{CeRu}_{2} \mathrm{Si}_{2}$. In Figure 5.11 we compare a $\gamma$, which is defined by the $A$ coefficient $\left(\gamma_{A}\right)$ via the Kadowaki-Woods relationship, with another $\gamma$ defined by $S / T$ via the $q$ ratio $\left(\gamma_{S}\right)$. We use $q=-1$. Although the zero-field values do not match (note that $\gamma_{S}$ is shifted), the enhancement of $\gamma_{A}$ at the MMT is indeed similar in magnitude to the enhancement of $\gamma_{S}$. With $q=-1$, we thus interpret the large peak as an enhancement of contributions from a hole-like Fermi surface. This is also in line with the results from the analysis using the "Lifshitz model" in Section 5.5. The natural candidate is the heavy hole band observed by dHvA Tak96, Aok01, Dao06a]. However, the detailed field dependence of $\gamma_{A}$ and $\gamma_{S}$ is very different. The kink in $S / T$ at $B_{\mathrm{MMT}}$ corresponds to the location of the peak in $A$, but the structures around $B_{\mathrm{MMT}}$ in the thermopower are much richer than the thermodynamic ones. This poor agreement is not surprising given the bad results already seen for the previous comparison with Equation 5.2 . The peak at $1 \mathrm{~T}$ is also not correlated with any feature seen in thermodynamic properties.

The same comparison can be done for $\mathrm{YbRh}_{2} \mathrm{Si}_{2}$. We do not show it explicitly here, but the general trend is already obvious from a comparison of $S / T$ and $\gamma=C / T$ in Figure 5.2 in Section 5.4.2. With $q=-1$ (for Yb systems Beh04), there are again some similarities such as the general decrease of $\gamma$ and $q S / T=-S / T$ with increasing magnetic field and the overall magnitude of this decrease seems to be similar in both. But although the Mott formula works exceptionally well in this material even in the very simple form of Equation 5.3 ignoring any contribution from $\tau$, severe differences between $\gamma$ and $S / T$ occur in the details of the field dependence using the q ratio. This is especially pronounced around the transitions $B_{1}, B_{2}$, and $B_{3}$.

Hence, the q ratio can indeed be used to estimate the magnitude of field-driven effects in the thermopower from the specific heat, however, it is too simple to serve as an adequate description of structures in $S(B)$. 


\subsection{Conclusion}

With the help of field-dependent thermal and electrical transport measurements, we studied the magnetic-field evolution of the Kondo-lattice states in $\mathrm{YbRh}_{2} \mathrm{Si}_{2}$ and $\mathrm{CeRu}_{2} \mathrm{Si}_{2}$.

In $\mathrm{YbRh}_{2} \mathrm{Si}_{2}$, previous thermodynamic measurements found a magnetic-field-induced transition at $10 \mathrm{~T}$. With our transport studies, we were able to decompose its structure and detected two transitions at $9.3 \mathrm{~T}$ and $11 \mathrm{~T}$. In addition, another one at $3.4 \mathrm{~T}$ could be resolved. All of them were identified as Lifshitz transitions by a comparison with predictions from detailed RB structure calculations. The agreement of our and previous experiments with $\mathrm{RB}$ calculations implies that the unusual high-field properties of $\mathrm{YbRh}_{2} \mathrm{Si}_{2}$ arise from the interplay of a continuous suppression of the local Kondo effect and coherence effects due to the periodicity of the lattice. The first leads to a reduction of the effective mass and a fielddependent Sommerfeld-Wilson ratio. The latter is responsible for three Lifshitz transitions in the investigated field range. The coherence effects are determined by the symmetry of the $4 f$ wave function characterizing the crystal electric field ground state, which leads to an anisotropic hybridization and a van Hove singularity in the DOS. The excellent agreement between our experimental results and the theoretical predictions demonstrates that $\mathrm{RB}$ calculations are a very suitable approach to describe quasiparticle bands in the Kondo lattice.

The thermal transport properties of $\mathrm{CeRu}_{2} \mathrm{Si}_{2}$ at the MMT are also compatible with a Lifshitz transition, a model proposed by earlier electrical-transport studies. However, a conclusive picture, which can also incorporate the thermodynamic features, is still lacking. With the thermopower we could additionally detect another transition at $1 \mathrm{~T}$, which is not resolved by any other probe.

Generally, the flat bands of Kondo-lattice systems and the reduced energy scales of $T_{\mathrm{K}}$ of the order of $10 \mathrm{~K}$ are very suitable to observe Lifshitz transitions induced by a spin splitting in magnetic field. Our investigation proved that the thermopower measurements are a very sensitive tool to detect such transitions. However, it is also one of the most difficult probes to understand. Hence, we started to systematically compare experimental signatures with rigid-band models. The Lifshitz model was able to account for many transport signatures. To establish it as a reference point to detect Lifshitz transitions, however, a larger systematic study and a better theoretical foundation is required to account for effects of magnetic field, for electronic correlations, and also for other special symmetry cases. The discrepancies between the model calculations and especially thermodynamic data highlights the boundaries of rigid-band models and demonstrates the necessity of more advanced theoretical tools such as the RB method.

Our low-temperature measurements as a function of magnetic field also allowed us to study the validity of Mott's formula. We find an extraordinary good agreement with Mott's formula for $\mathrm{YbRh}_{2} \mathrm{Si}_{2}$ starting at moderate fields above the quantum critical regime. This validity is especially unexpected close to the Lifshitz transitions. In contrast, the Mott formula seems to be violated in $\mathrm{CeRu}_{2} \mathrm{Si}_{2}$ in the whole field range and in $\mathrm{YbRh}_{2} \mathrm{Si}_{2}$ at low fields. While the validity indicates isotropic scattering of electronic quasiparticles, the violation of the Mott formula suggests the presence of anisotropic scattering mechanisms.

The present study will serve as a starting point for a more systematic investigation of Mott's formula and of Lifshitz transitions in correlated electron materials and especially Kondo lattices. Investigations on $\mathrm{YbNi}_{4} \mathrm{P}_{2}$ are currently under way. They seem to reveal at least three transitions in fields up to $12 \mathrm{~T}$, some of which show typical Lifshitz signatures 
in transport properties. One can also find other materials in literature such as $\beta$-YbAlB 4 Mat14, which are promising candidates for such a study. 


\section{6 \\ (O) The Superconducting Order Parameter of $\mathrm{LaPt}_{4} \mathrm{Ge}_{12}$}

Filled skutterudites have attracted much interest due to a remarkable variety of physical properties and ground states. Particular attention has been paid to the superconductors, which range from conventional Bardeen-Cooper-Schrieffer (BCS) types to multiband or unconventional superconductors. Here, we present results on the filled skutterudite $\mathrm{LaPt}_{4} \mathrm{Ge}_{12}$, which becomes superconducting below $T_{c}=8.3 \mathrm{~K}$. While for its Pr-counterpart an unconventional and/or a multiband coupling mechanism is discussed, NMR and photoemission measurements on the La compound suggest conventional $s$-wave superconductivity. However, results on the series $\mathrm{La}_{x} \mathrm{Pr}_{1-x} \mathrm{Pt}_{4} \mathrm{Ge}_{12}$ indicate compatible order parameters for the two stoichiometric end compounds. We performed specific-heat measurements above $2 \mathrm{~K}$ and a detailed temperature, magnetic field, and field-angle-dependent thermal-conductivity study down to temperatures below $100 \mathrm{mK}$. All our results are compatible with a single superconducting $s$-wave gap. The field-angle dependence of the thermal conductivity surprisingly shows a rich oscillatory component. Oscillations below $T_{\mathrm{c}}$ are often connected to the presence of nodes in the gap. The nodeless character of the gap in $\mathrm{LaPt}_{4} \mathrm{Ge}_{12}$ conducted from all other measurements demonstrates that a careful analysis of the field-angle dependence is essential for a reliable determination of the gap symmetry.

\subsection{Introduction and Motivation}

In every superconductor, electrons form Cooper pairs due to an attractive interaction which overcomes the Coulomb repulsion. However, the nature of the attraction can vary considerably for different materials. In most superconductors, the coupling is mediated by phonons, but for others, e.g., magnons are considered to be the driving force. The pairing mechanism is directly related to the symmetry of the superconducting order parameter, which in turn is connected to the symmetry of the superconducting gap. Phonon coupling, e.g., leads to an $s$-wave symmetry of the order parameter, which can be described by the Bardeen-CooperSchrieffer (BCS) theory. From today's perspective, unconventional superconductors which go beyond this scenario are most interesting. Their gaps contain nodes and the coupling in these materials can be mediated by magnons or other more exotic excitations.

To establish the unconventional nature of a superconductor, one often concentrates on proving the existence of nodes in the superconducting gap. By now, a whole fleet of experimental methods has been developed for this purpose. Examples are temperature and magnetic-field-dependent specific-heat and thermal-conductivity measurements, nuclear magnetic resonance (NMR), muon spin rotation $(\mu \mathrm{SR})$, and tunnel-diode oscillator-based measurements. Apart from the existence of nodes, these probes are also able to put some 
more constraints on the symmetry of the order parameter. To determine the exact position of the nodes and thus the exact order parameter, however, directional probes are necessary. An example of such a probe is angle-resolved photoemission spectroscopy, which was successfully used to determine the gap structure of the high- $T_{\mathrm{c}}$ cuprates. However, its energy resolution is insufficient for low- $T_{\mathrm{c}}$ systems and it is a surface sensitive probe. In recent years, field-angle-dependent thermal-conductivity (and also specific heat) measurements were established as an angular-sensitive bulk probe of the superconducting gap (for a review see Mat06.).

The search for and study of unconventional superconductors was triggered by the discovery of heavy-fermion superconductivity [Ste79] and the high-temperature cuprate superconductors Bed86]. By now, many more materials are believed to be unconventional superconductors. In this context, the filled skutterudite compounds $R T_{4} X_{12}(R=$ rare-earth metal, $T=$ transition metal, $X=$ usually a pnictogen) attracted much attention with the discovery of $\mathrm{PrOs}_{4} \mathrm{Sb}_{12}$ Bau02, which is believed to be an unconventional superconductor Aok05. It exhibits exotic properties probably connected to the quadrupole degrees of freedom [Aok05].

The newly discovered $\mathrm{PrPt}_{4} \mathrm{Ge}_{12}$ and $\mathrm{LaPt}_{4} \mathrm{Ge}_{12}$ are skutterudites with a Pt-Ge framework, which show superconductivity at relatively high transition temperatures compared to other skutterudites with $T_{c}=7.9 \mathrm{~K}$ and $8.3 \mathrm{~K}$, respectively Gum08. $\mathrm{PrPt}_{4} \mathrm{Ge}_{12}$ seems to be a good candidate for unconventional superconductivity. It is considered to be a strongcoupling SC from the large specific-heat jump compared to the BCS value Gum08. While a number of investigations revealed multiband superconductivity in this compound Nak12, Cha12, Zha13, there are additionally indications of point nodes from NMR Kan10, specific heat and penetration depth Zha13. $\mu$ SR measurements detected a time-reversal symmetry breaking below $T_{c}$ Mai10.

The evolution of $T_{c}$ across the doping series $\left(\mathrm{La}_{x} \mathrm{Pr}_{1-x}\right) \mathrm{Pt}_{4} \mathrm{Ge}_{12}$ indicates, that the order parameters of the end members $\mathrm{PrPt}_{4} \mathrm{Ge}_{12}$ and $\mathrm{LaPt}_{4} \mathrm{Ge}_{12}$ are compatible Mai10. However, the few investigations on $\mathrm{LaPt}_{4} \mathrm{Ge}_{12}$ point towards a single isotropic gap: the specific-heat jump is only slightly above the BCS value suggesting a weaker coupling than in $\mathrm{PrPt}_{4} \mathrm{Ge}_{12}$ Gum08. NMR and photoelectron-spectroscopy results could be best explained by a single isotropic gap Tod08, Nak12. No time-reversal symmetry breaking is observed in $\mu \mathrm{SR}$ Mai10.

To resolve this discrepancy, it is necessary to shed more light on the superconducting properties of both end members $\mathrm{LaPt}_{4} \mathrm{Ge}_{12}$ and $\mathrm{PrPt}_{4} \mathrm{Ge}_{12}$. Since the single crystals available for both compounds are relatively small for a sufficient geometry factor but still larger for $\mathrm{LaPt}_{4} \mathrm{Ge}_{12}$, we decided to study this compound. To this end, we performed first specific-heat measurements at temperatures down to $0.4 \mathrm{~K}$ to obtain a thermodynamic indicator of the gap structure. We use these results to analyse our detailed thermal-conductivity study. The focus of the thermal-transport measurements was on the low-temperature behaviour below $1 \mathrm{~K}$ and we performed extensive temperature, field-, and field-angle-dependent measurements. Those results are compared with predictions from the BCS theory for conventional superconductors and with predictions for multiband or unconventional superconductors.

The thermal-transport measurements were also used to test and extend the abilities of the "Rotator" set-up. We put special effort to increase the resolution and decrease the required measurement time for the field-angle-dependent measurements by implementing a continuous method (see Chapter 3). 


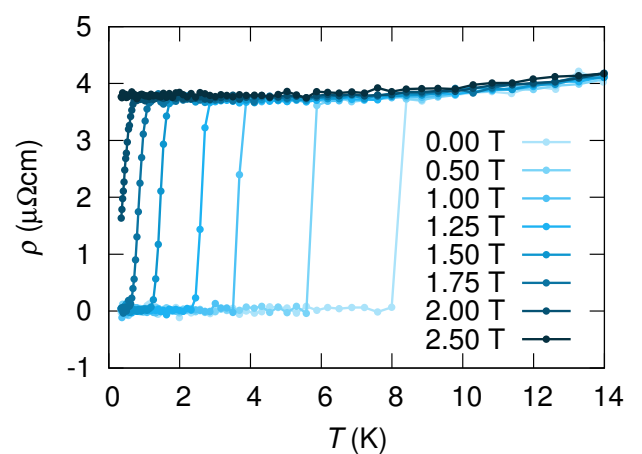

Figure 6.1: Temperature dependence of the resistivity. The sample shows a sharp superconducting transition, which persists to much higher fields than $H_{\mathrm{c} 2}=1.4 \mathrm{~T}$ determined from bulk methods. This can be attributed to surface effects or stoichiometric variations in small parts of the sample. In the normal state, $\rho$ is constant below $6 \mathrm{~K}$ indicating dominant impurity scattering.

\subsection{Experimental Methods}

We investigated three high-quality single crystals of $\mathrm{LaPt}_{4} \mathrm{Ge}_{12}$ from the same batch $1289 \mathrm{~s} 2 \mathrm{k}$. They were grown by applying multistep thermal treatments as descibed in Ref. Zha13. Sample \#1 (1289s2k_4) with a mass of $m=14.7 \mathrm{mg}$ and sample \#2 (1289s2k_6) with $67.2 \mathrm{mg}$ were used for specific-heat measurements in a Quantum Design Physical Property Measurement System (PPMS). On sample \#3 (1289s2k_3) we performed transport measurements. To this end, we cut it first into a rectangular bar along the principal cubic crystal axes with a cross-sectional area of $0.492 \mathrm{~mm}^{2}$ and placed the contacts for the two thermometers along the longest crystal axis separated by $1.08 \mathrm{~mm}$. This arrangement was used for thermal-conductivity measurements in a PPMS above $2 \mathrm{~K}$ and in zero magnetic field. Afterwards, a long plate $\left(1289 \mathrm{~s} 2 \mathrm{k} \_3 \mathrm{a}\right)$ was cut from sample \#3 again along the principal cubic crystal axes with the dimensions $(0.04 \times 0.50 \times 1.92) \mathrm{mm}$. This sample \#3a was used to measure thermal conductivity below $1 \mathrm{~K}$ for magnetic fields 11 of $0 \leq H \leq 2 \mathrm{~T}$ with the current $\boldsymbol{j}$ applied along the same direction as for the PPMS measurement. We used the "Rotator" set-up, where a superconducting split-coil magnet supplies a magnetic field $\boldsymbol{H} \perp \boldsymbol{j}$. To vary the angle of the magnetic field within the plane perpendicular to $\boldsymbol{j}$, the magnet is mechanically rotated around the sample. Sample \#3a was also used to measure the d.c. resistivity between $0.3 \mathrm{~K}<T<20 \mathrm{~K}$ with the same contacts as for the thermal transport.

\subsection{Resistivity}

In Figure 6.1, we present the resistivity $\rho(T)$ of $\mathrm{LaPt}_{4} \mathrm{Ge}_{12}$. We observe a sharp superconducting transition at $8 \mathrm{~K}$. It is suppressed with increasing magnetic field and broadens only weakly compared to zero field. However, superconductivity is still present at $2 \mathrm{~T}$, which is well above the reported critical field of $1.6 \mathrm{~T}$ Gum08 and above the one obtained from specific heat and thermal conductivity of $1.4 \mathrm{~T}$ (see below). Only a field of $2.5 \mathrm{~T}$ pushes the superconducting transition below our measurement limit of $300 \mathrm{mK}$. This effect is frequently observed also in other superconductors and can be attributed for example to a superconducting surface layer with different physical properties compared to the bulk, or to

\footnotetext{
${ }^{1} \mathrm{As}$ it is common for superconductors, we will use the symbol $H$ for the magnetic field instead of $B$ in this chapter. However, we will stick to the more illustrative unit Tesla.
} 

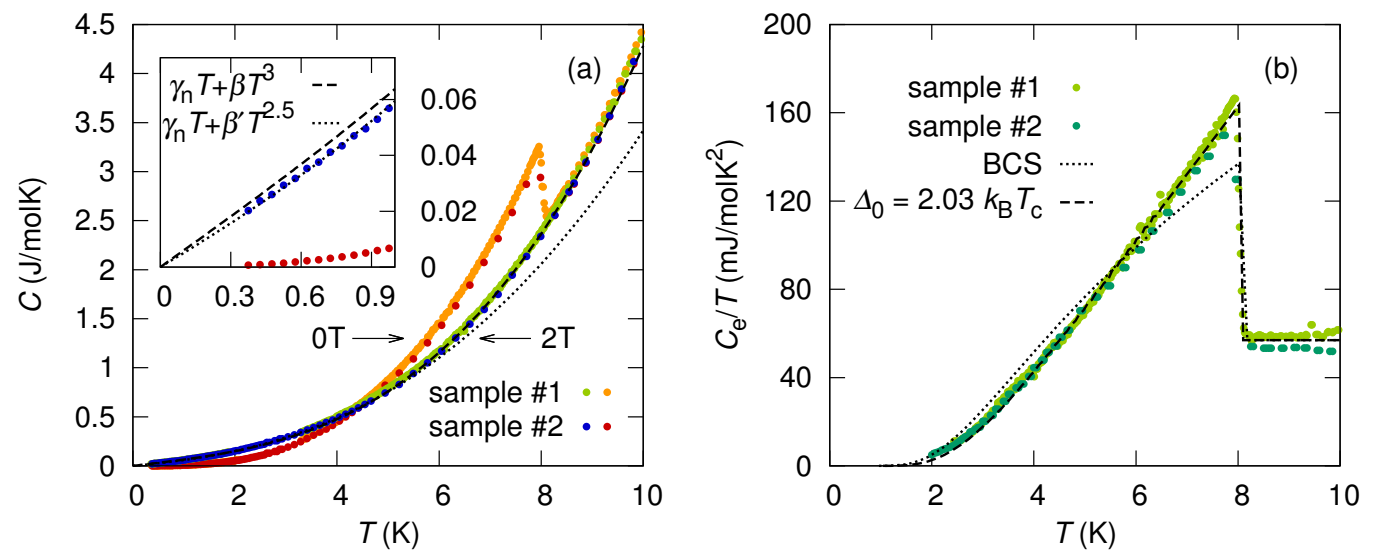

Figure 6.2: Temperature dependence of the specific heat. (a) At zero field, $C$ shows a superconducting transition at $8 \mathrm{~K}$, which is absent at $2 \mathrm{~T}>H_{\mathrm{c} 2}$. A fit of the results at $2 \mathrm{~T}$ with a Debye model falls on top of the data, except below $4 \mathrm{~K}$, where the phonon contribution follows $\beta^{\prime} T^{2.5}$ (see inset). From (a), the electronic contribution $C_{\mathrm{e}}$ to the zero-field curve can be calculated (b). It follows a BCS behaviour with $\Delta_{0}=2.03 k_{\mathrm{B}} T_{\mathrm{c}}$. Both samples show the same results.

superconducting impurity phases perhaps due to stoichiometric variations, which short-cut the sample.

In the normal state, $\rho(T)$ is almost constant below $6 \mathrm{~K}$ with a residual resistivity of $\rho_{0}=3.8 \mu \Omega \mathrm{cm}$ indicating dominant impurity scattering. Furthermore, the magnetic-field dependence is almost negligible below $20 \mathrm{~K}$. The residual resistivity ratio (RRR) of the crystal is $\mathrm{RRR}=\rho(300 \mathrm{~K}) / \rho(0.3 \mathrm{~K})=17$.

\subsection{Specific Heat}

We measured the specific heat $C(T)$ of $\mathrm{LaPt}_{4} \mathrm{Ge}_{12}$ at zero field and in a field of $2 \mathrm{~T}$ above the critical field (Figure 6.2(a)). The latter is used as an estimate of the phonon contribution. Since the resistivity shows a much higher $H_{\mathrm{c} 2}$, we also performed measurements at $2.5 \mathrm{~T}$, which turned out to be identical to the results at $2.0 \mathrm{~T}$. To obtain the electronic contribution to the specific heat $C_{e}$ at zero field, the results at $2 \mathrm{~T}$ are subtracted from the zero-field data except for a constant normal-state electronic contribution $\gamma_{\mathrm{n}}=C_{\mathrm{n}} / T=$ const., which is determined by a Debye fit,

$$
C=\gamma_{\mathrm{n}} T+\beta T^{3},
$$

to $C(2 \mathrm{~T})$ between $0.3 \mathrm{~K}<T<10 \mathrm{~K}$, which is also plotted in Figure 6.2(a). From the fit we obtain $\gamma_{\mathrm{n}}=56 \mathrm{~mJ} / \mathrm{molK}^{2}$ and $\beta=3.7 \mathrm{~mJ} / \mathrm{molK}^{4}$. The fit can describe the data in the whole temperature range well, only below $4 \mathrm{~K}$ there are small deviations and the phonon contribution follows $\beta^{\prime} T^{2.5}$. This deviation is negligible for the following analysis, but becomes important in Section 6.5 where we discuss the thermal conductivity. The result for the electronic contribution to $C(0 \mathrm{~T})$ is shown in Figure 6.2(b) as the specific heat coefficient $\gamma(T)=C_{\mathrm{e}} / T$. Unfortunately, the low-temperature data are too noisy to analyse and we restrict the following discussion of $C_{\mathrm{e}} / T$ to $T>2 \mathrm{~K}$. 

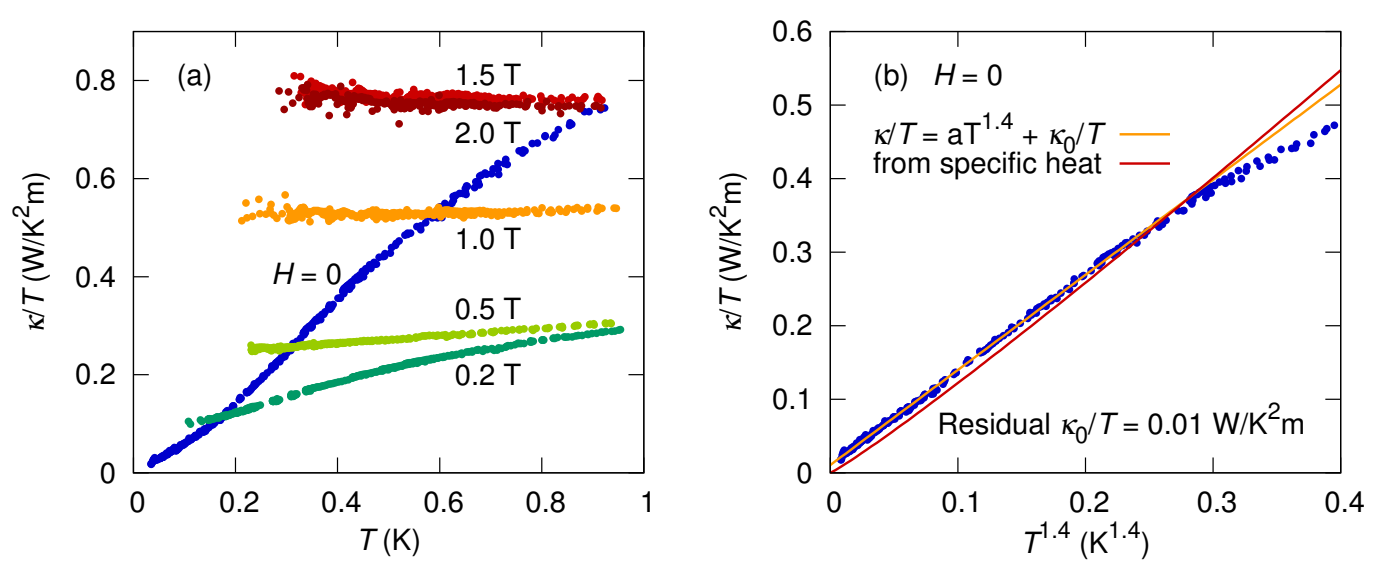

Figure 6.3: Temperature dependence of the thermal conductivity below $1 \mathrm{~K}$. The zero-field thermal conductivity from (a) can be fitted with a power law $\kappa / T \propto T^{1.4}$ (yellow) shown in (b) leading to a residual term of $\kappa / T(T \rightarrow 0)=0.01 \mathrm{~W} / \mathrm{K}^{2} \mathrm{~m}$. A second fit (red) with Equation 6.2 using the specific-heat results indicates a dominant phonon contribution scattered by boundaries (see text). Applying a magnetic field leads to an almost constant $\kappa / T$ above $0.5 \mathrm{~T}$ with parallel shifts for increasing field.

The specific heat exhibits a very sharp superconducting transition at $8.0 \mathrm{~K}$, which is an indication of the good quality of the sample. The jump is higher than expected from the predictions of the weak-coupling BCS model $\left(\Delta_{0}=1.76 k_{\mathrm{B}} T_{\mathrm{c}}\right)$, which cannot reproduce our data. However, we are able to describe $C_{\mathrm{e}} / T$ adjusting the gap to $\Delta_{0}=2.03 k_{\mathrm{B}} T_{\mathrm{c}}$. This value is in agreement with the results from photoelectron spectroscopy: $\Delta_{0}=1.95 k_{\mathrm{B}} T_{c}$ [Nak12], and NMR: $\Delta_{0}=1.92 k_{\mathrm{B}} T_{\mathrm{c}}$ Kan10.

Band-structure calculations by H. Rosner showed, that several bands cross the Fermi energy ( $c f$. Figure 6.6), which opens up the possibility of multiband superconductivity. If there are two gaps, as was observed in the case of $\mathrm{PrPt}_{4} \mathrm{Ge}_{12}$, the specific heat can be described as a weighted sum of the contributions from both gaps within the two-gap $\alpha$ model ( $c f$. Section 2.6.3). Hence, both gaps can have either almost the same size $\Delta_{0}$ or the weighting factor of the second gap is small compared to its size. In the latter case, its contribution to the specific heat stays tiny and only shows up below $2 \mathrm{~K}$. The question whether there is a second gap may be solved by measurements on the substitution series $\left(\mathrm{La}_{x} \operatorname{Pr}_{1-x}\right) \mathrm{Pt}_{4} \mathrm{Ge}_{12}$.

\subsection{Thermal Conductivity}

\subsubsection{T Dependence of the Thermal Conductivity}

Figure 6.3 (a) shows the temperature dependence of the thermal conductivity $\kappa(T)$ below $1 \mathrm{~K}$ in zero magnetic field and for different finite fields. The measurements were performed on warming. For finite magnetic fields they show no difference between field- and zero-fieldcooling. While for $H=0, \kappa / T$ increases with increasing temperature, it rapidly approaches 
a behaviour $\kappa / T=$ const. for $H \geq 0.5 \mathrm{~T}$.

To gain information about the superconducting gap structure, we analyse the zero-field curve in more detail. Figure 6.3(b) shows a power-law fit $\kappa(T) / T=\kappa_{0} / T+b T^{a}$ to our data below $T=0.4 \mathrm{~K}$.

From the fit, we obtain a residual term $\kappa_{0} / T=0.01 \mathrm{~W} / \mathrm{K}^{2} \mathrm{~m}$, which is of the order of our measurement uncertainty. A sizeable residual term is expected for a nodal SC Gra96; Dur00 Sha09 due to pair-breaking impurities. Our observed $\kappa_{0} / T$ is small compared to these expectations and experimental results for unconventional superconductors Wat04; Pro02. Hence, this result points towards a superconductor with a finite gap everywhere on the Fermi surface.

The exponent we obtain from the power law fit is $a=1.4$. In an $s$-wave superconductor, the low-temperature thermal conductivity is expected to be entirely due to phonons. With the relation

$$
\kappa_{\mathrm{p}}=\frac{1}{3} C_{\mathrm{p}} v_{\mathrm{p}} l_{\mathrm{p}}
$$

one can estimate the phonon contribution $\kappa_{\mathrm{p}}$ from the specific heat $C_{\mathrm{p}}$ and the mean free path $l_{\mathrm{p}} . C_{\mathrm{p}}=\beta T^{3}$ is the phonon specific heat from a Debye model determined above. The velocity $v_{\mathrm{p}}$ can be calculated from $C_{\mathrm{p}}$ using

$$
\beta T^{3}=\frac{12 \pi^{4}}{5} N k_{B} \frac{T^{3}}{\Theta^{3}}, \quad v_{\mathrm{p}}=\frac{k_{B} \Theta}{\hbar}\left(\frac{V}{6 \pi^{2} N}\right)^{1 / 3}
$$

where $\Theta$ is the Debye temperature, $V=7.25 \cdot 10^{-9} \mathrm{~m}^{3}$ is the crystal volume of sample \#2 and $N=17 m N_{\text {Av }} / M=3.84 \cdot 10^{20}$ is the number of atoms in the crystal. This leads to $\Theta=208 \mathrm{~K}$ and $v_{\mathrm{p}}=1860 \mathrm{~m} / \mathrm{s}$. However, the experimental specific-heat data on $\mathrm{LaPt}_{4} \mathrm{Ge}_{12}$ follow only approximately a $T^{3}$ dependence, but can be better described by a $T^{2.5}$ law below $4 \mathrm{~K}$ (see inset of Figure $6.2(\mathrm{a}))$. Interestingly, the thermal conductivity follows a $T^{2.4}$ dependence at low $T$, which is close to the $T$ dependence of $C_{\mathrm{p}}$. The almost identical exponents hint towards a scenario, where phonons scattered on boundaries are the main contribution to $\kappa$ at low $T$. A fit of the thermal conductivity with an adjusted power law $C_{\mathrm{p}}=\beta^{\prime} T^{2.5}$, with the velocity $v_{\mathrm{p}}$, and with the free parameter $l_{\mathrm{p}}$ is shown in Figure 6.3 (b). It leads to a mean free path of $l_{\mathrm{p}}=0.055 \mathrm{~mm}$, which is a reasonable scale considering the shortest of the sample dimensions $(0.04 \times 0.50 \times 1.92) \mathrm{mm}^{3}$. This nice agreement points towards a negligible electronic contribution to $\kappa$ at low $T$ and thus to a finite gap at every $\boldsymbol{k}$ point.

Figure 6.4 presents the thermal conductivity at zero field for temperatures up to $100 \mathrm{~K}$. At $T_{\mathrm{c}}$, a clear drop is visible followed by a hump at slightly lower $T$. In general, the thermal conductivity both in the normal state $\kappa^{\mathrm{n}}$ and in the superconducting state $\kappa^{\mathrm{s}}$ consists of a phonon and an electron contribution

$$
\begin{aligned}
\kappa^{\mathrm{n}} & =\kappa_{\mathrm{e}}^{\mathrm{n}}+\kappa_{\mathrm{p}}^{\mathrm{n}}, \\
\kappa^{\mathrm{s}} & =\kappa_{\mathrm{e}}^{\mathrm{s}}+\kappa_{\mathrm{p}}^{\mathrm{s}} .
\end{aligned}
$$

Assuming an $s$-wave superconductor, the drop can be attributed to the decreasing number of electronic heat carriers, hence, $\kappa_{\mathrm{e}}^{\mathrm{s}}$ decreases rapidly. The hump indicates an enhanced mean free path of phonons due to a decreasing number of electronic scattering centres. Therefore, $\kappa_{\mathrm{p}}^{\mathrm{s}}$ increases below $T_{\mathrm{c}}$. In the spirit of the theory of Bardeen, Rickayzen, and Tewordt (BRT) Bar59 (for an introduction see Section 2.6.1), we plotted a qualitative temperature dependence for both $\kappa_{\mathrm{p}}$ and $\kappa_{\mathrm{e}}$ in the inset of Figure 6.4 . 


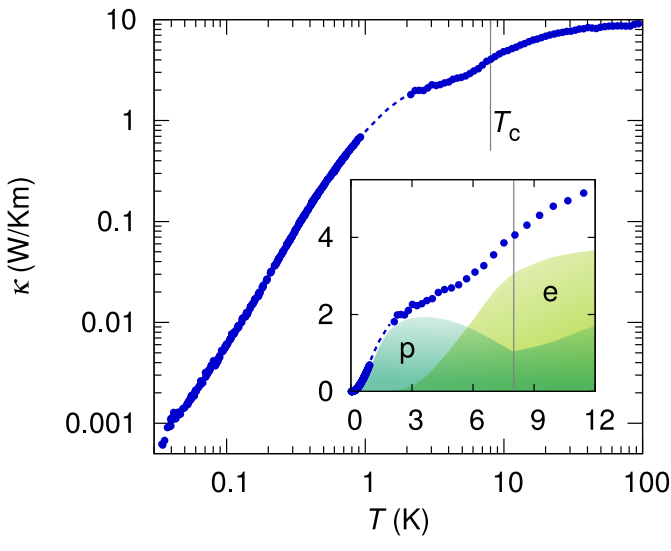

Figure 6.4: Temperature dependence of the thermal conductivity at $H=0$ up to $100 \mathrm{~K}$. Main panel: In the double-logarithmic representation, a clear but smooth drop of $\kappa(T)$ at $T_{\mathrm{c}}$ is visible. A hump appears at lower $T$. Both can be attributed to an decreasing number of electrons as heat carriers and as scattering centres for phonons, respectively. Inset: $\kappa(T)$ on a linear scale. For an $s$-wave superconductor, one expects a rapidly decreasing $\kappa_{\mathrm{e}}$ (e) below $T_{\mathrm{c}}$, while $\kappa_{\mathrm{p}}(\mathrm{p})$ increases forming a hump. Their $T$ dependence is inspired by the theory of BRT for $s$-wave superconductors. The dashed lines are guides to the eye.

\subsection{2 $H$ Dependence of the Thermal Conductivity}

\section{General Aspects}

In a type-II superconductor, a magnetic field is completely expelled up to $H_{\mathrm{c} 1}$. In $\mathrm{LaPt}_{4} \mathrm{Ge}_{12}$, $H_{\mathrm{c} 1}$ is very small with $14 \mathrm{mT}$ at $1.5 \mathrm{~K}$ [W. Schnelle, private communication]. Therefore, we do not consider this field region in detail here. Usually, the thermal conductivity stays almost constant below $H_{\mathrm{c} 1}$ as a function of field [Low70; Gup72; Boa03. However, there are drastic changes above $H_{\mathrm{c} 1}$ due to the properties of the vortex state.

A magnetic field $H>H_{\mathrm{c} 1}$ induces vortices along the field direction. The core of a vortex is in the normal state. Hence, in addition to the delocalized quasiparticles due to thermal excitations above the gap, there are quasiparticles from the core region, which can contribute to the heat flow. The low-energy fraction of those additional quasiparticles is localized within the vortex core, while the higher energy quasiparticles can extend further out Hes90. With increasing field the number of vortices increases, their separation decreases, and the core states can overlap with states from neighbouring vortices. The tunnelling between the cores contributes to a heat flow perpendicular to the magnetic field Gol11.

Around the vortex core, a supercurrent flows, the amplitude of which decays over a distance roughly equal to the penetration depth $\lambda$. The supercurrent with the velocity $\boldsymbol{v}_{\mathrm{S}}$ leads to a Doppler shift of the energy of delocalized quasiparticles $\epsilon(\boldsymbol{k}) \rightarrow \epsilon(\boldsymbol{k})-\hbar \boldsymbol{k} \cdot \boldsymbol{v}_{\mathrm{S}}(H)$ Cyr65 Vol93. This changes the excitation spectrum, increases the density of states (DOS), and effectively lowers the gap $\Delta$ for $\boldsymbol{k}$ directions with a component parallel to $\boldsymbol{v}_{\mathrm{S}}$.

These magnetic-field-induced changes in a type-II superconductor affect the thermal conductivity in different ways:

- Additional core states increase $\kappa_{\mathrm{e}}^{\mathrm{s}}$ Low70, Car65, Sch70, Gol11].

- The Doppler-shifted energy of delocalized quasiparticles increases $\kappa_{\mathrm{e}}^{\mathrm{s}}$ Vol93, Küb98 Vek99b.

- Scattering of electrons and phonons on the vortex lattice decreases $\kappa_{\mathrm{e}}^{\mathrm{s}}$ and $\kappa_{\mathrm{p}}^{\mathrm{s}}$ Low70 Car65; Küb98.

All of these effects are anisotropic with respect to the angle between current and field and also with respect to the angle between field and wave vector Bra67; Mak67; Küb98 

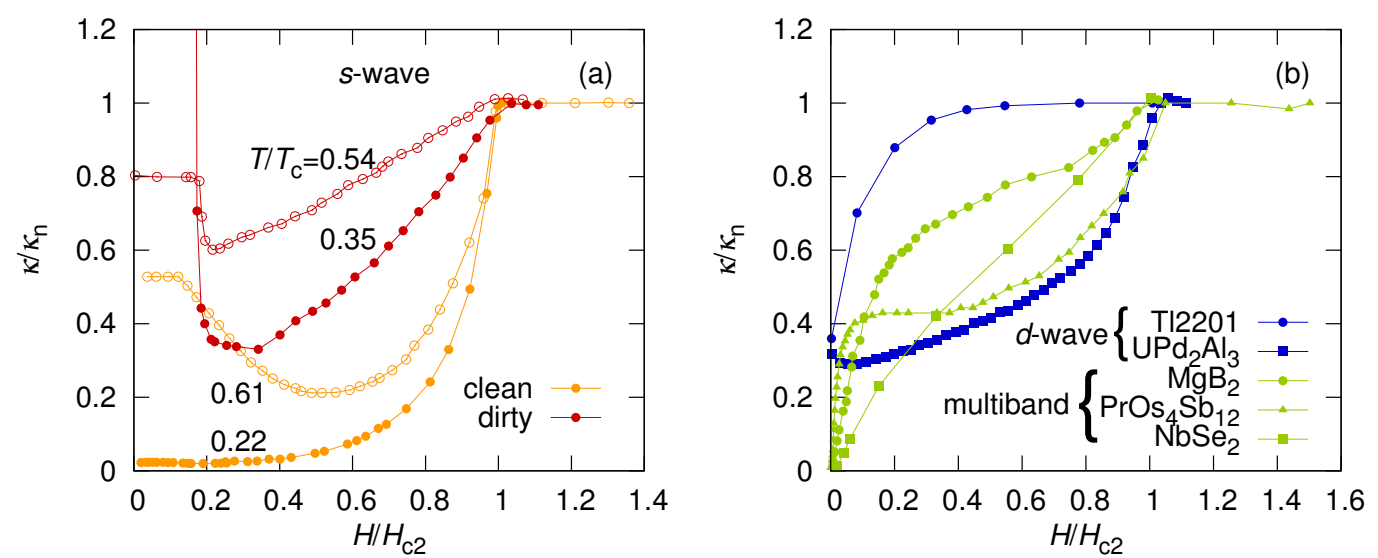

Figure 6.5: Field-dependent thermal conductivity of different compounds. (a) The thermal conductivity of a clean $s$-wave superconductor ( $\mathrm{Nb}$ with $T_{\mathrm{c}}=9.1 \mathrm{~K}$ Low70 $)$ shows a very large slope close to $H_{\mathrm{c} 2}$ compared to a dirty one $\left(\mathrm{Ta}_{80} \mathrm{Nb}_{20}\right.$ with $T_{\mathrm{c}}=4.6 \mathrm{~K}$ Low70] $)$. Both develop a minimum at intermediate fields for finite $T$. (b) Multiband superconductors typically show a change of curvature in $\kappa(H)$ as in the case of $\mathrm{MgB}_{2}$ (data shown for $T / T_{\mathrm{c}}=0.02, T_{\mathrm{c}}=38.1 \mathrm{~K}$ ) Sol02 or $\mathrm{NbSe}_{2}\left(T / T_{\mathrm{c}} \rightarrow 0, T_{\mathrm{c}}=7.0 \mathrm{~K}\right)$ Boa03. There are also hints that the skutterudite $\mathrm{PrOs}_{4} \mathrm{Sb}_{12}$ is a multiband superconductor $\left(T / T_{\mathrm{c}}=0.03, T_{\mathrm{c}}=1.75 \mathrm{~K}\right)$ Sey06b. The typical unconventional superconductors $\mathrm{Tl}_{2} \mathrm{Ba}_{2} \mathrm{CuO}_{6+\delta}\left(T / T_{\mathrm{c}} \rightarrow 0, T_{\mathrm{c}}=15 \mathrm{~K}\right)$ Pro02 and $\mathrm{UPd}_{2} \mathrm{Al}_{3}\left(T / T_{\mathrm{c}}=0.18, T_{\mathrm{c}}=2.0 \mathrm{~K}\right)$ Wat04 show a large residual term, and a steep slope at low fields (especially $\mathrm{Tl}_{2} \mathrm{Ba}_{2} \mathrm{CuO}_{6+\delta}$ ), which can be superimposed by a minimum at low fields and finite $T$ as observed in $\mathrm{UPd}_{2} \mathrm{Al}_{3}$.

Low70]. In the following we restrict the discussion to our case of a field perpendicular to the current. The thermal conductivity of selected materials with different pairing states, which are discussed below, is plotted in Figure 6.5.

For an $s$-wave superconductor at low temperatures and $H<H_{\mathrm{c} 1}$, the main contribution to the thermal transport is due to phonons. Slightly increasing the field $H_{\mathrm{c} 1}<H \ll H_{\mathrm{c} 2}$ lowers their mean free path due to scattering off the vortex lattice. At the same time, there is no significant change in the electronic contribution. At high fields $H \lesssim H_{\mathrm{c} 2}$, the contribution of the core states increases rapidly. Both together, the development of the phonon and the electron contribution, gives rise to a minimum in $\kappa(H)$ at intermediate fields.

For $s$-wave superconductors at higher temperatures, there is a considerable contribution of delocalized quasiparticles at $H<H_{\mathrm{c} 1}$. Increasing the field slightly $H_{\mathrm{c} 1}<H \ll H_{\mathrm{c} 2}$ also decreases their mean free path due to scattering on vortices, similar to phonons. At higher fields, the DOS of the delocalized quasiparticles increases due to the Doppler shift as well as the contribution from localized core states. Again, we observe a minimum in $\kappa^{\mathrm{s}}(H)$ at intermediate fields.

The minimum is temperature dependent. It does not exist in the limit $T \rightarrow 0$ but becomes more and more pronounced with increasing temperature. At very low $T$, it is mainly due to the reduction of the phonon mean free path, while at higher temperatures it is due to the electronic mean free path in the case of clean superconductors and still due to the phonon mean free path in the case of dirty superconductors [Pes74].

There are some attempts to calculate the field-dependent thermal conductivity for an $s$ - 


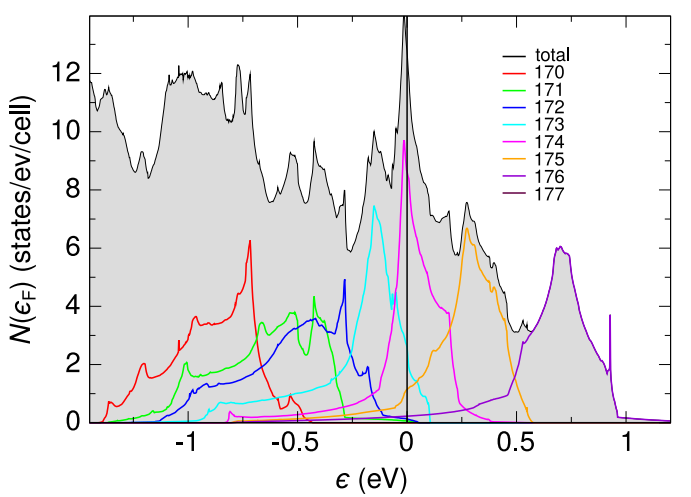

Figure 6.6: Calculated DOS of $\mathrm{LaPt}_{4} \mathrm{Ge}_{12}$. There are three bands contributing most to $N\left(\epsilon_{\mathrm{F}}\right)$ at the Fermi energy: 173, 174, and 175. Calculations by H. Rosner. For details of the calculations see also Gum08.

wave superconductor. Dubek et al. Dub64, Dub63 treated the field dependence with the help of a spatially averaged gap $\overline{\Delta(T, H)}$ inserted into the theoretical description of $\kappa(T)$ by Bardeen, Rickayzen, and Tewordt Bar59]. For fields close to $H_{\mathrm{c} 2}$, Maki [Mak67 calculated $\kappa_{\mathrm{e}}^{\mathrm{s}}(H)$ in a clean superconductor and obtained $\kappa_{\mathrm{e}}^{\mathrm{s}}(H) \propto\left(H_{\mathrm{c} 2}-H\right)^{1 / 2}$. For the case of a dirty superconductor, Caroli et al. Car65] obtained $\kappa_{\mathrm{e}}^{\mathrm{s}}(H) \propto\left(H_{\mathrm{c} 2}-H\right)$ close to $H_{\mathrm{c} 2}$. Both cases could nicely be observed in Nb alloys of different quality Low70]. These experiments also confirm the above qualitative picture (see Figure 6.5(a)).

There are superconductors which involve more than one band. The textbook example for this case is $\mathrm{MgB}_{2}$. Multiband superconductors can show complex behaviour in magnetic fields in the case of different sizes $\Delta_{i}$ of the gaps. At finite fields, the tunnelling probability for the core states is controlled by the ratio of the coherence length $\xi$ and the vortex distance Gol11, the latter of which decreases with increasing field. A small energy gap on one of the Fermi-surface sheets leads to an increase of $\xi$ and thus an increase of $\kappa$ at low fields Kus02 Gol11]. This behaviour was observed in $\mathrm{MgB}_{2}$ Sol02] or $\mathrm{NbSe}_{2}$ Boa03 (see Figure 6.5(b)).

Let us now consider the case of unconventional superconductors. The main difference of superconductors with nodes in the gap compared to fully gapped superconductors is the larger number of delocalized quasiparticles around the nodes, which can be excited even at low temperatures. Hence, the transport at low $T$ is not dominated by phonons but by those quasiparticles along the nodal direction. Applying a magnetic field $H_{\mathrm{c} 1}<H \ll H_{\mathrm{c} 2}$, their DOS grows rapidly due to the Doppler shift. Hence, we expect a larger slope of $\kappa^{\mathrm{s}}(H)$ compared to the $s$-wave case. This can be observed, e.g., in the typical cuprate d-wave superconductors, such as $\mathrm{Tl}_{2} \mathrm{Ba}_{2} \mathrm{CuO}_{6+\delta}$ Pro02]. The slope can vary depending on the type of nodes and the purity of the sample Küb98, Vek99b, Won01] (see e.g. $\mathrm{UPd}_{2} \mathrm{Al}_{3}$ Wat04 in Figure 6.5(b)). At higher temperatures, this effect is again superimposed by electron-vortex scattering at low fields Vek99b Küb98 leading to a minimum in $\kappa^{\mathrm{s}}(H)$ similar to the $s$-wave superconductors described above. This behaviour could be observed experimentally e.g. in $\mathrm{UPd}_{2} \mathrm{Al}_{3}$ [Wat04] and $\mathrm{Sr}_{2} \mathrm{RuO}_{4}$ Iza01] (see Figure 6.5(b)).

\section{Clean or Dirty Limit}

The above general considerations illustrate that the properties of superconductors exhibit distinct differences between the cases of clean and dirty materials. Generally, a superconductor is in the clean limit if the coherence length $\xi$ is much smaller than the mean free path $l_{\mathrm{e}} \gg \xi$. The dirty limit is characterized by $l_{\mathrm{e}} \leq \xi$. 
The Ginzburg-Landau coherence length $\xi$ can be calculated by Poo00]

$$
H_{\mathrm{c} 2}=\frac{\Phi_{0}}{2 \pi \xi^{2}}=\frac{h}{4 e \pi \xi^{2}}
$$

to $\xi=1.53 \cdot 10^{-8} \mathrm{~m}$ in $\mathrm{LaPt}_{4} \mathrm{Ge}_{12}$.

The mean free path is often estimated from the relation $\kappa_{\mathrm{e}}=C_{\mathrm{e}} v_{\mathrm{F}} l_{\mathrm{e}} / 3$ for the electronic thermal conductivity. Using $C_{\mathrm{e}}=\gamma T, \kappa_{\mathrm{e}}=\kappa_{\mathrm{WF}}=L_{0} T / \rho, L_{0}=3 k_{\mathrm{B}}^{2} / 2 e^{2}$, and $\gamma=$ $\pi^{2} k_{\mathrm{B}}^{2} N\left(\epsilon_{\mathrm{F}}\right) / 3$, we find three possibilities to estimate $l_{\mathrm{e}}$

$$
l_{\mathrm{e}}=\frac{3 \kappa(T \rightarrow 0)}{v_{\mathrm{F}} C_{\mathrm{e}}(T \rightarrow 0)}, \quad l_{\mathrm{e}}=\frac{3 L_{0}}{\rho_{0} \gamma v_{\mathrm{F}}}, \quad l_{\mathrm{e}}=\frac{27}{2 \pi^{2}} \frac{1}{e^{2} N\left(\epsilon_{\mathrm{F}}\right) v_{\mathrm{F}} \rho_{0}},
$$

all of which require the knowledge of the Fermi velocity $v_{\mathrm{F}}$ and additionally the knowledge of the density of states $N\left(\epsilon_{\mathrm{F}}\right)$ in the last case. We extract these values from band-structure calculations by H. Rosner ( $c f$. Figure 6.6). They revealed three bands contributing most to $N\left(\epsilon_{\mathrm{F}}\right)$. These bands have the following parameters

$$
\begin{aligned}
& \text { band 173: } N\left(\epsilon_{\mathrm{F}}\right) \approx 2 \frac{\text { states }}{\text { eVcell }}, \quad v_{\mathrm{F}}=0.02 . .1 .23 \cdot 10^{6} \frac{\mathrm{m}}{\mathrm{s}}, \\
& \text { band 174: } N\left(\epsilon_{\mathrm{F}}\right) \approx 8 \frac{\text { states }}{\text { eVcell }}, \quad v_{\mathrm{F}}=0.05 . .0 .97 \cdot 10^{6} \frac{\mathrm{m}}{\mathrm{s}}, \\
& \text { band 175: } N\left(\epsilon_{\mathrm{F}}\right) \approx 1 \frac{\text { states }}{\text { eVcell }}, \quad v_{\mathrm{F}}=0.12 . .1 .12 \cdot 10^{6} \frac{\mathrm{m}}{\mathrm{s}} .
\end{aligned}
$$

The unit-cell volume is $V_{\text {cell }}=641.28 \AA$ Gum08. The above values lead to a maximum, minimum and average mean free path for the three alternative estimations

$$
\begin{aligned}
& l_{\max }=4 \cdot 10^{-7} \mathrm{~m}, \quad 3 \cdot 10^{-7} \mathrm{~m}, \quad 4 \cdot 10^{-6} \mathrm{~m}, \\
& l_{\min }=6 \cdot 10^{-9} \mathrm{~m}, \quad 5 \cdot 10^{-9} \mathrm{~m}, \quad 2 \cdot 10^{-8} \mathrm{~m} \text {, } \\
& l_{\text {avg }}=2 \cdot 10^{-8} \mathrm{~m}, \quad 1 \cdot 10^{-8} \mathrm{~m}, \quad 6 \cdot 10^{-8} \mathrm{~m} \text {. }
\end{aligned}
$$

All three methods give an average mean free path which is roughly the coherence length $l_{\mathrm{e}} \approx \xi$. Therefore, our sample is located in the dirty limit.

\section{Experimental Results}

We now turn to the field-dependent $\kappa(H) / T$ for $\mathrm{LaPt}_{4} \mathrm{Ge}_{12}$, which is shown in Figure 6.7(a) for different temperatures between $290 \mathrm{mK}$ and $650 \mathrm{mK}$ together with a zero-temperature extrapolation. The measurements were performed on increasing field and are consistent with the temperature dependence shown in Figure 6.3. Comparison with measurements during decreasing field showed no hysteresis. A clear kink is visible at $H_{\mathrm{c} 2}=1.4 \mathrm{~T}$, which is slightly lower than $1.6 \mathrm{~T}$ from the extrapolation of the specific-heat data obtained at $T \geq 1.8 \mathrm{~K}$ on polycrystals Gum08. This $H_{\mathrm{c} 2}$ is also much smaller than the $2.5 \mathrm{~T}$ obtained from our resistivity measurements ( $c f$. Section 6.3). The latter value, however, is most likely higher due to surface or impurity effects. The good agreement of $H_{\mathrm{c} 2}$ determined from thermal conductivity and from specific heat proves the bulk sensitivity of the thermal-conductivity measurement. Below $H_{\mathrm{c} 2}$, the thermal conductivity shows an almost linear field dependence 

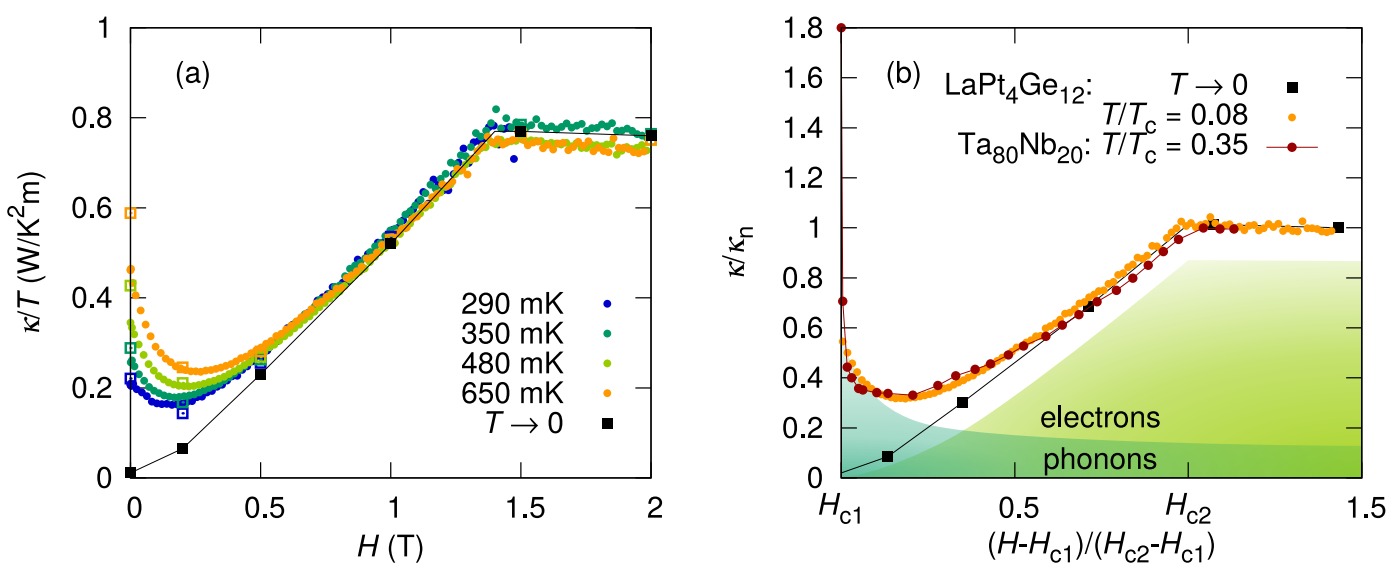

Figure 6.7: Magnetic-field dependence of the thermal conductivity. Dots represent field sweeps after zero-field-cooling. Open squares are extracted from temperature sweeps after field-cooling, filled squares are their zero-temperature extrapolations. The line is a guide to the eye. (a) $\kappa / T(H)$ is almost linear and develops a minimum at low fields which becomes more pronounced with higher temperature. (b): We compare the data from (a) for $650 \mathrm{mK}$ and $T \rightarrow 0$ with the dirty $s$-wave superconductor $\mathrm{Ta}_{80} \mathrm{Nb}_{20}$ Low70]. Note the different scaling of the field axis to account for the different $H_{\mathrm{c} 1}$ and $H_{\mathrm{c} 2}$. The shaded areas illustrate qualitatively how the electronic and the phonon contribution change with field Dub63. Pes74.

in the zero-temperature limit. For finite temperatures, a minimum appears at low fields, which shifts to higher fields and becomes more pronounced as the temperature is increased.

The specific heat and the temperature dependence of the thermal conductivity point towards a single BCS $s$-wave gap. Additionally, an estimation of the mean free path and the coherence length suggests that our sample is in the dirty limit. Therefore, we will first compare our experimental results on the field-dependent thermal conductivity with predictions and other examples of dirty $s$-wave superconductors, which were described in detail above. The predictions of a linear slope of $\kappa(H)$ close to $H_{\mathrm{c} 2}$ and a minimum at intermediate fields, which is absent for $T \rightarrow 0$ but increases in amplitude with increasing $T$, fit nicely to our observations. In Figure 6.7(b), we directly compare our result with measurements on the dirty $s$-wave superconductor $\mathrm{Ta}_{80} \mathrm{Nb}_{20}$ Low70. The results for other examples of this class look similar Gup72; Low70; Mut68; Dub64]. Although the lowesttemperature data available for $\mathrm{Ta}_{80} \mathrm{Nb}_{20}$ are still taken at higher $T$ both absolute $(T=$ $1.61 \mathrm{~K})$ and relative $\left(T / T_{\mathrm{c}}=0.35\right)$ than our measurements, they fit almost perfectly onto our results for $T=650 \mathrm{mK}$ or $T / T_{\mathrm{c}}=0.08$, respectively. The main difference is the very large thermal conductivity at $H=H_{\mathrm{c} 1}$ for $\mathrm{Ta}_{80} \mathrm{Nb}_{20}$, which is due to the high phonon thermal conductivity $\kappa_{\mathrm{p}}^{\mathrm{s}}$ at higher temperature. For $\mathrm{LaPt}_{4} \mathrm{Ge}_{12}$, we expect the phonon contribution to increase considerably with increasing $T$ as can be seen in Figure 6.4 This suggests an increase at $H=H_{\mathrm{c} 1}$ for higher $T$, similar to $\mathrm{Ta}_{80} \mathrm{Nb}_{20}$, also in $\mathrm{LaPt}_{4} \mathrm{Ge}_{12}$.

The very good agreement with results for a dirty $s$-wave superconductor imply that the field dependence of the thermal conductivity is composed of a phonon part $\kappa_{\mathrm{p}}^{\mathrm{s}}$, which decreases with increasing field due to scattering on vortices, and an electronic contribution, which increases due to an enhanced number of localized states in the vortex cores tunnelling 
to ever closer neighbouring vortices.

Since other skutterudites are discussed in terms of multiband superconductivity Sey06b; Zha13, we also want to study this possibility for $\mathrm{LaPt}_{4} \mathrm{Ge}_{12}$. As shown in Figure 6.5(b) for $\mathrm{MgB}_{2}$ and $\mathrm{PrOs}_{4} \mathrm{Sb}_{12}$, a typical feature of $\kappa(H)$ is a plateau at intermediate fields, which is attributed to the suppression of the smaller of the two gaps. We do not observe such change of curvature in $\mathrm{LaPt}_{4} \mathrm{Ge}_{12}$. However, as in the case of $\mathrm{NbSe}_{2}$ (Figure 6.5(b)), this change can be very weak. Additionally, we expect from specific heat either two very similar gaps or only a very small contribution of a second gap. In the first case, the contribution to $\kappa(H)$ can be very similar. This case is generally difficult to distinguish from a single-band superconductor with any method. For the second case, one would expect a small field scale involved, which could entirely be covered by the phonon contribution at finite temperatures and missed in the zero-temperature limit due to the small number of points at low $H$.

Let us now compare our results with observations and predictions for the case of nodes in the superconducting gap. There are both theoretical Vek99b Küb98 and experimental Mac12; Iza01, Wat04 reports on a minimum in $\kappa(H)$ for unconventional superconductors. The slope above the minimum can vary considerably depending on the type of gap and the purity of the sample (see Figure 6.5(b)). Hence, these properties do not rule out nodes in the gap of $\mathrm{LaPt}_{4} \mathrm{Ge}_{12}$ completely. However, one expects a sizeable residual term for $T \rightarrow 0$ in a nodal superconductor. In contrast, both single-band (Figure 6.5(a)) and multiband (Figure 6.5(b)) $s$-wave superconductors show a tiny residual term exactly as our results on $\mathrm{LaPt}_{4} \mathrm{Ge}_{12}$.

\subsubsection{Field-angle Dependence of the Thermal Conductivity}

The variation of the thermal conductivity with the magnetic field direction is a powerful tool not only to probe the existence of nodes in the superconducting gap, but also to gain information about their position in $\boldsymbol{k}$ space Vek99a; Yu95, Mat06]. The Doppler-shifted energy of the delocalized quasiparticles around the nodes leads to a field-angle-dependent DOS and thus to an angle-dependent thermal conductivity $\kappa(\theta, \phi)$ due to the term $\hbar \boldsymbol{k} \cdot \boldsymbol{v}_{\mathrm{S}}(H)$. The angles $\theta$ and $\phi$ denote, respectively, the azimuthal and polar angle in spherical coordinates. The oscillation patterns in $\kappa(\theta, \phi)$ depend on the specific gap structure.

However, the analysis of these experiments remains a challenging task. First, oscillations in $\kappa(\theta, \phi)$ can have several other reasons, some of which are hard to distinguish from gap nodes. If the oscillations proved to be due to nodes, it is secondly very difficult to extract their $\boldsymbol{k}$ position, because the magnetic field direction not only determines the DOS but also the scattering time of the quasiparticles. Additionally, sign changes of the oscillations are predicted as a function of magnetic field strength and temperature [Vor06, Das12]. For this reason, we focus on the question whether the origin of the oscillation patterns lies within a nodal structure of the superconducting gap.

Figure 6.8 shows the field-angle dependence of $\kappa(\phi)$ at $500 \mathrm{mK}=0.06 T_{c}$ and for different magnetic fields between $H=0$ and $2 \mathrm{~T}$. We restrict our experiments to a rotation of $\boldsymbol{H}$ within the plane perpendicular to $\boldsymbol{j}$ ( $c f$. Figure 6.9). Our experiments do not show any dependence on zero-field versus field-cooling condition, and field cooling at different angles gives consistent results. At zero field, $\kappa(\phi)=$ const., which is expected for the magnet rotating around the sample with $H=0$. Our data at finite field show nicely resolved oscillation patterns, which vary drastically by changing the magnetic field. The oscillations below $H_{\mathrm{c} 2}$ are slightly off-centred, which indicates that our sample was misaligned by $\phi_{0}=7^{\circ}$ 


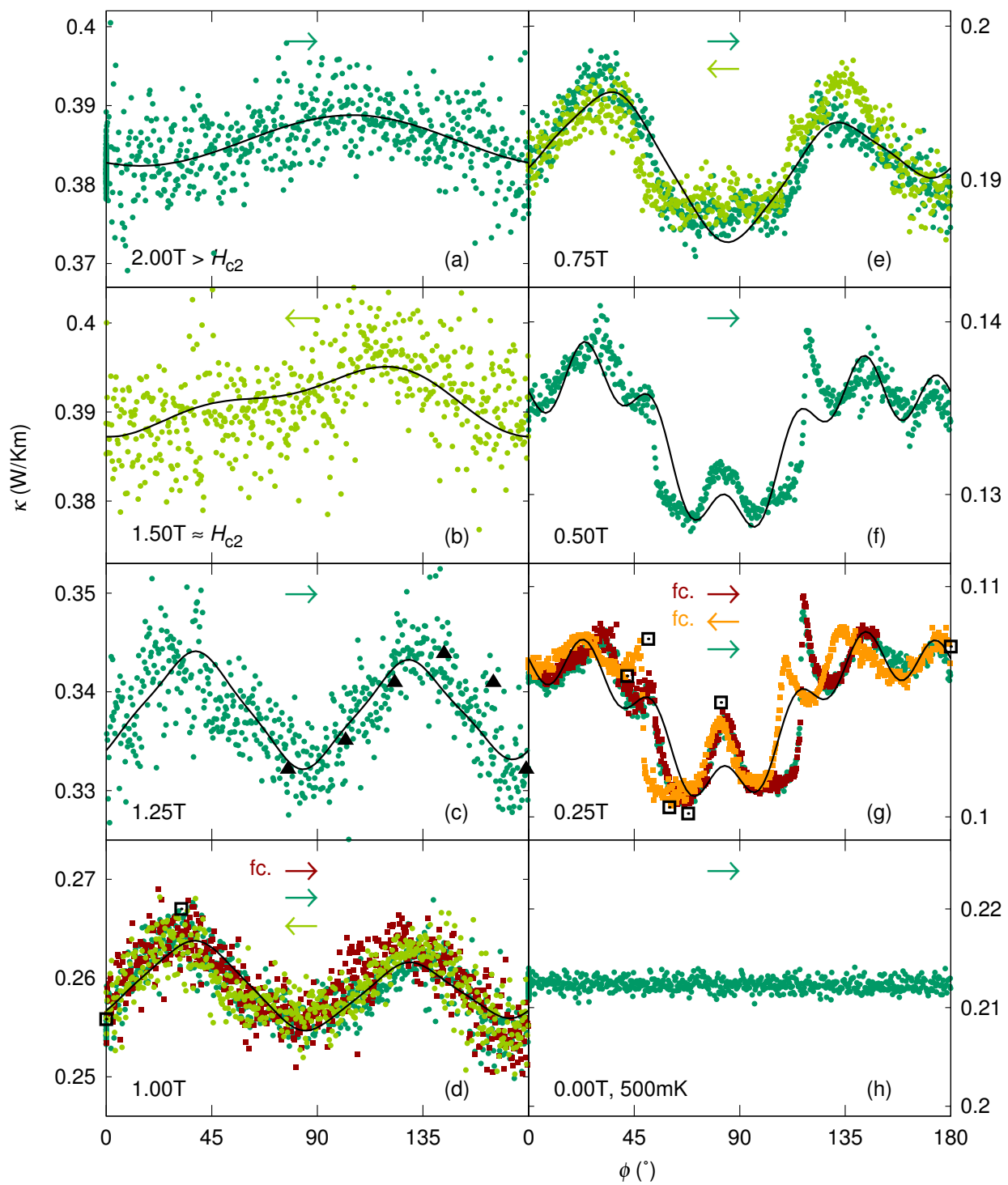

Figure 6.8: Field-angle dependence of the thermal conductivity at $T=500 \mathrm{mK}$. Data were taken in zero-field cooling (dots) at $0^{\circ}$ or $180^{\circ}$ depending on the sweep direction indicated by the arrows. $(\mathrm{d}),(\mathrm{g})$ : The results are consistent with sweeps under field-cooling (filled squares) again at $0^{\circ}$ or $180^{\circ}$, and with single data points extracted from $T$ sweeps under field cooling at different angles (open squares). Lines represent fits to the data according to Equation 6.14 In (d),(e),(g) this fit is performed to the data for sweeps along $0^{\circ} \rightarrow 180^{\circ}$. (c) The $H_{\mathrm{c} 2}(\phi)$ anisotropy determined from torque measurements För (triangles) can nicely account for the 4-fold oscillation. 

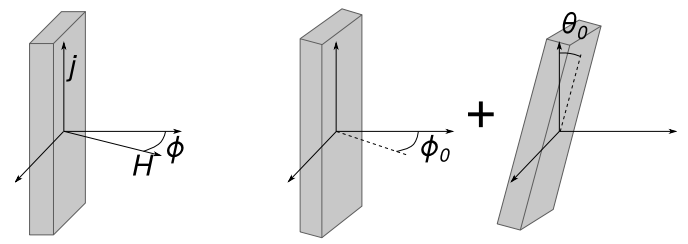

Figure 6.9: Orientation of sample, current $\boldsymbol{j}$, and magnetic field $\boldsymbol{H}$. Left: The field rotates around the sample varying the angle $\phi$. Middle: The field-angle-dependent measurements revealed a misalignment of $\phi_{0}$ in direction of the rotation. Right: Furthermore, the measurement results for a rotation with a field of $H=2.0 \mathrm{~T}$ can be explained by an additional tilting of the sample along $\theta_{0}$.

(cf. Figure 6.9). Only at low fields (see Figure 6.8(g)), we find hysteresis effects together with a quite sharp anomaly around $55^{\circ}$ and $110^{\circ}$. They are probably due to vortex pinning and associated jumps in the vortex lattice during rotation.

In the following, we will decompose the patterns in Figure 6.8 and model every oscillation with a sine function with an amplitude $A_{x}$ and a phase shift $\phi_{x}(x=1 . .4)$. The complete model function is

$$
\begin{aligned}
\kappa(\phi)=\kappa_{0}+A_{1} \sin \left(2\left(\phi+\phi_{1}\right)\right)+A_{2} \sin ( & \left.4\left(\phi+\phi_{2}\right)\right) \\
& +A_{3} \sin \left(2\left(\phi+\phi_{3}\right)\right)+A_{4} \sin \left(12\left(\phi+\phi_{4}\right)\right) .
\end{aligned}
$$

The field-independent shifts $\phi_{x}$ are fully determined by the misalignment $\phi_{0}$ of the sample as is described below in detail. A fit of Equation 6.14 for each data set in Figure 6.8 with $\kappa_{0}$ and $A_{x}$ as free parameters leads to the lines shown in every sub-plot. We set $A_{3}=0$ for $1.5 \mathrm{~T}$ and $2.0 \mathrm{~T}$ due to the large noise level. We plot the field dependence of the amplitudes $A_{x}$ in Figure 6.10 As an overview, the origins of all four oscillations are listed in Table 6.1.

The 2-fold oscillation at $2 \mathrm{~T}$ above $H_{\mathrm{c} 2}$ is not symmetric to $\left(90^{\circ}-\phi_{0}\right)$. It can nicely be explained by a slightly tilted sample away from $\boldsymbol{H} \perp \boldsymbol{j}$ assuming a difference of the thermal conductivity parallel and perpendicular to the field $\kappa_{\perp \boldsymbol{H}} \neq \kappa_{\| \boldsymbol{H}}$. Experimentally, a tilting by an angle $\theta_{0}$ is most likely to occur around the axis $\boldsymbol{H}\left(\phi=90^{\circ}\right)$ as indicated in Figure 6.9. The shift $\phi_{1}=59^{\circ}$ of the resulting sine function is then solely determined by $\phi_{0}$. Its amplitude $A_{1}$ depends on the ratio $\kappa_{\perp \boldsymbol{H}} / \kappa_{\| \boldsymbol{H}}$. We describe this oscillation with the second term in Equation 6.14. Its amplitude $A_{1}$ changes sign twice at $H_{\mathrm{c} 2}$ and at very low fields. Sign changes of $A_{1}$, i.e., changes in the anisotropy from $\kappa_{\perp \boldsymbol{H}}<\kappa_{\| \boldsymbol{H}}$ to $\kappa_{\perp \boldsymbol{H}}>\kappa_{\| \boldsymbol{H}}$ are expected [Mak67] and observed [Low70 in the mixed state.

Below $H_{\mathrm{c} 2}$, a 4 -fold oscillation appears with a phase shift $\phi_{2}=16^{\circ}$ (third term in Equation 6.14). Three different origins are possible: an anisotropy of $H_{\mathrm{c} 2}$, Fermi-surface or Fermivelocity anisotropies, or nodes/extrema in the gap. Torque measurements revealed a 4 -fold $H_{\mathrm{c} 2}(\phi)$ anisotropy [För, which can be converted into a $\kappa(\phi)$ anisotropy using the results of $\kappa(H)$ close to $H_{\mathrm{c} 2}$. We include the so obtained values into Figure 6.8(c). They fit almost perfectly onto our measured $\kappa(\phi)$. Hence, the 4-fold oscillation can be entirely ascribed to a $H_{\mathrm{c} 2}$ anisotropy. The fit values for $A_{2}$, which reach a maximum right at $H_{\mathrm{c} 2}$ and decrease monotonically towards $H=0$, corroborate this result.

Below $1 \mathrm{~T}$, we observe hysteretic jumps in $\kappa(\phi)$ at around $55^{\circ}$ and $110^{\circ}$ due to the pinning of the moving vortex lattice. Hysteresis effects due to pinning were also observed before Aub97 in other compounds. In the absence of any clear model for these hysteresis effects, we include them into a two-fold sine function (fourth term in Equation 6.14), which we use to model the underlying two-fold oscillation. This oscillation and the jumps appear again 


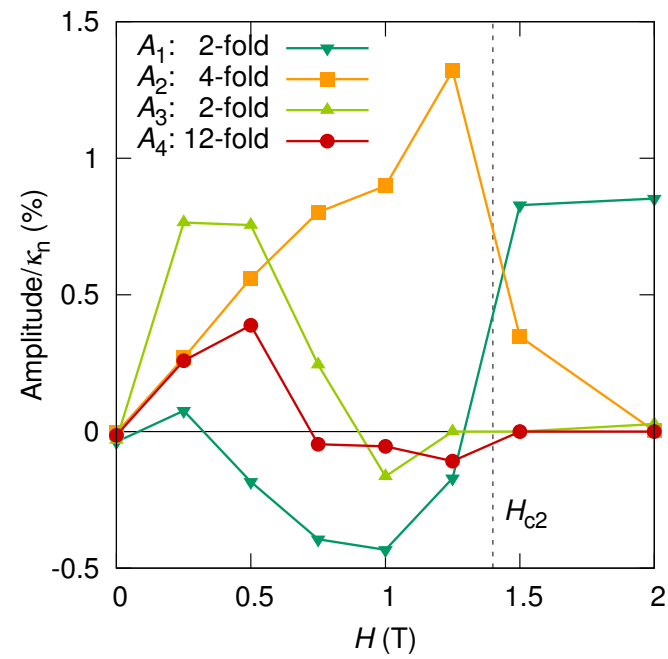

Figure 6.10: Amplitudes $A_{x}$ for the oscillations found in the field-angle dependence of $\kappa(\phi)$. Amplitudes are determined by a fit of Equation 6.14 (lines in Figure 6.8 to $\kappa(\phi)$ (data in Figure 6.8) and normalized by $\kappa_{n}=$ $\kappa\left(H_{\mathrm{c} 2}, T=500 \mathrm{mK}\right)$.

symmetric to $\left(90^{\circ}-\phi_{0}\right)$, hence $\phi_{3}=16^{\circ}$. The amplitude $A_{3}$ reaches its maximum at low fields between $0.2 \mathrm{~T}$ and $0.5 \mathrm{~T}$. The field dependence of $\kappa$ in Figure 6.7 indicates that the main contribution in this field range comes from phonons. Therefore, it is natural to ascribe the 2 -fold anisotropy to an anisotropic mean free path of the phonons. We can simultaneously account for demagnetization effects, since they also lead to two-fold oscillations.

Below $0.75 \mathrm{~T}$, a 12 -fold oscillation appears with the same phase shift $\phi_{4}=16^{\circ}$ (fifth term in Equation 6.14). In general, this feature can have several reasons. First, the Fermi surface or Fermi velocity in this compound induces higher order oscillations than the 4-fold cubic symmetry. Such unusual oscillation patterns resulting from Fermi-surface anisotropies are indeed predicted Das12]. Second, the 12-fold oscillation stems from nodes or deep minima in the gap. Since we do not observe any signature of nodes in the temperature-dependent thermal conductivity, these extrema could only be shallow. They may be connected to a possible second gap with a very small contribution, since the oscillation disappears at higher fields. Third, the motion of the vortices leads to anisotropic scattering of phonons or electrons. The main contribution to $\kappa(H)$ at low fields is due to phonons. Similar to the contribution of the phonons to $\kappa(H)$, the 12-fold oscillation also flattens out for higher

\begin{tabular}{cccc}
\hline & Period & State & Origin of oscillation \\
\hline$A_{1}$ & 2 -fold & N,SC & Tilted sample $\left(\kappa_{\perp \boldsymbol{H}} \neq \kappa_{\| \boldsymbol{H}}\right)$ \\
$A_{2}$ & 4 -fold & SC & $H_{\mathrm{c} 2}$ anisotropy \\
$A_{3}$ & 2 -fold & SC & Demagnetization effects + phonon-vortex scattering + pinning \\
$A_{4}$ & 12 -fold & SC & Fermi surface/velocity anisotropy \\
& & & or gap nodes \\
& & or anisotropic phonon mean free path \\
\hline
\end{tabular}

Table 6.1: List of origins for observed oscillations in $\kappa(\phi)$. N: normal state, SC: superconducting state. We can unambiguously connect the first three oscillations to the listed origins. The source of the last one with a 12 -fold symmetry remains unresolved, but can be limited to three possibilities. 
fields. Therefore, a connection to the phonon scattering time seems likely, however, there is no obvious explanation for such a high frequency of oscillation.

\subsection{Conclusion}

We performed specific-heat and thermal-conductivity measurements to study the superconducting order parameter of $\mathrm{LaPt}_{4} \mathrm{Ge}_{12}$. Our specific-heat results can be described with a single BCS gap of the size $\Delta=2.0 k_{\mathrm{B}} T_{\mathrm{c}}$. If there is a second gap involved as in $\operatorname{PrPt}_{4} \mathrm{Ge}_{12}$, it has a very similar gap size or its contribution to the specific heat must either be very small or below our temperature limit.

The focus of our study was on the low-temperature thermal-transport studies and we used the specific-heat results to analyse them in detail. Both the temperature- and the magneticfield-dependent thermal conductivity are compatible with a BCS $s$-wave superconductor. No indications were found for a second gap. As for the specific heat, we can, however, not completely rule out either a very small second gap with a tiny contribution or two gaps with very similar gap sizes.

As a more complex probe of the order parameter, we also performed field-angle-dependent thermal-conductivity measurements. Previously, oscillation patterns in the angle dependence were used to determine the existence and the exact position of nodes in the gap of several unconventional superconductors. We were able to improve our set-up, to nicely resolve field-angle-dependent oscillations also in $\mathrm{LaPt}_{4} \mathrm{Ge}_{12}$. They consist of various components involving a lower, a higher, or the same symmetry compared to the underlying cubic crystal lattice. They could all be assigned to sources other than nodes in the gap, except of a fast 12 -fold pattern, whose origin stays unclear. All our results point towards conventional superconductivity. The rich pattern, that we found in the field-angle dependence, therefore, highlights the importance of a careful analysis of these kind of measurements. 


\section{Summary and Outlook}

In this dissertation, we set out to investigate phenomena in strongly correlated rare-earth intermetallic compounds, which are unconventional in the sense that they go beyond the fundamental concepts of condensed-matter physics - the Fermi-liquid theory and Landau's concept of symmetry-breaking phase transitions. To gain a deeper understanding of the underlying physical mechanisms of the unconventional behaviour, we tested the validity of established physical principles and laws using transport, particularly thermal-transport measurements at very low temperatures.

For our thermal-transport measurements, we used the standard one-heater-two-thermometers method to study the behaviour of our materials as a function of temperature in the steady-state procedure. In addition, we also implemented a pseudo-ac method to perform isothermal investigations as a function of an external parameter, in our case magnetic field and magnetic-field angle. The magnetic field introduces an additional energy scale into our systems. It tunes competing interactions, which are responsible for the interesting phenomena we want to investigate. Using our experimental set-ups we studied three specific scientific questions.

We first investigated the quantum critical point (QCP) in $\mathrm{YbRh}_{2} \mathrm{Si}_{2}$ - a point where a material undergoes a second-order phase transition at zero temperature driven not by thermal but by quantum fluctuations. The standard Hertz-Millis-Moriya theory is able to describe many of the observed QCPs, but $\mathrm{YbRh}_{2} \mathrm{Si}_{2}$, in which a tiny magnetic field induces such a transition, shows unconventional behaviour. There is an ongoing debate and there are several theoretical attempts to describe the $\mathrm{QCP}$ in $\mathrm{YbRh}_{2} \mathrm{Si}_{2}$. Our aim was to identify if a theory can be formulated in the framework of standard quasiparticles or if this picture breaks down at the QCP. An established test of the existence of quasiparticles is the WiedemannFranz law. It is a fundamental law, which relates the thermal and the electrical conductivity and which is observed in virtually all metals. It is valid in the zero-temperature limit if quasiparticles exist.

While we observe a validity on either side of the QCP, our extrapolation at the critical magnetic field suggests a violation of the Wiedemann-Franz law at the QCP. This implies a breakdown of the standard quasiparticle picture and puts strong constraints on every theory which aims to describe the $\mathrm{QCP}$ in $\mathrm{YbRh}_{2} \mathrm{Si}_{2}$. Three other groups repeated our measurements with essentially the same experimental results. However, their interpretations lead to a verification of the Wiedemann-Franz law. The different viewpoints are caused by an additional contribution in the heat transport visible both in zero field and at the critical field. We ascribe this contribution to transport by (para-)magnons. Its existence at the critical field was clearly confirmed only in the most recent study performed at the lowest temperatures reached so far. Unfortunately, it masks the low-temperature electronic thermal conductivity. Only a detailed investigation and a deeper understanding of this contribution 
can settle the ongoing debate about the Wiedemann-Franz law in $\mathrm{YbRh}_{2} \mathrm{Si}_{2}$.

In the second project, we investigated the evolution of the Kondo effect in magnetic fields with an energy of the order of the Kondo temperature. We studied the compounds $\mathrm{CeRu}_{2} \mathrm{Si}_{2}$ and $\mathrm{YbRh}_{2} \mathrm{Si}_{2}$, for which transitions at $8 \mathrm{~T}$ and $10 \mathrm{~T}$, respectively, were reported. The origins of these transitions are debated. While the experimental signatures in both compounds are quite different, similar interpretations can be found in literature. Two models are proposed: an abrupt breakdown of the Kondo effect or a topological change of the Fermi surface called Lifshitz transition. We used magnetic-field-dependent resistivity and thermopower measurements to study the field development of the quasiparticle bands in both materials in general and the reported transitions in particular. These probes are especially suitable due to their sensitivity to both changes in the density of states and in the scattering rate. The latter in particular renders transport measurements an ideal tool to detect Lifshitz transitions.

For $\mathrm{CeRu}_{2} \mathrm{Si}_{2}$, we demonstrated that the thermopower is consistent with a Lifshitz transition predicted by previous electrical-transport studies. However, rigid-band models seem insufficient to incorporate also thermodynamic observations and a satisfying global description is still missing. For $\mathrm{YbRh}_{2} \mathrm{Si}_{2}$, our measurements revealed in total three transitions at high fields instead of a single one at $10 \mathrm{~T}$. We found an unexpected validity of the Mott formula above $2 \mathrm{~T}$ and an extraordinary good agreement of field-dependent renormalized-band structure calculations with our and previous experimental results. These findings were used to identify Lifshitz transitions as the origin of all three transitions. They are superposed on a smooth suppression of the Kondo effect in magnetic field. The excellent agreement of experiment and theory proves that renormalized band calculations are a very suitable approach to describe Kondo lattices in magnetic field. The knowledge of the field development of the quasiparticle bands has a strong impact, e.g., on the interpretation of quantum-oscillation measurements. Moreover, our field-dependent measurements seem to be an excellent playground for a systematic study of Mott's formula in heavy-fermion systems and of transport signatures across Lifshitz transitions.

Within the third project, we studied the superconducting state of $\mathrm{LaPt}_{4} \mathrm{Ge}_{12}$. While previous NMR and photoemission studies on $\mathrm{LaPt}_{4} \mathrm{Ge}_{12}$ were interpreted in terms of a conventional BCS superconductor, more extensive studies on the closely related $\mathrm{PrPt}_{4} \mathrm{Ge}_{12}$ revealed signs of unconventional and/or multiband scenarios. Results on the series $\operatorname{La}_{x} \operatorname{Pr}_{1-x} \mathrm{Pt}_{4} \mathrm{Ge}_{12}$, however, indicate compatible order parameters for the two endmembers. To investigate the superconducting order parameter of $\mathrm{LaPt}_{4} \mathrm{Ge}_{12}$ in more detail, we performed thermalconductivity studies combined with specific-heat and resistivity measurements. We compared our experimental results with predictions for a fully gapped BCS superconductor and for superconductors with nodes in the gap.

Our specific-heat and our temperature- and field-dependent thermal-conductivity results on $\mathrm{LaPt}_{4} \mathrm{Ge}_{12}$ can be consistently described by a model of a fully gapped superconductor. The field-angle dependence of the thermal conductivity shows an unexpected rich oscillation pattern. Usually, oscillations are taken as a sign of nodes in the superconducting gap and are used to determine their position. Our studies, however, indicate a nodeless gap. With a detailed analysis, we were able to assign most of the frequencies to features other than nodal structures. Only the origin of one frequency remains unclear. Nevertheless, these results clearly indicate that a careful analysis of field-angle-dependent studies is indispensable for a reliable interpretation of the results.

These three diverse projects were all addressed with thermal transport as primary probe, 
using the magnetic field to tune the interactions in the systems. They show that thermal transport can be a very general and powerful tool that complements other transport and thermodynamic measurements, allowing us to investigate very fundamental physical laws in strongly correlated condensed matter. 



\section{Bibliography}

[Abl91] N. N. Ablyazov, M. Y. Kuchiev, and M. E. Raikh. Topological transition and its connection with the conductivity and thermopower anomalies in two-dimensional systems. In: Phys. Rev. B 44 (1991), p. 8802.

[Abr12] E. Abrahams and P. Wölfle. Critical quasiparticle theory applied to heavy fermion metals near an antiferromagnetic quantum phase transition. In: Proc. Natl. Acad. Sci. U. S. A. 109 (2012), p. 3238.

[Abr14] E. Abrahams, J. Schmalian, and P. Wölfle. Strong-coupling theory of heavyfermion criticality. In: Phys. Rev. B 90 (2014), p. 045105.

[Alt11] M. M. Altarawneh, N. Harrison, S. E. Sebastian, L. Balicas, P. H. Tobash, J. D. Thompson, F. Ronning, and E. D. Bauer. Sequential Spin Polarization of the Fermi Surface Pockets in URh $\mathrm{Si}_{2}$ and Its Implications for the Hidden Order. In: Phys. Rev. Lett. 106 (2011), p. 146403.

[Ama89] A. Amato, D. Jaccard, J. Sierro, P. Haen, P. Lejay, and J. Flouquet. Transport properties under magnetic fields of the heavy fermion system $\mathrm{CeRu}_{2} \mathrm{Si}_{2}$ and related compounds (Ce,La)Ru Si $_{2}$. In: J. Low Temp. Phys. 77 (1989), p. 195.

[And70] P. W. Anderson. A poor man's derivation of scaling laws for the Kondo problem. In: J. Phys. C: Solid State Phys. 3 (1970), p. 2436.

[Ann10] J. F. Annett. Superconductivity, Superfluids and Condensates. Oxford University Press, 2010.

[Aok01] H. Aoki, M. Takashita, N. Kimura, T. Terashima, S. Uji, T. Matsumoto, and Y. Onuki. New Features of the Metamagnetic Transition in CeRu $\mathrm{S}_{2} \mathrm{i}_{2}$ from the dHvA Effect Study under High Pressure. In: J. Phys. Soc. Jpn. 70 (2001), p. 774.

[Aok05] Y. Aoki, H. Sugawara, H. Hisatomo, and H. Sato. Novel Kondo Behaviors Realized in the Filled Skutterudite Structure. In: J. Phys. Soc. Jpn. 74 (2005), p. 209.

[Aok11] D. Aoki, C. Paulsen, T. D. Matsuda, L. Malone, G. Knebel, P. Haen, P. Lejay, R. Settai, Y. Ōnuki, and J. Flouquet. Pressure Evolution of the Magnetic Field induced Ferromagnetic Fluctuation through the Pseudo-Metamagnetism of $\mathrm{CeRu}_{2} \mathrm{Si}_{2}$. In: J. Phys. Soc. Jpn. 80 (2011), p. 053702.

[Aok93] H. Aoki, S. Uji, A. K. Albessard, and Y. Ōnuki. Transition of f-electron nature from itinerant to localized: Metamagnetic transition in $\mathrm{CeR}_{2} \mathrm{Si}_{2}$ studied via the de Haas-van Alphen effect. In: Phys. Rev. Lett. 71 (1993), p. 2110.

[Aok98] Y. Aoki, T. D. Matsuda, H. Sugawara, H. Sato, H. Ohkuni, R. Settai, Y. Ōnuki, E. Yamamoto, Y. Haga, A. V. Andreev, V. Sechovsky, L. Havela, H. Ikeda, and K. Miyake. Thermal properties of metamagnetic transition in heavy-fermion systems. In: J. Magn. Magn. Mater. 177-181 (1998). International Conference on Magnetism, p. 271. 
[Arn11] J. Arndt, O. Stockert, K. Schmalzl, E. Faulhaber, H. S. Jeevan, C. Geibel, W. Schmidt, M. Loewenhaupt, and F. Steglich. Spin Fluctuations in Normal State $\mathrm{CeCu}_{2} \mathrm{Si}_{2}$ on Approaching the Quantum Critical Point. In: Phys. Rev. Lett. 106 (2011), p. 246401.

[Ash76] N. W. Ashcroft and D. N. Mermin. Solid State Physics. Holt, Rinehart and Winston, 1976.

[Aub97] H. Aubin, K. Behnia, M. Ribault, R. Gagnon, and L. Taillefer. Angular Position of Nodes in the Superconducting Gap of YBCO. In: Phys. Rev. Lett. 78 (1997), p. 2624.

[Bar59] J. Bardeen, G. Rickayzen, and L. Tewordt. Theory of the Thermal Conductivity of Superconductors. In: Phys. Rev. 113 (1959), p. 982.

[Bau02] E. D. Bauer, N. A. Frederick, P.-C. Ho, V. S. Zapf, and M. B. Maple. Superconductivity and heavy fermion behavior in $\mathrm{PrOs}_{4} \mathrm{Sb}_{12}$. In: Phys. Rev. B 65 (2002), p. 100506.

[Bau07] J. Bauer and A. C. Hewson. Field-dependent quasiparticles in a strongly correlated local system. II. In: Phys. Rev. B 76 (2007), p. 035119.

[Bed86] J. G. Bednorz and K. A. Müller. Possible high-Tc superconductivity in the BaLa-Cu-O system. In: Z. Phys. B Con. Mat. 64 (1986), p. 189.

[Beh04] K. Behnia, D. Jaccard, and J. Flouquet. On the thermoelectricity of correlated electrons in the zero-temperature limit. In: J. Phys.: Condens. Matter 16 (2004), p. 5187.

[Bel04] R. Bel. "Transport d'entropie, thermoélectricité dans les supraconducteurs non conventionnels". PhD thesis. Université Paris VII, 2004.

[Ber12] M. Bercx and F. F. Assaad. Metamagnetism and Lifshitz transitions in models for heavy fermions. In: Phys. Rev. B 86 (2012), p. 075108.

[Ber64] G. J. V. D. Berg. "Chapter IV Anomalies in Dilute Metallic Solutions of Transition Elements". In: ed. by C. J. Gorter. Vol. 4. Progress in Low Temperature Physics. Elsevier, 1964, pp. 194-264.

[Bes85] M. Besnus, J. Kappler, P. Lehmann, and A. Meyer. Low temperature heat capacity, magnetization, resistivity of $\mathrm{CeR}_{2} \mathrm{Si}_{2}$, with $\mathrm{Y}$ or La substitution. In: Solid State Communications 55 (1985), p. 779.

[Bla76] F. J. Blatt, P. A. Schroeder, C. L. Foiles, and D. Greig. Thermoelectric Power of Metals. Plenum Press, New York, 1976.

[Bla92] Y. M. Blanter, A. V. Pantsulaya, and A. A. Varlamov. Thermoelectric power and topological transitions in quasi-two-dimensional electronic systems. In: Phys. Rev. B 45 (1992), p. 6267.

[Bla94] Y. M. Blanter, M. I. Kaganov, A. V. Pantsulaya, and A. A. Varlamov. The theory of electronic topological transitions. In: Phys. Rep. 245 (1994), p. 159.

[Blu09] S. Blundell. Magnetism in Condensed Matter. Oxford University Press, 2009.

[Boa03] E. Boaknin, M. A. Tanatar, J. Paglione, D. Hawthorn, F. Ronning, R. W. Hill, M. Sutherland, L. Taillefer, J. Sonier, S. M. Hayden, and J. W. Brill. Heat Conduction in the Vortex State of $\mathrm{NbSe}_{2}$ : Evidence for Multiband Superconductivity. In: Phys. Rev. Lett. 90 (2003), p. 117003. 
[Bou01] F. Bouquet, Y. Wang, R. A. Fisher, D. G. Hinks, J. D. Jorgensen, A. Junod, and N. E. Phillips. Phenomenological two-gap model for the specific heat of $M g B_{2}$. In: Europhys. Lett. 56 (2001), p. 856.

[Bou14] M. Boukahil, A. Pourret, G. Knebel, D. Aoki, Y. Ōnuki, and J. Flouquet. Lifshitz transition and metamagnetism: Thermoelectric studies of $\mathrm{CeRu_{2 }} S i_{2}$. In: Phys. Rev. B 90 (2014), p. 075127.

[Bra67] U. Brandt, W. Pesch, and L. Tewordt. Theory of the density of states of pure type-II superconductors in high magnetic fields. In: Z. Phys. 201 (1967), p. 209.

[Buc04] W. Buckel and R. Kleiner. Supraleitung. WILEY-VCH Verlag, 2004.

[Buh13] J. M. Buhmann and M. Sigrist. Thermoelectric effect of correlated metals: Bandstructure effects and the breakdown of Mott's formula. In: Phys. Rev. B 88 (2013), p. 115128.

[Car65] C. Caroli and M. Cyrot. Thermal conductivity in dirty superconducting alloys in high field. In: Phys. Kondens. Mater 4 (1965), p. 285.

[Cha12] L. S. S. Chandra, M. K. Chattopadhyay, and S. B. Roy. Critical current density and vortex pinning in the two gap superconductor $\mathrm{PrPt}_{4} \mathrm{Ge}_{12}$. In: Supercond. Sci. Technol. 25 (2012), p. 105009.

[Col01] P. Coleman, C. Pépin, Q. Si, and R. Ramazashvili. How do Fermi liquids get heavy and die? In: J. Phys.: Condens. Matter 13 (2001), R723.

[Col02] P. Coleman. Local moment physics in heavy electron systems. In: AIP Conf. Proc. 629 (2002), p. 79.

[Con93] M. A. Continentino. Universal behavior in heavy fermions. In: Phys. Rev. B 47 (1993), p. 11587.

[Cus03] J. Custers, P. Gegenwart, H. Wilhelm, K. Neumaier, Y. Tokiwa, O. Trovarelli, C. Geibel, F. Steglich, C. Pepin, and P. Coleman. The break-up of heavy electrons at a quantum critical point. In: Nature 424 (2003), p. 524.

[Cus04] J. Custers. "Quantum-Critical Behavior in the Heavy-Fermion Compounds $\mathrm{YbRh}_{2} \mathrm{Si}_{2}$ and $\mathrm{CeIn}_{3-\mathrm{x}} \mathrm{Sn}_{\mathrm{x}} "$. PhD thesis. TU Dresden, 2004.

[Cyr65] M. Cyrot. Excitations électroniques dans les supraconducteurs purs de 2ème espèce. In: Phys. Kondens. Mater 3 (1965), p. 374.

[Dao06a] R. Daou, C. Bergemann, and S. R. Julian. Continuous Evolution of the Fermi Surface of $\mathrm{CeRu}_{2} \mathrm{Si}_{2}$ across the Metamagnetic Transition. In: Phys. Rev. Lett. 96 (2006), p. 026401.

[Dao06b] R. Daou, C. Bergemann, and S. R. Julian. Spin dependence of the quasiparticle masses in $\mathrm{CeRu}_{2} \mathrm{Si}_{2}$. In: Physica B 378-380 (2006). Proceedings of the International Conference on Strongly Correlated Electron Systems SCES 2005, p. 807.

[Das12] T. Das, A. B. Vorontsov, I. Vekhter, and M. J. Graf. Role of the Fermi-Surface Anisotropy in Angle-Dependent Magnetic-Field Oscillations for Identifying the Energy-Gap Anisotropy of $A_{y} F_{2} S_{2}$ Superconductors. In: Phys. Rev. Lett. 109 (2012), p. 187006.

[Don77] S. Doniach. The Kondo lattice and weak antiferromagnetism. In: Physica B+C 91 (1977), p. 231. 
[Dou63] R. L. Douglass. Heat transport by spin waves in Yttrium Iron Garnet. In: Phys. Rev. 129 (1963), p. 1132.

[Dub63] L. Dubeck, P. Lindenfeld, E. A. Lynton, and H. Rohrer. Thermal Conductivity of Superconductors of the Second Kind. In: Phys. Rev. Lett. 10 (1963), p. 98.

[Dub64] L. Dubeck, P. Lindenfeld, E. A. Lynton, and H. Rohrer. Magnetic and Thermal Properties of Second-Kind Superconductors. II. Thermal Conductivity in the Mixed State. In: Rev. Mod. Phys. 36 (1964), p. 110.

[Dur00] A. C. Durst and P. A. Lee. Impurity-induced quasiparticle transport and universallimit Wiedemann-Franz violation in d-wave superconductors. In: Phys. Rev. B 62 (2000), p. 1270.

[Enn05] C. Enns and S. Hunklinger. Low-Temperature Physics. Springer, 2005.

[Ern11] S. Ernst, S. Kirchner, C. Krellner, C. Geibel, G. Zwicknagl, F. Steglich, and S. Wirth. Emerging local Kondo screening and spatial coherence in the heavyfermion metal $Y_{b R h_{2}} S i_{2}$. In: Nature 474 (2011), p. 362.

[Fer64] R. A. Ferrell. Temperature Dependence of the Superconductivity Energy Gap. In: Z. Phys. 182 (1964), p. 1.

[Fey63] R. Feynman, R. Leighton, and M. Sands. The Feynman Lectures on Physics Vol. 1. Addison-Wesley Pub. Co., 1963.

[Flo02] J. Flouquet, P. Haen, S. Raymond, D. Aoki, and G. Knebel. Itinerant metamagnetism of $\mathrm{CeRu}_{2} \mathrm{Si}_{2}$ : bringing out the dead. Comparison with the new $\mathrm{Sr}_{3} \mathrm{Ru}_{2} \mathrm{O}_{7}$ case. In: Physica B 319 (2002), p. 251.

[För] T. Förster et.al. to be published.

[Fra84] J. J. M. Franse, P. H. Frings, A. de Visser, A. Menovsky, T. T. M. Palstra, P. H. Kes, and J. A. Mydosh. Spin fluctuations and superconductivity in UPt $_{3}$. In: Physica B+C 126 (1984), p. 116.

[Fri09] S. Friedemann, T. Westerkamp, M. Brando, N. Oeschler, S. Wirth, P. Gegenwart, C. Krellner, C. Geibel, and F. Steglich. Detaching the antiferromagnetic quantum critical point from the Fermi-surface reconstruction in $\mathrm{YbRh}_{2} \mathrm{Si}_{2}$. In: Nat. Phys. 5 (2009), p. 465.

[Fri10] S. Friedemann, N. Oeschler, S. Wirth, C. Krellner, C. Geibel, F. Steglich, S. Paschen, S. Kirchner, and Q. Si. Fermi-surface collapse and dynamical scaling near a quantum-critical point. In: Proc. Natl. Acad. Sci. U. S. A. 107 (2010), p. 14547.

[Fri13] S. Friedemann, S. Paschen, C. Geibel, S. Wirth, F. Steglich, S. Kirchner, E. Abrahams, and Q. Si. Comment on "Zeeman-Driven Lifshitz Transition: A Model for the Experimentally Observed Fermi-Surface Reconstruction in YbRh ${ }_{2} S i_{2}$. In: Phys. Rev. Lett. 111 (2013), p. 139701.

[Frö36] H. Fröhlich and W. Heitler. Time effects in the magnetic cooling method. II. The conductivity of heat. In: Proc. Roy. Soc. A 155 (1936), p. 640.

[Geg02] P. Gegenwart, J. Custers, C. Geibel, K. Neumaier, T. Tayama, K. Tenya, O. Trovarelli, and F. Steglich. Magnetic-Field Induced Quantum Critical Point in

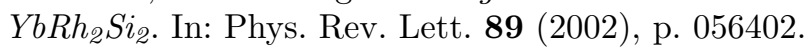


[Geg05] P. Gegenwart, J. Custers, Y. Tokiwa, C. Geibel, and F. Steglich. Ferromagnetic Quantum Critical Fluctuations in $\mathrm{YbRh}_{2}\left(\mathrm{Si}_{0.95} \mathrm{Ge}_{0.05}\right)_{2}$. In: Phys. Rev. Lett. 94 (2005), p. 076402.

[Geg06] P. Gegenwart, Y. Tokiwa, T. Westerkamp, F. Weickert, J. Custers, J. Ferstl, C. Krellner, C. Geibel, P. Kerschl, K.-H. Müller, and F. Steglich. High-field phase diagram of the heavy-fermion metal $\mathrm{YbRh}_{2} S i_{2}$. In: New J. Phys. 8 (2006), p. 171.

[Geg07] P. Gegenwart, T. Westerkamp, C. Krellner, Y. Tokiwa, S. Paschen, C. Geibel, F. Steglich, E. Abrahams, and Q. Si. Multiple Energy Scales at a Quantum Critical Point. In: Science 315 (2007), p. 969.

[Geg08] P. Gegenwart, T. Westerkamp, C. Krellner, M. Brando, Y. Tokiwa, C. Geibel, and F. Steglich. Unconventional quantum criticality in $\mathrm{YbRh}_{2} \mathrm{Si}_{2}$. In: Physica B 403 (2008). Proceedings of the International Conference on Strongly Correlated Electron Systems, p. 1184.

[Gol11] A. A. Golubov and A. E. Koshelev. Thermal conductivity in the mixed state of a superconductor at low magnetic fields. In: Phys. Rev. B 83 (2011), p. 094521.

[Gra96] M. J. Graf, S.-K. Yip, J. A. Sauls, and D. Rainer. Electronic thermal conductivity and the Wiedemann-Franz law for unconventional superconductors. In: Phys. Rev. B 53 (1996), p. 15147.

[Gum08] R. Gumeniuk, W. Schnelle, H. Rosner, M. Nicklas, A. Leithe-Jasper, and Y. Grin. Superconductivity in the Platinum Germanides $M_{4} t_{4} G_{12}$ ( $M=$ Rare-Earth or Alkaline-Earth Metal) with Filled Skutterudite Structure. In: Phys. Rev. Lett. 100 (2008), p. 017002.

[Gup72] A. K. Gupta and S. Wolf. Thermal Conductivity of Superconducting Lead-Indium Alloys. In: Phys. Rev. B 6 (1972), p. 2595.

[Haa34] W. J. de Haas, J. de Boer, and G. J. van dën Berg. The electrical resistance of gold, copper and lead at low temperatures. In: Physica 1 (1934), p. 1115.

[Hac11] A. Hackl and M. Vojta. Zeeman-Driven Lifshitz Transition: A Model for the Experimentally Observed Fermi-Surface Reconstruction in YbRh ${ }_{2} \mathrm{Si}_{2}$. In: Phys. Rev. Lett. 106 (2011), p. 137002.

[Hac13] A. Hackl and M. Vojta. Hackl and Vojta Reply: Zeeman-Driven Lifshitz Transition: A Model for the Experimentally Observed Fermi-Surface Reconstruction in $Y_{b R h_{2}} S i_{2}$. In: Phys. Rev. Lett. 111 (2013), p. 139702.

[Hae87] P. Haen, J. Flouquet, F. Lapierre, P. Lejay, and G. Remenyi. Metamagnetic-like transition in $C e R u_{2} S i_{2}$. In: J. Low Temp. Phys. 67 (1987), p. 391.

[Har10a] S. Hartmann. "Thermoelectric Transport in Correlated Electron Systems". PhD thesis. Technische Universität Dresden, 2010.

[Har10b] S. Hartmann, N. Oeschler, C. Krellner, C. Geibel, S. Paschen, and F. Steglich. Thermopower Evidence for an Abrupt Fermi Surface Change at the Quantum Critical Point of $\mathrm{YbRh}_{2} \mathrm{Si}_{2}$. In: Phys. Rev. Lett. 104 (2010), p. 096401.

[Her76] J. A. Hertz. Quantum critical phenomena. In: Phys. Rev. B 14 (1976), p. 1165.

[Hes01] C. Hess, C. Baumann, U. Ammerahl, B. Büchner, F. Heidrich-Meisner, W. Brenig, and A. Revcolevschi. Magnon heat transport in $(\mathrm{Sr}, \mathrm{Ca}, \mathrm{La})_{14} \mathrm{Cu}_{24} \mathrm{O}_{41}$. In: Phys. Rev. B 64 (2001), p. 184305. 
[Hes03] C. Hess, B. Büchner, U. Ammerahl, L. Colonescu, F. Heidrich-Meisner, W. Brenig, and A. Revcolevschi. Magnon Heat Transport in Doped $\mathrm{La}_{2} \mathrm{CuO}_{4}$. In: Phys. Rev. Lett. 90 (2003), p. 197002.

[Hes90] H. F. Hess, R. B. Robinson, and J. V. Waszczak. Vortex-core structure observed with a scanning tunneling microscope. In: Phys. Rev. Lett. 64 (1990), p. 2711.

[Hew04] A. Hewson, A. Oguri, and D. Meyer. Renormalized parameters for impurity models. In: Eur. Phys. J. B 40 (2004), p. 177.

[Hew06] A. C. Hewson, J. Bauer, and W. Koller. Field dependent quasiparticles in a strongly correlated local system. In: Phys. Rev. B 73 (2006), p. 045117.

[Hew93] A. C. Hewson. The Kondo Problem to Heavy Fermions. Cambridge University Press, Cambridge, England, 1993.

[Ish02] K. Ishida, K. Okamoto, Y. Kawasaki, Y. Kitaoka, O. Trovarelli, C. Geibel, and F. Steglich. YbRh ${ }_{2} S i_{2}$ : Spin Fluctuations in the Vicinity of a Quantum Critical Point at Low Magnetic Field. In: Phys. Rev. Lett. 89 (2002), p. 107202.

[Ish03] K. Ishida, D. E. MacLaughlin, B.-L. Young, K. Okamoto, Y. Kawasaki, Y. Kitaoka, G. J. Nieuwenhuys, R. H. Heffner, O. O. Bernal, W. Higemoto, A. Koda, R. Kadono, O. Trovarelli, C. Geibel, and F. Steglich. Low-temperature magnetic order and spin dynamics in YbRh ${ }_{2} S i_{2}$. In: Phys. Rev. B 68 (2003), p. 184401.

[Iza01] K. Izawa, H. Takahashi, H. Yamaguchi, Y. Matsuda, M. Suzuki, T. Sasaki, T. Fukase, Y. Yoshida, R. Settai, and Y. Onuki. Superconducting Gap Structure of Spin-Triplet Superconductor $\mathrm{Sr}_{2} \mathrm{RuO}_{4}$ Studied by Thermal Conductivity. In: Phys. Rev. Lett. 86 (2001), p. 2653.

[Kad03] H. Kadowaki, B. Fåk, T. Fukuhara, K. Maezawa, K. Nakajima, M. A. Adams, S. Raymond, and J. Flouquet. Spin fluctuations and non-Fermi-liquid behavior of $\mathrm{CeNi}_{2} \mathrm{Ge}_{2}$. In: Phys. Rev. B 68 (2003), p. 140402.

[Kad86] K. Kadowaki and S. B. Woods. Universal relationship of the resistivity and specific heat in heavy-Fermion compounds. In: Solid State Commun. 58 (1986), p. 507.

[Kam96] S. Kambe, J. Flouquet, P. Haen, and P. Lejay. Characteristic of metamagnetic transition in $\mathrm{CeRu}_{2} \mathrm{Si}_{2}$ revealed with hall mobility. In: J. Low Temp. Phys. 102 (1996), p. 477.

[Kan10] F. Kanetake, H. Mukuda, Y. Kitaoka, K.-I. Magishi, H. Sugawara, K. M. Itoh, and E. E. Haller. Superconducting Characteristics of Filled Skutterudites $\mathrm{LaPt}_{4} \mathrm{Ge}_{12}$ and $\mathrm{PrPt}_{4} \mathrm{Ge}_{12}:{ }^{73} \mathrm{Ge}-\mathrm{NQR} / \mathrm{NMR}$ Studies. In: J. Phys. Soc. Jpn. 79 (2010), p. 063702.

[Kno88] G. Knopp, A. Loidl, R. Caspary, U. Gottwick, C. Bredl, H. Spille, F. Steglich, and A. Murani. Specific heat, resistivity and neutron scattering studies in the Kondo lattice $\mathrm{CeNi}_{2} \mathrm{Ge}_{2}$. In: J. Magn. Magn. Mater. 74 (1988), p. 341.

[Köh08] U. Köhler, N. Oeschler, F. Steglich, S. Maquilon, and Z. Fisk. Energy scales of $L u_{1-x} \mathrm{Yb}_{x} R h_{2} S i_{2}$ by means of thermopower investigations. In: Phys. Rev. B 77 (2008), p. 104412.

[Kon64] J. Kondo. Resistance Minimum in Dilute Magnetic Alloys. In: Prog. Theor. Phys. 32 (1964), p. 37. 
[Kor06] K. Kordonis, A. V. Sologubenko, T. Lorenz, S.-W. Cheong, and A. Freimuth. Spin Thermal Conductivity of the Haldane Chain Compound $\mathrm{Y}_{2} \mathrm{BaNiO}$. In: Phys. Rev. Lett. 97 (2006), p. 115901.

[Kos04] A. M. Kosevich. Topology and solid-state physics (Review). In: Low Temp. Phys. 30 (2004), p. 97.

[Kot11] H. Kotegawa, V. Taufour, D. Aoki, G. Knebel, and J. Flouquet. Evolution toward Quantum Critical End Point in UGe.2. In: J. Phys. Soc. Jpn. 80 (2011), p. 083703.

[Kre09] C. Krellner, S. Hartmann, A. Pikul, N. Oeschler, J. G. Donath, C. Geibel, F. Steglich, and J. Wosnitza. Violation of Critical Universality at the Antiferromagnetic Phase Transition of $Y b R h_{2} S i_{2}$. In: Phys. Rev. Lett. 102 (2009), p. 196402.

[Küb98] C. Kübert and P. J. Hirschfeld. Quasiparticle Transport Properties of d-Wave Superconductors in the Vortex State. In: Phys. Rev. Lett. 80 (1998), p. 4963.

[Küc03] R. Küchler, N. Oeschler, P. Gegenwart, T. Cichorek, K. Neumaier, O. Tegus, C. Geibel, J. A. Mydosh, F. Steglich, L. Zhu, and Q. Si. Divergence of the Grüneisen Ratio at Quantum Critical Points in Heavy Fermion Metals. In: Phys. Rev. Lett. 91 (2003), p. 066405.

[Kus02] H. Kusunose, T. M. Rice, and M. Sigrist. Electronic thermal conductivity of multigap superconductors: Application to $M g B_{2}$. In: Phys. Rev. B 66 (2002), p. 214503.

[LeB11] D. LeBoeuf, N. Doiron-Leyraud, B. Vignolle, M. Sutherland, B. J. Ramshaw, J. Levallois, R. Daou, F. Laliberte, O. Cyr-Choiniere, J. Chang, Y. J. Jo, L. Balicas, R. Liang, D. A. Bonn, W. N. Hardy, C. Proust, and L. Taillefer. Lifshitz critical point in the cuprate superconductor $\mathrm{YBa}_{2} \mathrm{Cu}_{3} \mathrm{O}_{y}$ from high-field Hall effect measurements. In: Phys. Rev. B 83 (2011), p. 054506.

[Lif60] I. Lifshitz. Anomalies of the electron characteristics of a metal in the high pressure region. In: Sov. Phys. JETP-USSR 11 (1960), p. 1130.

[Liu10] C. Liu, T. Kondo, R. M. Fernandes, A. D. Palczewski, E. D. Mun, N. Ni, A. N. Thaler, A. Bostwick, E. Rotenberg, J. Schmalian, S. L. Budko, P. C. Canfield, and A. Kaminski. Evidence for a Lifshitz transition in electron-doped iron arsenic superconductors at the onset of superconductivity. In: Nat. Phys. 6 (2010), p. 419.

[Löh07] H. v. Löhneysen, A. Rosch, M. Vojta, and P. Wölfle. Fermi-liquid instabilities at magnetic quantum phase transitions. In: Rev. Mod. Phys. 79 (2007), p. 1015.

[Löh93] H. Löhneysen, H. Schlager, and A. Schroder. Magnetic correlations and magnetic ordering in $\mathrm{CeCu}_{6-x} A u_{x}$ single crystals. In: Physica B 186-188 (1993), p. 590.

[Low70] J. Lowell and J. Sousa. Mixed-state thermal conductivity of type II superconductors. In: J. Low Temp. Phys. 3 (1970), p. 65.

[Mac12] Y. Machida, A. Itoh, Y. So, K. Izawa, Y. Haga, E. Yamamoto, N. Kimura, Y. Onuki, Y. Tsutsumi, and K. Machida. Twofold Spontaneous Symmetry Breaking in the Heavy-Fermion Superconductor UPt. In: Phys. Rev. Lett. 108 (2012), p. 157002 .

[Mac13] Y. Machida, K. Tomokuni, K. Izawa, G. Lapertot, G. Knebel, J.-P. Brison, and J. Flouquet. Verification of the Wiedemann-Franz Law in $\mathrm{YbRh}_{2} \mathrm{Si}_{2}$ at a Quantum Critical Point. In: Phys. Rev. Lett. 110 (2013), p. 236402. 
[Mah13] R. Mahajan, M. Barkeshli, and S. A. Hartnoll. Non-Fermi liquids and the Wiedemann-Franz law. In: Phys. Rev. B 88 (2013), p. 125107.

[Mai10] A. Maisuradze, W. Schnelle, R. Khasanov, R. Gumeniuk, M. Nicklas, H. Rosner, A. Leithe-Jasper, Y. Grin, A. Amato, and P. Thalmeier. Evidence for timereversal symmetry breaking in superconducting $\mathrm{PrPt}_{4} \mathrm{Ge}_{12}$. In: Phys. Rev. B 82 (2010), p. 024524.

[Mak67] K. Maki. Thermal Conductivity of Pure Type-II Superconductors in High Magnetic Fields. In: Phys. Rev. 158 (1967), p. 397.

[Mal11] L. Malone, T. D. Matusda, A. Antunes, G. Knebel, V. Taufour, D. Aoki, K. Behnia, C. Proust, and J. Flouquet. Thermoelectric evidence for high-field anoma-

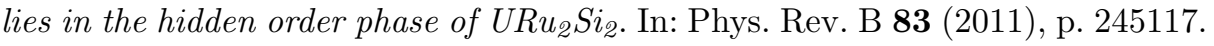

[Mat06] Y. Matsuda, K. Izawa, and I. Vekhter. Nodal structure of unconventional superconductors probed by angle resolved thermal transport measurements. In: J. Phys.: Condens. Matter 18 (2006), R705.

[Mat14] Y. Matsumoto, K. Kuga, Y. Karaki, Y. Shimura, T. Sakakibara, M. Tokunaga, K. Kindo, and S. Nakatsuji. Field evolution of quantum critical and heavy Fermi-liquid components in the magnetization of the mixed valence compound $\beta-\mathrm{YbAlB}_{4}$. arXiv:1407.6142. 2014.

[Mer14] P. Merchant, B. Normand, K. W. Krämer, M. Boehm, D. F. McMorrow, and C. Rüegg. Quantum and classical criticality in a dimerized quantum antiferromagnet. In: Nat. Phys. (2014).

[Mil02] A. J. Millis, A. J. Schofield, G. G. Lonzarich, and S. A. Grigera. Metamagnetic Quantum Criticality in Metals. In: Phys. Rev. Lett. 88 (2002), p. 217204.

[Mil93] A. J. Millis. Effect of a nonzero temperature on quantum critical points in itinerant fermion systems. In: Phys. Rev. B 48 (1993), p. 7183.

[Min99] V. P. Mineev and K. V. Samokhin. Introduction to Unconventional Superconductivity. Gordon and Breach Science Publisher, 1999.

[Miy02] K. Miyake and O. Narikiyo. Enhanced Impurity Scattering due to Quantum Critical Fluctuations: Perturbational Approach. In: J. Phys. Soc. Jpn. 71 (2002), p. 867.

[Mor84] K. Mori, Y. Isikawa, and K. Sato. Thermal Conductivity and Electrical Resistivity of Ferromagnetic Intermetallic Compound ErNi. In: J. Phys. Soc. Jpn. 53 (1984), p. 664.

[Mor95] T. Moriya and T. Takimoto. Anomalous Properties around Magnetic Instability in Heavy Electron Systems. In: J. Phys. Soc. Jpn. 64 (1995), p. 960.

[Mot71] N. F. Mott and E. A. Davis. Electronic processes in non-crystalline materials. Oxford Univ. Press, 1971.

[Müh59] B. Mühlschlegel. Die thermischen Funktionen des Spraleiters. In: Z. Phys. 155 (1959), p. 313.

[Mut68] Y. Muto, K. Noto, T. Mamiya, and T. Fukuroi. The Thermal Conductivity of the Type II Superconductors; In-Pb Alloys. II. Comparison with the Caroli-Cyrot Theory. In: J. Phys. Soc. Jpn. 24 (1968), p. 992. 
[Nak12] Y. Nakamura, H. Okazaki, R. Yoshida, T. Wakita, H. Takeya, K. Hirata, M. Hirai, Y. Muraoka, and T. Yokoya. Comparative photoemission studies on the superconducting gap of the filled skutterudite superconductors $\mathrm{LaPt}_{4} \mathrm{Ge}_{12}$ and $\mathrm{PrPt}_{4} \mathrm{Ge}_{12}$. In: Phys. Rev. B 86 (2012), p. 014521.

[Nar13] H. R. Naren, S. Friedemann, G. Zwicknagl, C. Krellner, C. Geibel, F. Steglich, and S. Wirth. Lifshitz transitions and quasiparticle de-renormalization in $Y b R h_{2} S i_{2}$. In: New J. Phys. 15 (2013), p. 093032.

[Oda94] K. Oda, T. Kumada, K. Sugiyama, N. Sato, T. Komatubara, and M. Date. High Field Magnetization of $U P d_{2} A l_{3}$. In: J. Phys. Soc. Jpn. 63 (1994), p. 3115.

[Oes08] N. Oeschler, S. Hartmann, A. Pikul, C. Krellner, C. Geibel, and F. Steglich. Lowtemperature specific heat of $Y b R h_{2} S i_{2}$. In: Physica B 403 (2008). Proceedings of the International Conference on Strongly Correlated Electron Systems, p. 1254.

[Pas04] S. Paschen, T. Lühmann, S. Wirth, P. Gegenwart, O. Trovarelli, C. Geibel, F. Steglich, P. Coleman, and Q. Si. Hall-effect evolution across a heavy-fermion quantum critical point. In: Nature 432 (2004), p. 881.

[Pes74] W. Pesch, R. Watts-Tobin, and L. Kramer. Transport coefficients in the mixed state of dirty superconductors. In: Z. Phys. 269 (1974), p. 253.

[Pet06] R. Peters, T. Pruschke, and F. B. Anders. Numerical renormalization group approach to Green's functions for quantum impurity models. In: Phys. Rev. B 74 (2006), p. 245114.

[Pfa12a] H. Pfau, R. Daou, M. Brando, and F. Steglich. Thermoelectric transport across the metamagnetic transition of $\mathrm{CeRu}_{2} \mathrm{Si}_{2}$. In: Phys. Rev. B 85 (2012), p. 035127.

[Pfa12b] H. Pfau, S. Hartmann, U. Stockert, P. Sun, S. Lausberg, M. Brando, S. Friedemann, C. Krellner, C. Geibel, S. Wirth, S. Kirchner, E. Abrahams, Q. Si, and F. Steglich. Thermal and electrical transport across a magnetic quantum critical point. In: Nature 484 (2012), p. 493.

[Pfa13] H. Pfau, R. Daou, S. Lausberg, H. R. Naren, M. Brando, S. Friedemann, S. Wirth, T. Westerkamp, U. Stockert, P. Gegenwart, C. Krellner, C. Geibel, G. Zwicknagl, and F. Steglich. Interplay between Kondo Suppression and Lifshitz Transitions in $\mathrm{YbRh}_{2} \mathrm{Si}_{2}$ at High Magnetic Fields. In: Phys. Rev. Lett. 110 (2013), p. 256403.

[Poo00] C. P. J. Poole. Handbook of Superconductivity. Academic Press San Diego, 2000.

[Pou13] A. Pourret, G. Knebel, T. D. Matsuda, G. Lapertot, and J. Flouquet. Magnetic Polarization and Fermi Surface Instability: Case of $\mathrm{YbRh}_{2} \mathrm{Si}_{2}$. In: J. Phys. Soc. Jpn. 82 (2013), p. 053704.

[Pou14] A. Pourret, D. Aoki, M. Boukahil, J.-P. Brison, W. Knafo, G. Knebel, S. Raymond, M. Taupin, Y. Ōnuki, and J. Flouquet. Quantum Criticality and Lifshitz

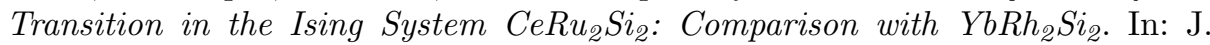
Phys. Soc. Jpn. 83 (2014), p. 061002.

[Pro02] C. Proust, E. Boaknin, R. W. Hill, L. Taillefer, and A. P. Mackenzie. Heat Transport in a Strongly Overdoped Cuprate: Fermi Liquid and a Pure d-Wave BCS Superconductor. In: Phys. Rev. Lett. 89 (2002), p. 147003.

[Qua99a] QuantumDesign. Physical Property Measurement System - Hardware Manual. Quantum Design. 1999. 
[Qua99b] QuantumDesign. Physical Property Measurement System - Heat Capacity Option User's Manual. Quantum Design. 1999.

[Qua99c] QuantumDesign. Physical Property Measurement System - Thermal Transport Option User's Manual. Quantum Design. 1999.

[Rei14] J.-P. Reid, M. A. Tanatar, R. Daou, R. Hu, C. Petrovic, and L. Taillefer. Wiedemann-Franz law and nonvanishing temperature scale across the field-tuned quantum critical point of $Y_{b R h_{2}} S i_{2}$. In: Phys. Rev. B 89 (2014), p. 045130.

[Rou08] P. M. C. Rourke, A. McCollam, G. Lapertot, G. Knebel, J. Flouquet, and S. R. Julian. Magnetic-Field Dependence of the $\mathrm{YbRh}_{2} \mathrm{Si}_{2}$ Fermi Surface. In: Phys. Rev. Lett. 101 (2008), p. 237205.

[Sam76] K. Samwer and K. Winzer. Magnetoresistivity of the Kondo-system (La,Ce)B . In: Z. Phys. B Con. Mat. 25 (1976), p. 269.

[Sat04] M. Sato, Y. Koike, S. Katano, N. Metoki, H. Kadowaki, and S. Kawarazaki. Fieldinduced Ferromagnetic Correlation in the Metamagnetic Crossover in $\mathrm{CeRu}_{2} \mathrm{Si}_{2}$ as Studied by Neutron Scattering. In: J. Phys. Soc. Jpn. 73 (2004), p. 3418.

[Sch09a] U. Schaufuß, V. Kataev, A. A. Zvyagin, B. Büchner, J. Sichelschmidt, J. Wykhoff, C. Krellner, C. Geibel, and F. Steglich. Evolution of the Kondo State of $Y b R h_{2} S i_{2}$ Probed by High-Field ESR. In: Phys. Rev. Lett. 102 (2009), p. 076405.

[Sch09b] E. Schuberth, M. Tippmann, M. Kath, C. Krellner, C. Geibel, T. Westerkamp, C. Klingner, and F. Steglich. Magnetization measurements on $\mathrm{YbRh}_{2} \mathrm{Si}_{2}$ at very low temperatures. In: J. Phys. Conf. Ser. 150 (2009), p. 042178.

[Sch70] E. Schmidbauer, H. Wenzl, and E. Umlauf. Wärmeleitung von reinen Typ II Supraleitern im Magnetfeld. In: Z. Phys. 240 (1970), p. 30.

[Sec86] V. Sechovsky, L. Havela, F. de Boer, J. Franse, P. Veenhuizen, J. Sebek, J. Stehno, and A. Andreev. Systematics across the UTX series ( T = Ru, Co, Ni; X $=A l, G a, S n)$ of high-field and low-temperature properties of non-ferromagnetic compounds. In: Physica B+C 142 (1986), p. 283.

[Ser97] M. Sera, S. Kobayashi, M. Hiroi, N. Kobayashi, H. Ohkuni, and Y. Ōnuki. Ther-

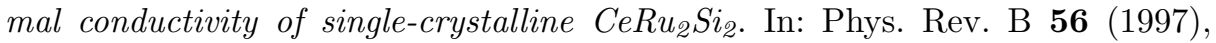
p. 13689.

[Sey06a] G. Seyfarth. "Multiband Superconductivity in the Heavy Fermion compounds $\mathrm{PrOs}_{4} \mathrm{Sb}_{12}$ and CeCoIn 5 ". PhD thesis. Université Joseph Fourier Grenoble, 2006.

[Sey06b] G. Seyfarth, J. P. Brison, M.-A. Méasson, D. Braithwaite, G. Lapertot, and J. Flouquet. Superconducting $\mathrm{PrOs}_{4} \mathrm{Sb}_{12}$ : A Thermal Conductivity Study. In: Phys. Rev. Lett. 97 (2006), p. 236403.

[Sha09] H. Shakeripour, C. Petrovic, and L. Taillefer. Heat transport as a probe of superconducting gap structure. In: New J. Phys. 11 (2009), p. 055065.

[Si01] Q. Si, S. Rabello, K. Ingersent, and J. L. Smith. Locally critical quantum phase transitions in strongly correlated metals. In: Nature 413 (2001), p. 804.

[Si11] Q. Si. "Understanding Quantum Phase Transitions". In: ed. by L. D. Carr. CRC Press, 2011. Chap. Quantum Criticality and the Kondo Lattice, pp. 193-216. 
[Si13] Q. Si and S. Paschen. Quantum phase transitions in heavy fermion metals and Kondo insulators. In: Phys. Status Solidi B 250 (2013), p. 425.

[Sol00] A. V. Sologubenko, K. Giannó, H. R. Ott, U. Ammerahl, and A. Revcolevschi. Thermal conductivity of the hole-doped spin ladder system $\mathrm{Sr}_{14-x} \mathrm{Ca}_{x} \mathrm{Cu}_{24} \mathrm{O}_{41}$. In: Phys. Rev. Lett. 84 (2000), p. 2714.

[Sol02] A. V. Sologubenko, J. Jun, S. M. Kazakov, J. Karpinski, and H. R. Ott. Thermal conductivity of single-crystalline $M g B_{2}$. In: Phys. Rev. B 66 (2002), p. 014504.

[Sol08] A. V. Sologubenko, T. Lorenz, J. A. Mydosh, A. Rosch, K. C. Shortsleeves, and M. M. Turnbull. Field-Dependent Thermal Transport in the Haldane Chain Compound NENP. In: Phys. Rev. Lett. 100 (2008), p. 137202.

[Ste01] G. R. Stewart. Non-Fermi-liquid behavior in d-and f-electron metals. In: Rev. Mod. Phys. 73 (2001), p. 797.

[Ste14] F. Steglich, H. Pfau, S. Lausberg, S. Hamann, P. Sun, U. Stockert, M. Brando, S. Friedemann, C. Krellner, C. Geibel, S. Wirth, S. Kirchner, E. Abrahams, and Q. Si. Evidence of a Kondo Destroying Quantum Critical Point in YbRh ${ }_{2} \mathrm{Si}_{2}$. In: J. Phys. Soc. Jpn. 83 (2014), p. 061001.

[Ste79] F. Steglich, J. Aarts, C. D. Bredl, W. Lieke, D. Meschede, W. Franz, and H. Schäfer. Superconductivity in the Presence of Strong Pauli Paramagnetism: $\mathrm{CeCu}_{2} \mathrm{Si}_{2}$. In: Phys. Rev. Lett. 43 (1979), p. 1892.

[Sto04] O. Stockert, E. Faulhaber, G. Zwicknagl, N. Stüßer, H. S. Jeevan, M. Deppe, R. Borth, R. Küchler, M. Loewenhaupt, C. Geibel, and F. Steglich. Nature of the A Phase in $\mathrm{CeCu}_{2} \mathrm{Si}_{2}$. In: Phys. Rev. Lett. 92 (2004), p. 136401.

[Sto06] O. Stockert, M. Koza, J. Ferstl, A. Murani, C. Geibel, and F. Steglich. Crystalline electric field excitations of the non-Fermi-liquid. In: Physica B 378-380 (2006). Proceedings of the International Conference on Strongly Correlated Electron Systems SCES 2005, p. 157.

[Sto11] O. Stockert and F. Steglich. Unconventional Quantum Criticality in Heavy-Fermion Compounds. In: Annu. Rev. Condens. Matter 2 (2011), p. 79.

[Sto12a] C. Stock, C. Broholm, F. Demmel, J. Van Duijn, J. W. Taylor, H. J. Kang, R. Hu, and C. Petrovic. From Incommensurate Correlations to Mesoscopic Spin Resonance in $\mathrm{YbRh}_{2} S i_{2}$. In: Phys. Rev. Lett. 109 (2012), p. 127201.

[Sto12b] O. Stockert, S. Kirchner, F. Steglich, and Q. Si. Superconductivity in Ce- and U-Based "122" Heavy-Fermion Compounds. In: J. Phys. Soc. Jpn. 81 (2012), p. 011001.

[Sug90] K. Sugiyama, H. Fuke, K. Kindo, K. Shimohata, A. A. Menovsky, J. A. Mydosh, and M. Date. Field-Induced Destruction of Heavy Fermion State in URu $u_{2} S_{2}$. In: J. Phys. Soc. Jpn. 59 (1990), p. 3331.

[Sum86] A. Sumiyama, Y. Oda, H. Nagano, Y. Ōnuki, K. Shibutani, and T. Komatsubara. Coherent Kondo State in a Dense Kondo Substance: $C e_{x} L a_{1-x} C u_{6}$. In: J. Phys. Soc. Jpn. 55 (1986), p. 1294.

[Tak96] M. Takashita, H. Aoki, T. Terashima, S. Uji, K. Maezawa, R. Settai, and Y. Ōnuki. dHvA Effect Study of Metamagnetic Transition in CeRu $\mathrm{S}_{2} \mathrm{i}_{2}$ - The State above the Metamagnetic Transition. In: J. Phys. Soc. Jpn. 65 (1996), p. 515. 
[Tan07] M. A. Tanatar, J. Paglione, C. Petrovic, and L. Taillefer. Anisotropic Violation of the Wiedemann-Franz Law at a Quantum Critical Point. In: Science 316 (2007), p. 1320 .

[Tau10] V. Taufour, D. Aoki, G. Knebel, and J. Flouquet. Tricritical Point and Wing Structure in the Itinerant Ferromagnet UGe. In: Phys. Rev. Lett. 105 (2010), p. 217201.

[Tau13] M. Taupin. "Study of the magnetic heavy fermions UCoGe and $\mathrm{YbRh}_{2} \mathrm{Si}_{2}$ by transport measurements". PhD thesis. Universite Joseph-Fourier - Grenoble I, 2013.

[Tew89] L. Tewordt and T. Wölkhausen. Theory of thermal conductivity of the lattice for high-Tc superconductors. In: Solid State Commun. 70 (1989), p. 839.

[Tin96] M. Tinkham. Introduction to Superconductivity. Ed. by E. C. J. Shira. McGrawHill Inc., 1996.

[Tod08] M. Toda, H. Sugawara, K.-i. Magishi, T. Saito, K. Koyama, Y. Aoki, and H. Sato. Electrical, Magnetic and NMR Studies of Ge-Based Filled Skutterudites $R t_{4} G e_{12}(R=L a, C e, P r, N d)$. In: J. Phys. Soc. Jpn. 77 (2008), p. 124702.

[Tok04] Y. Tokiwa, P. Gegenwart, F. Weickert, R. Küchler, J. Custers, J. Ferstl, C. Geibel, and F. Steglich. Suppression of the Kondo state in $\mathrm{YbRh}_{2} \mathrm{Si}_{2}$ by large magnetic fields. In: J. Magn. Magn. Mater. 272-276, Supplement (2004). Proceedings of the International Conference on Magnetism (ICM 2003), E87.

[Tok05] Y. Tokiwa, P. Gegenwart, T. Radu, J. Ferstl, G. Sparn, C. Geibel, and F. Steglich. Field-Induced Suppression of the Heavy-Fermion State in YbRh ${ }_{2} S i_{2}$. In: Phys. Rev. Lett. 94 (2005), p. 226402.

[Tok09] Y. Tokiwa, T. Radu, C. Geibel, F. Steglich, and P. Gegenwart. Divergence of the Magnetic Grüneisen Ratio at the Field-Induced Quantum Critical Point in $\mathrm{YbRh}_{2} \mathrm{Si}_{2}$. In: Phys. Rev. Lett. 102 (2009), p. 066401.

[Tom11] K. Tomokuni, Y. Machida, K. Izawa, G. Lapertot, G. Knebel, J.-P. Brison, and J. Flouquet. Thermal Transport Properties and Quantum Criticality of Heavy Fermion YbRh ${ }_{2} S i_{2}$. In: J. Phys. Soc. Jpn. 80 (2011), SA096.

[Tou70] Y. S. Touloukian, R. W. Powell, C. Y. Ho, and P. G. Klemens. Thermophysical Properties of Matter - Thermal Conductivity. Vol. 1-3. IFI/Plenum New YorkWashington, 1970.

[Tro00] O. Trovarelli, C. Geibel, S. Mederle, C. Langhammer, F. M. Grosche, P. Gegenwart, M. Lang, G. Sparn, and F. Steglich. YbRh ${ }_{2} S_{2}$ : Pronounced Non-FermiLiquid Effects above a Low-Lying Magnetic Phase Transition. In: Phys. Rev. Lett. 85 (2000), p. 626.

[Uhl04] M. Uhlarz, C. Pfleiderer, and S. M. Hayden. Quantum Phase Transitions in the Itinerant Ferromagnet $Z r Z n_{2}$. In: Phys. Rev. Lett. 93 (2004), p. 256404.

[Var89] A. Varlamov, V. Egorov, and A. Pantsulaya. Kinetic properties of metals near electronic topological transitions (2 1/2-order transitions). In: Adv. Phys. 38 (1989), p. 469. 
[Vek99a] I. Vekhter, P. J. Hirschfeld, J. P. Carbotte, and E. J. Nicol. Anisotropic thermodynamics of d-wave superconductors in the vortex state. In: Phys. Rev. B 59 (1999), R9023.

[Vek99b] I. Vekhter and A. Houghton. Quasiparticle Thermal Conductivity in the Vortex State of High- $T_{c}$ Cuprates. In: Phys. Rev. Lett. 83 (1999), p. 4626.

[Vie11] H. Vieyra. "Resistivity and Thermal Conductivity Measurements on Heavy Fermion Superconductors in Rotating Magnetic Fields". PhD thesis. Technische Universität Dresden, 2011.

[Vis98] A. de Visser, R. Keizer, M. Graf, A. Menovsky, and J. Franse. On the interplay of small-moment magnetism and superconductivity in UPt. In: J. Magn. Magn. Mater. 177-181, Part 1 (1998). International Conference on Magnetism, p. 287.

[Voj03] M. Vojta. Quantum phase transitions. In: Rep. Prog. Phys. 66 (2003), p. 2069.

[Vol93] G. Volovik. Superconductivity with lines of gap nodes: density of states in the vortex. In: JETP Lett. 58 (1993), p. 469.

[Vor06] A. Vorontsov and I. Vekhter. Nodal Structure of Quasi-Two-Dimensional Superconductors Probed by a Magnetic Field. In: Phys. Rev. Lett. 96 (2006), p. 237001.

[Wat04] T. Watanabe, K. Izawa, Y. Kasahara, Y. Haga, Y. Ōnuki, P. Thalmeier, K. Maki, and Y. Matsuda. Superconducting gap function in antiferromagnetic heavyfermion $\mathrm{UPd}_{2} \mathrm{Al}_{3}$ probed by angle-resolved magnetothermal transport measurements. In: Phys. Rev. B 70 (2004), p. 184502.

[Wei10] F. Weickert, M. Brando, F. Steglich, P. Gegenwart, and M. Garst. Universal signatures of the metamagnetic quantum critical endpoint: Application to CeRu $\mathrm{Si}_{2}$. In: Phys. Rev. B 81 (2010), p. 134438.

[Wer67] S. A. Werner, A. Arrott, and H. Kendrick. Temperature and Magnetic-Field Dependence of the Antiferromagnetism in Pure Chromium. In: Phys. Rev. 155 (1967), p. 528.

[Wet08] T. Wetserkamp. "Quantenphasenübergänge in den Schwere-Fermionen-Systemen $\mathrm{Yb}\left(\mathrm{Rh}_{1-\mathrm{x}} \mathrm{M}_{\mathrm{x}}\right)_{2} \mathrm{Si}_{2}$ und $\mathrm{CePd}_{1-\mathrm{x}} \mathrm{Rh}_{\mathrm{x}}$ ". PhD thesis. Technischen Universität Dresden, 2008.

[Wil75] K. G. Wilson. The renormalization group: Critical phenomena and the Kondo problem. In: Rev. Mod. Phys. 47 (1975), p. 773.

[Wil83a] M. C. K. Wiltshire and M. M. Elcombe. Temperature dependence of magnons in $\gamma-M n C u$. In: J. Magn. Magn. Mat. 31-34 (1983), p. 127.

[Wil83b] M. C. K. Wiltshire, M. M. Elcombe, and C. J. Howard. Temperature dependence of the magnetic excitations in $\gamma-M n_{90} C u_{10}$. In: J. Phys. F: Met. Phys. 15 (1983), p. 1595.

[Wöl11] P. Wölfle and E. Abrahams. Quasiparticles beyond the Fermi liquid and heavy fermion criticality. In: Phys. Rev. B 84 (2011), p. 041101.

[Won01] H. Won and K. Maki. Quasi-particle transport in vortex state of nodal superconductors. In: Curr. Appl. Phys. 1 (2001), p. 291. 
[Yam07] Y. Yamaji, T. Misawa, and M. Imada. Quantum Metamagnetic Transitions Induced by Changes in Fermi-Surface Topology: Applications to a Weak ItinerantElectron Ferromagnet ZrZn 2 . In: J. Phys. Soc. Jpn. 76 (2007), p. 063702.

[Yel11] E. A. Yelland, J. M. Barraclough, W. Wang, K. V. Kamenev, and A. D. Huxley. High-field superconductivity at an electronic topological transition in URhGe. In: Nat. Phys. 7 (2011), p. 890.

[Yel72] W. B. Yelon and L. Berger. Magnon Heat Conduction and Magnon Scattering Processes in Fe-Ni Alloys. In: Phys. Rev. B 6 (1972), p. 1974.

[Yu95] F. Yu, M. B. Salamon, A. J. Leggett, W. C. Lee, and D. M. Ginsberg. Tensor Magnetothermal Resistance in $\mathrm{YBa}_{2} \mathrm{Cu}_{3} \mathrm{O}_{7}$ via Andreev Scattering of Quasiparticles. In: Phys. Rev. Lett. 74 (1995), p. 5136.

[Zha13] J. L. Zhang, Y. Chen, L. Jiao, R. Gumeniuk, M. Nicklas, Y. H. Chen, L. Yang, B. H. Fu, W. Schnelle, H. Rosner, A. Leithe-Jasper, Y. Grin, F. Steglich, and H. Q. Yuan. Multiband superconductivity in $\mathrm{PrPt}_{4} \mathrm{Ge}_{12}$ single crystals. In: Phys. Rev. B 87 (2013), p. 064502.

[Zhu03] L. Zhu, M. Garst, A. Rosch, and Q. Si. Universally Diverging Grüneisen Parameter and the Magnetocaloric Effect Close to Quantum Critical Points. In: Phys. Rev. Lett. 91 (2003), p. 066404.

[Zim60] J. M. Ziman. Electrons and Phonons. Clarendon Press, Oxford, 1960.

[Zot97] X. Zotos, F. Naef, and P. Prelovsek. Transport and conservation laws. In: Phys. Rev. B 55 (1997), p. 11029.

[Zot99] X. Zotos. Finite Temperature Drude Weight of the One-Dimensional Spin-1/2 Heisenberg Model. In: Phys. Rev. Lett. 82 (1999), p. 1764.

[Zwi11] G. Zwicknagl. Field-induced suppression of the heavy-fermion state in $Y b R h_{2} S i_{2}$. In: J. Phys.: Condens. Matter 23 (2011), p. 094215.

[Zwi92] G. Zwicknagl. Quasi-particles in heavy fermion systems. In: Adv. Phys. 41 (1992), p. 203. 


\section{Acknowledgements}

Modern scientific work - and the present one is no exception - is mostly carried out in collaboration with many scientists. In our case this begins with the crystal growth, includes other measurements, technical support, and finalizes with the interpretation of results and theoretical calculations.

The first study of the Wiedemann-Franz law on $\mathrm{YbRh}_{2} \mathrm{Si}_{2}$ was an long-standing project initiated and supervised by Frank Steglich. The samples were grown by Cornelius Krellner in the group of Christoph Geibel. Steffen Wirth prepared the gold pads for the thermometer contacts. Stefanie Hartmann built the set-up and performed the first measurements. Additional electrical resistivity measurements were performed by Stefan Lausberg. The data analysis was supported by Ulrike Stockert, Manuel Brando, and Peijie Sun. Theoretical support came from Qimiao Si and Stefan Kirchner. Apart from the people already mentioned, valuable input for the interpretation of the data and for the later manuscript also came from Sven Friedemann and Elihu Abrahams.

The project on Kondo lattices in magnetic field was initiated by Ramzy Daou with the measurements across the metamagnetic transition in $\mathrm{CeRu}_{2} \mathrm{Si}_{2}$. He constructed the sample holder. Together, we prepared the set-up and performed the measurements on both $\mathrm{CeRu}_{2} \mathrm{Si}_{2}$ and $\mathrm{YbRh}_{2} \mathrm{Si}_{2}$. We had strong support by Manuel Brando both experimentally and concerning the interpretation of the results. The sample of $\mathrm{CeRu}_{2} \mathrm{Si}_{2}$ was grown by Stefan Tautz in Cambridge, the one of $\mathrm{YbRh}_{2} \mathrm{Si}_{2}$ was again provided by Cornelius Krellner. Additional resistivity measurements on $\mathrm{YbRh}_{2} \mathrm{Si}_{2}$ were performed by Tanja Westerkamp supervised by Philipp Gegenwart and by Stefan Lausberg. Gertrud Zwicknagl from Braunschweig performed the renormalized band-structure calculations. Apart from Ramzy Daou and Manuel Brando, the interpretation of the results was strongly influenced by the ideas from Christoph Geibel, Steffen Wirth, and Getrud Zwicknagl.

The samples for the third project on $\mathrm{LaPt}_{4} \mathrm{Ge}_{12}$ were grown by Roman Gumeniuk in the group of Andreas Leithe-Jasper, which are members of the chemistry department of Juri Grin at the MPI CPfS. Michael Nicklas supported me in the resistivity and specific heat measurements. Walter Schnelle provided valuable information about other properties of this system. The thermal-conductivity set-up was initially built by Hugo Vieyra. Ulrike Stockert was involved in the data interpretation.

I would like to thank all involved scientists for their commitment, support, and assistance as well as for fruitful discussions during these projects.

I especially want to express my sincere gratitude to Frank Steglich, who gave me the opportunity to perform advanced and challenging research in the field of strongly correlated rare-earth systems in the Max-Planck institute. I am thankful for his strong support, both in physical matters and for my future career.

I am also deeply grateful to Andy Mackenzie for his patience to let me finish my $\mathrm{PhD}$ at the MPI CPfS after the retirement of Frank Steglich as a director. I want to thank him for his support and advice during my post-doc search. 
I would like to thank Joachim Wosnitza and Cornelius Krellner for their work as referees of this thesis.

I am much indebted to Ulrike Stockert, who was my direct supervisor during my PhD. She had an open ear for my problems both physically and personally and I could always count on her support. I want to thank her for many inspiring discussions. She was a critical reader of all my documents. While Ulrike was on parental leave twice, I had the great opportunity to learn from many outstanding scientists at our institute (some called it multiple supervision).

Among these scientists, I first want to thank Ramzy Daou and Manuel Brando. At the beginning of my $\mathrm{PhD}$, there was a lack of set-ups ready to use for the first intended investigations. Therefore, I joined Ramzy's and Manuel's project as a newbie in terms of dilution refrigerators - on the oldest of all fridges in the institute. Out of this coincidence grew an inspiring and successful collaboration over the years, which we will-I am sure - continue to follow Mott's and Lifshitz's footsteps. I want to thank both of them for uncountable coffee and something-with-apple-cake afternoon discussions in the coffee corner and for their invaluable scientific and personal support.

Alexander Steppke was another regular member of these coffee sessions. We shared many of the ups and downs during our PhD time and had countless discussions about science and the rest of the life. I want to thank him for his support and many hours of fun in the lab.

I am very grateful for the help in all different matters to Christoph Geibel. After discussion with him, I most often stepped out of his office with new inspirations and a little wiser than before.

I also want to thank many other colleagues in the department. Especially, I would like to mention here my office mates and thank them for the wonderful office atmosphere. I very much enjoyed the discussions with Tobias Förster and had a lot of fun with Zita Hüsges preparing our fantastic Christmas party. I also thank her for fast proof reading the thesis. I thank Thomas Gruner and Stefan Lausberg for the exciting journey with S. Kansen; Stefanie Hartmann for many enjoyable hours with "Franz" in the lab; Steffen Wirth and Michael Nicklas for their invaluable support.

I am much obliged to our skilled technicians, who take care that the labs and cryostats stay cool.

A very special thank you goes to my parents, Stefan, Bastian, and my friends. 


\section{List of Publications}

F. Steglich, H. Pfau, S. Lausberg, S. Hamann, P. Sun, U. Stockert, M. Brando, S. Friedemann, C. Krellner, C. Geibel, S. Wirth, S. Kirchner, E. Abrahams, Q. Si, Evidence for a Kondo Destroying Quantum Critical Point, J. Phys. Soc. Jpn. 83, 061001 (2014).

D. Das, T. Gruner, H. Pfau,U. B. Paramanik, U. Burkhardt, C. Geibel, Z. Hossain, Heavy fermion and Kondo lattice behavior in the itinerant ferromagnet $\mathrm{CeCrGe}$, J. Phys.: Cond. Mat. 26, 106001 (2014).

H. Pfau, R. Daou, S. Lausberg, H. R. Naren, M. Brando, S. Friedemann, S. Wirth, T. Westerkamp, U. Stockert, P. Gegenwart, C. Krellner, C. Geibel, G. Zwicknagl, F. Steglich, Interplay between Kondo Suppression and Lifshitz Transitions in $\mathrm{YbRh}_{2} \mathrm{Si}_{2}$ at High Magnetic Fields, Phys. Rev. Lett. 110, 256403 (2013).

U. Stockert, H. Pfau, S. Hartmann, S. Lausberg, S. Friedemann, C. Krellner, S. Wirth, C. Geibel, F. Steglich, Influence of Ir and La substitution on the thermal transport properties of $\mathrm{YbRh}_{2} \mathrm{Si}_{2}$, Phys. Stat. Solidi B 250, 491 (2013).

H. Pfau, S. Hartmann, U. Stockert, P. Sun, S. Lausberg, M. Brando, S. Friedemann, C. Krellner, C. Geibel, S. Wirth, S. Kirchner, E. Abrahams, Q. Si, F. Steglich, Thermal and electrical transport across a magnetic quantum critical point, Nature 484, 493 (2012).

H. Pfau, R. Daou, M. Brando, F. Steglich, Thermoelectric transport across the metamagnetic transition of $\mathrm{CeRu}_{2} \mathrm{Si}_{2}$, Phys. Rev. B 85, 035127 (2012).

C. Krellner, S. Lausberg, A. Steppke, M. Brando, L. Pedrero, H. Pfau, S. Tencé, H. Rosner, F. Steglich, C. Geibel, Ferromagnetic quantum criticality in the quasi-one-dimensional heavy fermion metal $\mathrm{YbNi}_{4} \mathrm{P}_{2}$, New Journal of Physics 13, 103014 (2011). 



\section{Versicherung}

Hiermit versichere ich, dass ich die vorliegende Arbeit ohne unzulässige Hilfe Dritter und ohne Benutzung anderer als der angegebenen Hilfsmittel angefertigt habe. Die aus fremden Quellen direkt oder indirekt übernommenen Gedanken sind als solche kenntlich gemacht. Die Arbeit wurde bisher weder im Inland noch im Ausland in gleicher oder ähnlicher Form einer anderen Prüfungsbehörde vorgelegt.

Die vorliegende Doktorarbeit wurde am Max-Planck-Institut für Chemische Physik fester Stoffe in Dresden unter der wissenschaftlichen Betreuung von Herrn Prof. Dr. Frank Steglich und Frau Dr. Ulrike Stockert angefertigt.

Ich erkenne die Promotionsordnung der Technischen Universität Dresden an.

Dresden, den 16.12.2014

Heike Pfau 